\title{
ANÁLISE ESTRUTURAL DAS CHAPAS METÁLICAS DE SILOS E DE RESERVATÓRIOS CILÍNDRICOS
}

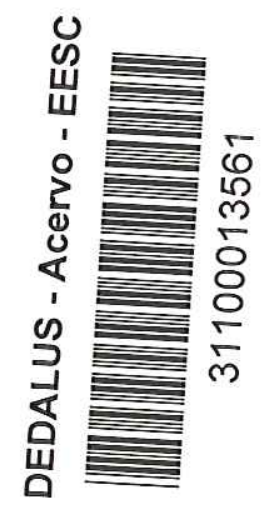

\author{
Luciano Jorge de Andrade Junior
}

Dissertação apresentada à Escola de Engenharia de São Carlos da Universidade de São Paulo, como parte dos requisitos para obtenção do título de Mestre em Engenharia de Estruturas

ORIENTADOR: Prof. Dr. José Jairo de Sáles

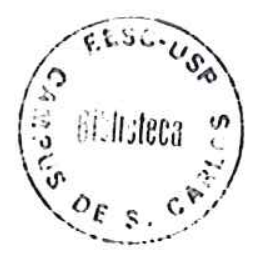

São Carlos

1998 
Candidato: Engenheiro LUCIANO JORGE DE ANDRADE JUNIOR

Dissertação defendida e aprovada em 19-05-1998 pela Comissão Julgadora:

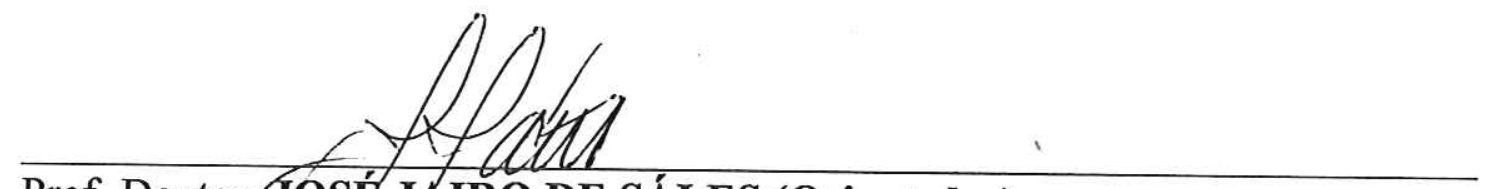

Prof. Doutor JOSÉ JAIRO DE SÁLES (Orientador)

(Escola de Engenharia de São Carlos - Universidade de São Paulo)

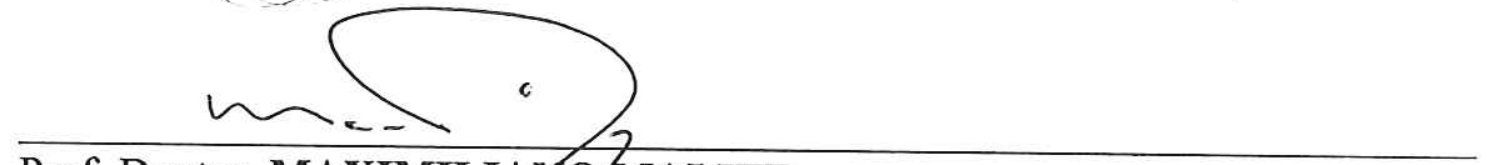

Prof. Doutor MAXIMILIANO MIALITE

(Escola de Engenharia de\$são Carlos - Universidade de São Paulo)

Prof. Doutor JULIO FRUCHTENGARTEN

(Escola Politécnica - Universidade de São Paulo)

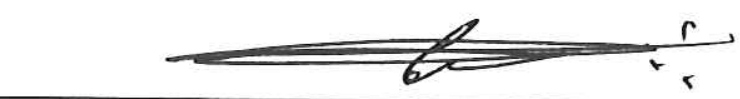

Prof. Titular CARLITO CALIL JUNIOR

Coordenador da Área de Engenharia de Estruturas

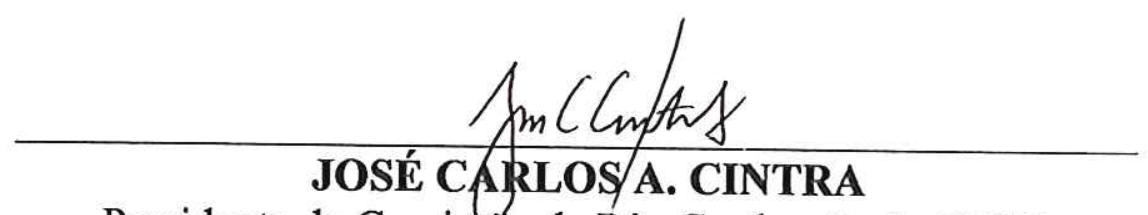

Presidente da Comissáo de Pós-Graduação da EESC 
Ao meu pai,

orientador natural. 
Ao sempre disposto professor José Jairo de Sáles, minha estima pela orientação neste trabalho e minha admiração pela sua maneira clara e direta de se expressar.

Ao Conselho Nacional de Pesquisa - CNPq, pela bolsa de estudo concedida.

Aos amigos Arthur Dias, companheiro distinto em momentos de luta, Luciano Barbosa, um ponderador, e Júlio Pituba, um sujeito seguro, meu profundo respeito e um agradecimento largo pela sempre participação em minha vida na cidade de São Carlos.

Aos colegas, professores e funcionários do Departamento de Estruturas, sempre no dia-a-dia das aulas, nos momentos do café, meus sinceros agradecimentos pela prosa, pelo muito aprendido e pela colaboração em minhas atividades de mestrado. 


\section{SUMÁRIO}

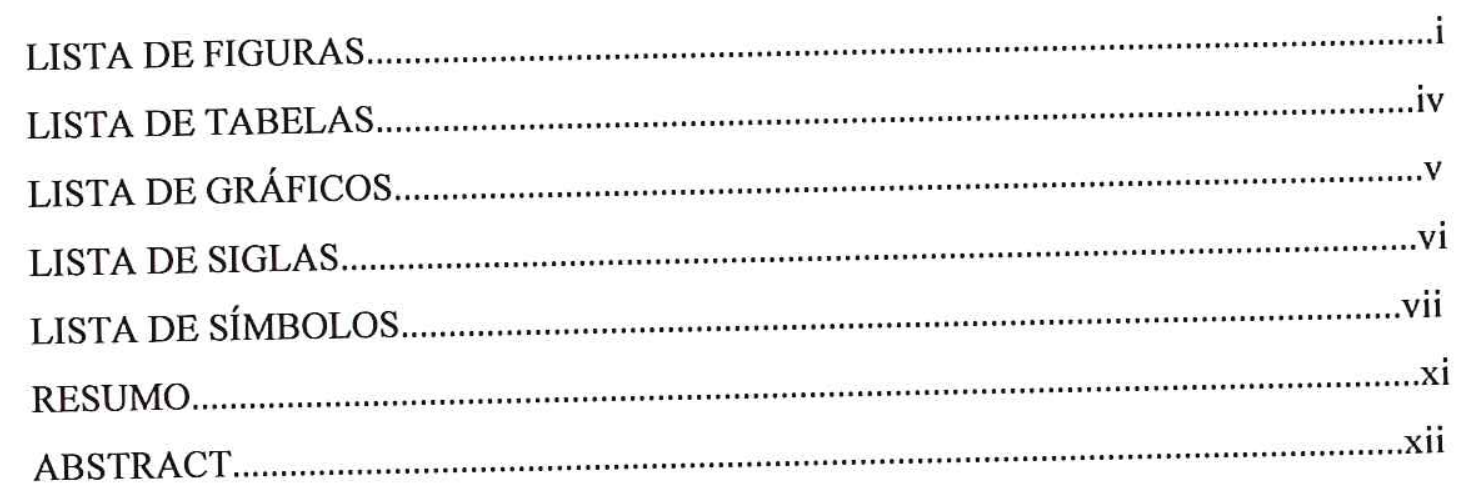

CAPÍTULO 1 - Ambiente onde se desenvolve a Dissertação............................................... 1

1.1 - Introdução. 1

1.2 - Visão geral do corpo da Dissertação.

.. 6

CAPÍTULO 2: Comportamento Estrutural e Ações de Silos e de Reservatórios. 7

2.1 - Introdução....................................

2.2 - Esforços Solicitantes e Equações de Equilíbrio............................................................. 9

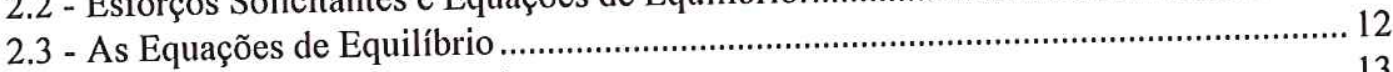

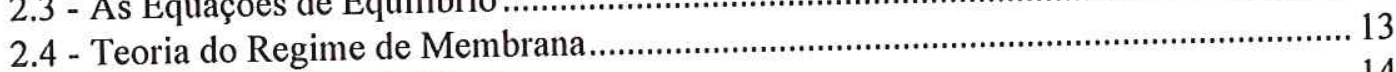

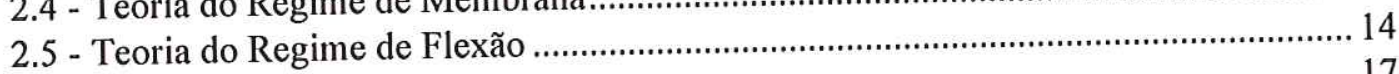

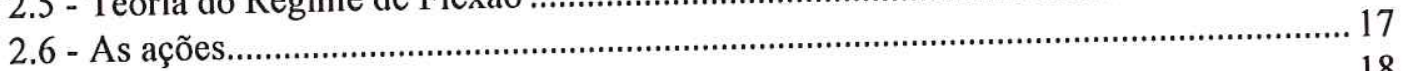

2.6.1 - A Ação do Vento ................................................................................................ 18

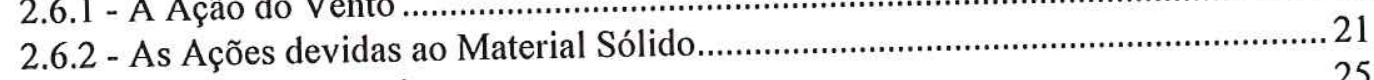

2.6.3 - A Ação Hidrostática. ........................................................................................... 25

CAPÍTULO 3 - Análise do problema de estabilidade em cascas cilíndricas....................26

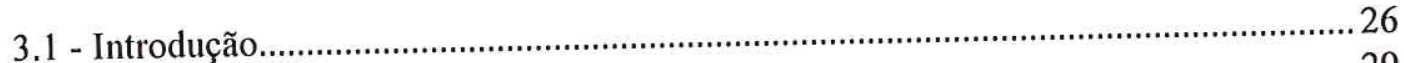

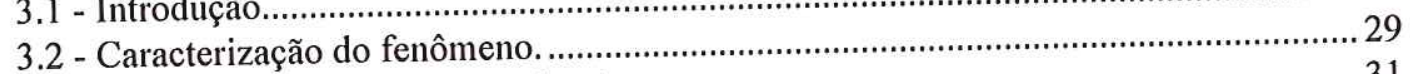

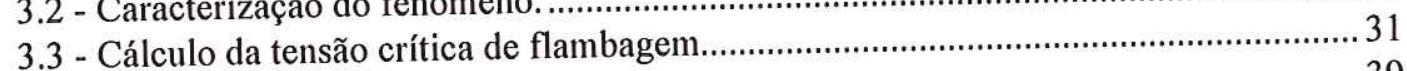

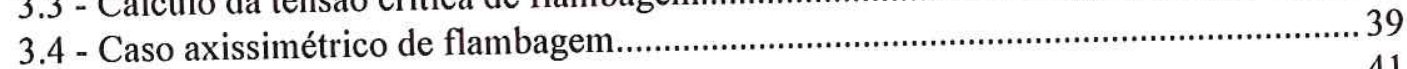

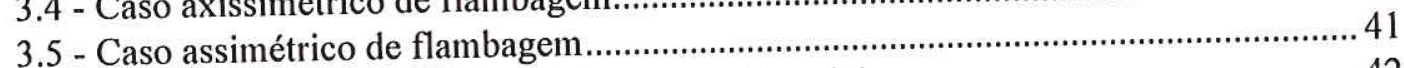

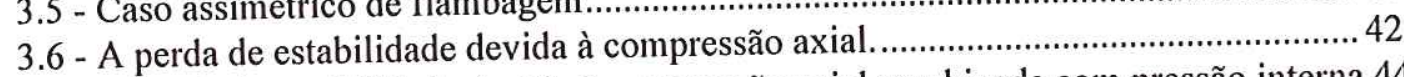

3.7 - A perda de estabilidade devida à compressão axial combinada com pressão interna.44

3.8 - A perda de estabilidade devida à compressão axial combinada com flexão................ 45 
CAPÍTULO 4 : Análise Numérica de Cascas Cilíndricas...................................................... 47

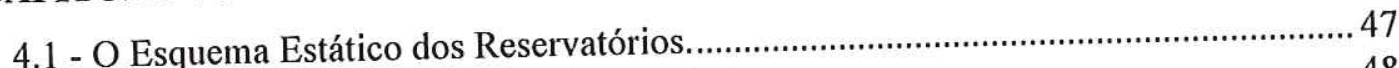

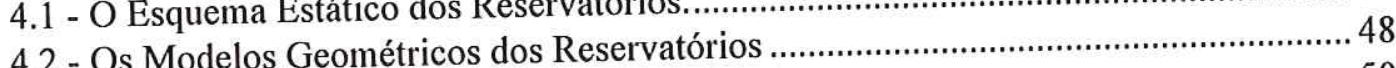

4.3 - As Características do Aço Empregado.......................................................................... 50

4.4 - Considerações acerca das Espessuras das Chapas. ......................................................... 50

4.5 - Os Casos em Análise $\ldots$

53

53

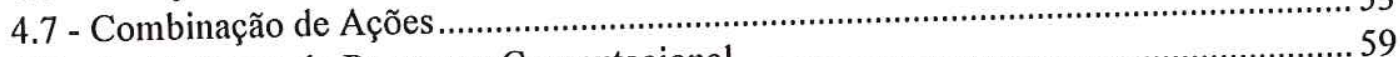

4.8 - As hipóteses do Programa Computacional.............................................................. 61

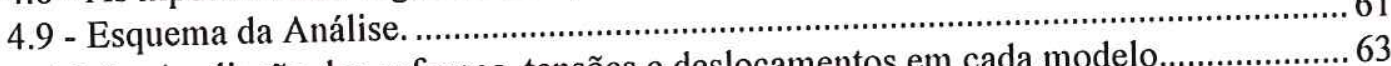

4.9.1 - Avaliação dos esforços, tensões e deslocamentos em cada modelo...................... 63

4.9.2 - Comparação das regiões de tensão entre os modelos.

CAPíTULO 5 - Caso Exemplo: Silo de Grãos

CAPÍTULO 6: Conclusões 


\section{LISTA DE FIGURAS}

Figura $1 \diamond$ - Tanque de álcool de $10.000 \mathrm{~m}^{3}$

1

Figura $2 \diamond$ - Silo de açúcar.

1

Figura 3 - Reservatório de água.

2

Figura $4 \diamond$ - Estruturas cilíndricas em fase de montagem......................................................... 3

Figura 5 - Afundamento do topo de silo sob a ação do vento................................................... 3

Figura 6 - Amassamento do costado do silo devidoà ação do vento.......................................... 3

Figura 7 - Ações axissimétricas: seção eficiente..................................................................... 3

Figura 8 - Tensões meridionais: podem provocar flambagem................................................. 4

Figura 9 - A resistência à flexão é menor que a resistência à compressão ................................ 5

Figura 10 - Partes da Estrutura: Tampa, corpo e fundo ........................................................... 7

Figura 11 - Esforços Solicitantes Generalizados.................................................................. 9

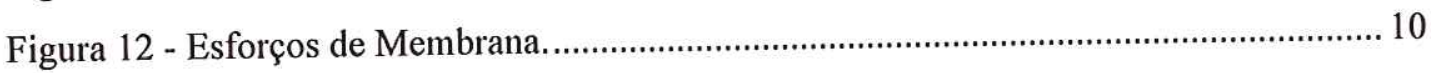

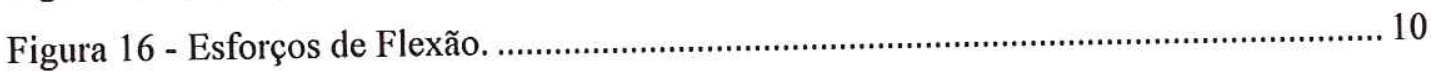

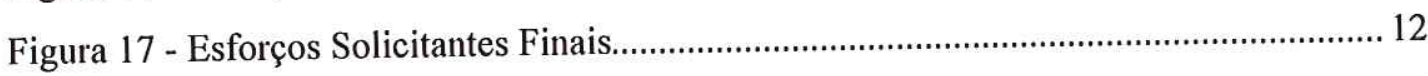

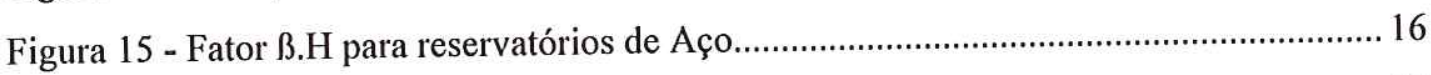

Figura 16 - Fator ß.H para reservatórios de Concreto........................................................... 17

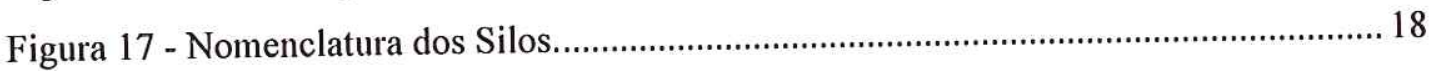

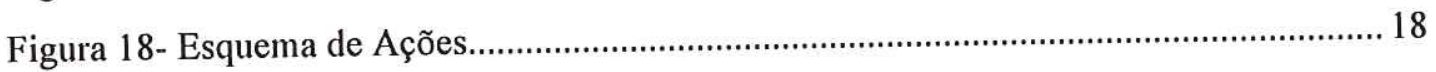

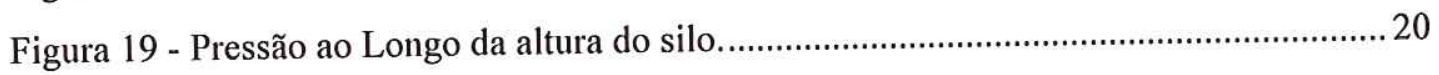

Figura 20 - Configuração dos coeficientes $\mathrm{C}_{\text {pe }}$ no perímetro do silo......................................2 20

Figura 21 - Distribuição dos Cpe em cobertura plana.............................................................. 20

Figura 22 - Distribuição dos $\mathrm{C}_{\mathrm{pe}}$ em cobertura cônica............................................................ 20

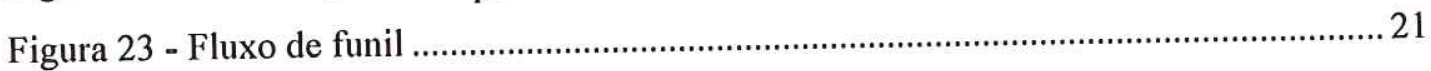

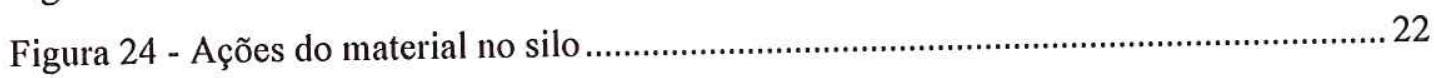

Figura 25 - Configuração de flambagem elástica de cilindro comprimido...............................2 26 
Figura 26 - Modo Geral de Flambagem periódica na circunferência de um silindro longo.. 27

Figura 27 - Modo axissimétrico de flambagem local.

Figura 28 -Disposição das chapas calandradas e regiões de tensões residuais.........................22

Figura 29 -Caminhos fundamental e secundário de flambagem.............................................. 30

Figura 30 -Estado fundamental de deformação..................................................................... 32

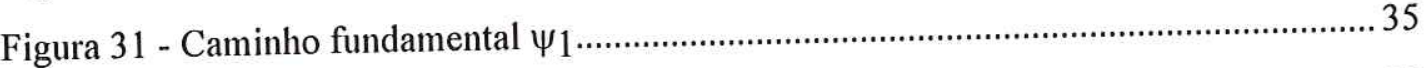

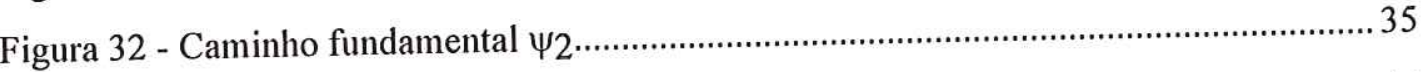

Figura 33 - Medidas de Imperfeições................................................................................ 44

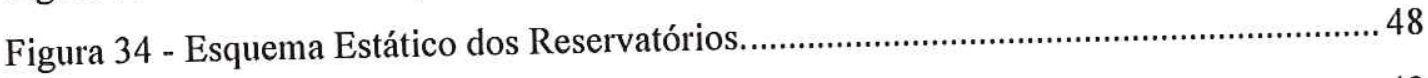

Figura 35 - Modelos Geométricos de Reservatórios Cilíndricos............................................ 49

Figura 36 - Variaçãodas Chapas ao Longo da Altura............................................................. 50

Figura 37 - Elemento sob Força Tangencial .............................................................................5

Figura 38 - Caso 1 - Sem Atrito: Parede de Espessura Constante ……....................................5 51

Figura 39 - Caso 2 - Sem Atrito: Parede de Espessura Variável................................................ 51

Figura 40 - Caso 3 - Com Atrito: Parede de Espessura Constante............................................. 52

Figura 41 - Caso 4 - Com Atrito: Parede de Espessura Variável............................................... 52

Figura 42 - Caso 5 - Reservatório Vazio com Parede de Espessura Constante........................ 52

Figura 43 - Caso 6 - Reservatório Vazio com Parede de Espessura Variável ..........................5 53

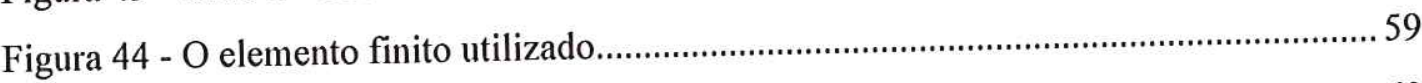

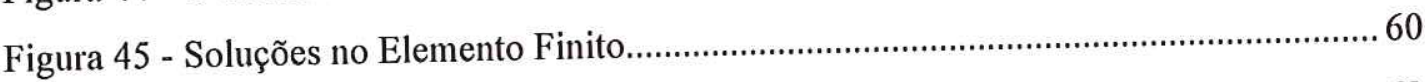

Figura 46 - Malhas e rede de elementos finitos nos modelos.................................................62 62

Figura 47 - Espessuras do costado para o modelo 1 - Casos 1 e 2.............................................6 65

Figura 48 - Espessuras do costado para o modelo 1 - Casos 3 e 4 .............................................6 69

Figura 49 - Tensões tangenciais $\sigma_{\mathrm{y}}$ - Modelo 1 - Caso 5 …………………….......................... 74

Figura 50 - Tensões tangenciais $\sigma_{\mathrm{y}}$ - Modelo 1 - Caso 6 .......................................................... 74

Figura 51 - Tensões meridionais $\sigma_{\mathrm{Z}}$ - Modelo 1 - Caso 5 .......................................................... 75

Figura 52 - Tensões meridionais $\sigma_{\mathrm{Z}}$ - Modelo 1 - Caso 6........................................................ 75

Figura 53 - Espessuras do costado para o modelo 5 - Casos 1 e 2 2............................................ 77

Figura 54 - Espessuras do costado para o modelo 5 - Casos 3 e 4 ............................................. 81

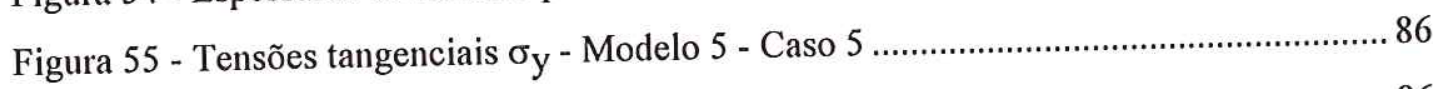

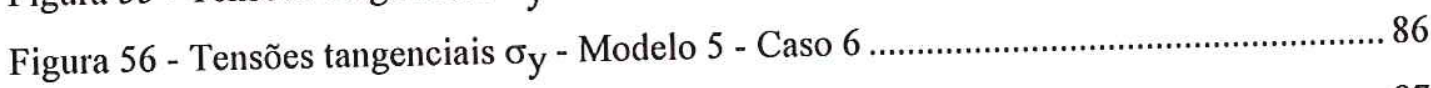

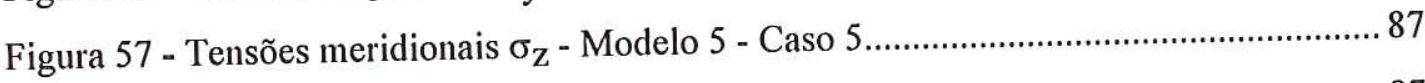

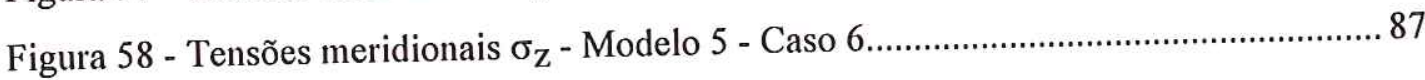




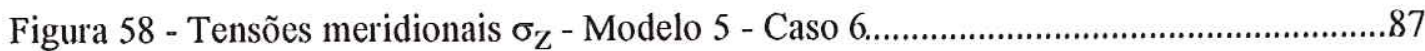

Figura 59 - Faixas de Coeficientes de Pressão em cobertura cônica.......................................91

Figura 60 - Valores das pressões na cobertura do silo exemplo..............................................92

Figura 61 - Áreas das faixas de pressão.............................................................................9.

Figura 62 - Esforços transmitidos pela tampa ao costado do silo...........................................93

Figura 63 - Esquema da cobertura cônica..............................................................................26

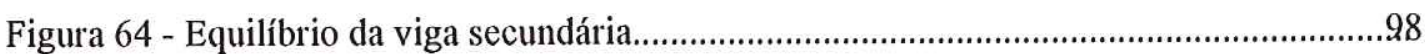

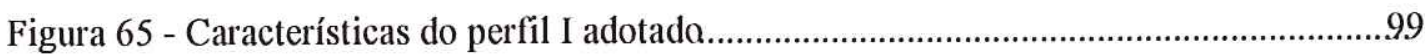

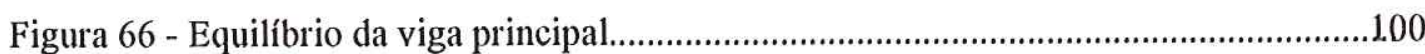

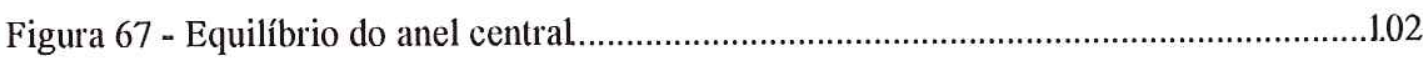

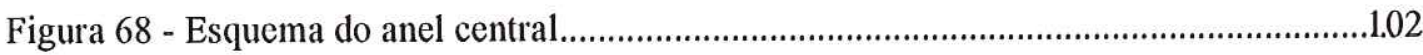

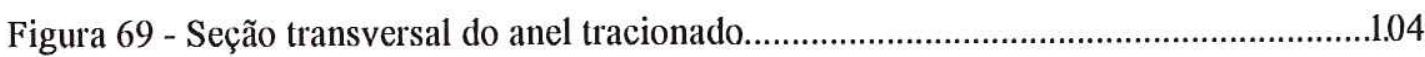

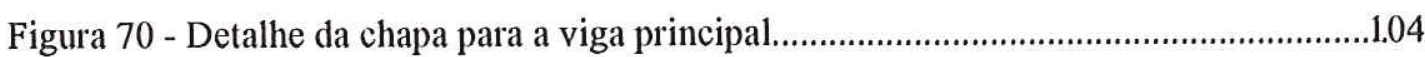

Figura 71 - Detalhe da ligação da viga de topo à viga secundária........................................104

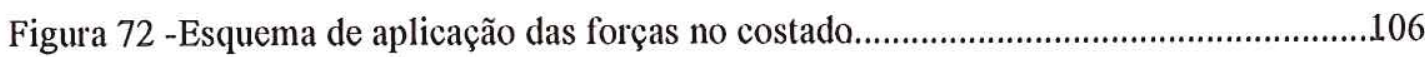

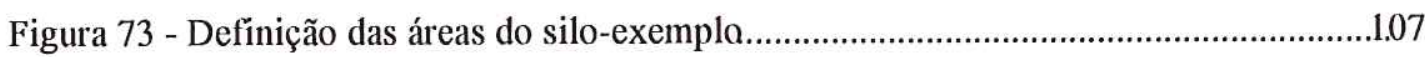

Figura 74 - Mapa dos elementos finitos na casca do silo exemplo........................................108

Figura 75 - Mapa dos nós dos elementos finitos na casca do silo exemplo...........................109

Figura 76 - Tensões meridionais $\sigma_{\mathrm{Z}}$ - solicitação: vento - parte interna da casca..................112

Figura 77 - Tensões meridionais $\sigma_{Z}$ - solicitação: vento - superfície média..........................112

Figura 78 - Tensões meridionais $\sigma_{\mathrm{Z}}$ - solicitação: vento - face externa da casca...................1.13

Figura 79 - Tensões meridionais $\sigma_{\mathrm{Z}}$ - solicitação: grãos - face interna da casca..................113

Figura 80 - Tensões meridionais $\sigma_{\mathrm{Z}}$ - solicitação: grãos - superfície média...........................114

Figura 81 - Tensões meridionais $\sigma_{\mathrm{Z}}$ - solicitação: grãos - face externa da casca..................114

Figura 82 - Espessuras do costado do silo exemplo...............................................................1.15

Figura 83 - Regiões de concentração de tensões nos modelos 1 e 5......................................117

$\diamond$ As fotos corresopndentes às figuras 1, $2 \mathrm{e} 4$ foram feitas pelo Renato Celine Badiale. 


\section{LISTA DE TABELAS}

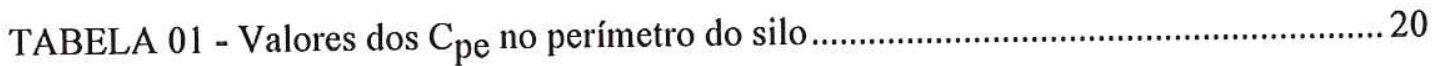

TABELA 02 - Relações altura/diâmetro e área lateral para um volume constante..................49

TABELA 03 -Pressões devidas ao material - Modelo 1 .......................................................... 55

TABELA 04 - Pressões do vento ao longo da altura e do perímetro - Modelo 1................... 55

TABELA 05 - Pressões do vento ao longo da altura e do perímetro - Modelo 5.................. 56

TABELA 06 - Pressões devidas ao material armazenado - Modelo 5...................................... 56

TABELA 07 - Valores para as forças de atrito por unidade de comprimento da geratriz em

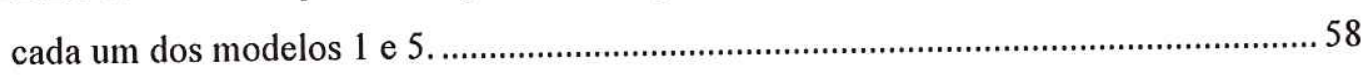

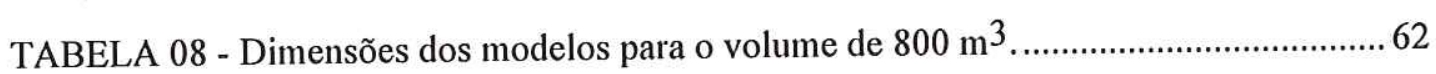

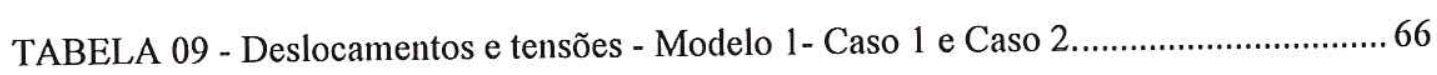

TABELA 10 - Deslocamentos e tensões - Modelo 1-Caso 3 e Caso 4 ……...............................70

TABELA 11 - Deslocamentos e tensões - Modelo 5 - Caso 1 e Caso 2..................................... 78

TABELA 12 - Deslocamentos e tensões - Modelo 5 - Caso 3 e Caso 4 …................................ 82

TABELA 13 - Pressões do vento ao longo da altura e do perímetro........................................94

TABELA 14 - Variações de espessuras testadas para o silo exemplo.................................... 110

TABELA 15 - Verificação à flambagem para flexão e compressão........................................ 110

TABELA 16 - Verificação à flambagem para compressão e pressão interna......................... 111 


\section{LISTA DE GRÁFICOS}

Gráfico 1 - Deslocamentos Radiais - Modelo 1- Casos 1 e 2 …...............................................6 67

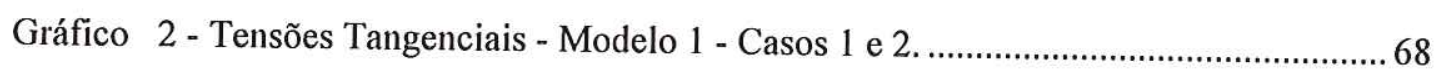

Gráfico 3 - Deslocamentos Radiais - Modelo 1 - Casos 3 e 4 ................................................ 71

Gráfico 4 - Tensões Tangenciais - Modelo 1 - Casos 3 e 4 ................................................... 72

Gráfico 5 - Tensões Meridionais - Modelo 1 - Casos 3 e 4 ..................................................... 73

Gráfico 6 - Deslocamentos Radiais - Modelo 5- Casos 1 e 2 ................................................. 79

Gráfico 7 - Tensões Tangenciais - Modelo 5 - Casos 1 e 2 ..................................................... 80

Gráfico 8 - Deslocamentos Radiais - Modelo 5 - Casos 3 e 4 ................................................ 83

Gráfico 9 - Tensões Tangenciais - Modelo 5 - Casos 3 e 4 .................................................. 84

Gráfico 10 - Tensões Meridionais - Modelo 5 - Casos 3 e 4 …….......................................... 85 


\section{LISTA DE SIGLAS}

$\begin{array}{ll}\text { AISC } & \text { - American Institute of Steel Construction } \\ \text { ASCE } & \text { - American Society of Civil Engineers } \\ \text { DIN } & \text { - Deutsche Industrie Normen } \\ \text { ECCS } & \text { - European Convention for Constructional Steelwork } \\ \text { NBR } & \text { - Norma Brasileira Registrada }\end{array}$




\section{LISTA DE SÍMBOLOS}

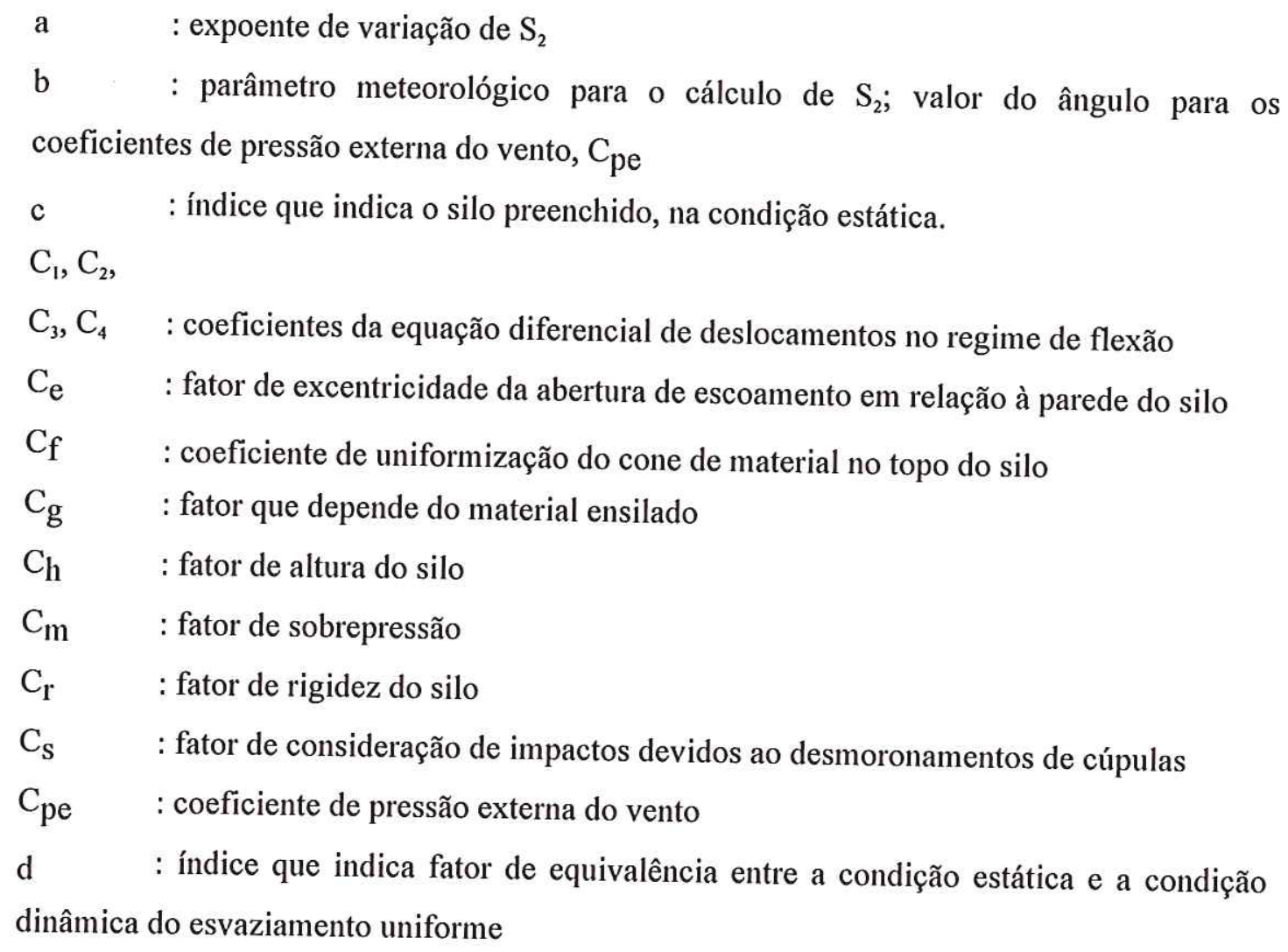


$E_{N}^{z} \quad$ : termo de contribuição axial (direção da geratriz) da energia de deformação linear de membrana

$E_{N}^{\theta} \quad$ : termo de contribuição circunferencial (ou tangencial) da energia de deformação linear de membrana

$E_{N}^{z \theta} \quad$ : termo de contribuição devida ao cisalhamento, da energia de deformação linear de membrana

$\mathrm{E}_{\mathrm{pt}} \quad$ : energia potencial total

$\mathrm{E}_{\mathrm{pt} 0,} \mathrm{E}_{\mathrm{pt} 1}$,

$\mathrm{E}_{\mathrm{pt} 2} \quad$ : termos da energia potencial total

$\mathrm{F}_{\mathrm{r}} \quad$ : fator de rajada do vento

fy : tensão limite de escoamento

$F_{X}, F_{x y}$,

Fy : esforços de membrana no elemento finito do ANSYS (Shell 63)

$\mathrm{H} \quad$ : altura do silo

: número de semi-ondas formadas na casca cilíndrica, na direção circunferencial,

na configuração prevista de flambagem

iCM : número crítico mínimo de semi-ondas na direção circunferencial

j

: número de semi-ondas formadas na direção da geratriz, na configuração prevista

de flambagem

k : distância entre o eixo vertical da abertura de escoamento e a parede do silo

$\mathrm{K}_{\mathrm{d}} \quad$ : fator dinâmico considerado quando da descarga do silo

$l_{\mathrm{r}} \quad$ : comprimento relacionado ao tamanho potencial das ondas de flambagem; pode

ser medido tanto na direção da geratriz quanto na direção circunferencial

$\mathrm{l}_{\mathrm{Z}} \quad$ : comprimento da semi-onda na direção da geratriz

lo : comprimento da semi-onda na circunferência

$\mathrm{M}_{\mathrm{X}}, \mathrm{M}_{\mathrm{y}}$,

$\mathrm{M}_{\mathrm{Xy}} \quad$ : momentos por unidade de comprimento, no elemento finito do ANSYS (Shell

63)

p (P) : pressão horizontal atuando na parede do silo; o índice que estiver subscrito a $P$ indica a condição de solicitação (estático (Janssen), dinâmico (esvaziamento))

$\mathrm{P}_{\mathrm{cr}} \quad$ : valor de solicitação crítica

$q(Q)$ : pressão vertical atuando na direção do eixo do silo; o índice que estiver subscrito a q ou Q indica a condição de solicitação ( estático (Janssen), dinâmico (esvaziamento)) 


$$
\begin{array}{ll}
\text { qf } & \text { : pressão vertical total (do material ensilado) resultante no fundo do silo } \\
\mathrm{R} & \text { : raio hidráulico da estrutura cilíndrica } \\
\mathrm{S} & \text { : área da seção transversal de massa ensilada } \\
\mathrm{S}_{1} & \text { : fator topográfico, usado na avaliação das variações do relevo do terreno } \\
\mathrm{S}_{2} & \text { : fator para a consideração do "efeito combinado da rugosidade do terreno, da } \\
\text { variação da velocidade do vento com a altura acima do terreno e das dimensões da } \\
\text { edificação." (NBR } 6123 \text { (1987)) }
\end{array}
$$

$\mathrm{S}_{3} \quad$ : fator estatístico usado para a avaliação do grau de segurança e da vida útil da estrutura

$\bar{u} \quad$ : medida de imperfeição a partir da imperfeição até a direção de $\ell_{\mathrm{r}}$

$\mathrm{U} \quad$ : perímetro da seção $\mathrm{S}$

$\mathrm{u}, \mathrm{v}, \mathrm{w} \quad$ : deslocamentos correspondentes às coordenadas $\mathrm{x}, \mathrm{y}, \mathrm{z}$

V : volume de um silo cilíndrico

$\mathrm{V}_{\mathrm{k}} \quad$ : velocidade característica do vento

$\mathrm{V}_{\mathrm{cs}} \quad$ : resultante da força de atrito, em unidade de força por unidade de comprimento

v : : força de atrito na parede por unidade de área

$\mathrm{v}_{1} \quad$ : deslocamento fundamental na direção radial do silo

$\mathrm{x}, \mathrm{y}, \mathrm{z} \quad$ : coordenadas cartesianas

$\mathrm{w}_{1}$ : deslocamento fundamental na direção da geratriz do silo

wh : solução homogênea da equação diferencial dos deslocamentos w

$w_{\mathrm{p}} \quad$ : solução particular da equação diferencial dos deslocamentos w

$\mathrm{r}, \theta, \mathrm{z} \quad$ : coordenadas cilíndricas

z : coordenada na direção da geratriz do cilindro; também indica altura medida a partir da superfície do terreno

$\alpha_{\mathrm{Z}} \quad$ : constante de ponderação de $\mathrm{N}_{\mathrm{Zf}}$ ( vale 1, na condição de solicitação predominante na direção da geratriz

$\alpha_{\theta} \quad$ : constante de ponderação de $\mathrm{N}_{\theta \mathrm{f}}$ ( vale 1 , na condição de pressão externa dominante)

$\beta \quad$ : coeficiente de amortecimento dos esforços na casca cilíndrica; ou, razão entre a tensão meridional de membrana e a tensão efetiva de membrana, até o início de flambagem

$\Delta \mathrm{P} \quad$ : componente estática de pressão do vento

$\varepsilon \quad$ : deformação na direção tangencial, $\theta$

$\varepsilon_{\mathrm{Zf}}:$ deformação uniforme fundamental, na direção da geratriz, associada a $\mathrm{N}_{\mathrm{Zf}}$ 
$\varepsilon_{\theta f} \quad:$ deformação uniforme fundamental, na direção circunferencial, associada a $\mathrm{N}_{\mathrm{Zf}}$

$\phi \quad$ : ângulo de atrito entre as partículas do material ensilado (ângulo de atrito interno)

$\phi \quad$ : ângulo de atrito entre o material ensilado e o costado do silo

$\phi(\mathrm{z}) \quad$ : função exponencial do comportamento da pressão lateral do material ensilado

$\gamma \quad$ : densidade do material armazenado

$\lambda=\mathrm{p} / \mathrm{q} \quad$ : razão entre as pressões horizontal e vertical

$\mu \quad$ : coeficiente de atrito entre as partículas do material ensilado(coeficiente de atrito interno.)

$\mu^{\prime} \quad$ : coeficiente de atrito entre o material ensilado e as paredes da célula

$\checkmark \quad$ : coeficiente de Poisson

$\psi_{1} \quad$ : caminho fundamental de deslocamentos $\mathrm{v}$ e w de flambagem

$\quad \psi_{2}$ : caminho secundário de deslocamentos v e w de flambagem

$\sigma_{\theta}, \sigma_{\mathrm{Z}}$ : tensões nas direções tangencial e meridional, respectivamente

$\sigma_{\mathrm{cr}} \quad$ : tensão crítica de flambagem na direção da geratriz do silo

$\sigma_{\text {ef,u }} \quad$ : tensão última, de resistência, da casca cilíndrica

$\sigma_{0} \quad$ : tensão uniforme de compressão devida à força axial (direção da geratriz) de projeto

$\sigma_{\mathrm{b}} \quad$ : tensão máxima de compressão devida ao momento de projeto

$\tau_{\theta \mathrm{z}}, \tau_{\mathrm{z} \theta}$

$\tau_{\theta y}, \tau_{\mathrm{y} \theta}$

$\tau_{\mathrm{zy}}, \tau_{\mathrm{yz}}:$ tensões de cisalhamento.

$\mathrm{N}_{\mathrm{Zf}} \quad$ : esforço solicitante axial correspondente a um estado fundamental de solicitação uniforme de membrana

$\mathrm{N}_{\theta \mathrm{f}} \quad$ : esforço solicitante tangencial correspondente a um estado fundamental de solicitação uniforme de membrana

$\mathrm{N}_{\mathrm{Z}}$ : esforço interno meridional por unidade de comprimento

$\mathrm{N}_{\theta} \quad$ : esforço interno tangencial por unidade de comprimento

$\mathrm{N}_{\theta \mathrm{Z}}, \mathrm{N}_{\mathrm{Z} \theta}$ : esforços de membrana

$\mathrm{M}_{\theta} \quad$ : esforço de flexão na direção tangencial

$\mathrm{M}_{\mathrm{Z}} \quad$ : esforço de flexão na direção meridional

$\mathrm{P}_{\mathrm{y}}, \mathrm{P}_{\mathrm{z}} \quad$ : ações externas que atuam nas direções y e $\mathrm{z}$ do elemento de casca

$\mathrm{Q}_{\theta}, \mathrm{Q}_{\mathrm{Z}}$ : esforço interno cortante 


\section{RESUMO}

ANDRADE Jr., L. J. de (1998). Análise Estrutural das Chapas Metálicas de Silos e de Reservatórios Cilíndricos. São Carlos. Dissertação (Mestrado) - Escola de Engenharia de São Carlos, Universidade de São Paulo.

A aplicação de conceitos de estabilidade surge espontaneamente em estruturas metálicas e, mais destacadamente, nas estruturas de silos e de reservatórios. A parede cilíndrica que compõe o corpo desse tipo de estrutura é formada de chapas metálicas delgadas, característica que surge da grande eficiência da forma cilíndrica. Neste trabalho, procura-se dispor ao engenheiro conceitos claros e distintos do comportamento e dos fenômenos de perda de estabilidade do equilíbrio de reservatórios e de silos metálicos. São expostas as teorias para a análise de silos para materiais granulares e pulverulentos nãocoesivos e reservatórios para líquidos que não produzem gases. Mostra-se que a ação do material ensilado provoca esforços de compressão que possibilitam a ocorrência de fenômenos de perda da estabilidade da estrutura, conhecidos como flambagem no jargão técnico. Também a ação do vento pode ocasionar perda de estabilidade na estrutura vazia. Apresenta-se um resumo das teorias envolvidas, um ensaio numérico de modelos cilíndricos, um exemplo de silo de grãos, e uma compilação das normas e artigos mais atuais e abrangentes do problema de estabilidade em estruturas cilíndricas. Por fim, são estabelecidas as configurações estruturais que apresentam maiores riscos para a flambagem, e fornecidas as relações de diâmetro/espessura e de diâmetro/altura em que é possível se evitar os problemas advindos da perda da estabilidade do equilíbrio.

Palavras-chave: silos metálicos; flambagem; estruturas cilíndricas. 


\section{ABSTRACT}

ANDRADE Jr., L. J. de (1998). Structural Analysis of the Steel Plates on Silos and Tanks. São Carlos. Dissertação (Mestrado) - Escola de Engenharia de São Carlos, Universidade de São Paulo.

The application of the stability concepts appears spontaneously on steel structures and, more distinctly, on silos and tanks structures. The cylindrical wall that makes the body of this kind of structure is constituted of thin steel plates, characteristic that comes out of the great efficiency of the cylindrical shape. In this work, it is intended for the engineer clear and distinct notions about the behaviour and the loss of stability equilibrium phenomena of steel silos and tanks. The theories for the analysis of silos for non-cohesive, pulverulent and grain materials, and of tanks to liquids that produces no gases, are exposed. It is shown that the action of the bulk stored material causes compression that makes possiblethe occurrence of the phenomena of loss of the stability of the structure, commonly known in the technician jargon as buckling. Also the wind load can lead to the loss of stability of the empty structure. A summary is shown for of the involved theories, a numerical experiment with cylindrical models, an example of grain silo, and a compilation of the recommendations, norms and articles on the problem of the cylindrical structures stability. At least, it is established the structural configurations that poses major risks to buckling, and it is supplied relations of diametre/thickness and of diametre/height with whom it is possible to avoid the problems that come from the loss of the stability of equilibrium.

Keywords: steel silos; buckling; cylindrical shell structures. 


\section{Capítulo 1 - Ambiente onde se desenvolve a Dissertação.}

\section{1 - Introdução}

Os ambientes onde estão situados os silos e os reservatórios podem variar desde um campo agrícola, passando a instalações industriais, estações de distribuição de água, até refinarias e usinas de álcool. As estruturas chamadas silos armazenam grãos, farinhas e material sólido a granel, e os reservatórios armazenam água, álcool e líquidos em geral.

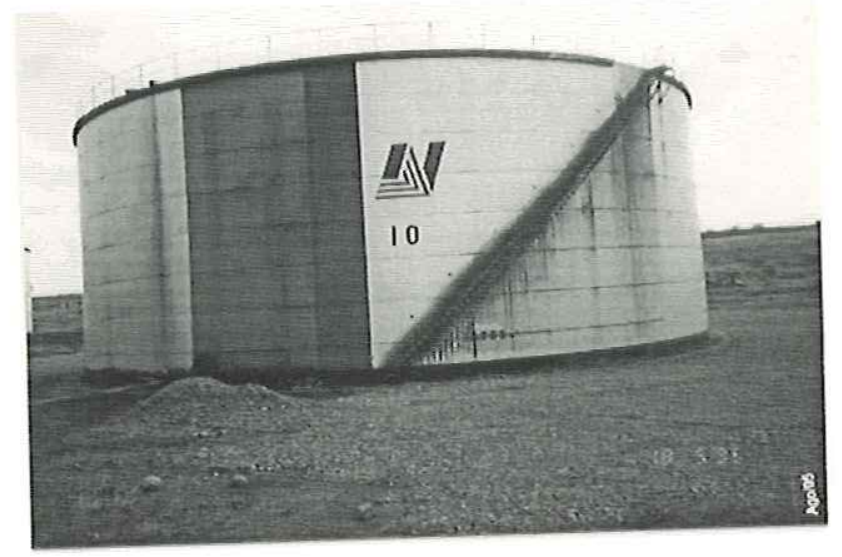

Figura 1 - Tanque de álcool de $10.000 \mathrm{~m}^{3}$

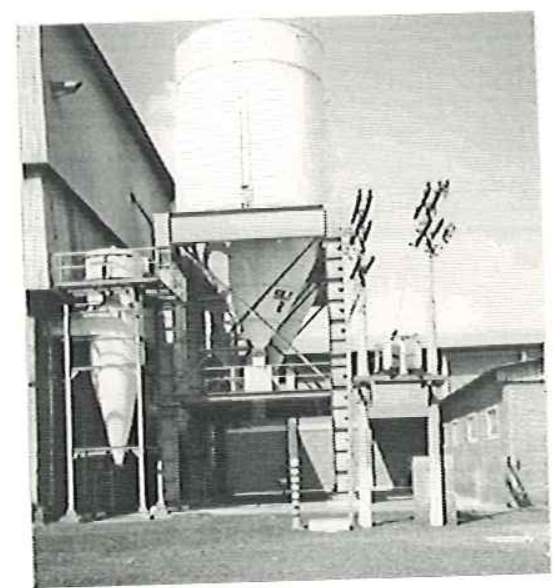

Figura 2 - Silo de açúcar. 
A figura 1 ilustra um tanque metálico de álcool, típico de regiões de cana-de-açúcar e instalações industriais de usinas; este, em particular, localizado em Assis/SP.

A figura 2 representa um silo metálico de açúcar, situado em Guaíra/SP.

A figura 3 mostra um reservatório metálico de água, de relação altura/diâmetro aproximadamente igual a 7, situado no Campus da Escola de Engenharia de São Carlos:

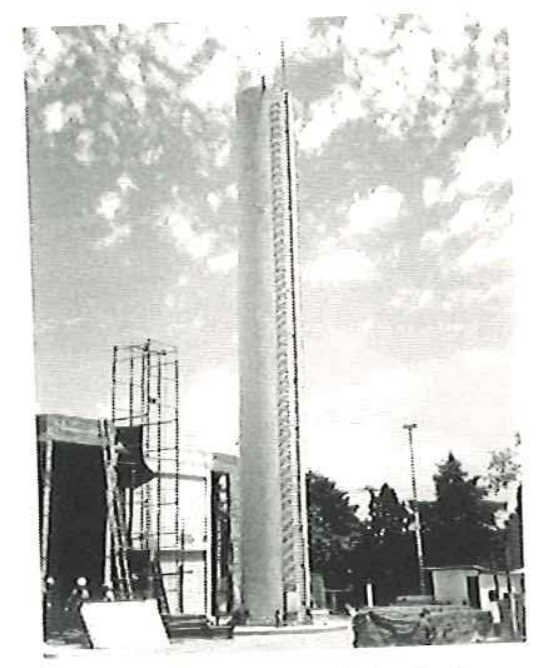

Figura 3 - Reservatório de água.

O objetivo desta dissertação é o estudo do comportamento estrutural de silos e de reservatórios quando submetidos às ações hidrostática, do vento e de material granular ou pulverulento, e da instabilidade do equilíbrio da casca cilíndrica quando submetida a esforços de compressão ao longo da geratriz.

Com este objetivo, pretende-se fornecer ao projetista o conhecimento necessário à identificação dos esforços solicitantes de membrana e de flexão, e das características do fenômeno de instabilidade do equilíbrio de cascas cilíndricas, comumente chamado flambagem. Esse conhecimento deve proporcionar condições para que sejam previstas as situações onde podem ocorrer a flambagem da estrutura.

Os esforços solicitantes devidos à ação do vento são analisados para as estruturas de silos e de reservatórios quando estão vazias, uma vez que, quando cheias, ou parcialmente cheias, a quantidade de material contida nelas é suficiente para conferir à estrutura maior estabilidade à ação do vento. Já os esforços de atrito na parede da estrutura, devidos ao material ensilado, são analisados quando o material está em repouso ou durante o esvaziamento do silo.

Uma fase à qual a estrutura cilíndrica fica bastante suscetível à ação do vento é quando da montagem do silo e ainda não está fixada a tampa (figuras 4 e 5). 
A figura 4 ilustra o caso de estruturas cilíndricas em fase de montagem. São dornas de fermentação do caldo da cana-de-açúcar, com dimensões de $12 \mathrm{~m}$ de diâmetro e $12 \mathrm{~m}$ de altura $\left(\sim 1.350 \mathrm{~m}^{3}\right)$ :

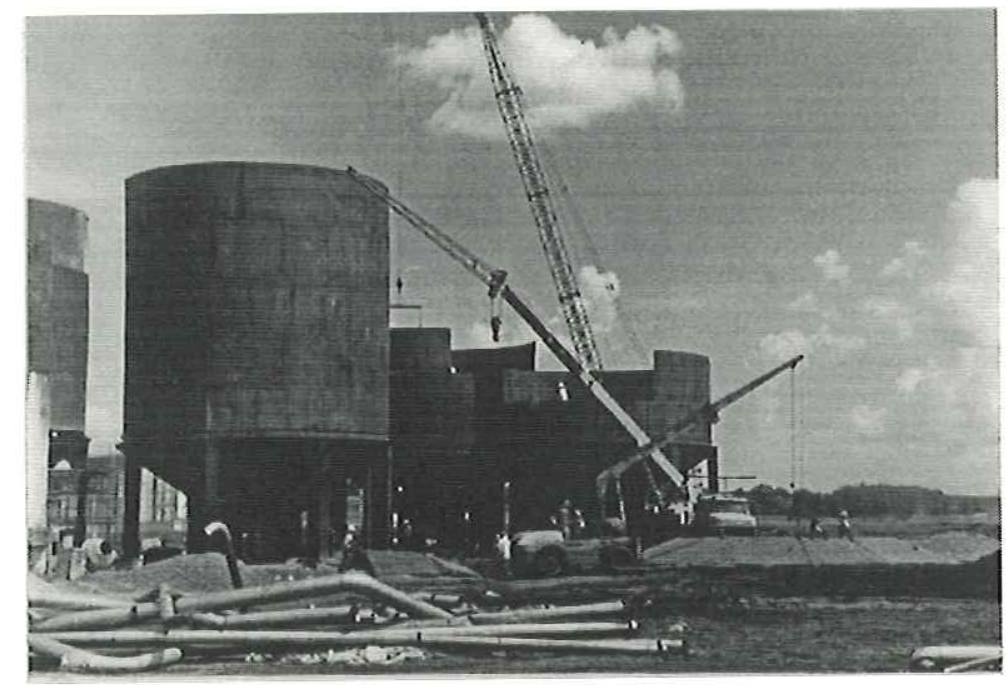

Figura 4 - Estruturas cilíndricas em fase de montagem.

A figura 5 é de um silo metálico, sem tampa, com 27,5 $\mathrm{m}$ de altura e $18,5 \mathrm{~m}$ de diâmetro, situado na Austrália [Ansourian (1992)], que sofreu afundamento do topo devido à ação do vento; a figura 6 mostra silos com amassamento do costado (Ansourian (1992)):

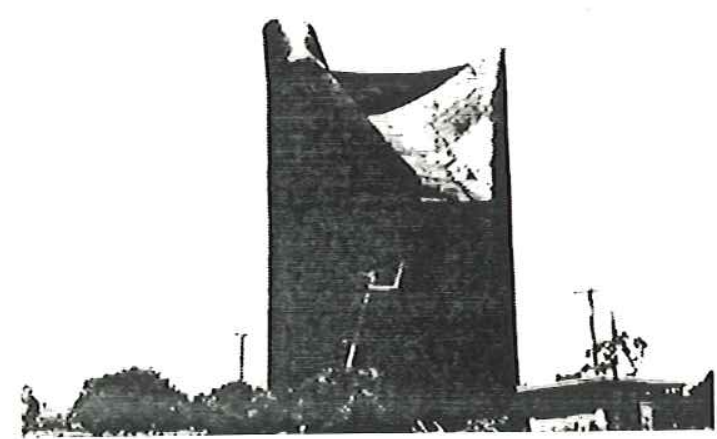

Figura 5 - Afundamento do topo de silo sob a ação do vento.

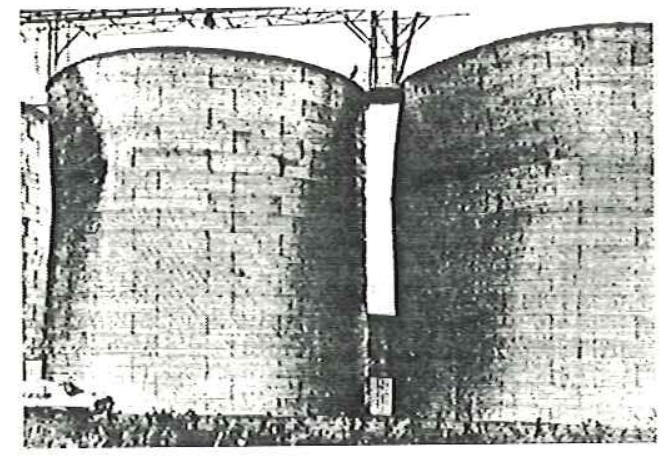

Figura 6 - Amassamento do costado do silo devido à ação do vento.

A preocupação do estudo da perda de estabilidade do equilíbrio de silos e de reservatórios decorre da grande relação diâmetro/espessura da estrutura.

Esta estrutura possui paredes delgadas porque a forma cilíndrica é de grande eficiência para suportar as ações devidas ao material armazenado. Essas ações são axissimétricas e, para este caso, as tensões circunferenciais de membrana são bem 
pela parede de aço, diferentemente das tensões de membrana ao longo da geratriz que, provocadas pelo atrito do material ensilado com a parede, são tensões de compressão (fígura 8) e estão associadas à possível flambagem da parede.

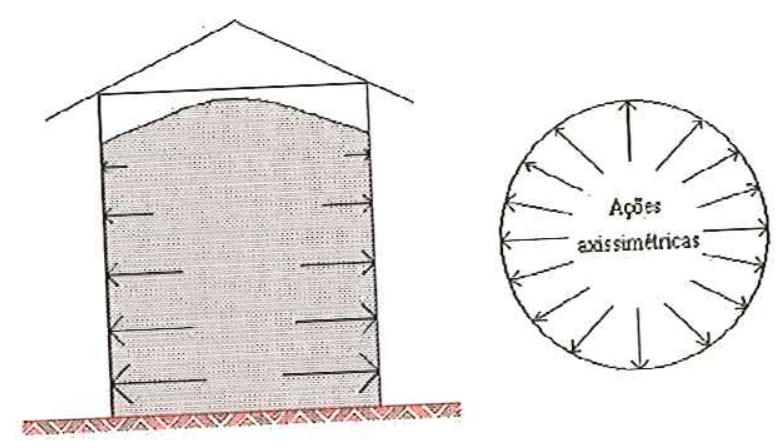

Figura 7 - Ações axissimétricas: seção eficiente.

No entanto, as ações do vento não são axissimétricas e a pequena espessura da parede da estrutura cilíndrica possibilita o afundamento do topo e o amassamento do costado dos silos e dos reservatórios quando se encontram vazios (vide fíguras 5 e 6 ). Além disso, a ação do vento ao longo da geratriz da estrutura também provoca tensões de compressão de membrana, concorrendo para o fenômeno de flambagem (figura 8).

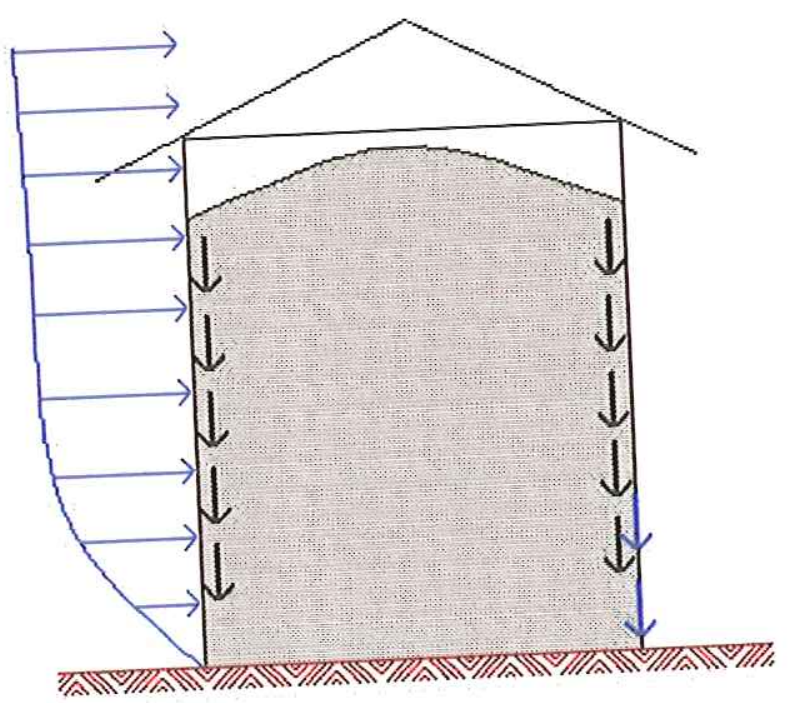

$\downarrow$ : Ações de compressåo devidas ao material ensilado.

$\downarrow$ : Açöes de compressão devidas à açäo do vento.
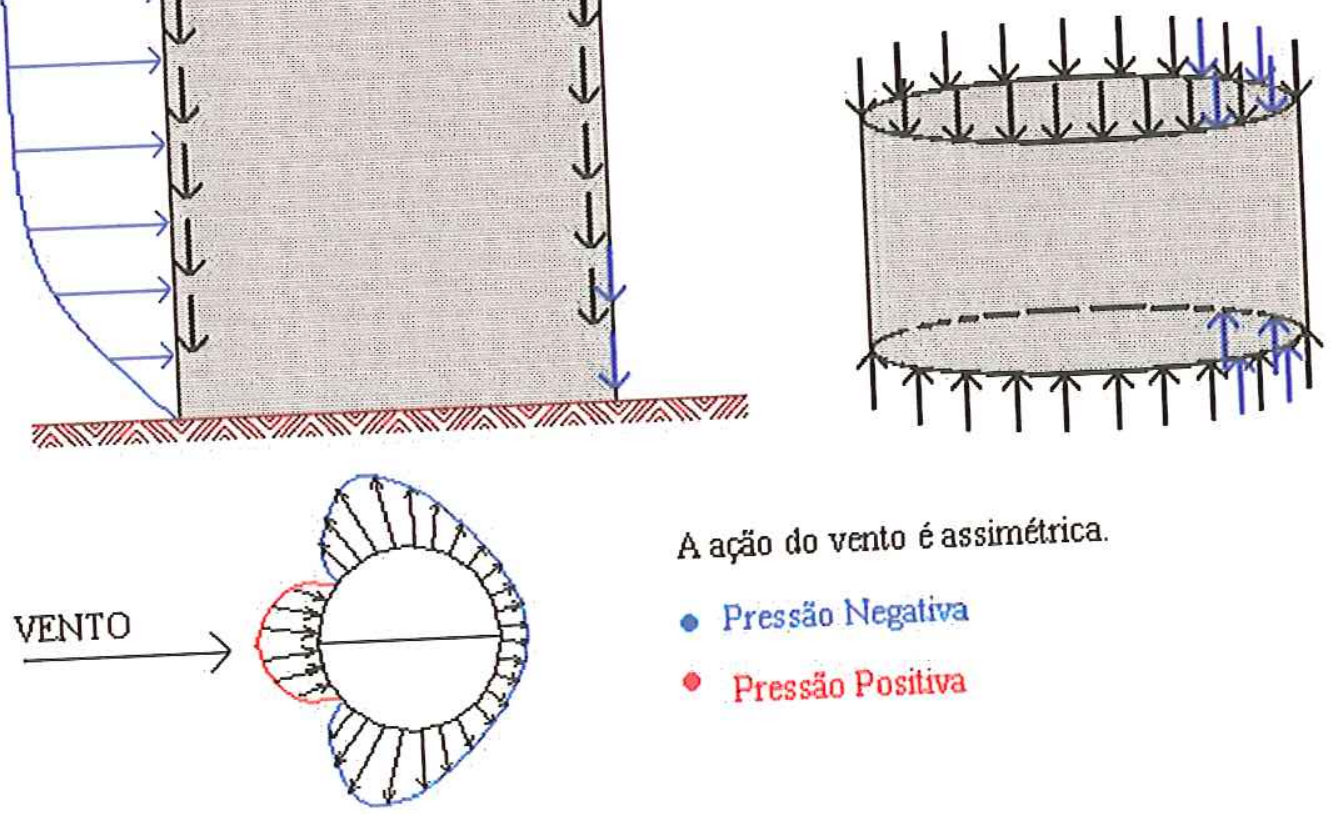

Figura 8 - Tensões meridionais: podem provocar flambagem. 
A flambagem de uma casca cilíndrica pode ser comparada, inicialmente, à flambagem de uma haste delgada; uma vez que as ações de compressão ao longo da geratriz atinjam valores críticos de flambagem na estrutura cilíndrica, podem ocorrer grandes deformações permanentes na casca. Neste estudo de silos e de reservatórios metálicos, em que as paredes cilíndricas podem ser muito esbeltas, é grande a possibilidade de flambagem. Além disto, a estrutura, quando vazia, também está muito suscetível à flexão devida à ação do vento.

A pequena espessura das chapas da parede do silo tem uma importante conseqüência (Bushnell (1984)), uma vez que a resistência da casca cilíndrica à ação de compressão ao longo da geratriz é maior que a resistência à flexão (Figura 9). Por isso, uma casca delgada pode suportar uma solicitação de compressão relativamente grande sem se deformar muito, ou seja, a casca pode absorver uma grande quantidade de energia de deformação de membrana à compressão. Contudo, se houver uma maneira dessa energia de membrana ser convertida em energia de flexão, a casca poderá se deformar muito, podendo perder a estabilidade do equilíbrio, ou seja, flambar.

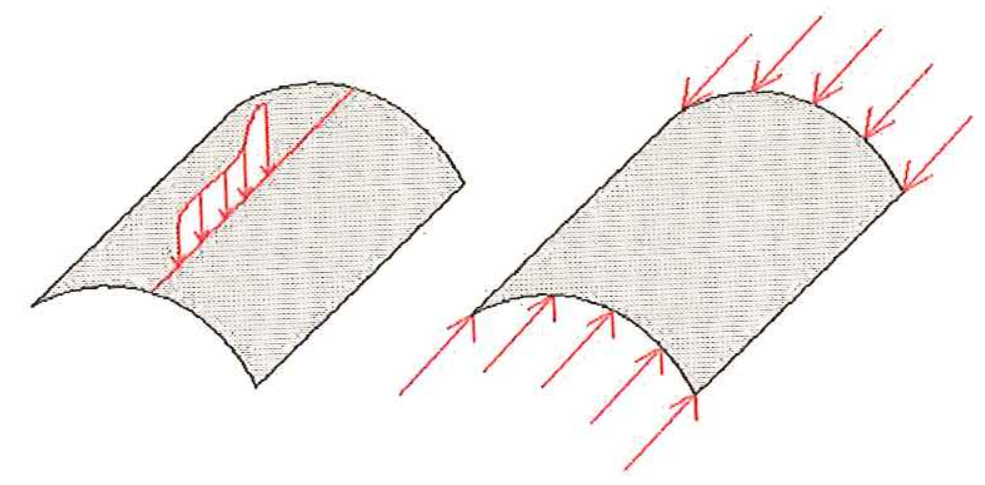

Figura 9 - A resistência à flexão é menor que a resistência à compressão. 


\section{2 - Visão geral do corpo da Dissertação.}

A dissertação compõe-se de 6 capítulos, que, longe de ser um tratado matemático e, portanto, não se propõe a lançar modelos ou novos equacionamentos, visa fornecer informações que permitam ao engenheiro de estruturas a escolha de parâmetros adequados para se evitar o colapso por instabilidade do equilíbrio.

No capítulo 2, é tratado o comportamento estrutural de silos e de reservatórios, a conformação da estrutura e o comportamento de acordo com o tipo de material armazenado. É apresentado ainda o equacionamento dos regimes de membrana e de flexão e,por fím, a maneira como são consideradas as ações devidas aos materiais armazenados e ao vento.

O capítulo 3 está relacionado à análise do problema de estabilidade de cascas cilíndricas. Nele está caracterizado o fenômeno, sendo feitas as distinções para 1) perda de estabilidade devida à compressão axial, 2) perda de estabilidade devida à combinação de compressão axial e pressão interna uniforme (devida ao material granular ou pulverulento), 3) perda de estabilidade devida à combinação de compressão axial e flexão (devida à ação do vento na estrutura vazia).

No capítulo 4 é feita a análise numérica do comportamento estrutural de cascas cilíndricas, para dois modelos, com relações altura/diâmetro de 1:1 e 1:5, sujeitos a ações hidrostática, de material granular ou pulverulento, e do vento, combinadas de acordo com a NBR8681(1984), considerando-se a possibilidade de a estrutura estar vazia e a variação das espessuras das chapas metálicas ao longo da geratriz. Antes da análise numérica em si, é mostrado o tipo elemento finito aplicado e, de modo sucinto, as hipóteses básicas adotadas no programa ANSYSC(1995).

O capítulo 5 apresenta um exemplo de silo metálico cilíndrico para milho, de 1.650 $\mathrm{m}^{3}$, onde se aplica a análise numérica e os conceitos expostos nos capítulos 3 e 4 .

Finalmente, são expostas as conclusões do autor e apontadas as pesquisas que podem ser desenvolvidas na área de silos e reservatórios metálicos. Também estão anexadas as Referências Bibliográficas que despertaram o pensamento e impulsionaram o autor à escrita da dissertação, bem como algumas tabelas, gráficos e textos que, não sendo indispensáveis à dissertação, servem para que se possa reproduzir o exemplo do capítulo 5 e se evitem consultas desnecessárias às referências bibliográficas. 


\section{Capítulo 2 : Comportamento Estrutural e Ações em Silos e em Reservatórios.}

\section{1 - Introdução}

Os silos e os reservatórios são sugeridos, intuitivamente, como estruturas fechadas que servem para armazenar líquidos e materiais agrícolas ou industriais. Neste estudo, o silo pode ser entendido como uma estrutura para armazenar grãos (material granular) ou farinhas (material pulverulento), e o reservatório com a função de armazenar líquidos (água, sucos, óleos vegetais, etc.).

O tipo de silo e de reservatório a ser estudado está caracterizado pela forma cilíndrica, apoiado na superfície do terreno, com fundo plano, tampa plana ou cônica, composto por chapas metálicas calandradas e por chapas planas soldadas entre si. A forma e a nomenclatura básica estão descritas na fígura 10 :

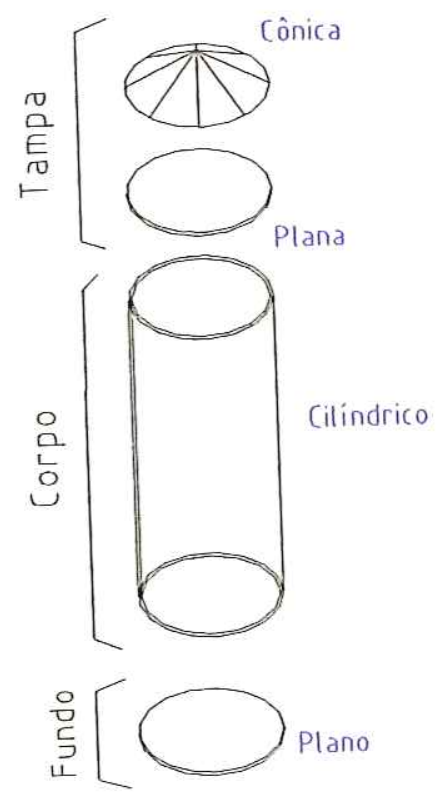

Figura 10 - Partes da Estrutura: Tampa, corpo e fundo. 
a ) O corpo e o fundo são constituídos por chapas metálicas retangulares, calandradas ou planas, soldadas entre si;

b ) A união do corpo com o fundo é feita através de solda ;

c ) Considera-se o corpo cilíndrico apoiado em fundação rígida;

d ) A tampa tem também a função de enrijecer a borda superior do corpo cilíndrico.

A descrição do comportamento estrutural de silos e de reservatórios está fundamentada nas teorias de membrana e flexional das cascas de revolução, com o objetivo de mostrar os deslocamentos e os esforços decorrentes de ações com simetria radial, e cujas hipóteses são :

1 - A estrutura é composta por material homogêneo, isótropo e com relação linear de tensão/deformação;

2 - A espessura e da parede é pequena em relação às dimensões da estrutura;

3 - As retas normais à superfície média permanecem normais à superfície média após a deformação da estrutura;

4 - Os deslocamentos são pequenos em relação à espessura e.

Estas hipóteses são conhecidas como as de Kirchhoff-Love e implicam na validade do princípio da superposição de efeitos, já que a teoria é linear e de $1^{\mathrm{a}}$ ordem. 


\section{2 - Esforços Solicitantes e Equações de Equilíbrio.}

De maneira genérica, um elemento de casca de área $\mathrm{d} S=$ r.d $\mathrm{d}$.dy está submetido a tensões normais $\sigma$ e tangenciais $\tau$ definidas em direção e sentido na figura 11 :

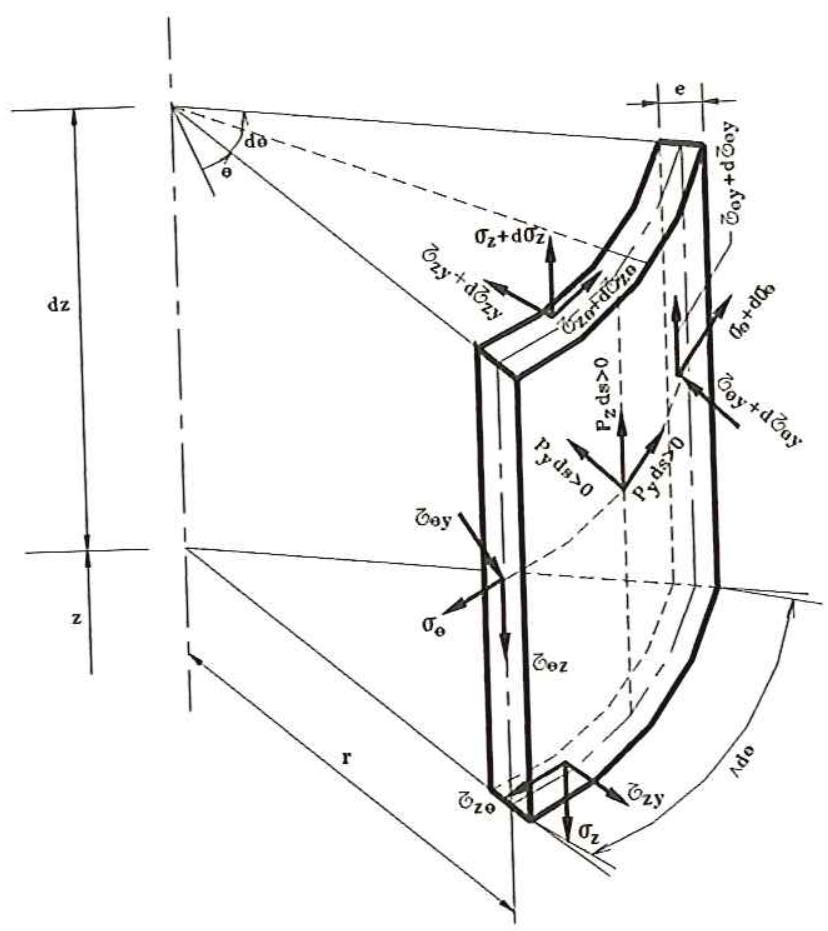

Figura 11 - Esforços Solicitantes Generalizados

Essas tensões resultam em forças e momentos, chamados de esforços solicitantes generalizados, que atuam por unidade de comprimento da face considerada do elemento.

$\mathrm{O}$ cálculo desses esforços generalizados é bastante complexo, pois conduz à resolução de um sistema de equações diferenciais. Contudo, pode-se simplificar o cálculo quando se admite uma distinção dos esforços solicitantes em esforços solicitantes de membrana e de flexão.

Inicialmente, serão apresentados nas figuras 12 e 13 esses esforços de membrana e de flexão e, posteriormente, as condições que caracterizam os regimes de membrana e o regime de flexão: 

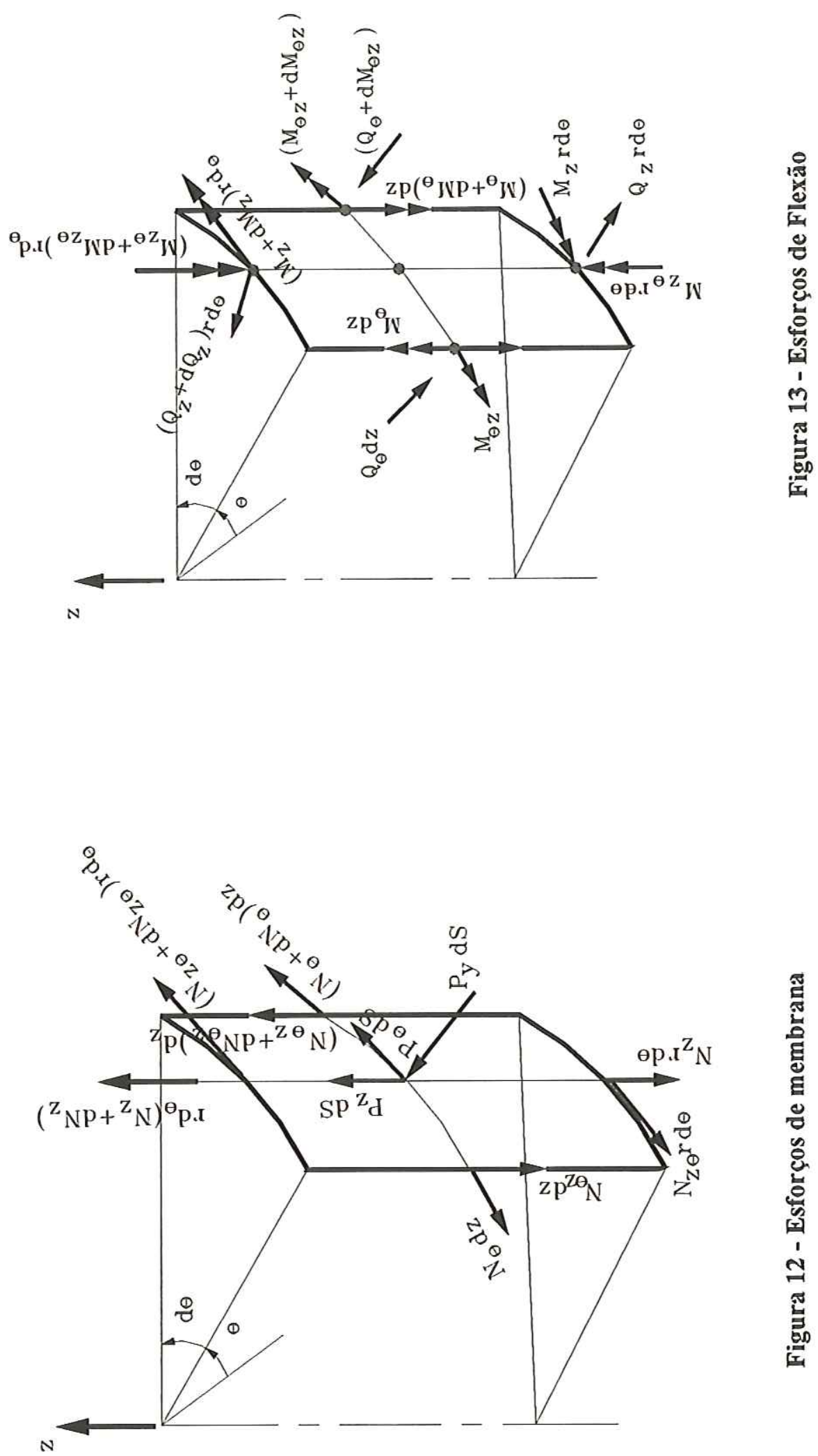

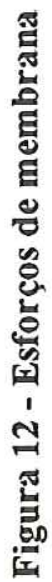


As relações que regem os esforços solicitantes são definidas em função da espessura e das tensões e estão listadas nas equações 1 a 10 :
$N_{\theta}=\int_{-e / 2}^{e / 2} \sigma_{\theta} d y$
eq. 1
$\mathrm{M}_{\mathrm{z}}=\int_{-\mathrm{e} / 2}^{\mathrm{e} / 2} \theta_{\mathrm{z}}\left(1-\frac{\mathrm{y}}{\mathrm{r}}\right) \mathrm{ydy}$
eq. 2
$N_{z}=\int_{-e / 2}^{e / 2} \sigma_{z}\left(1-\frac{y}{r}\right) d y$
eq. 3
$M_{\theta z}=\int_{-e / 2}^{e / 2} \tau_{\theta z} y d y$
eq. 4
$N_{\theta z}=\int_{-e / 2}^{e / 2} \tau_{\theta z} d y$
eq. 5
$\mathrm{M}_{\mathrm{z} \theta}=\int_{-\mathrm{e} / 2}^{\mathrm{e} / 2} \tau_{\mathrm{z} \theta} \mathrm{y}\left(1-\frac{\mathrm{y}}{\mathrm{r}}\right) \mathrm{dy}$
eq. 6
$\mathrm{N}_{\mathrm{z} \theta}=\int_{-\mathrm{e} / 2}^{\mathrm{e} / 2} \tau_{\mathrm{z} \theta}\left(1-\frac{\mathrm{y}}{\mathrm{r}}\right) \mathrm{dy}$
eq. 7
$\mathrm{Q}_{\theta}=\int_{-\mathrm{e} / 2}^{\mathrm{e} / 2} \tau_{\theta \mathrm{y}} \mathrm{dy}$
eq. 8
$\mathrm{M}_{\theta}=\int_{-\mathrm{e} / 2}^{\mathrm{e} / 2} \sigma_{\theta} \mathrm{ydy}$
eq. 9
$\mathrm{Q}_{\mathrm{z}}=\int_{-\mathrm{e} / 2}^{\mathrm{e} / 2} \tau_{\mathrm{zy}}\left(1-\frac{\mathrm{y}}{\mathrm{r}}\right) \mathrm{dy}$
eq. 10

A forma cilíndrica dos corpos dos silos e dos reservatórios e a simetria axial das ações permitem quatro simplificações:
1. $\tau_{\theta y} \equiv 0 \Rightarrow Q_{\theta}=0$
2. $\tau_{\theta z} \equiv 0 \Rightarrow N_{\theta z} \equiv M_{\theta z} \equiv 0$
3. $\tau_{\mathrm{Z} \theta} \equiv 0 \Rightarrow \mathrm{N}_{\mathrm{Z} \theta} \equiv \mathrm{M}_{\mathrm{Z} \theta} \equiv 0$
4. $\mathrm{M}_{\theta} \equiv 0$ 
Essas simplificações diminuem o número de esforços solicitantes de dez para quatro esforços solicitantes: $\mathrm{N}_{\theta}, \mathrm{N}_{\mathrm{Z}}, \mathrm{M}_{\mathrm{Z}} \mathrm{e} \mathrm{Q}_{\mathrm{Z}}$, ilustrados na figura 14:

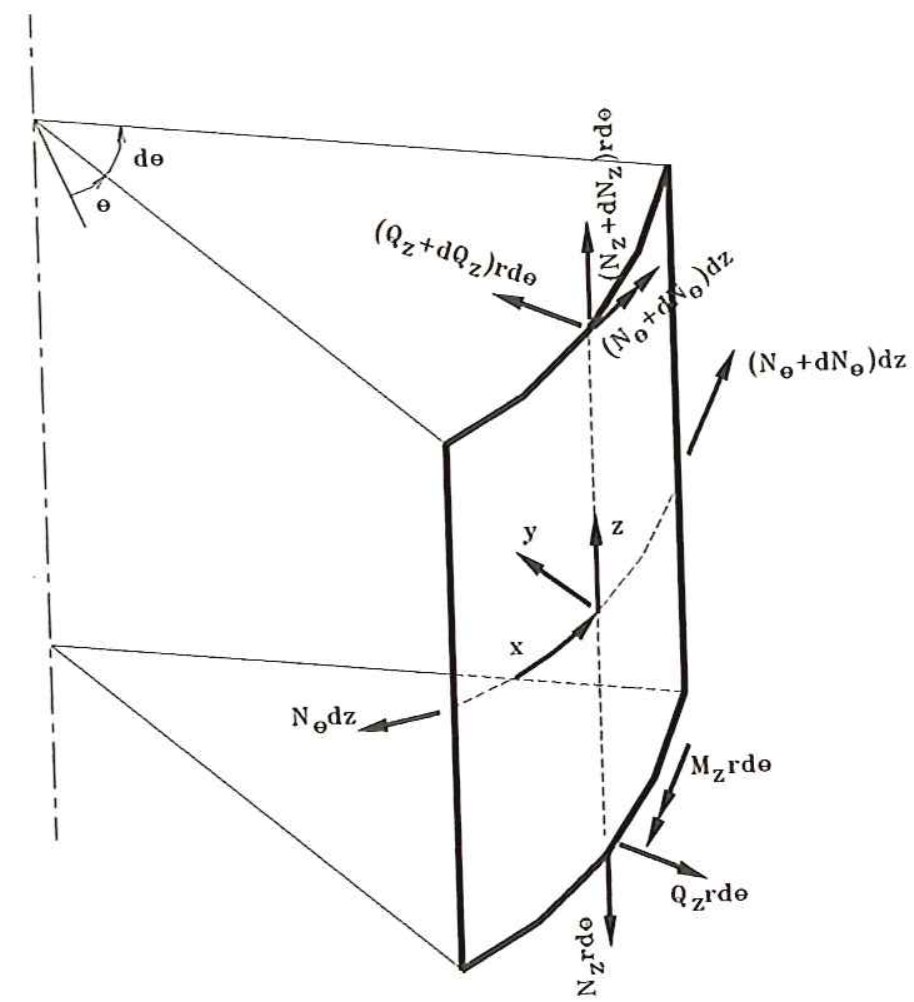

Figura 14 - Esforços solicitantes finais

\section{3 - As Equações de Equilíbrio}

As equações de equilíbrio que resultam das simplificações estão definidas nas direções dos eixos y e $\mathbf{z}$ e em torno do eixo $\mathbf{x}$ :

a) Segundo a direção $\mathbf{z}$ :

$$
\begin{gathered}
-\mathrm{N}_{\mathrm{z}} \mathrm{rd} \theta+\mathrm{p}_{\mathrm{z}} \mathrm{dzrd} \theta+\left(\mathrm{N}_{\mathrm{z}}+\frac{\partial \mathrm{N}_{\mathrm{z}}}{\partial \mathrm{z}} \mathrm{dz}\right) \mathrm{rd} \theta=0 \\
\frac{\partial \mathrm{N}_{\mathrm{z}}}{\partial \mathrm{z}}+\mathrm{p}_{\mathrm{z}}=0
\end{gathered}
$$

eq. 11 
b) Segundo a direção $\mathbf{y}$ :

$$
\begin{gathered}
N_{\theta} d z \frac{1}{2} d \theta+\left[\left(N_{\theta}+d N_{\theta}\right) d z\right] \frac{1}{2} d \theta+p_{y} d z d S+\left(Q_{z}+d Q_{z}\right) r d \theta-Q_{z} r d_{\theta}=0 \\
N_{\theta}+r \frac{\partial Q_{z}}{\partial z}+p_{y} r=0
\end{gathered}
$$

c) Em torno do eixo $\mathbf{x}$ :

$$
\begin{array}{r}
-M_{z} r d \theta-\left(M_{z}+\frac{\partial M_{z}}{\partial z} d z\right) r d \theta+Q_{z} r d \theta d z=0 \\
\frac{\partial M_{z}}{\partial z}-Q_{z}=0
\end{array}
$$

\section{4 - Teoria do Regime de Membrana}

Esta teoria admite que o elemento possui pequena rigidez à flexão e à torção, ou seja, os momentos fletores e os momentos de torção não são significativos em presença dos esforços de compressão.

Desta forma, as cascas apresentam pequena rigidez à flexão e à torção, que despertam um estado de tensões secundárias de pouca importância.

As condições em que se pode aceitar o regime de membrana são [ Gravina (1957) ]:

1. A variação das curvaturas normais da superfície média deverá ser contínua;

2. A variação da espessura da casca deverá ser contínua;

3. A distribuição das forças externas deverá ser contínua;

4. As forças externas aplicadas às bordas livres deverão atuar nos correspondentes planos tangentes à superfície média;

5. As reações dos vínculos deverão estar contidas nos planos tangentes à superfície média.

Essas condições, quando não atendidas, podem provocar uma perturbação, em geral local, e cuja análise deve levar em consideração a rigidez à flexão da casca.

O regime de membrana ocorre quando a casca está sujeita a esforços que atuam ao longo da superfície média, ou seja, apenas aos esforços $\mathrm{N}_{\theta}$ e $\mathrm{N}_{\mathrm{Z}}$. 
Das equações de equilíbrio, uma vez que a equação 11 é independente das equações 12 e 13, pode-se determinar $\mathrm{N}_{\theta}$.

Pela lei de Hooke, aplicada a $\sigma_{\theta}$, ou seja, com $\sigma_{\theta}=\mathrm{E} \varepsilon_{\theta}$, e considerando que a distribuição de $\sigma_{\theta}$ é constante na espessura, isto é, que $\mathrm{N}_{\theta}=\sigma_{\theta}$.e , resulta:

$\varepsilon_{\theta}=\frac{\mathrm{N}_{\theta}}{\mathrm{eE}}$

eq. 14

Sendo o deslocamento radial $w$ função de $\mathrm{z}$ :

$\mathrm{w}(\mathrm{z})=-\varepsilon_{\theta} \mathrm{r}$

eq. 15

Substituindo a equação 3.14 na equação 3.15 :

$\mathrm{w}(\mathrm{z})=-\frac{\mathrm{N}_{\theta}}{\mathrm{eE}} \mathrm{r}$ eq. 16

Ou, observando a equação de equilíbrio em $\mathrm{N}_{\theta}$ :

$$
w(z)=p_{y} \frac{r^{2}}{E e}
$$

\section{5 - Teoria do Regime de Flexão}

O regime de flexão ocorre quando há qualquer alteração nas condições do regime de membrana. Essa alteração provoca uma perturbação, em geral local, e, para avaliá-la, deve-se considerar a rigidez da casca.

Nessa avaliação, são combinadas as equações de equilíbrio e as relações entre esforços e deslocamentos:

$$
\mathrm{N}_{\theta}=-\frac{\mathrm{Ee}}{\mathrm{r}} \mathrm{W}
$$




$$
\begin{aligned}
& M_{z}=-D \frac{d^{2} w}{d z^{2}} \\
& Q_{z}=-D \frac{d^{3} w}{d z^{3}} \\
& M_{\theta}=v M_{z}
\end{aligned}
$$

O resultado dessa combinação, considerando a parede de espessura constante, é uma equação diferencial em função do deslocamento:

$$
\begin{aligned}
& \frac{d^{4} w}{d z^{4}}+4 \beta^{4} w=\frac{p_{y}}{D} \\
& \operatorname{com} D=\frac{E e^{3}}{12\left(1-v^{2}\right)} ; \beta^{4}=\frac{3\left(1-v^{2}\right)}{r^{2} e^{2}} \Rightarrow \beta \approx \frac{1,3}{\sqrt{r e}}
\end{aligned}
$$

A solução geral da equação diferencial22 é composta pelas solução particular wp $\mathrm{e}$ homogênea $w_{h}$ :

$$
w(z)=w_{h}(z)+w_{p}(z)
$$

A solução homogênea está defínida por:

$w_{h}(z)=\exp (\beta z)\left(C_{1} \cos \beta z+C_{2} \operatorname{sen} \beta z\right)+\exp (\beta z)\left(C_{3} \cos \beta z+C_{4} \operatorname{sen} \beta z\right)$

E a solução particular para pressão hidrostática:

$$
w_{p}(z)=p_{y} \frac{r^{2}}{E e}
$$

Desta maneira, os deslocamentos da parede do reservatório podem ser calculadas com: 


$$
w(z)=p_{y} \frac{r^{2}}{E e}+\exp (\beta z)\left(C_{1} \cos \beta z+C_{2} \operatorname{sen} \beta z\right)+\exp (-\beta z)\left(C_{3} \cos \beta z+C_{4} \operatorname{sen} \beta z\right) \quad \text { eq. } 26
$$

As condições de vinculação do reservatório são as que definem os valores das constantes $\mathrm{C}_{1}, \mathrm{C}_{2}, \mathrm{C}_{3}, \mathrm{C}_{4}$.

Vale ressaltar que, no presente estudo, esse cálculo de w(y) é simplificado porque os reservatórios são relativamente longos H/D > 1 e suas paredes são muito delgadas $(r / e>200)$. Isto significa afirmar que as perturbações do fundo do reservatório não alcançam o topo e $\mathrm{C}_{1}$ e $\mathrm{C}_{2}$ podem ser admitidas nulas. Analogamente, as perturbações do topo não alcançam o fundo do reservatório.

Observe-se que, estando as funções trigonométricas multiplicadas por termos exponenciais, existe uma tendência de redução no valor do termo correspondente a $\exp (-\beta z)$ à medida em que o valor de $\mathrm{z}$ aumenta $\mathrm{e}$, quanto maior o valor de $\beta$, mais rápida é essa redução. Deste fato, $\beta$ é uma característica da casca cilíndrica chamada decoeficiente de amortecimento.

Uma maneira prática de verificar se os efeitos de uma borda não afetam a outra borda do silo ou reservatório é verificar se o valor de $\beta \mathrm{H} \geq 5$ [BILLINGTON(1965)]. As figuras 15 e 16 revelam, para diversas relações raio/espessura, como varia o valor deß $\mathrm{H}$. Para a figura 15 foi considerado o reservatório de aço, paredes delgadas, $265<\mathrm{r} / \mathrm{e}<1000, \mathrm{r}=4,0 \mathrm{~m}$, $4<\mathrm{e}<15 \mathrm{~mm}, \mathrm{H}=4,0 \mathrm{~m} \mathrm{e} \mathrm{H}=8,0 \mathrm{~m}$, e para a figura 16 foi considerado o reservatório de concreto, $13<\mathrm{r} / \mathrm{e}<40, \mathrm{r}=4,0 \mathrm{~m}, 100<\mathrm{e}<320 \mathrm{~mm}, \mathrm{H}=4,0 \mathrm{~m}$ e $\mathrm{H}=8,0 \mathrm{~m}$ :

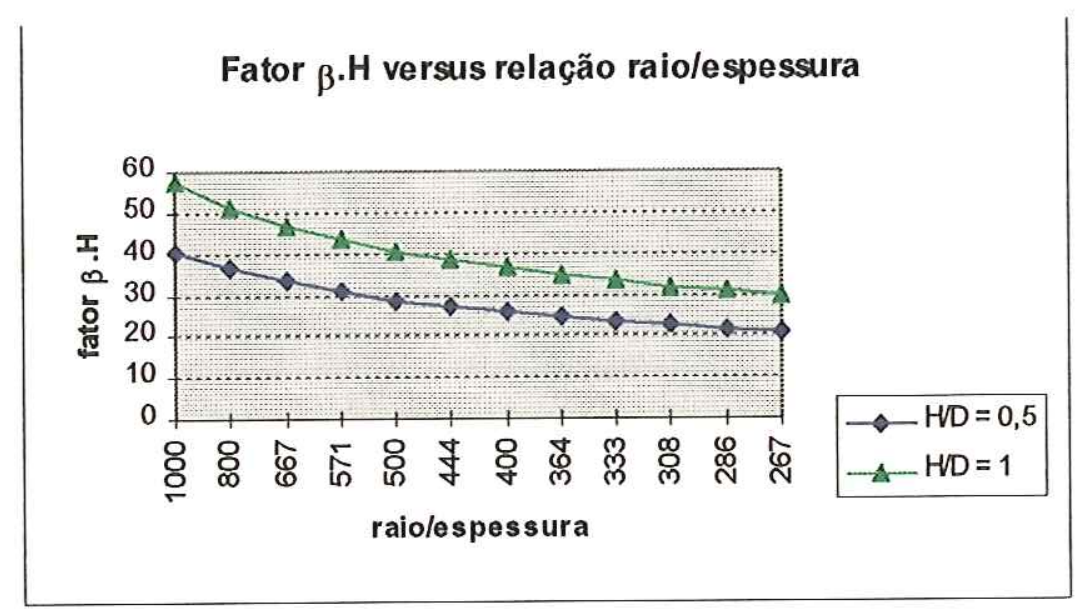

Figura 15 - Fator $\beta . H$ para reservatórios de aço 


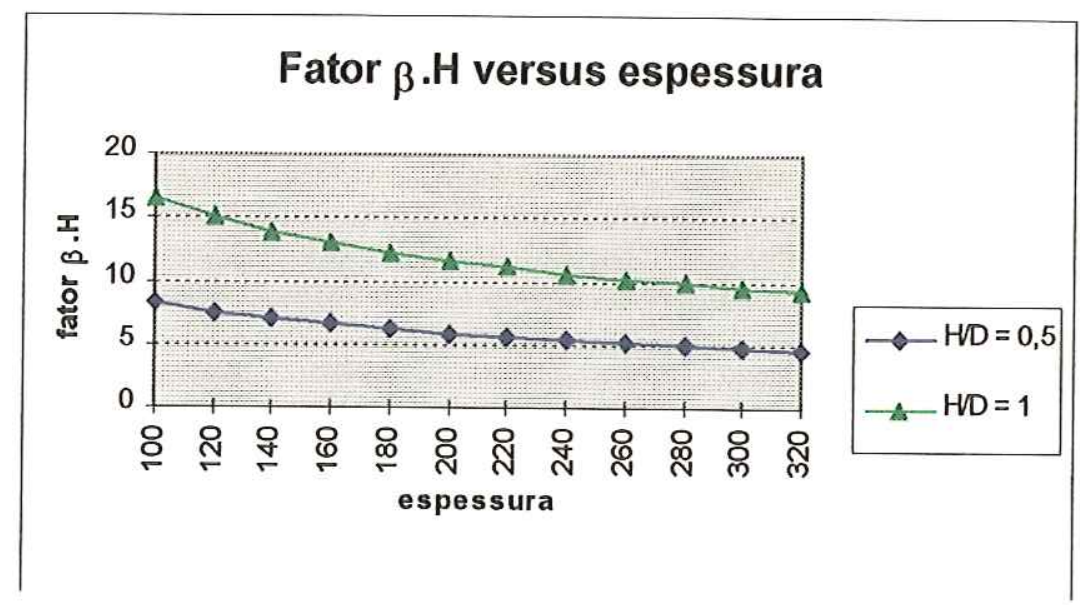

Figura 16 - Fator $\beta . H$ para reservatórios de concreto

Observando-se os gráficos nas figuras 15 e 16, percebe-se que, para as relações altura/diâmetro 0,5 e 1,0 , é coerente serem considerados nulos os coeficientes $\mathrm{C}_{1}$ e $\mathrm{C}_{2}$, principalmente nos reservatórios em aço. Para relações H/D $>1,0$ há uma distância maior para o amortecimento e, nas estruturas de silos em aço, os efeitos de uma borda não afetam a outra borda (as paredes são muito delgadas).

\section{6 - As ações}

As ações consideradas são as do peso próprio da estrutura, do material armazenado, e a do vento. Não são consideradas ações provenientes da fermentação do material ensilado, ou da liberação de gases ( caso dos reservatórios de petróleo ), bem como de equipamentos especiais. Isto porque se admite que esses tipos de ações não provocam esforços significativos na análise da estabilidade da estrutura, exceto em condições em que o equipamento aumente em muito o valor da tensão meridional de membrana.

As ações e as estruturas dos reservatórios serão analisadas de acordo com a simbologia e os desenhos indicados na figura 17 - Nomenclatura dos Silos, que se baseia na terminologia das NBR 11162(1990) e NBR 11165(1990), e na figura 18 - Esquema de ações. 


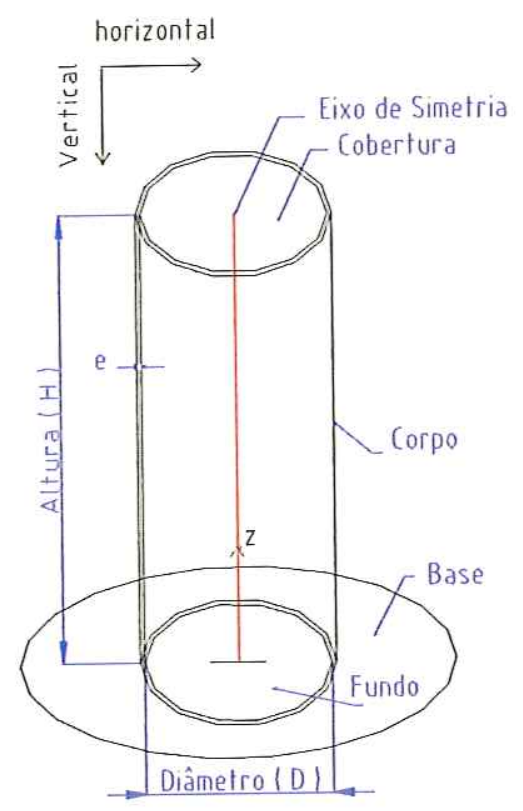

Figura 17 - Nomenclatura dos Silos

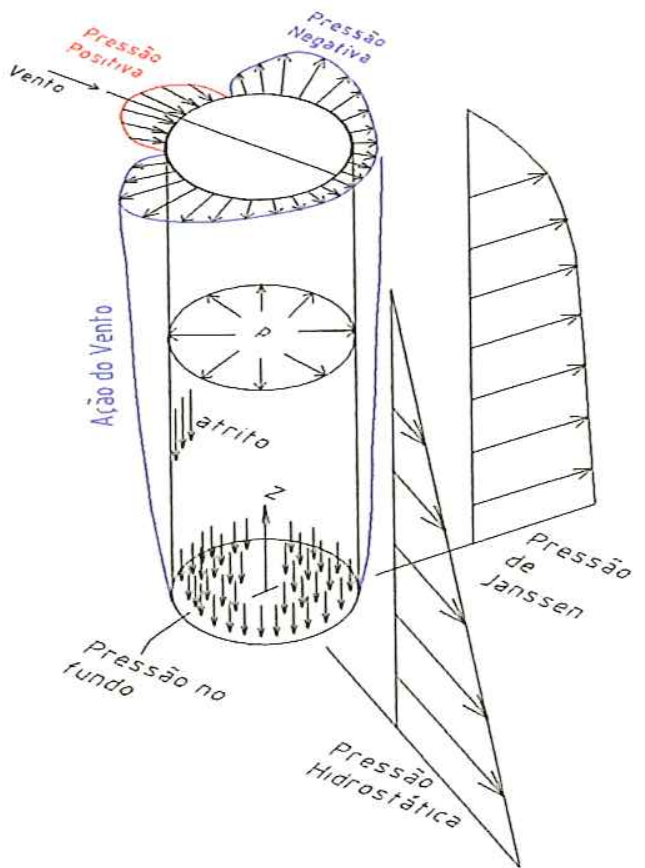

Figura 18 - Esquema de ações

\subsection{1 - A Ação do Vento}

A ação variável do vento está calculada segundo a NBR6123 -Forças devidas ao Vento em Edificaçóes (1987) e, como regra geral, assume-se que o vento pode atuar em qualquer direção horizontal. Como a estrutura do reservatório é axissimétrica em relação a um eixo perpendicular à direção do vento, considera-se que o vento pode incidir perpendicularmente a qualquer geratriz do reservatório.

A componente estática de pressão do vento, $\Delta \mathrm{P}$, que atua perpendicularmente sobre um elemento de área, é dada por:

$$
\Delta \mathbf{P}=\mathbf{C}_{\text {pe.q.dA }}
$$

- Sendo $\mathrm{C}_{\mathrm{pe}} \mathrm{o}$ coeficiente de pressão externa. 
Os coeficientes de pressão externa $C_{\text {pe }}$ são expressos para o corpo da estrutura, figura 20 (coeficientes extraídos da NBR6123(1987)), e para a cobertura cônicaf̧igura 22 ( BRIASSOULIS (1986)), e a cobertura plana, figura 21 (Esslinger et al. (1971)).

E q é a pressão do vento em um ponto onde ocorre a estagnação do ar, obtida da expressão $q=0,613 \cdot V_{k}{ }^{2}, V_{k}=S_{1} \cdot S_{2} \cdot S_{3} \cdot V_{0}, V_{k} \mathrm{em} \mathrm{m} / \mathrm{s}$ e q em N/m².

A velocidade $\mathrm{V}_{0}$ é chamada velocidade básica, correspondente a uma rajada de 3 segundos, excedida em média uma vez em 50 anos, medida a $10 \mathrm{~m}$ acima do terreno, em local plano e aberto; as isopletas de velocidades básicas, em $\mathrm{m} / \mathrm{s}$, encontram-se na figura $\mathrm{A}$ (anexo 1 deste trabalho) da NBR 6123 (1987). Para os exemplos, adotou-se $\mathrm{V}_{0}=40 \mathrm{~m} / \mathrm{s}$.

$\mathrm{O}$ fator topográfico $\mathrm{S}_{1}$ é utilizado na avaliação das variações do relevo do terreno, e adotado igual a 1,0 para os modelos numéricos e no exemplo do capítulo 5 .

$\mathrm{O}$ fator $\mathrm{S}_{2}$ serve para a consideração do"efeito combinado da rugosidade do terreno, da variação da velocidade do vento com a altura acima do terreno e das dimensões da edificação"( NBR6123 (1987) ).

$\mathrm{O}$ fator $\mathrm{S}_{2}$ é calculado para uma altura $\mathrm{z}$, medida acima da superfície nivelada do terreno, com a expressão:

$$
S_{2}=b . F_{r} \cdot(z / 10) p
$$

sendo $\mathrm{b}=$ parâmetro meteorológico, $\mathrm{p}=$ expoente de variação de $\mathrm{z} / 10, \mathrm{~F}_{\mathrm{r}}=$ fator de rajada do vento.

E o fator estatístico $S_{3}$, que avalia o grau de segurança e a vida útil da estrutura, consideradas as instalações de silos e de reservatórios com baixo fator de ocupação humana, foi tomado igual a 0,95 . 

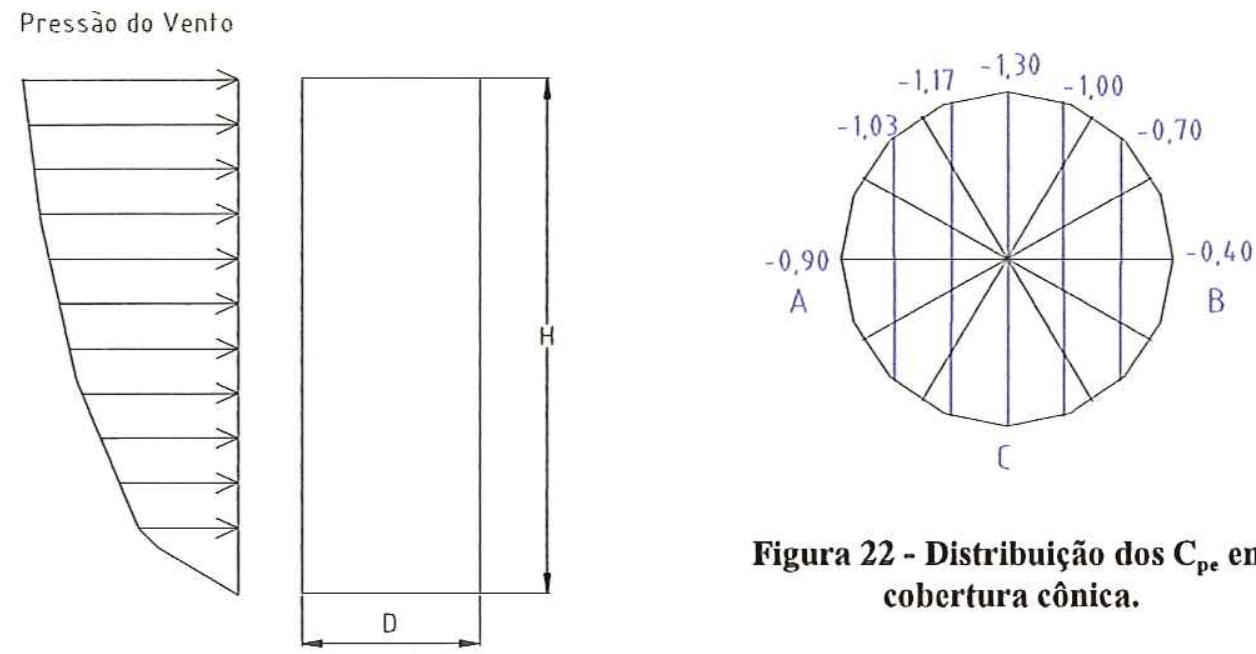

Figura 22 - Distribuição dos $\mathrm{C}_{\mathrm{pe}} \mathrm{em}$ cobertura cônica.

Figura 19 - Pressão ao longo da altura do silo.

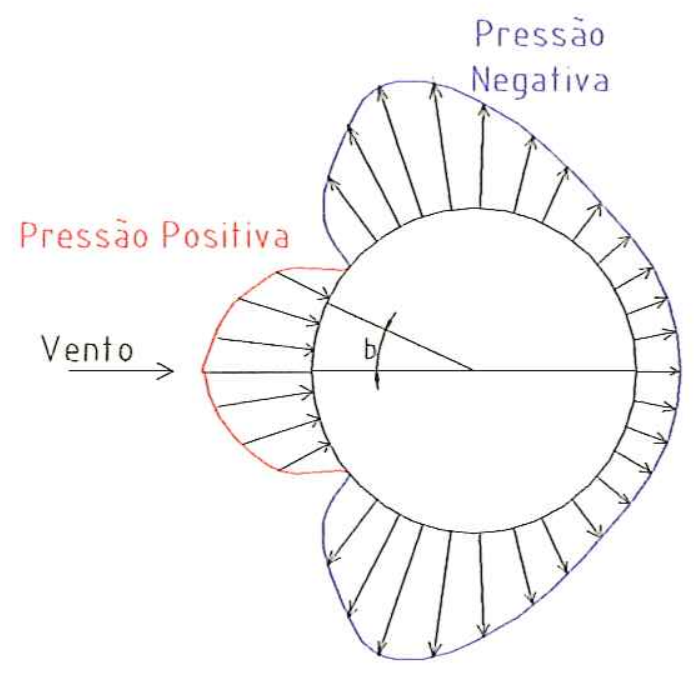

Figura 20 - Configuração dos coeficientes $C_{\mathrm{pe}}$ no perímetro do silo.

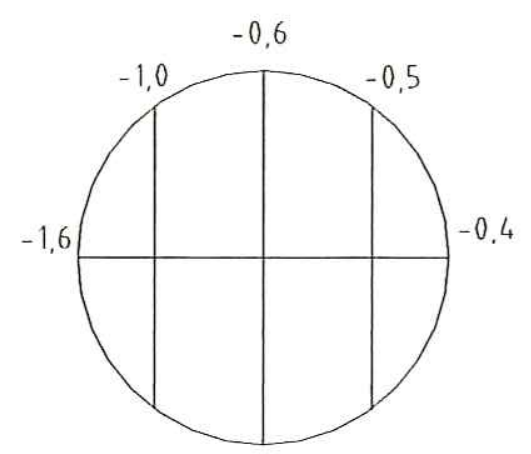

Figura 21 - Distribuição dos $\mathrm{C}_{\text {pe }}$ em Cobertura

\begin{tabular}{|c|c|c|}
\hline \multicolumn{3}{|c|}{$\begin{array}{c}\text { TABELA I - Valores dos C pe } \\
\text { nerimetro do Silo. }\end{array}$} \\
\hline $\mathrm{b}$ & $\mathrm{H} / \mathrm{D} \leq 2,5$ & $\mathrm{~A} / \mathrm{D}=10$ \\
\hline 0 & $+1,0$ & $+1,0$ \\
\hline 10 & $+0,9$ & $+0,9$ \\
\hline 20 & $+0,7$ & $+0,7$ \\
\hline 30 & $+0,35$ & $+0,35$ \\
\hline 40 & 0 & 0 \\
\hline 50 & $-0,5$ & $-0,7$ \\
\hline 60 & $-1,05$ & $-1,2$ \\
\hline 70 & $-1,25$ & $-1,4$ \\
\hline 80 & $-1,3$ & $-1,45$ \\
\hline 90 & $-1,2$ & $-1,4$ \\
\hline 100 & $-0,85$ & $-1,1$ \\
\hline 120 & $-0,4$ & $-0,6$ \\
\hline 140 & $-0,25$ & $-0,35$ \\
\hline 160 & $-0,25$ & $-0,35$ \\
\hline 180 & $-0,25$ & $-0,35$ \\
\hline & & \\
\hline
\end{tabular}

\section{Plana}




\subsection{2 - As Ações devidas ao Material Sólido}

As ações devidas aos materiais granulares ou pulverulentos serão avaliadas de acordo com a norma alemã de ações em silos DIN1055-6 (1986). O seu campo de aplicação, de modo geral, está definido para silos com células verticais, prismáticas ou cilíndricas, com relação altura/diâmetro pelo menos igual a 0,8 , com tremonha ou fundo plano, material granular ou pulverulento, sem coesão.

A altura máxima do silo, que pode ser analisada pela norma, é limitada pela relação entre a pressão vertical total do material ensilado resultante no fundo do silo, qf , e o peso específico do material ensilado $\gamma$ :

$$
\mathrm{q}_{\mathrm{f}} / \gamma \leq 25 \mathrm{~m}
$$

eq. 29

O tipo de silo a ser analisado é do tipo cilíndrico, de superfície, vertical e de fundo plano, que geralmente provoca um tipo de escoamento (do material ensilado ) chamadofluxo de funil, onde parte do material permanece em repouso:

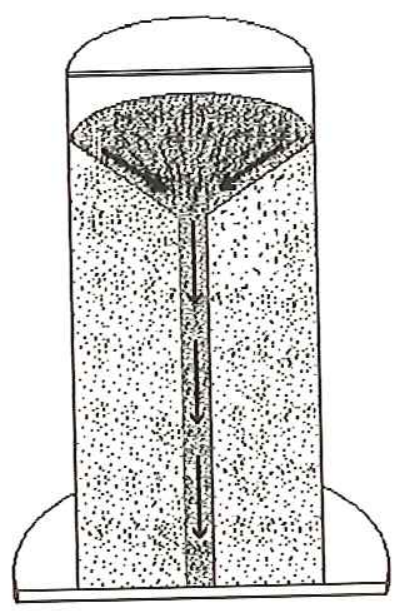

Figura 23 - Fluxo de funil

A Figura 23 ilustra um fluxo de funil com conduto estável. Do contrário, quando ocorre um fluxo de saída mais um fluxo de entrada, pode haver formação de um conduto instável e, conseqüentemente, sobrepressões dinâmicas elevadas. 
Na formulação para o cálculo das pressões, da força de atrito na parede e na descrição dos parâmetros relacionados ao material ensilado, a nomenclatura básica é:

$\gamma \quad$ : peso específico do material ensilado

$\phi \quad$ : ângulo de atrito entre as partículas do material ensilado ( ângulo de atrito interno)

$\phi$ ' ângulo de atrito entre o material ensilado e as paredes da célula

$\mu=\operatorname{tg} \phi$ : coeficiente de atrito entre as partículas do material ensilado (coef. de atrito interno)

$\mu^{\prime}=\operatorname{tg} \phi '$ : coefíciente de atrito entre o material ensilado e as paredes da célula

q : : : pressão vertical atuando na direção do eixo do silo

p : : pressão horizontal atuando na parede do silo

$\mathrm{v} \quad$ : força de atrito na parede por unidade de área

$\mathrm{S} \quad$ : área da seção transversal da massa ensilada

$\mathrm{U} \quad$ : perímetro da seção $\mathrm{S}$

$\mathrm{R}=\mathrm{S} / \mathrm{U}$ : raio hidráulico da célula

D : : diâmetro da seção transversal

H : altura total da célula

e : espessura da parede

$\mathrm{K}_{\mathrm{d}}$ : fator dinâmico considerado quando da descarga do silo

$\lambda=\mathrm{p} / \mathrm{q}$ : razão entre as pressões horizontal e vertical

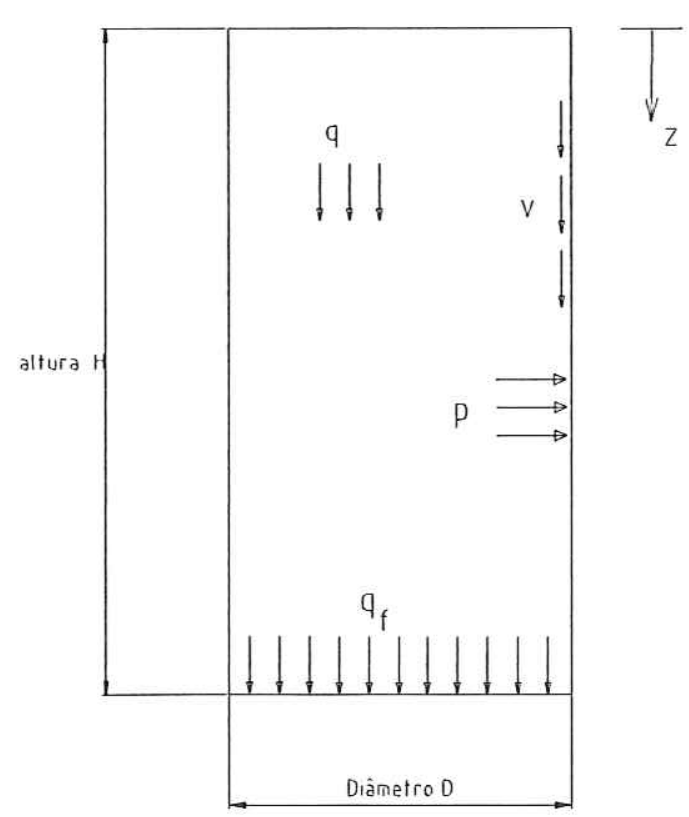

Figura 24 - Ações do material no silo 
Inicialmente são expostas as expressões da norma alemã DIN1055-6 (1986) para o cálculo das pressões estáticas, lateral e vertical, e da força de atrito na parede por unidade de área e, posteriormente, os efeitos das sobrepressões na descarga dos silos.

Quando do enchimento, até a situação de repouso do material ensilado, as pressões lateral e vertical e a força de atrito por unidade de área podem ser calculadas com:

$$
\begin{array}{ll}
\text { atrito } & V_{c}=\frac{\gamma \cdot S}{U} \phi(z) \\
\text { lateral } & p_{C}=\frac{\gamma \cdot S}{\mu^{\prime} U} \phi(z) \\
\text { vertical } & q_{c}=\frac{\gamma \cdot S}{\lambda \cdot \mu^{\prime} \cdot U} \phi(z)
\end{array}
$$

Onde a função $\phi(\mathrm{z})$ é dada por:

$$
\begin{aligned}
& \phi(z)=\left(1-e^{-z / z_{0}}\right) \\
& z_{0}=\frac{S}{\lambda \cdot \mu^{\prime} \cdot U}
\end{aligned}
$$

Da superfície superior do material armazenado até uma profundidade $\mathrm{z}$, a resultante do atrito, em unidade de força por unidade de comprimento, é dada por:

$$
\mathrm{V}_{\mathrm{cs}}=\frac{\gamma \cdot \mathrm{S}}{\mathrm{U}}\left[\mathrm{z}-\mathrm{z}_{0} \cdot \phi(\mathrm{z})\right]
$$

A pressão qf que atua no fundo plano do silo é considerada constante sobre toda a seção transversal e, para silos com $H / D \geq 1,5$ :

$$
\begin{aligned}
& \mathrm{q}_{\mathrm{f}}=\mathrm{C}_{\mathrm{f}} \mathrm{q}_{\mathrm{c}} \leq \gamma \cdot \mathrm{H} \\
& \text { com } \quad \mathrm{C}_{\mathrm{f}}=1,5 \text { ( grãos em geral) } \\
& \mathrm{C}_{\mathrm{f}}=1,8 \text { (material pulverulento) }
\end{aligned}
$$


Quando do esvaziamento do silo as ações atuam dinamicamente, mas para a consideração estática dos efeitos essas ações são multiplicadas por fatores de equivalência do efeito dinâmico para a ação estática. Para um esvaziamento uniforme, valem as relações:

$$
\begin{array}{lr}
\mathrm{V}_{\mathrm{d}}=1,1 \cdot \mathrm{v}_{\mathrm{c}} & \text { eq. } 37 \\
\mathrm{p}_{\mathrm{d}}=\mathrm{e}_{\mathrm{h}} \cdot \mathrm{p}_{\mathrm{c}} & \text { eq. } 38 \\
\mathrm{q}_{\mathrm{c}}=1,25 . \mathrm{q}_{\mathrm{d}} & \text { eq. } 39
\end{array}
$$

O fator 1,1 também é válido para o somatório da pressão de atrito ao longo da altura $\left(\mathrm{v}_{\mathrm{cs}}\right)$. O fator $\mathbf{e}_{\mathbf{h}}$ encontra-se no apêndice, em tabela extraída da DIN1055-6 (1986).

No caso de um esvaziamento não uniforme, considerando que o topo e o fundo tenham enrijecedores de borda, os valores da pressão horizontal $\mathrm{p}_{\mathrm{c}}$ são majorados por um fator $\mathrm{C}_{\mathrm{m}}$ :

$\mathrm{C}_{\mathrm{m}}$ : fator de sobrepressão, que, para silos circulares com $\mathrm{D} / \mathrm{e} \geq 200$ (e: espessura da parede do silo):

$$
\mathrm{Cm}=1,0+3 \mathrm{C} \sqrt{ }(\mathrm{H} / \mathrm{D})
$$

$\mathrm{O}$ fator $\mathrm{C}=\mathrm{C}_{\mathrm{h}} \cdot \mathrm{C}_{\mathrm{e}} \cdot \mathrm{C}_{\mathrm{r}} \cdot \mathrm{C}_{\mathrm{g}}$

$\mathrm{C}_{\mathrm{h}} \quad$ : fator de altura $-1,0 \leq \mathrm{C}_{\mathrm{h}}=(0,13 \cdot \mathrm{H} / \mathrm{D}+0,87) \leq 1,4$

$\mathrm{C}_{\mathrm{e}} \quad$ : fator de excentricidade $-1,0 \leq \mathrm{C}_{\mathrm{e}}=0,5(1+3 \mathrm{e} / \mathrm{r}) \leq 2,0$

e $\quad$ : excentricidade do ponto de descarga em relação ao eixo vertical do silo cilíndrico

$\mathrm{C}_{\mathrm{r}} \quad$ : fator de rigidez $-\mathrm{C}_{\mathrm{r}}=0,05$ para $\mathrm{D} / \mathrm{t} \leq 200$

$\mathrm{C}_{\mathrm{g}}$ : fator que depende do material ensilado (vem tabelado na DIN 1055)

A pressão lateral total no esvaziamento do silo é dada por:

$$
\mathrm{p}_{\mathrm{esv}}=\mathrm{C}_{\mathrm{m}} \cdot \text { (pressão estática de Janssen) }
$$


Para as considerações do acréscimo das ações devido aos impactos do material, impactos oriundo da formação e desmoronamento de cúpulas, há uma majoração da pressão horizontal $\mathrm{p}_{\mathrm{c}}$, ao longo de toda a altura, por um fator $\mathrm{C}_{\mathrm{S}}$ :

$$
\mathrm{C}_{\mathrm{s}}=1+\frac{0,50}{\sqrt{\mathrm{k}}}
$$

eq. 41

$\mathrm{k}$ : distância entre o eixo vertical da abertura de saída e a parede do silo (mínimo k =0,50 m).

\subsection{3 - A Ação Hidrostática.}

A ação hidrostática provoca efeitos que atuam nas direções radial e vertical e resultam em pressão lateral no costado e pressão no fundo do reservatório:

$\begin{array}{ll}\text { lateral } & \mathrm{p}=\boldsymbol{\gamma} \cdot \mathrm{z} \\ \text { fundo } & \mathrm{qf}_{\mathrm{f}}=\gamma \cdot \mathrm{H}\end{array}$

eq. 42

eq. 43 


\section{Capítulo 3 - Análise do problema de estabilidade em cascas cilíndricas.}

\section{1 - Introdução}

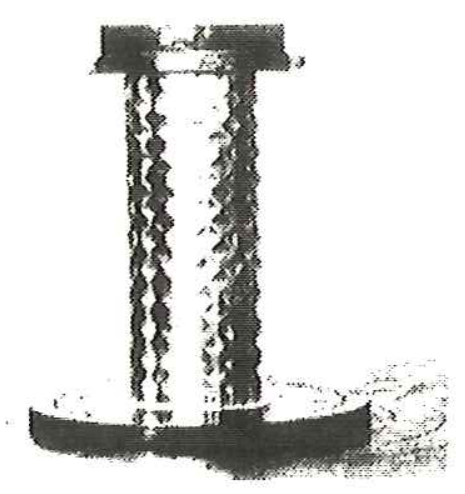

Figura 25- Configuração de flambagem elástica de cilindro comprimido

A forma cilíndrica para as estruturas de silos e de reservatórios é uma das mais eficientes para o armazenamento de grandes volumes de material líquido ou granular. Devido a essa grande eficiência, as chapas metálicas que formam o costado da estrutura são muito finas em relação ao diâmetro (estrutura de paredes delgadas), e a questão que surge então é até que ponto o equilíbrio dessa estrutura é estável.

A relação da espessura para o raio do cilindro é, portanto, um parâmetro significativo no estudo dessa estabilidade. Considera-se também a relação altura para o diâmetro, bem como a maneira como são aplicados os sistemas de forças que atuam nessas estruturas.

Vale esclarecer que o sistema está em equilíbrio estável se, depois de impostas perturbações tais como pequenas mudanças de posição, a configuração resultante encontra-se próxima da configuração inicial. 
Existem maneiras diferentes de uma casca cilíndrica perder a estabilidade do equilíbrio, dependentes de vários fatores. Os cilindros longos (relação altura/diâmetro > 3) perdem a estabilidade do equilíbrio, ou flambam, em um modo periódico de deformação na circunferência, chamado modo assimétrico, como ilustra a figura 26 , quando a ação dominante é uma pressão externa provocada, por exemplo, pela ação do vento:

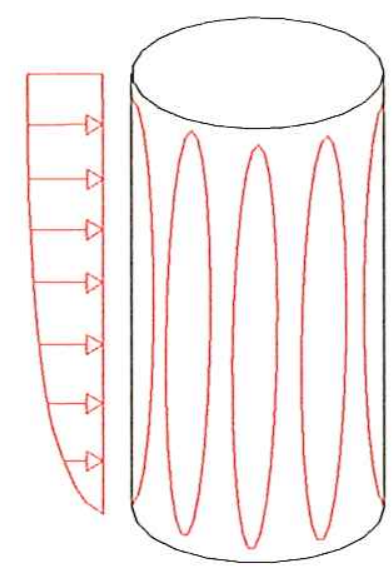

Figura 26 - Modo geral de flambagem periódica na circunferência de um cilindro longo

Se a pressão externa não for dominante e houver, suponha-se, uma compressão axial, este modo de flambagem pode adquirir formas diferentes daquela ilustrada na figura 26 . Esta suposição, entretanto, não fará parte da análise que será efetuada neste trabalho, porque somente cabe ser analisado, para uma pressão externa dominante (a ação do vento), o caso do silo ou do reservatório quando estiver vazio.

Quando o silo estiver cheio, o modo de flambagem que pode ocorrer é o modo axissimétrico de flambagem local, ilustrado pela figura 27 , e provocado pela ação resultante do atrito entre o material ensilado e o costado do silo:

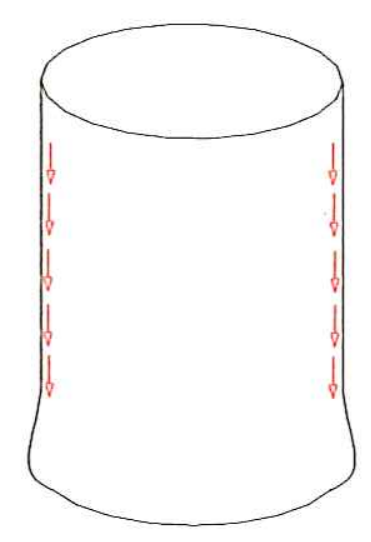

Figura 27 - Modo axissimétrico de flambagem local. 
A maneira como a estrutura é montada pode contribuir para a flambagem no modo axissimétrico. Em geral, as estruturas são montadas com chapas calandradas dispostas de modo a formarem um anel e cada anel é soldado em cima de outro anel, na direção da geratriz, de modo que um silo fica composto de vários anéis. As tensões residuais, oriundas dos processos de soldagem e de conformação (calandragem) das chapas metálicas utilizadas nos silos, contêm componentes axissimétricos que podem ocasionar uma flambagem que se manifesta a um valor abaixo do valor da tensão crítica de flambagem prevista. A figura 28 ilustra como as chapas são dispostas e as regiões onde ocorrem as tensões residuais.

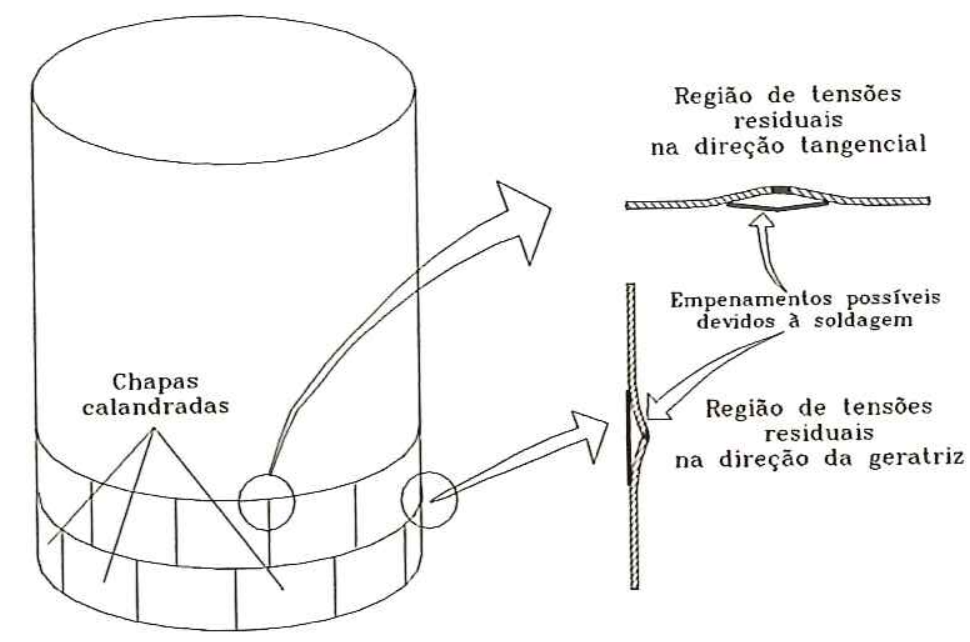

Figura 28 - Disposição das chapas calandradas e regiões de tensões residuais.

Neste capítulo, tratar-se-á primeiro da caracterização do fenômeno de flambagem, com uma classificação dos tipos de flambagem e, em seguida, dos modos axissimétrico e assimétrico de flambagem. Por último, serão mostrados e analisados os casos previstos na norma do ECCS (1988) para a perda de estabilidade do equilíbrio em 1) compressão axial, 2) compressão axial combinada à pressão interna, 3) compressão axial combinada com flexão.

É importante notar que a teoria a ser exposta refere-se à perturbação de posição e não à perturbação de velocidade de deformação da estrutura, o que caracteriza um estudo puramente estático e pode ser justificado pela teoria dos sistemas conservativos.

Para efeito prático, o que interessa determinar é o modo como a estrutura irá flambar e a tensão crítica que define o esgotamento da capacidade portante da estrutura. 


\section{2 - Caracterização do fenômeno.}

Adota-se de início a definição do conceito de flambagem como o fenômeno que ocorre quando a ação atuante na estrutura alcança um valor máximo, o que significa que isto deve resultar em uma não-linearidade na relação entre a solicitação aplicada e os deslocamentos correspondentes.

Pode-se atribuir essa não-linearidade a duas causas distintas. Na primeira, o material que compõe a estrutura pode deixar de apresentar um comportamento linear entre as tensões e as deformações, e a causa é geralmente conhecida como não-linearidade material. $\mathrm{Na}$ segunda causa, o que ocorre são as mudanças na geometria da estrutura provocarem a nãolinearidade, e esse tipo é conhecido como não-linearidade geométrica.

Neste caso de estudo de silos metálicos de paredes delgadas, o que ocorre é a flambagem com o aço ainda no regime elástico, justamente devido à pequena espessura da parede em relação ao raio da estrutura cilíndrica. Vale esclarecer que, até ocorrer a flambagem, a estrutura é admitida no regime linear geométrico, ou seja, pequenos deslocamentos e pequenas deformações. Na realidade, não interessa à engenharia civil que a estrutura de um silo trabalhe em regime de não-linearidade geométrica, sendo bastante uma previsão de início de flambagem com um valor limite, chamado valor crítico de tensão.

Seja um cilindro submetido a compressão axial. O que ocorre primeiro é uma deformação axial puramente de membrana, com deslocamento axial $w_{2}$, com a parede do cilindro permanecendo reta. A um certo nível de solicitação este caminho fundamental alcança um estado crítico de estabilidade. Acima deste nível de solicitação, o estado de equilíbrio não é mais estável, podendo ocorrer, devido a uma pequena perturbação no deslocamento, um súbito movimento lateral (direção radial).

Qualquer estado de estabilidade que ocorra acima da solicitação crítica, ocorrerá em um caminho secundário (ou de pós-flambagem), que intercepta o caminho primário no estado crítico, formado essencialmente de uma deformação lateral $v_{2}$. Este tipo de comportamento é conhecido como bifurcação. 


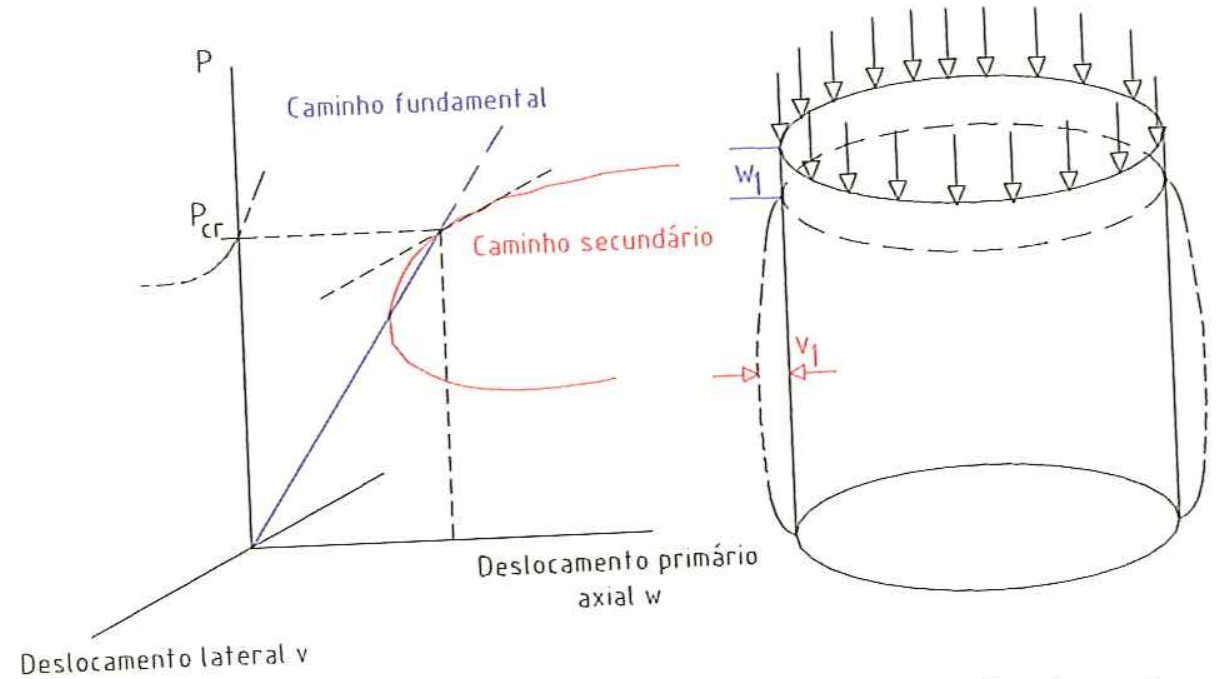

Figura 29 - Caminhos fundamental e secundário de flambagem

Uma classificação prática desse comportamento de bifurcação está ligada à forma que esse caminho secundário pode tomar. Para as estruturas cilíndricas, dentro de modo simples de bifurcação, a forma inicial de deslocamento do caminho secundário pode ser caracterizada em termos de um grau de liberdade, e a flambagem pode ser, como já defínido, ou axissimétrica ou assimétrica.

No próximo tópico será desenvolvida a teoria para o cálculo da tensão crítica de flambagem, em função dos modos predominantes de solicitação: ou axial, ou devido à pressão externa exercida pelo vento.

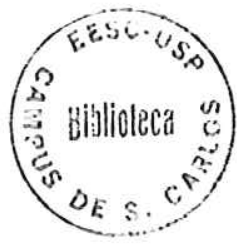




\section{3 - Cálculo da Tensão Crítica de Flambagem.}

A teoria da elasticidade prevê, para uma casca cilíndrica perfeita, isótropa, uniformemente comprimida na direção axial (da geratriz), em um estado puro de tensão de membrana, cujas bordas são indeslocáveis nas direções radial e circunferencial, o início de flambagem simétrica com respeito ao eixo do cilindro [Timoshenko \&Gere (1961)] a uma tensão crítica :

$$
\sigma_{\mathrm{cr}}=\frac{1}{\sqrt{3\left(1-v^{2}\right)}} \cdot \frac{\mathrm{Ee}}{\mathrm{r}} \cong 0,605 \frac{\mathrm{Ee}}{\mathrm{r}}
$$

assumindo o coeficiente de Poisson igual a 0,3.

A determinação desse valor crítico dá-se com a utilização do método da energia. Enquanto a geratriz da casca cilíndrica mantém-se reta, o total da energia de deformação é obtido com a energia de deformação devida à compressão na direção axial. Quando ocorre a flambagem, devem ser consideradas para a energia total de deformação as parcelas de energia na direção tangencial (na circunferência da casca cilíndrica, e com respeito à superfície média) e da energia devida à flexão da casca, que são adicionadas à energia de deformação devida à compressão axial de membrana.

Seja admitida uma combinação arbitrária de pressão lateral externa $p$ e de solicitação axial $N$, que provoque na casca cilíndrica um estado fundamental de esforços solicitantes uniformes de membrana:

$$
\begin{aligned}
& N_{z f}=-\alpha_{z} \sigma_{z} e \\
& N_{\theta f}=-\alpha_{\theta} \sigma_{z} e
\end{aligned}
$$

onde as constantes $\alpha_{z}, \alpha_{\theta}$ são defínidas para o caso de solicitação axial dominante, quando $\alpha_{z}=1:$

$$
\alpha_{\theta}=\frac{N_{\theta f}}{N_{z f}}
$$


e, para o caso de pressão externa dominante, quando $\alpha_{\theta}=1$ :

$$
\alpha_{z}=\frac{N_{z f}}{N_{\theta f}}
$$

Para esses casos, chamados de estados fundamentais $\left(N_{z f}, N_{\theta f}\right)$, estão associadas as deformações uniformes fundamentais:

$$
\begin{aligned}
& \varepsilon_{z f}=\frac{1}{E e}\left(N_{z f}-v N_{\theta f}\right) \\
& \varepsilon_{\theta f}=\frac{1}{E e}\left(N_{\theta f}-v N_{z f}\right)
\end{aligned}
$$

Com respeito a um estado indeformado e sem solicitações externas $(\mathrm{p}=0, \mathrm{~N}=0)$ a mudança na energia potencial total $\left(\mathrm{E}_{\mathrm{pt}}\right)$ para a casca da figura 28 , sob a ação de $\mathrm{p}$ e $\mathrm{N}$ arbitrários e em estado deformado, é (CROLL 1983):

$$
\begin{aligned}
& E_{p t}=\frac{1}{2} \int_{0}^{2 \pi H} \int_{0}^{2}\left(N_{z} \varepsilon_{z}+N_{\theta} \varepsilon_{\theta}+2 N_{z \theta} \varepsilon_{z \theta}\right) r d z d \theta+ \\
& \frac{1}{2} \int_{0}^{2 \pi H} \int_{0}\left(M_{z} \delta_{z}+M_{\theta} \delta_{\theta}+2 M_{z \theta} \delta_{z \theta}\right) r d z d \theta+ \\
& -\left.N W\right|_{0} ^{H}-\int_{0}^{2 \pi H} \int_{0} p v r d z d \theta
\end{aligned}
$$
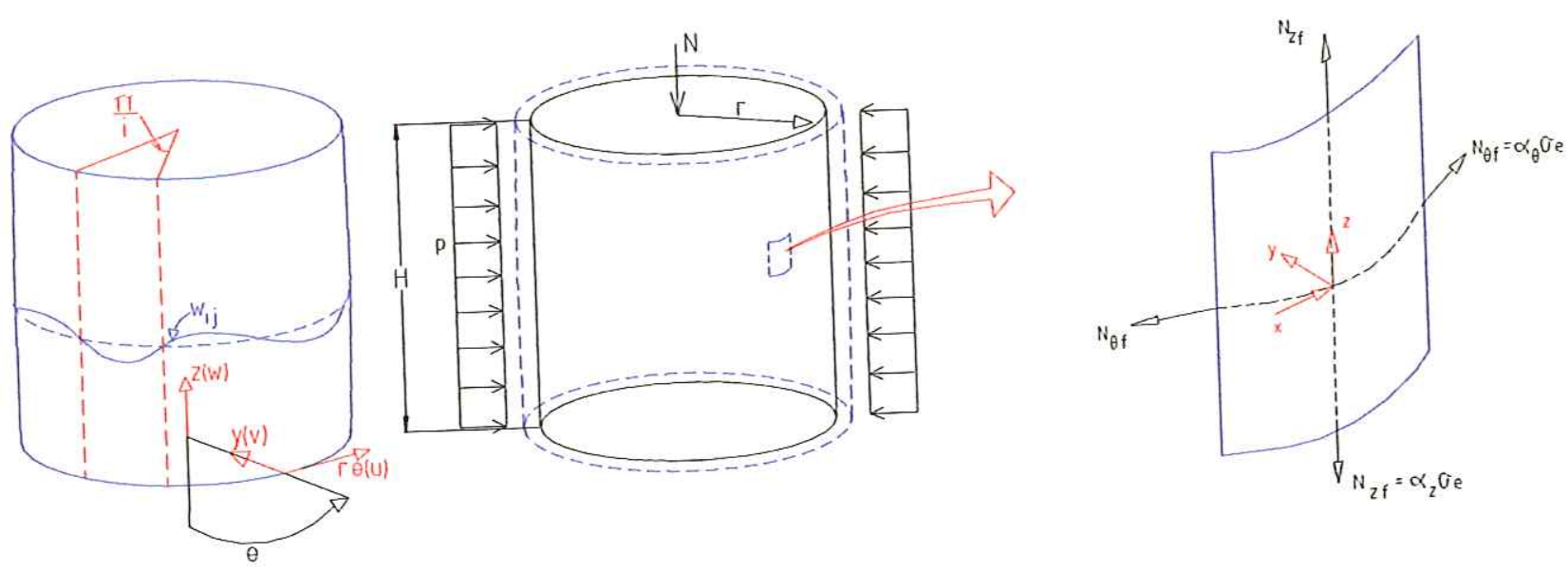

Figura 30 - Estado Fundamental de Deformação 
Na equação 53 as resultantes por unidade de comprimento ( $N$,. M) e suas respectivas deformações $(\varepsilon, \delta)$ e deslocamentos $(U, V, W)$ são medidos em relação à estrutura indeformada. A Ept pode ser reescrita como:

$$
\begin{aligned}
& E_{p t}=\frac{1}{2} \int_{0}^{2 \pi} \int_{0}^{H}\left[N_{z f}+n_{z}\right)\left(\varepsilon_{z f}+\varepsilon_{z}\right)+\left(N_{\theta f}+n_{\theta}\right)\left(\varepsilon_{\theta f}+\varepsilon_{\theta}\right)+ \\
& \left.2\left(N_{\theta f}+n_{z \theta}\right)\left(\varepsilon_{z \theta f}+\varepsilon_{z \theta}\right)\right] r d z d \theta+ \\
& \frac{1}{2} \int_{0}^{2 \pi H} \int_{0}\left[M_{z f}+m_{z}\right)\left(\delta_{z f}+\delta_{z}\right)+\left(M_{\theta f}+m_{\theta}\right)\left(\delta_{\theta f}+\delta_{\theta}\right)+ \\
& \left.2\left(M_{z \theta f}+m_{z \theta}\right)\left(\delta_{z \theta f}+\delta_{z \theta}\right)\right] r d z d \theta+ \\
& -\left.N\left(W_{f}+u\right)\right|_{0} ^{H}-\int_{0}^{2 \pi H} \int_{0}^{2 \pi} p\left(v_{f}+v\right) r d z d \theta
\end{aligned}
$$

A equação 52, quando expandida, pode ser dividida em parcelas tais que:

$$
E_{p t}=E_{p t_{0}}+E_{p t_{1}}+E_{p t_{2}}+\ldots
$$

onde os termos que envolvem potências constantes, lineares, quadráticos, cúbicos, etc., relacionados aos deslocamentos incrementais $(\mathrm{u}, \mathrm{v}, \mathrm{w})$, quando ocorre o estado fundamental de membrana tal que obedeça às eq. $44 \mathrm{e} 45$, são dados por:

$$
\begin{aligned}
E_{p t_{0}}= & \frac{1}{2} \iint\left(N_{z f} \varepsilon_{f}+N_{\theta z} \varepsilon_{\theta f}\right) r d z d \theta-\left.N W_{f}\right|_{0} ^{H}-\iint p v_{f} r d z d \theta \\
E_{p t_{1}}= & \frac{1}{2} \iint\left[\left(N_{z f} \varepsilon_{z}^{\prime}+n_{z}^{\prime} \varepsilon_{z f}\right)+\left(N_{\theta f} \varepsilon_{\theta f}^{\prime}\right)\right] r d z d \theta-\left.P W\right|_{0} ^{H}-\iint p v r d z d \theta \\
E_{p t_{2}}= & \frac{1}{2} \iint\left[n_{z}^{\prime} \varepsilon_{z}^{\prime}+n_{\theta}^{\prime}+2 n_{z}^{\prime} \varepsilon_{z}^{\prime}\right] r d z d \theta+ \\
& \frac{1}{2} \iint\left[m_{z}^{\prime} \delta_{z}^{\prime}+m_{\theta}^{\prime} \delta_{\theta}+2 m_{z \theta}^{\prime} \delta_{z \theta}^{\prime}\right] r d z d \theta+ \\
& \frac{1}{2} \iint\left[\left(N_{z f}^{\prime} \varepsilon_{z}^{\prime \prime}+n_{z}^{\prime \prime} \varepsilon_{z f}\right)+\left(N_{\theta f} \varepsilon_{\theta}^{\prime}+n_{\theta}^{\prime \prime} \varepsilon_{\theta f}\right)\right] r d z d \theta
\end{aligned}
$$


Os termos $E_{p t_{0}}, E_{p t_{1}}, E_{p t_{2}}$ desenvolvidos nas equações $54,55,56$ têm os incrementos lineares dos esforços resultantes (n', m' ), que estão associados às deformações lineares $(\varepsilon ', \delta$ ') definidas como:
$\varepsilon_{z}^{\prime}=\frac{\partial v}{\partial z}$
eq. 57
$\delta_{z}^{\prime}=\frac{\partial^{2} v}{\partial z^{2}}$
eq. 60
$\varepsilon_{\theta}^{\prime}=\frac{1}{r} \frac{\partial \imath}{\partial \theta}-\frac{v}{r}$
eq. 58
$\delta_{\theta}^{\prime}=\frac{1}{r^{2}} \frac{\partial^{2} v}{\partial \theta^{2}}$
eq. 61
$\varepsilon_{z \theta}^{\prime}=\frac{1}{2}\left(\frac{\partial v}{r \partial \theta}+\frac{\partial u}{\partial z}\right)$
$\delta_{z \theta}^{\prime}=\frac{1}{r} \frac{\partial^{2} v}{\partial \theta \partial z}$
eq. 62

e os esforços de membrana n" são aqueles associados às deformações $\varepsilon$ ", dadas por:

$$
\begin{aligned}
& \varepsilon_{z}^{\prime \prime}=\frac{1}{2}\left(\frac{\partial}{\partial z}\right)^{2} \\
& \varepsilon_{\theta}^{\prime \prime}=\frac{1}{2 r^{2}}\left(\frac{\partial}{\partial \theta}\right)^{2}
\end{aligned}
$$

As resultantes lineares dos esforços solicitantes estão relacionadas às deformações lineares com as expressões:

$$
\begin{array}{llll}
n_{z}^{\prime}=K\left(\varepsilon_{z}^{\prime}+v \varepsilon_{\theta}^{\prime}\right) & \text { eq. } 65 & m_{z}^{\prime}=D\left(\delta_{z}^{\prime}+v \delta_{\theta}^{\prime}\right) & \text { eq. } 68 \\
n_{\theta}^{\prime}=K\left(\varepsilon_{\theta}^{\prime}+v \varepsilon_{z}^{\prime}\right) & \text { eq. } 66 & m_{\theta}^{\prime}=D\left(\delta_{\theta}^{\prime}+v \delta_{z}^{\prime}\right) & \text { eq. } 69 \\
n_{z \theta}^{\prime}=K(1-v) \varepsilon_{z \theta}^{\prime} & \text { eq. } 67 & m_{z \theta}^{\prime}=D(1-v) \delta_{z \theta}^{\prime} & \text { eq. } 70
\end{array}
$$

onde $K=\frac{E}{3(1-2 v)}$ e $D=\frac{E e^{3}}{12\left(1-v^{2}\right)}$. As equações relacionadas n $\mathrm{z}_{\mathrm{z}}$ e $\mathrm{n}^{\prime \prime}$ :

$$
n_{z}^{\prime \prime}=K\left(\varepsilon_{z}^{\prime \prime}+\varepsilon_{\theta}^{\prime \prime}\right)
$$




$$
n_{\theta}^{\prime \prime}=K\left(\varepsilon_{\theta}^{\prime \prime}+\varepsilon_{z}^{\prime \prime}\right)
$$

onde $\mathrm{n}_{\mathrm{z}}$ " e $\mathrm{n}_{\theta}$ " são as componentes quadráticas dos esforços solicitantes não-lineares de membrana.

No desenvolvimento da equação 51 o termo $E_{p t_{0}}$ (eq. 54), que é constante em relação aos incrementos de deslocamentos mas variável a cada incremento de solicitações, representa a magnitude de $\mathrm{E}_{\mathrm{pt}}$ para o caminho fundamental $\psi_{1}$ (figura 31):

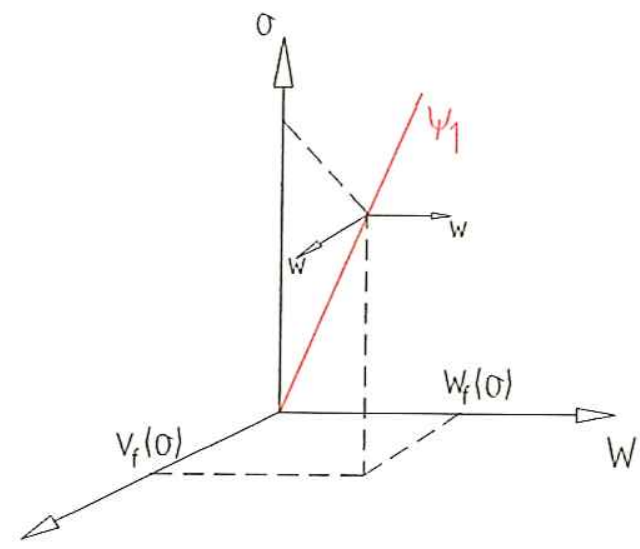

Figura 31 - Caminho fundamental $\psi_{1}$

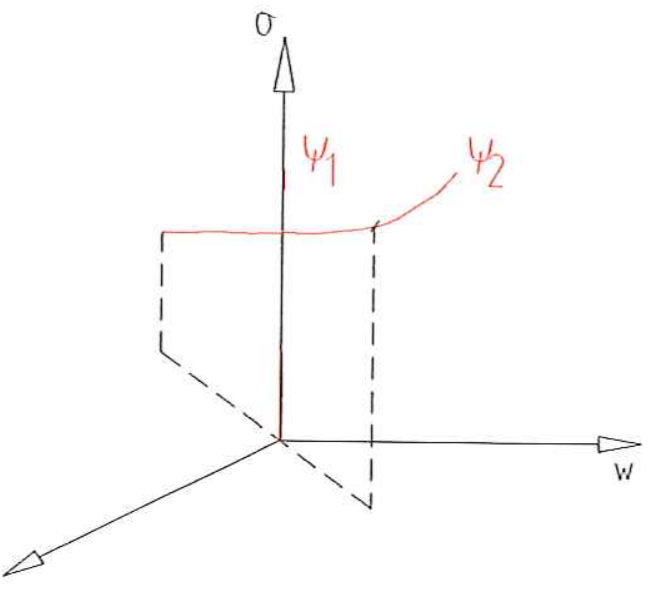

Figura 32 - Caminho secundário $\psi_{2}$

O termo linear $E_{p t_{1}}$, tomado em consideração ao estado de equilíbrio em $\psi_{1}$, deve ser nulo, ou muito próximo de zero, quando os efeitos de flexão são negligenciados nas bordas da casca. Para pequenas perturbações em relação à configuração fundamental é a forma quadrática $E_{p t_{2}}$ que provém as primeiras contribuições significativas. Se o estado de equilíbrio é estável, então $E_{p t_{2}}>0$; se o estado de equilíbrio é instável, haverá pelo menos um modo de deformação em que $E_{p t_{2}}=0$. A situação que corresponde à estabilidade crítica é definida para $E_{p t_{2}}=0$; os modos $\left(\mathrm{u}_{\mathrm{c}}, \mathrm{v}_{\mathrm{c}}, \mathrm{w}_{\mathrm{c}}\right)$ correspondentes são definidos como os modos críticos e a tensão $\sigma_{\mathrm{c}}$ é chamada tensão crítica.

Portanto, o estudo da estabilidade do equilíbrio no caminho fundamental $\psi_{1}$ está todo de acordo com a natureza da forma quadrática $E_{p t_{2}}$. A expressão $E_{p t_{2}}$, dada pela equação 58 , é formada por parcelas distintas, de modo que as energias de deformação 
linear de membrana, $E_{N}$, e de flexão, $E_{p}$, podem ser decompostas em contribuições axial, tangencial, cisalhante (direção v):

$$
\begin{array}{llll}
E_{N}^{z}=\frac{1}{2} \iint n_{z}^{\prime} \varepsilon_{z}^{\prime} r d z d \theta & \text { eq. } 73 & E_{p}^{z}=\frac{1}{2} \iint m_{z}^{\prime} \delta_{z}^{\prime} r d z d \theta & \text { eq. } 76 \\
E_{N}^{\theta}=\frac{1}{2} \iint n_{\theta}^{\prime} \varepsilon_{\theta}^{\prime} r d z d \theta & \text { eq. } 74 & E_{p}^{z}=\frac{1}{2} \iint m_{\theta}^{\prime} \delta_{\theta}^{\prime} r d z d \theta \\
E_{N}^{z \theta}=\frac{1}{2} \iint n_{z \theta}^{\prime} \varepsilon_{z \theta}^{\prime} r d z d \theta & \text { eq. } 75 & E_{p}^{z}=\frac{1}{2} \iint 2 m_{z \theta}^{\prime} \delta_{z \theta}^{\prime} r d z d \theta & \text { eq. } 77
\end{array}
$$

As equações 73 a 78 são os termos lineares da energia de deformação, de forma a distinguir dos termos não-lineares da energia de deformação de membrana $\left(\mathrm{G}_{\mathrm{N}}\right)$ :

$$
\begin{array}{llll}
\bar{G}_{N}^{z}=\frac{1}{2} \iint N_{z f} \varepsilon_{z}^{\prime \prime} r d z d \theta & \text { eq. 79 } & \bar{G}_{N}^{\theta}=\frac{1}{2} \iint N_{\theta f} \varepsilon_{\theta}^{\prime \prime} r d z d \theta & \text { eq. 81 } \\
\overline{\bar{G}}_{N}^{z}=\frac{1}{2} \iint n_{z}^{\prime \prime} \varepsilon_{z f} r d z d \theta & \text { eq. 80 } & \bar{G}_{N}^{\theta}=\frac{1}{2} \iint n_{\theta}^{\prime \prime} \varepsilon_{\theta f} r d z d \theta & \text { eq. 82 }
\end{array}
$$

As equações 81 a 84 advêm da interação entre os esforços solicitantes e as deformações fundamentais $\left(N_{\theta f},{ }_{\mathrm{f}} N_{\theta f}, \varepsilon_{z f}, \varepsilon_{\theta f}\right.$, respectivamente) e as componentes dos incrementos dos esforços, das forças e deformações. As razões pelas quais há a separação nos termos $\bar{U}_{N}^{z}, \overline{\bar{U}}_{N}^{z}, \bar{U}_{\theta}^{z}, \overline{\bar{U}}_{\theta}^{z}$ serão dadas no tópico que trata de flambagem assimétrica para a solicitação axial dominante.

Todo esse desenvolvimento deve conduzir à determinação da tensão crítica de flambagem em uma análise de bifurcação. Seja uma casca cilíndrica com os modos de incremento para os deslocamentos dados por:

$$
\begin{aligned}
& u=u_{i j} \operatorname{sen}(i \theta) \operatorname{sen}\left(\frac{j \pi}{H} z\right) \\
& v=v_{i j} \cos (i \theta) \operatorname{sen}\left(\frac{j \pi}{H} z\right) \\
& w=w_{i j} \cos (i \theta) \cos \left(\frac{j \pi}{H} z\right)
\end{aligned}
$$


Essas equações 85 a 87 satisfazem à clássica condição de vinculação das bordas da casca, ou seja, para $\mathrm{n}_{\mathrm{Z}}=\mathrm{u}=\mathrm{v}=\mathrm{m}_{\mathrm{Z}}=0$ ( as bordas estão com os deslocamentos impedidos). Para que o caminho $\Psi_{2}$ intercepte $\Psi_{1}$ ( figura 30), ou utilizando uma descrição de certo modo enganosa, para que o caminho $\Psi_{2}$ bifurque do caminho $\Psi_{1}$, é necessário que exista um estado de equilíbrio adjacente. Isto ocorre quando $E_{p t_{2}}$ é estacionária com respeito a cada um dos deslocamentos generalizados $\left(\mathrm{u}_{\mathrm{ij}}, \mathrm{v}_{\mathrm{ij}}, \mathrm{w}_{\mathrm{ij}}\right)$.

Fazendo $E_{p t_{2}}$ estacionário com respeito a $\left(\mathrm{u}_{\mathrm{ij}}, \mathrm{v}_{\mathrm{ij}}\right)$ conduz a duas condições que podem ser expressas por (CROLL 1983):

$$
\begin{aligned}
& u_{i j}=\frac{i\left(i^{2}+(2+v) \lambda^{2}\right)}{\left(i^{2}+\lambda^{2}\right)} v_{i j} \\
& w_{i j}=\frac{\lambda\left(i^{2}+v \lambda^{2}\right)}{\left(i^{2}+\lambda^{2}\right)} v_{i j}
\end{aligned}
$$

Estas expressões 88 e 89 relacionam os deslocamentos axial $\left(\mathrm{w}_{\mathrm{ij}}\right)$ e tangencial $\left(\mathrm{u}_{\mathrm{ij}}\right)$ ao deslocamento radial $\left(\mathrm{v}_{\mathrm{ij}}\right)$. Por conseguinte, as contribuições lineares da energia de deformação $\left(E_{N}, E_{p}\right)$ podem ser defínidas como:

$$
\begin{aligned}
& E_{N}^{z}=\frac{\pi K L}{4}\left[\frac{\left(1-v^{2}\right) i^{2} \lambda^{4}\left(i^{2}-v \lambda^{2}\right)}{\left(i^{2}+\lambda^{2}\right)^{4}}\right] v_{i j}^{2} \\
& E_{N}^{\theta}=\frac{\pi K L}{4}\left[\frac{\left(1-v^{2}\right) \lambda^{6}\left(\lambda^{2}-v i^{2}\right)}{\left(i^{2}+\lambda^{2}\right)^{4}}\right] v_{i j}^{2} \\
& E_{N}^{\theta_{z}}=\frac{\pi K L}{4}\left[\frac{2\left(1-v^{2}\right)(1+v) i^{2} \lambda^{6}}{\left(i^{2}+\lambda^{2}\right)^{4}}\right] v_{i j}^{2} \\
& E_{p}^{z}=\frac{\pi K L}{4}\left[\frac{1}{\varphi} \lambda^{2}\left(\lambda^{2}+v i^{2}\right)\right] v_{i j}^{2} \\
& E_{p}^{\theta}=\frac{\pi K L}{4}\left[\frac{1}{\varphi} i^{2}\left(i^{2}+v \lambda^{2}\right)\right] v_{i j}^{2} \\
& E_{p}^{\theta z}=\frac{\pi K L}{4}\left[\frac{2}{\varphi}(1-v) i^{2} \lambda^{2}\right] v_{i j}^{2}
\end{aligned}
$$


e os termos não-lineares da energia de deformação de membrana são:

$$
\begin{aligned}
& \bar{G}_{N}^{z}=\frac{\pi K L}{8}\left[\frac{N_{z f}}{K} \lambda^{2}\right] v_{i j}^{2} \\
& \overline{\bar{G}}_{N}^{z}=\frac{\pi K L}{8}\left[\varepsilon_{z f}\left(\lambda^{2}+v i^{2}\right)\right] v_{i j}^{2} \\
& \bar{G}_{N}^{\theta}=\frac{\pi K L}{8}\left[\frac{N_{\theta f}}{K} i^{2}\right] v_{i j}^{2} \\
& \overline{\bar{G}}_{N}^{\theta}=\frac{\pi K L}{8}\left[\varepsilon_{\theta f}\left(i^{2}+v \lambda^{2}\right)\right] v_{i j}^{2}
\end{aligned}
$$

onde $R=\frac{r}{e}, L=\frac{H}{r}, \lambda=\frac{j \pi}{L}, \varphi=12 R^{2}$.

Então, as energias totais de membrana $\left(E_{N}\right)$ e de flexão $\left(E_{p}\right)$ são dadas por:

$$
\begin{aligned}
& E_{N}=E_{N}^{z}+E_{N}^{\theta}+E_{N}^{z \theta}=\frac{\pi K L}{4}\left[\frac{\left(1-v^{2}\right) \lambda^{4}}{\left(i^{2}+\lambda^{2}\right)}\right] v_{i j}^{2} \\
& E_{p}=E_{p}^{z}+E_{p}^{\theta}+E_{p}^{z \theta}=\frac{\pi K L}{4}\left[\frac{\left(i^{2}+\lambda^{2}\right)^{2}}{\varphi}\right] v_{i j}^{2}
\end{aligned}
$$

e a energia não-linear total de membrana é:

$$
G_{N}=\bar{G}_{N}^{z}+\overline{\bar{G}}_{N}^{z}+\bar{G}_{N}^{\theta}+\overline{\bar{G}}_{N}^{\theta}=\frac{\pi K L}{4}\left[N_{z f} \lambda^{2}+N_{\theta f} i^{2}\right] v_{i j}^{2}
$$

Estas equações 98, 99 e 100, permitem verificar quando $E_{p t_{2}}$ é estacionária, ou seja:

$$
\frac{\partial G_{N}}{\partial v_{i j}}=\frac{\pi K L}{2}\left[\frac{\left(i^{2}+\lambda^{2}\right)}{\varphi}+\frac{\left(1-v^{2}\right) \lambda^{4}}{\left(i^{2}+\lambda^{2}\right)^{2}}+\frac{1}{K}\left(N_{z f} \lambda^{2}+N_{\theta f} i^{2}\right)\right] v_{i j}=0
$$


que, para soluções diferentes da trivial $\left(\mathrm{v}_{\mathrm{ij}} \neq 0\right)$, resulta em:

$$
\frac{\sigma_{c}}{E}=\frac{\left[\frac{\left(i^{2}+\lambda^{2}\right)^{2}}{\varphi}+\frac{\left(1-v^{2}\right) \lambda^{4}}{\left(1+\lambda^{2}\right)^{2}}\right]}{\left(1-v^{2}\right)\left(\alpha_{z} \lambda^{2}+\alpha_{\theta} i^{2}\right)}
$$

\section{4 - Caso Axissimétrico de Flambagem.}

Este caso ocorre para a solicitação axial predominante, quando a equação 102 coincide com a clássica fórmula para o cálculo da tensão de flambagem dada em Timoshenko\&Gere (1961), desde que seja assumido $\mathrm{i}=0$ (indica ausência de ondas na direção circunferencial) e substituindo os parâmetros $\alpha_{\mathrm{Z}}=1$ (fator que, quando unitário, indica solicitação axial predominante), $\alpha_{\theta}=0$ (fator que, quando unitário, indica solicitação de pressão externa predominante), $\lambda=\frac{j \pi}{L}=\frac{j \pi r}{H}, \varphi=12 \frac{r^{2}}{e^{2}}$ :

$\sigma_{c r}=\frac{N_{c r}}{2 \pi r e}=D\left(\frac{j^{2} \pi^{2}}{e H^{2}}+\frac{E}{r^{2} D} \frac{H^{2}}{j^{2} \pi^{2}}\right)$

Considerando-se $\sigma_{c r}$ uma função contínua de $\frac{j \pi}{H}$, a equação 103 atinge um valor de mínimo quando:

$$
\begin{gathered}
\frac{\partial \sigma_{c r}}{\hat{\oint}\left(\frac{j \pi}{H}\right)}=0 \Rightarrow D\left(\frac{2}{e} \frac{j \pi}{H}-\frac{2}{r^{2}} \frac{E}{D} \frac{1}{\frac{j^{3} \pi^{3}}{H^{3}}}\right)=0 \Rightarrow \frac{j \pi}{H}=\sqrt[4]{\frac{E e}{r^{2} D}} \\
\text { Substituindo-se o valor de }\left(\frac{j \pi}{H}\right)^{2}=\sqrt{\frac{E e}{r^{2} D}}=\frac{1}{r e} \sqrt{12\left(1-v^{2}\right)} \text { na equação } 103 \mathrm{e}
\end{gathered}
$$
sendo admitido o valor de $v=0,3$, obtém-se: 


$$
\begin{aligned}
& \sigma_{c r}=D\left(\frac{j^{2} \pi^{2}}{e H^{2}}+\frac{E}{r^{2} D} \frac{H^{2}}{j^{2} \pi^{2}}\right)=D\left(\frac{1}{e} \frac{1}{r e} \sqrt{12\left(1-v^{2}\right)}+\frac{E}{r^{2} \frac{E e^{3}}{12\left(1-v^{2}\right)}} \frac{r e}{\sqrt{12\left(1-v^{2}\right)}}\right) \\
& \sigma_{c r}=\frac{2 E e}{r} \frac{1}{\sqrt{12\left(1-v^{2}\right)}}=\frac{1}{\sqrt{3\left(1-v^{2}\right)}} \cdot \frac{E e}{r} \cong 0,605 \frac{E e}{r}
\end{aligned}
$$

Sendo este valor de $\sigma_{c r}$ o mesmo obtido com a equação 44 (caso de solicitação axial de compressão, flambagem axissimétrica).

Analisando-se essa equação 46 para uma casca cilíndrica metálica, E = 205.000 Mpa e supondo $250<\sigma_{c r}<300 \mathrm{Mpa}$, resulta uma relação $500<\frac{r}{e}<400$. Portanto, uma flambagem axissimétrica ocorre no regime elástico para cascas cilíndricas com paredes muito delgadas, com relação raio/espessura da ordem de 500. 


\section{5 - Caso Assimétrico de Flambagem.}

Este caso pode ocorrer quando uma pressão externa $\mathrm{p}$ for dominante $\left(\alpha_{\theta}=1\right)$, ou seja, quando essa ação provoca os efeitos dominantes que levam à flambagem da casca, caso específico do silo vazio e sob a ação do vento. Nesta situação, os esforços solicitantes fundamentais são:

$$
\begin{aligned}
& N_{\theta f}=-\sigma_{z} e=-p r \\
& N_{z f}=\alpha_{z} N_{\theta}^{F}=-\alpha_{z} \sigma_{z} e=-\alpha_{x} p r
\end{aligned}
$$

A equação 104 passa a ser expressa como:

$$
\frac{\sigma_{c}}{E}=\frac{\left[\frac{\left(i^{2}+\lambda^{2}\right)^{2}}{\varphi}+\frac{\left(1-v^{2}\right) \lambda^{4}}{\left(1+\lambda^{2}\right)^{2}}\right]}{\left(1-v^{2}\right)\left(\alpha_{z} \lambda^{2}+i^{2}\right)}
$$

Para uma casca típica com $\mathrm{L}=\mathrm{H} / \mathrm{r}=2, \mathrm{R}=\mathrm{r} / \mathrm{e}=400$, ev $=0,3$, com uma flambagem caracterizada geometricamente por uma semi-onda na direção axial, ou seja, $j=1$, pode-se relacionar o espectro de tensão crítica .

Para uma variação do número de ondas na direção circunferencial, i, a tensão crítica mínima corresponde ao modo $\mathrm{i}_{\mathrm{CM}}=8,5(\mathrm{CM}=$ crítico mínimo $)$. A localização do número ${ }^{i} \mathrm{CM}$ geralmente é efetuada com um procedimento numérico ou gráfico, como ocorre nas normas inglesas e européias (CROLL 1983).

Entretanto, para muitas cascas calcula-se que o comprimento da semi-onda na circunferência, $l_{\theta}=\frac{\pi r}{i_{C M}}$, que caracteriza o modo mínimo, é bem menor que o comprimento da semi-onda na direção da geratriz, $l_{z}$. Isto significa que $\frac{i_{C M}}{\lambda}=\frac{l_{z}}{l_{\theta}}<1$, ou, mais ainda, que $\left(\frac{i}{\lambda}\right)^{2}<<1$. Admitindo essa hipótese, a equação 109 pode ser aproximada por: 


$$
\frac{\bar{\sigma}_{c}}{E}=\left[\frac{\frac{i^{4}}{\varphi}+\frac{\left(i-v^{2}\right) \lambda^{4}}{i^{4}}}{\left(1-v^{2}\right) i^{2}}\right]
$$

que apresenta um mínimo para:

$$
\bar{i}_{C M}=\left[3 \varphi \lambda^{4}\left(1-v^{2}\right)\right]^{1 / 8}
$$

Pode-se utilizar esse valor de mínimo para i CM na equação 109, para se obter uma resposta aproximada da mínima tensão crítica.

\section{6 - A perda de estabilidade devida à compressão axial.}

Este caso reflete uma grande discrepância entre os valores da força crítica para as estruturas reais e os valores teóricos obtidos com a teoria da estabilidade elástica. As razões são comumente atribuídas às imperfeições na parede da estrutura cilíndrica e às condições de vinculação das bordas. Em suma, assume-se a análise para uma estrutura perfeitamente cilíndrica, sem defeitos, idealizada para um estado puro de tensão de membrana.

A teoria da estabilidade elástica prevê, para uma casca cilíndrica perfeita, uniformemente comprimida na direção axial, em um estado puro de tensão de membrana, cujas bordas são indeslocáveis nas direções radial e circunferencial, o início de flambagem simétrica com respeito ao eixo do cilindro [Timoshenko \&Gere (1961)] a uma tensão de:

$$
\sigma_{\mathrm{cr}}=\frac{1}{\sqrt{3\left(1-v^{2}\right)}} \cdot \frac{\mathrm{Ee}}{\mathrm{r}} \cong 0,605 \frac{\mathrm{Ee}}{\mathrm{r}}
$$

assumindo o coeficiente de Poisson igual a 0,3.

A tensão crítica de flambagem, $\sigma_{\mathrm{cr}}$, é uma expressão clássica da teoria da elasticidade considerada função contínua $\operatorname{de} m \pi / \mathbf{H}$, com $\mathrm{m}$ representado o número de ondas da configuração de flambagem elástica da casca cilíndrica de altura $\mathrm{H}$. 
Na realidade, verificou-se experimentalmente [Brush \& Almroth (1975)] que, para um conjunto de cilindros axialmente comprimidos com relações raio/espessura entre $100 \mathrm{e}$ 500, a tensão crítica de flambagem fícou com valores de 30 a $40 \%$ do valor da expressão clássica de tensão crítica (equação 46).

Salienta-se que nesse tipo de análise o cilindro é comprimido axialmente quando está vazio, ou seja, sem pressão interna e sem pressão externa.

Para a análise da ação do vento nos silos e reservatórios vazios, devido à grande sensibilidade da força crítica às imperfeições, será utilizada a expressão da tensão crítica de flambagem, $\sigma_{\mathfrak{U}}$, das recomendações européias para construção metálica do ECCS (1988) para a flambagem de cascas de aço, descritas nas equações 47 a 60 :

$$
\begin{aligned}
& \sigma_{\mathrm{f}, \mathrm{u}}=0,75 . \alpha \cdot \sigma_{\mathrm{cr}} \\
& \text { se } \\
& \alpha . \sigma_{\mathrm{er}} \leq \frac{1}{2} \mathrm{f}_{\mathrm{y}} \\
& \sigma_{f, u}=f_{y}\left[1-0,4123\left(\frac{f_{y}}{\alpha \sigma_{c r}}\right)^{0,6}\right] \\
& \text { se } \quad \alpha . \sigma_{\mathrm{cr}} \geq \frac{1}{2} \mathrm{f}_{\mathrm{y}} \\
& \alpha_{0}=\frac{0,83}{\sqrt{1+0,01 . \mathrm{r} / \mathrm{e}}} \ldots \ldots \ldots \ldots \text { para........ } \mathrm{r} / \mathrm{e}<212 \\
& \alpha_{0}=\frac{0,70}{\sqrt{0,1+0,01 . \mathrm{r} / \mathrm{e}}} \ldots \ldots \ldots \ldots \text { para........ } \mathrm{r} / \mathrm{e}>212
\end{aligned}
$$

Deve-se considerar o caso em que as imperfeições $\bar{u}$ sejam menores que $0,01 \ell_{\mathrm{r}}, l_{\mathrm{r}}=$ 4. $\sqrt{r \cdot e}$, ao longo da altura, $\mathrm{e} \ell_{\mathrm{r}}=(25 . \mathrm{e})$ na circunferência, caso em que valem as equações 47 e 48. O valor de $\ell_{\mathrm{r}}$ não deve exceder $95 \%$ da distância entre os cordões de solda (cordões meridionais - ao longo da altura - ou os cordões circunferenciais. Se $\bar{u} / \ell_{r}$ for igual a 0,02 , os valores de $\alpha$ são reduzidos à metade. Se $\bar{u} / \ell_{r}$ estiver entre 0,01 e 0,02 , pode-se fazer uma interpolação linear entre os valores de $\alpha$ e de $\alpha / 2$. O comprimento $l_{\text {r }}$ está relacionado ao tamanho potencial das ondas de flambagem. (figura 54 - adaptada do ECCS (1988)). 


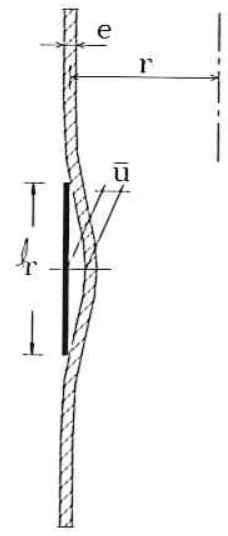

a)

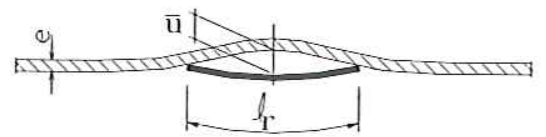

b)

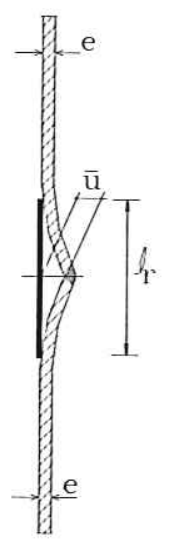

c)

Figura 33 - Medidas de imperfeições.

\section{7 - A perda de estabilidade devida à compressão axial combinada com pressão interna.}

Este caso pode ser aplicado à condição do silo estar preenchido. A pressão interna devida ao material é levada em consideração, sendo consequiência direta o aumento da resistência da estrutura à flambagem por compressão axial.

Será utilizada a expressão das recomendações européias com relação à flambagem das cascas de aço [ECCS (1988)], para uma combinação de compressão axial e pressão interna. Desta forma, a resistência a ser verificada para a estrutura é (fórmula já adaptada às coordenadas adotadas, ou seja, $\mathbf{z}$ na direção da altura do silo, $\mathbf{x}$ tangencial, e $\mathbf{y}$ radial ):

$$
\sigma_{e f, d}=\sqrt{\sigma_{z d}^{2}+\sigma_{x d}^{2}-\sigma_{x d} \sigma_{z d}} \leq \sigma_{e f, u}
$$

Com $\sigma_{\mathrm{ef}, \mathrm{u}}$ definida por:

$$
\sigma_{e f, u}=\sqrt{\sigma_{z u}^{2}+\sigma_{x u}^{2}+\sigma_{z u} \sigma_{x u}}
$$

Que é calculado com:

$$
\frac{\sigma_{\text {ef,u }}}{f_{y}}=\frac{0,75}{\bar{\lambda}_{p}^{2}}
$$

se

$\bar{\lambda}_{p} \geq \sqrt{2}$

eq. 117 


$$
\begin{aligned}
& \frac{\sigma_{e f, u}}{f_{y}}=1-0,4123 \cdot \bar{\lambda}_{p}^{1,2} \\
& \bar{\lambda}_{p}=\sqrt{\frac{\beta \cdot f_{y}}{\alpha_{p} \cdot \sigma_{c r}}} \\
& \alpha_{p}=\alpha_{0}+\left(1-\alpha_{0}\right) \frac{\rho}{\rho+0,007} \\
& \rho=p_{c} \frac{r \sqrt{r}}{E e \sqrt{e}}
\end{aligned}
$$

se

$\bar{\lambda}_{\mathrm{p}} \leq \sqrt{2}$

eq. 118

eq. 119

eq. 120

$\beta$ : é a razão entre a tensão meridional de membrana $\sigma_{\mathrm{zu}}$ e a tensão efetiva $\sigma_{\mathrm{ef}, \mathrm{u}}$ até o início da flambagem:

$$
\beta=\frac{\sigma_{z d}}{\sigma_{e f, d}}
$$

Para este cálculo segundo o ECCS a tensão compressiva de membrana $\sigma_{\mathrm{Zu}}$ deve ser considerada positiva e a tensão tangencial de tração $\sigma_{\mathrm{Xu}}=-\mathrm{p}_{\mathrm{c}} \cdot \mathrm{r} / \mathrm{e}$ como um valor negativo.

Adota-se um valor médio uniforme que seja possível admitir para a pressão lateral interna do silo, $p_{c}$, baseada na equação 31 .

\section{8 - A perda de estabilidade devida à compressão axial combinada com flexão.}

As recomendações do ECCS representam o cálculo de compressão axial combinada com flexão admitindo:

$$
\begin{aligned}
& \alpha=\frac{\alpha_{0}+\alpha_{b} \sigma_{b}}{\sigma_{0}+\sigma_{b}} \\
& \alpha_{b}=0,1887+0,8113 . \alpha_{0} \quad \text { (experimental) }
\end{aligned}
$$


$\alpha_{0}$ : dado pelas equações 113 e 114 .

$\sigma_{0}$ : tensão uniforme de compressão devida à força axial de projeto.

$\sigma_{\mathrm{b}}$ : tensão máxima de compressão devida ao momento de projeto.

Obtido o valor de $\alpha$, é feita a aplicação nas equações 111 ou 112 e verificada a tensão crítica de flambagem.

A tensão $\sigma_{b}$ é obtida para a solicitação do vento quando o silo está vazio, uma vez que o momento de projeto é calculado para a ação do vento. 


\section{Capítulo 4 - Análise Numérica de Cascas Cilíndricas.}

O objetivo da análise numérica é avaliar a distribuição dos esforços e deslocamentos, oriundos das ações permanentes e variáveis, com ênfase nos efeitos devidos ao material armazenado e ao vento, em regime linear de deformações.

Essa ênfase destina-se ao estudo da instabilidade das chapas metálicas do corpo do reservatório em duas situações distintas: 1) quando o reservatório encontra-se vazio e está solicitado pelo vento; 2) quando do fluxo de carga ou descarga do material ensilado.

A análise numérica encontra-se estabelecida para duas categorias de produtos a serem ensilados: 1) os que não provocam atrito nas paredes; 2) os que provocam atrito nas paredes. Também considera-se na análise a variação de espessura das chapas do costado do silo ao longo da altura.

Uma consequiência direta dessa análise numérica está no exemplo de silo metálico para milho, no capítulo 5 .

\section{1 - O Esquema Estático dos Reservatórios.}

Os reservatórios cilíndricos são formados por chapas de aço soldadas entre si e geometricamente definidos pelo diâmetro (D), altura (H) e espessura do corpo (e). O corpo do reservatório é soldado ao fundo, e a tampa pode ser ou inteiramente soldada ou apenas fixada em determinados pontos do topo do reservatório.

As paredes do reservatório são vinculadas ao fundo considerando restrições aos deslocamentos nas direções $x, y, z$, e restrições aos giros em torno dos eixos $x, y, z$. 

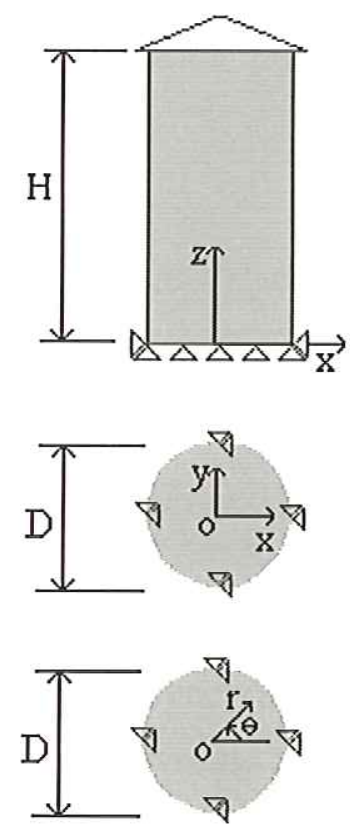

$\mathrm{H}=$ altura do reservatório

$\triangleleft=$ deslocamento impedido na direção $\mathrm{x}$

$\Delta=$ deslocamento impedido na direção $z$

oxyz $=$ sistema de coordenadas global cartesiano

$\mathrm{D}=$ diâmetro do reservatório

$\triangleleft=$ deslocamento impedido na direção $\mathrm{x}$

$\nabla=$ deslocamento impedido na direção y

Giro, em cada eixo, segue a regra da mão direita

or $\theta z=$ sistema de coordenadas cilíndrico

Figura 34 - Esquema Estático dos Reservatórios

\section{2 - Os Modelos Geométricos dos Reservatórios}

O critério para se determinar a relação entre as dimensões do modelo é o volume do reservatório, mantido constante. Conseqüentemente, para um dado volume $V_{0}$ e consideradas as relações altura/diâmetro (H/D) entre 1 e 5, ficam definidas as dimensões das alturas e dos diâmetros para os reservatórios de mesmo volume com H/D $=1$ e H/D $=5$. Também estão calculadas as áreas laterais respectivas a cada um dos modelos.

As dimensões dos diâmetros, das alturas, as proporções entre as mesmas, e os valores das áreas laterais estão na tabela 2 e na figura 36 para os modelos que serão analisados numericamente $(H / D=1$ e $H / D=5)$ e os modelos intermediários $(H / D=2,3,4)$ : 
Definidos: $\quad \mathrm{H}=$ altura do cilindro $\quad \mathrm{D}=$ diâmetro $\quad \mathrm{V}=$ volume $=\pi \frac{D^{2}}{4} H$ Para um dado volume Vo estabelecer uma função $f(y)$, com $y=H / D$.

$V=V_{0} \Leftrightarrow V_{0}=c t e$

$V_{0}=\pi \frac{D^{3}}{4}\left(\frac{H}{D}\right) \Rightarrow D^{3}=\frac{4}{\pi} V_{0} \frac{1}{(H / D)}$

$y=H / D \Rightarrow D^{3}=\frac{4}{\pi} V_{0} \frac{1}{y} \Leftrightarrow D>0$

$\therefore D=K \frac{1}{\sqrt[3]{y}} \Leftrightarrow K=\sqrt[3]{\frac{4}{\pi} V_{0}}$

ou

$f(y)=K \frac{1}{\sqrt[3]{y}} \Leftrightarrow f(y)=D$

\begin{tabular}{|c|c|c|c|}
\hline \multicolumn{4}{|c|}{ TABELA 2 - Relações Altura/Diâmetro e } \\
Área Lateral para um Volume Constante. \\
\hline $\begin{array}{c}\mathrm{y}= \\
\text { H/D }\end{array}$ & D & H & Área lateral \\
\hline 1 & 1 & 1 & 3,142 \\
\hline 2 & 0,794 & 1,588 & 3,961 \\
\hline 3 & 0,693 & 2,079 & 4,526 \\
\hline 4 & 0,630 & 2,520 & 4,988 \\
\hline 5 & 0,585 & 2,925 & 5,377 \\
\hline
\end{tabular}
Área Lateral para um Volume Constante.
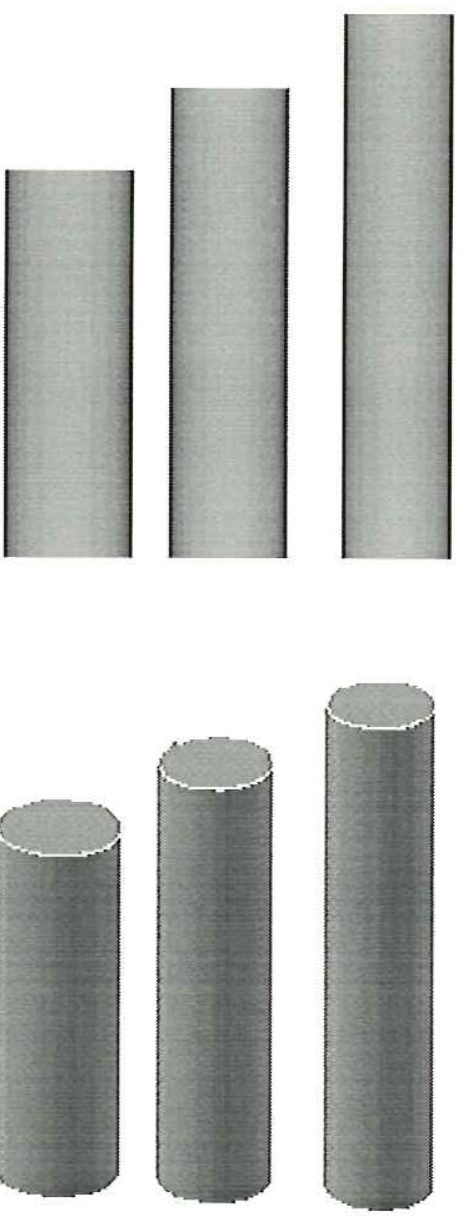

Figura 35 - Modelos Geométricos de Reservatórios Cilíndricos 


\section{3 - As Características do Aço Empregado.}

O aço empregado está caracterizado por um módulo de elasticidade (E) igual a 205.000 MPa, coeficiente de Poisson $(\mu)$ igual a 0,3 , densidade $(\gamma)$ de $77.000 \mathrm{~N} / \mathrm{m}^{3}$, tensão de escoamento $f_{y}=250 \mathrm{MPa}$, e tensão última $f_{\mathrm{u}}=400 \mathrm{MPa}$.

\section{4 - Considerações acerca das Espessuras das Chapas.}

As espessuras das chapas do corpo do reservatório são consideradas em dois casos: 1) constantes ao longo da altura do reservatório; 2) variáveis ao longo da altura do reservatório.
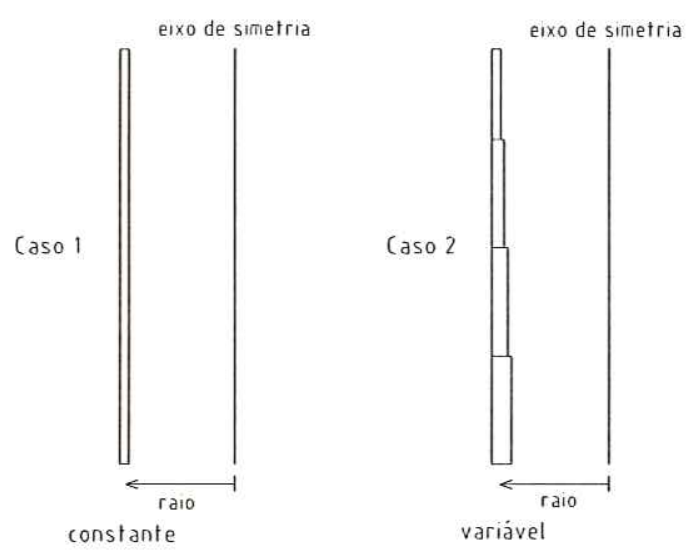

Figura 36 - Variação das Chapas ao Longo da Altura

Como estimativa das espessuras das chapas foi utilizada a expressão de cálculo das tensões anulares de membranas:

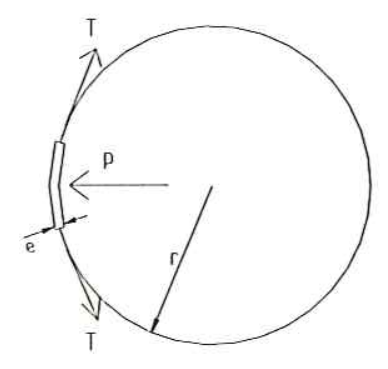

$$
\begin{aligned}
& \mathrm{e}=\text { espessura do elemento } \\
& \mathrm{p}=\text { pressão na face do elemento } \\
& \mathrm{r}=\text { raio da seção transversal } \\
& \mathrm{T}=\text { componente tangencial }
\end{aligned}
$$

Figura 37 - Elemento sob Força Tangencial 
Considerando-se o equilíbrio do elemento de membrana na figura 38 , o valor da tensão de membrana anular que atua no elemento é:

$$
\sigma=\frac{\mathrm{p} \cdot \mathrm{r}}{\mathrm{e}}
$$

\section{5 - Os Casos em Análise}

Considerando a existência ou não de atrito devido ao material armazenado, a condição de o reservatório estar vazio e as condições acerca das espessuras das chapas dos corpos dos reservatórios, resultam, pela combinação das condições anteriores, 6 casos de análise:

r

CASO 1 ) Reservatório com Líquido e Espessura da Parede Constante ao Longo da Altura.
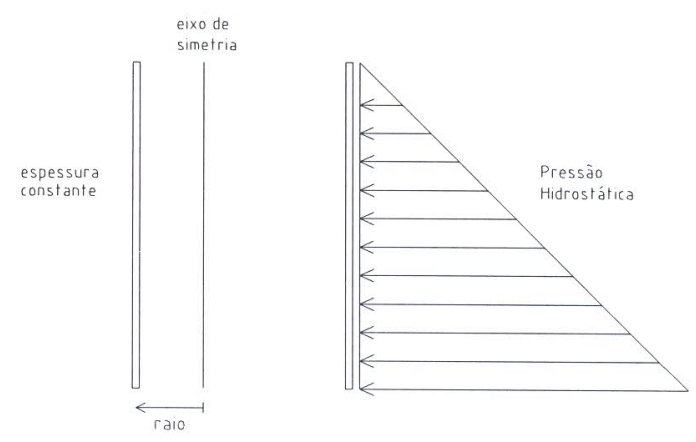

Figura 38 - Caso 1 - Sem atrito: Parede de Espessura Constante

CASO 2) Reservatório com Líquido e Espessura da Parede Variável ao Longo da Altura.
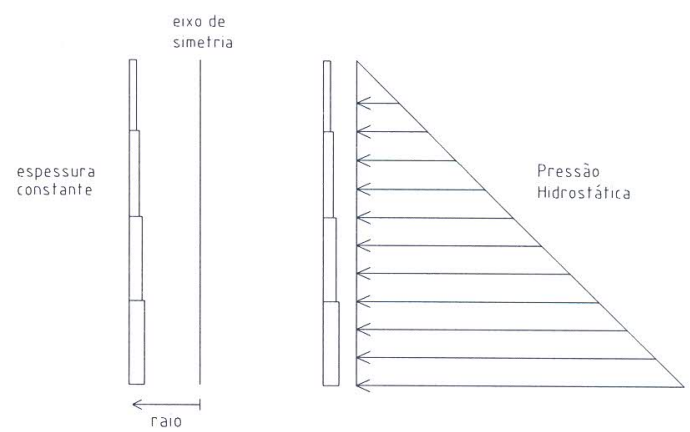

Figura 39 - CASO 2 - Sem atrito: Parede de Espessura Variável 
Caso 3) Reservatório com Material Granular ou Pulverulento e Espessura da Parede Constante ao Longo da Altura.
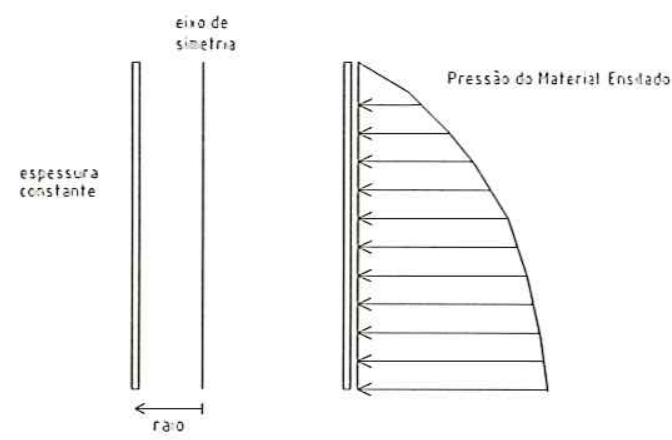

Figura 40 - CASO 3 - Com atrito: Parede de Espessura Constante

CASO 4) Reservatório com Material Granular ou Pulverulento e Espessura da Parede Variável ao Longo da Altura.
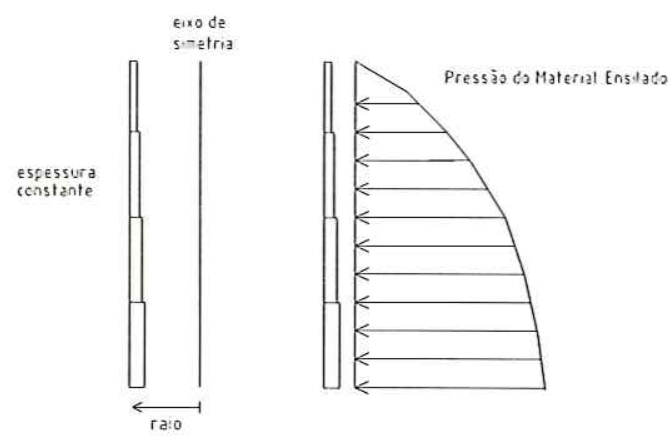

Figura 41 - CASO 4 - Com atrito: Parede de Espessura Variável

CASO 5) Reservatório Vazio e Espessura da Parede Constante ao Longo da Altura.

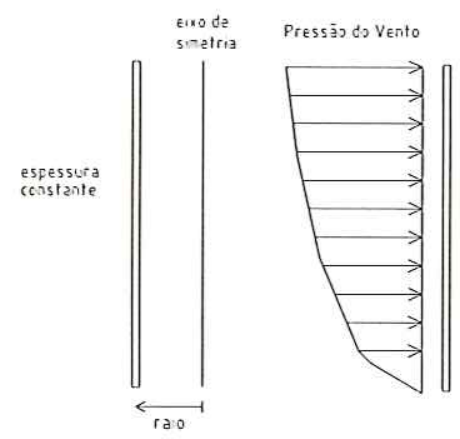

Figura 42 - CASO 5: Reservatório Vazio com Parede de Espessura Constante 
CASO 6) Reservatório Vazio e Espessura da Parede Variável ao Longo da Altura.

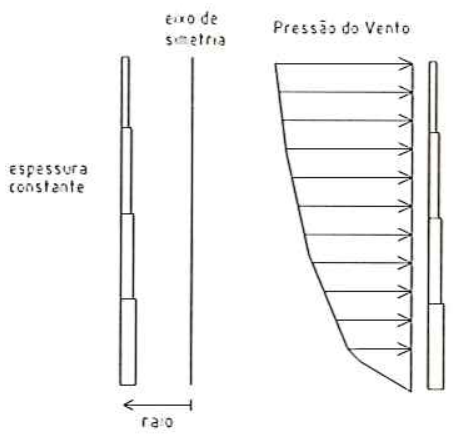

Figura 43 - CASO 6: Reservatório Vazio com Parede de Espessura Variável

\section{6 - As Ações Consideradas}

As ações estão combinadas de acordo com a NBR8681 (1986), sendo destacadas as ações do vento de acordo com a NBR6123 (1987) e a do material armazenado de acordo com a norma DIN1055-6 (1986).

Como ação permanente está considerado o peso próprio da estrutura básica, ou seja, tampa, corpo e fundo do reservatório.

As ações variáveis são as do vento e a do material armazenado.

\section{7 - Combinação de Ações}

As combinações pesquisadas que são aplicadas aos casos 1 a 6 (há mais de um caso que é analisado com a mesma combinação de ações) estão definidas como:

1 - Combinação normal C1 : ação permanente (peso próprio - p.p.), ação variável principal (vento) - aplicada aos casos 5 e 6 ;

2 - Combinação normal C2 : ação permanente (p.p.), ação variável principal(líquido), ação variável secundária ( vento ) - aplicada aos casos 1 e 2;

3 - Combinação normal C3 : ação permanente (p.p.), ação variável principal (sólido), ação variável secundária (vento) - aplicada aos casos 3 e 4; 
Sendo aplicados em cada caso os coeficientes definidos pela NBR8681 - Ações e segurança nas estruturas (1986), resultam as expressões:

$$
\begin{aligned}
& \text { C1 }=1,0 \text { p.p. }+1,4 \text { vento } \\
& \text { C2 }=1,3 p . p .+1,4 \text { líquido }+1,4.0,6 \cdot v e n t o \\
& \text { C3 }=1,3 \text { p.p. }+1,4 \text { sólido }+1,4.0,6 . v e n t o
\end{aligned}
$$

Com as fórmulas descritas no capítulo 2 são determinados os valores das ações empregadas na análise numérica dos modelos 1 e 5 . Os valores estão nas tabelas 4 e 5 para o modelo 1 e nas tabelas 6 e 7 para o modelo 5 . 
Esquema dos valores das ações calculadas para o Modelo $1(10,0$ x 10,0 m)
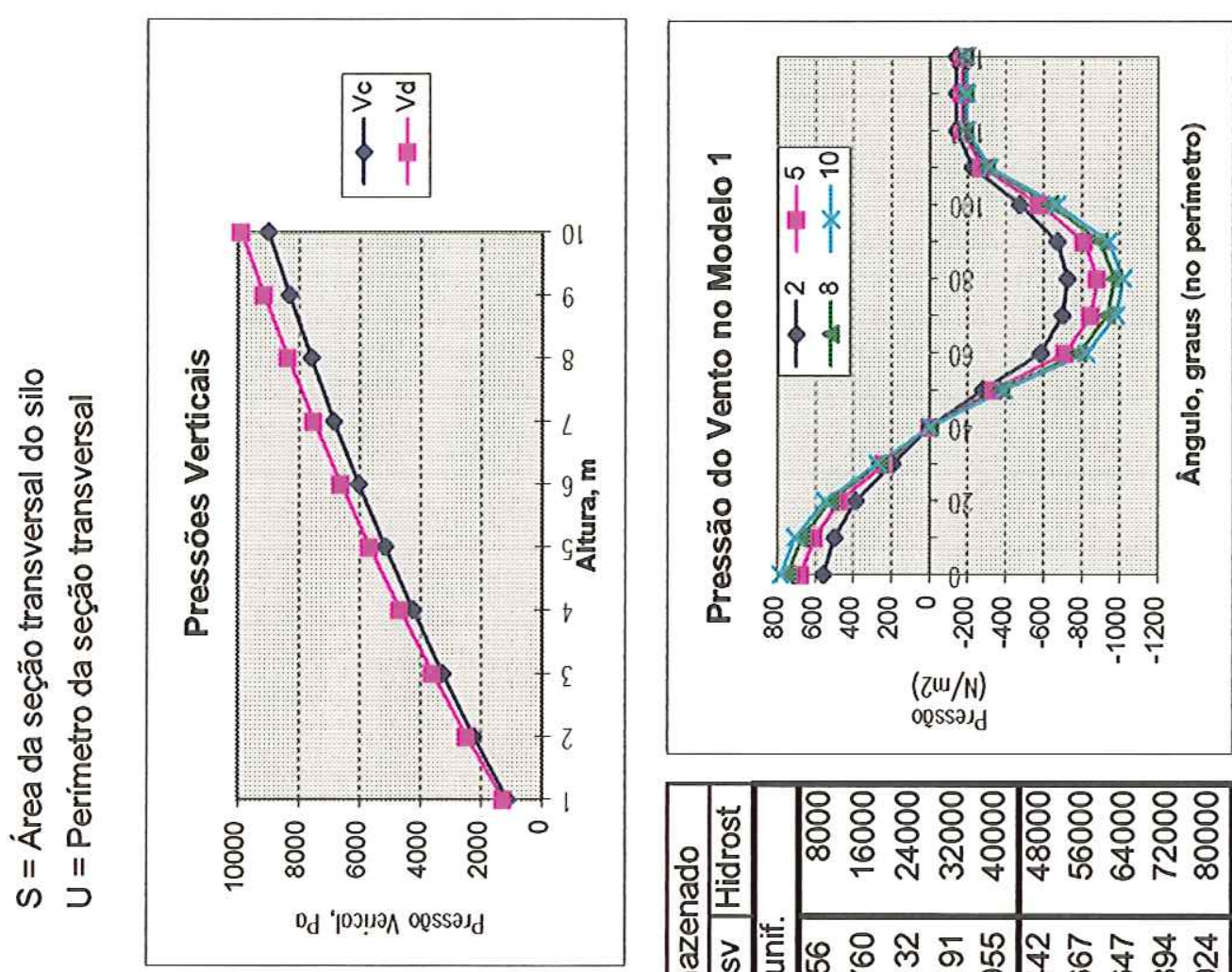

음

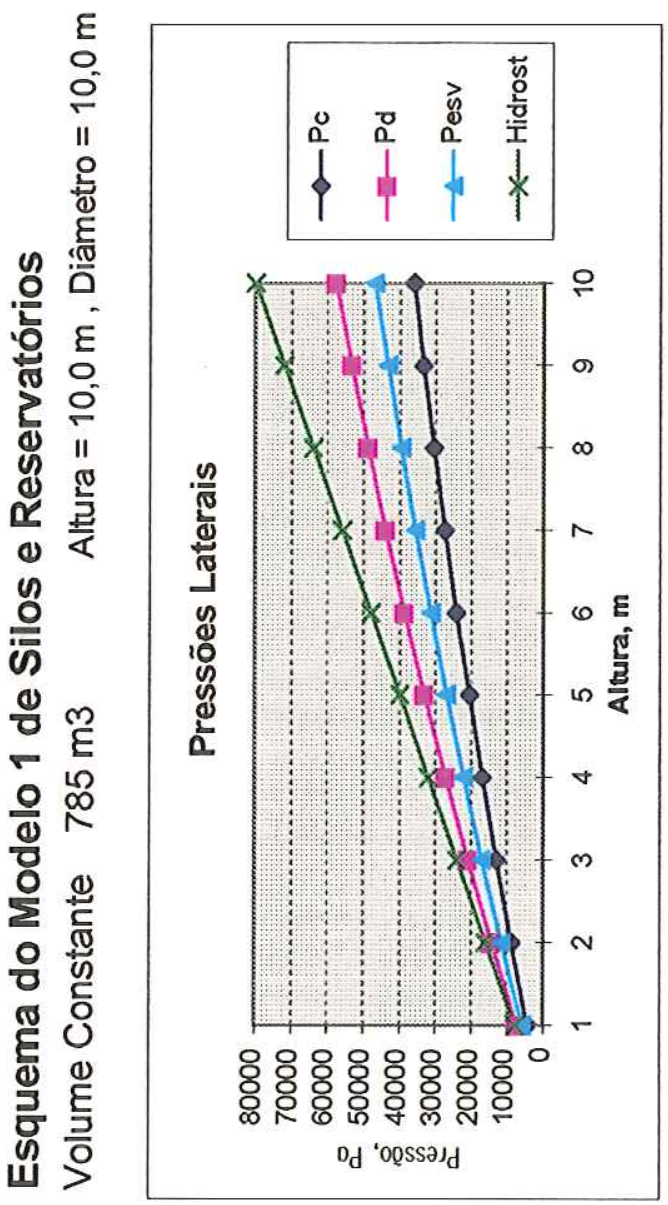

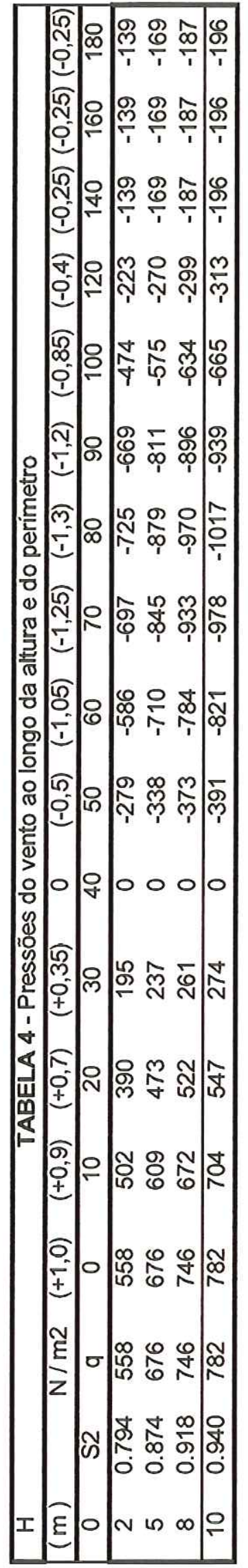


Esquema dos valores das ações calculadas para o Modelo $5(6,0 \times 30,0 \mathrm{~m})$
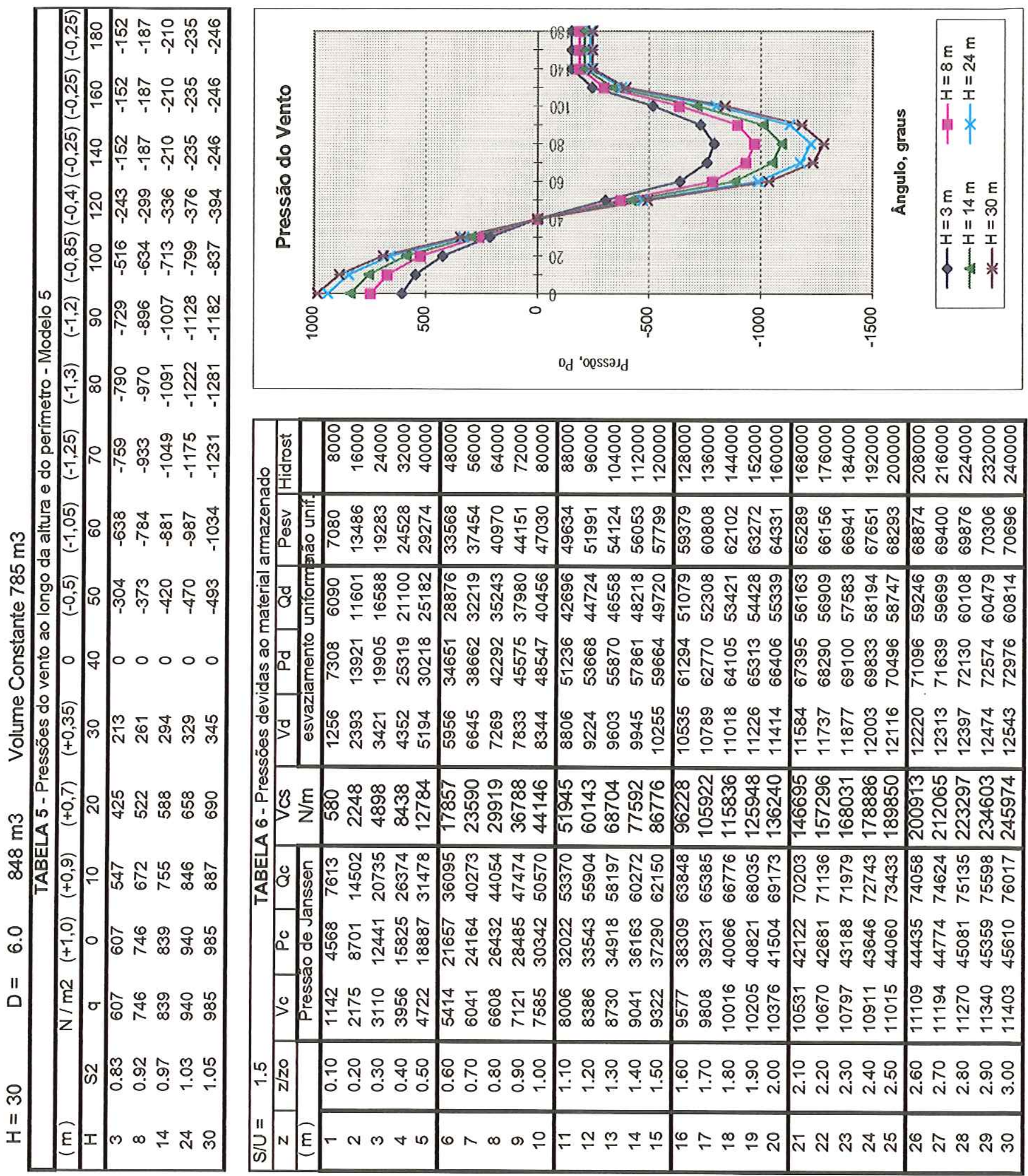

\begin{tabular}{|c|c|c|c|c|c|c|c|}
\hline & 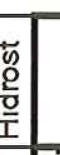 & 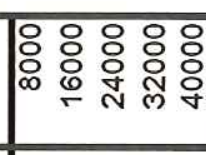 & 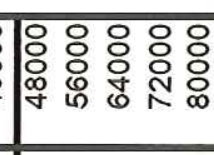 & $\begin{array}{l}0.0 \\
0 \\
0\end{array}$ & 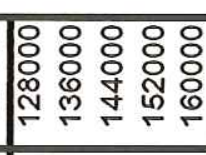 & 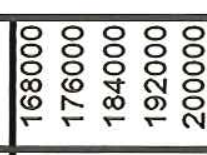 & 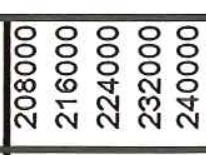 \\
\hline & & 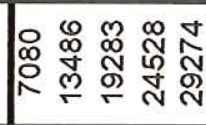 & 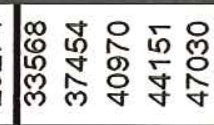 & $\mid \begin{array}{l}4 \\
0 \\
0 \\
0\end{array}$ & 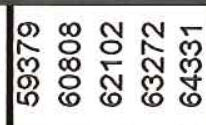 & 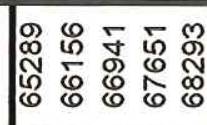 & 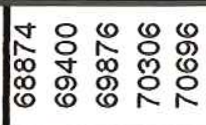 \\
\hline & & 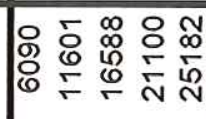 & 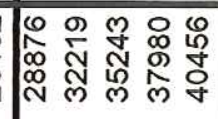 & 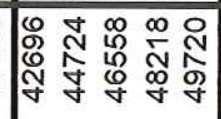 & 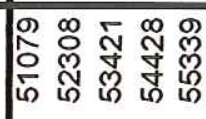 & 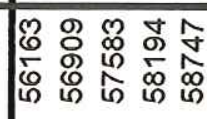 & 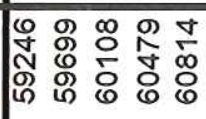 \\
\hline & & 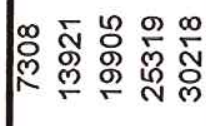 & 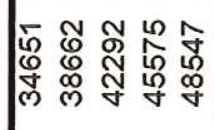 & 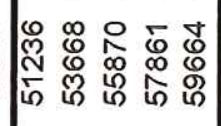 & 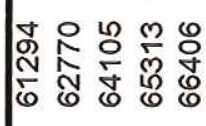 & 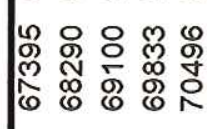 & 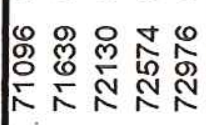 \\
\hline & 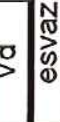 & 兽 & 象 & ஜ & 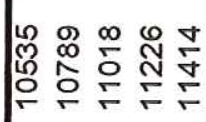 & 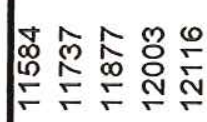 & 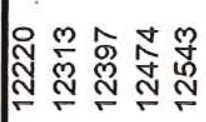 \\
\hline & & 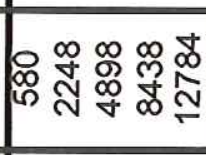 & 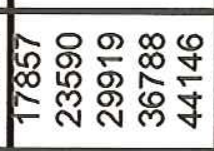 & $\begin{array}{l}\text { W } \\
\text { W } \\
\text { O } \\
\text { in } \\
\text { in } \\
\end{array}$ & 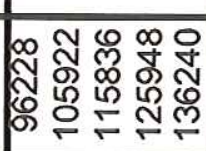 & ஜू & 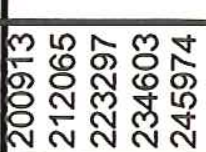 \\
\hline & & 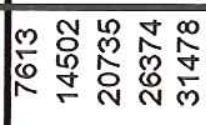 & 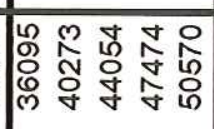 & 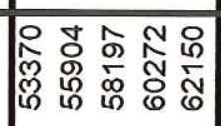 & 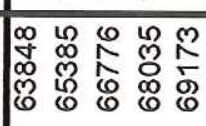 & 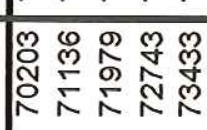 & 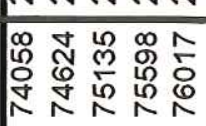 \\
\hline & $\overbrace{0}^{\pi}$ & 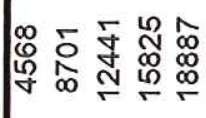 & 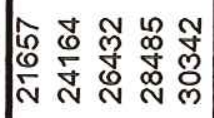 & 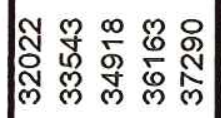 & 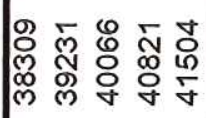 & 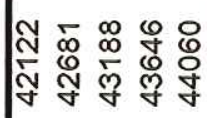 & 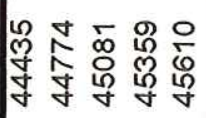 \\
\hline & 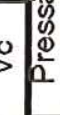 & 군 & 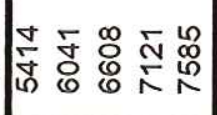 & $\mid$ & 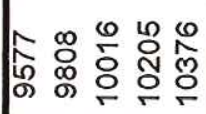 & 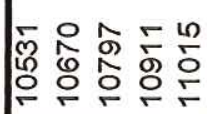 & 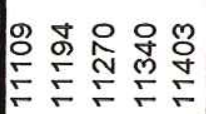 \\
\hline & N & 은 옹요 & 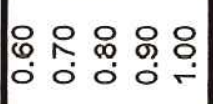 & 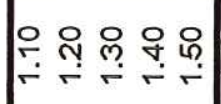 & ن & 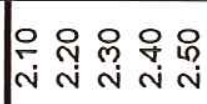 & 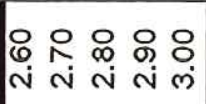 \\
\hline & $\mathrm{N}$ & & & $E$ ? & 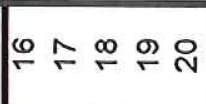 & 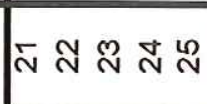 & $\stackrel{N}{N}$ \\
\hline
\end{tabular}


Esquema das ações para o Modelo 5 - Gráficos das Ações devidas ao material granular ensilado, correspondentes à tabela 7 .
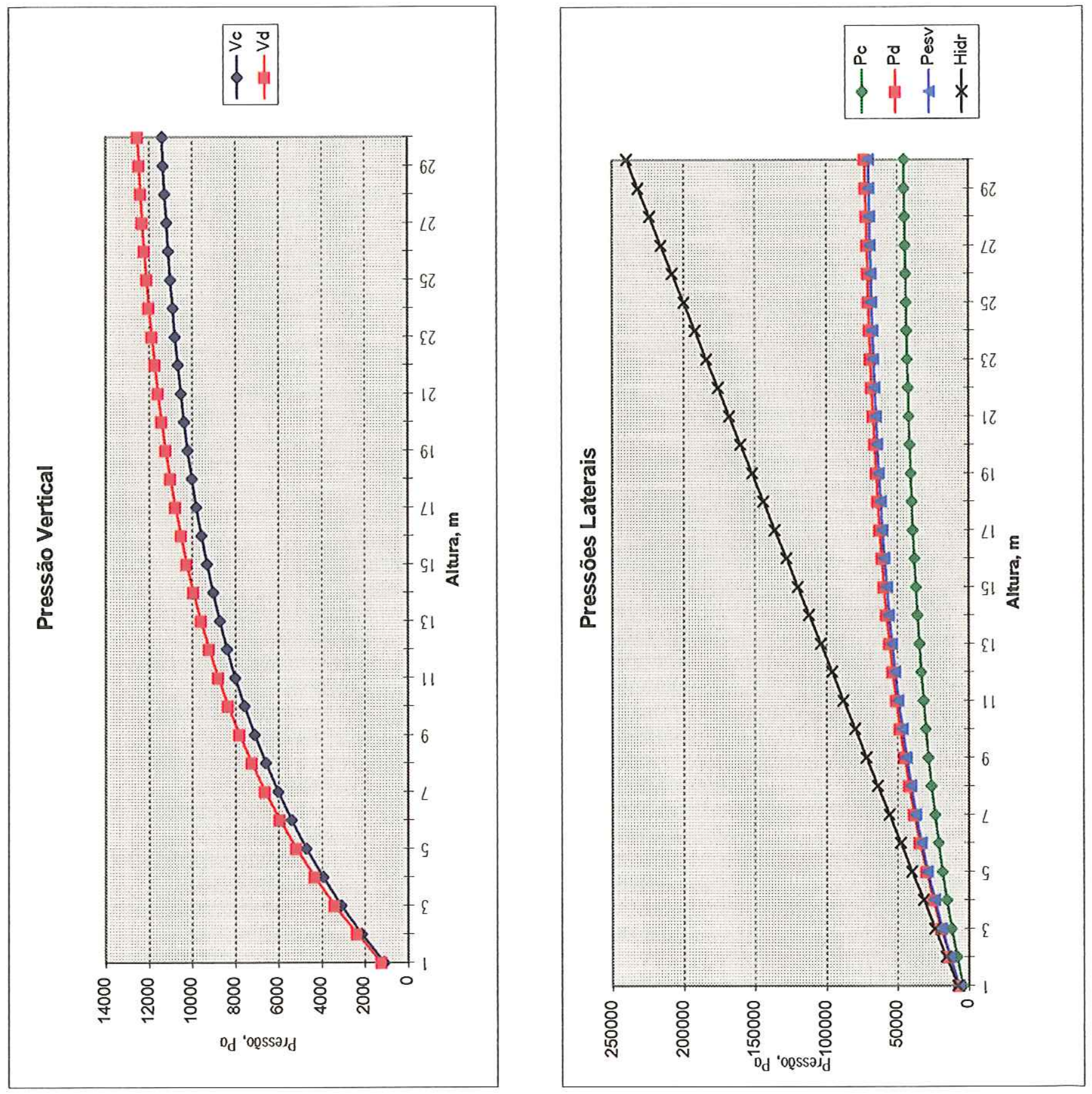
Tabela 7 - Valores para a Força de atritopor unidade de comprimento, ao longo da geratriz do silo, correspondentes aos Modelos 1 e 5.

\begin{tabular}{|c|c|c|c|c|c|}
\hline $\begin{array}{c}\text { Cota } \\
\text { z }\end{array}$ & $\begin{array}{c}\text { Modelo } 1 \\
D=10, H=10 \\
\text { Vcs } 1\end{array}$ & Vcs & $\begin{array}{c}\text { Cota } \\
\text { z }\end{array}$ & $\begin{array}{c}\text { Modelo } 5 \\
D=6, H=30 \\
\text { Vcs } 5\end{array}$ & Vcs \\
\hline 0.25 & 37 & 37 & 0.25 & 37 & 37 \\
\hline 0.5 & 149 & 111 & 0.50 & 148 & 110 \\
\hline 0.75 & 332 & 184 & 0.75 & 329 & 182 \\
\hline 1 & 588 & 256 & 1.00 & 580 & 251 \\
\hline 1.5 & 1310 & 722 & 1.50 & 1285 & 704 \\
\hline 2 & 2307 & 996 & 2.00 & 2248 & 963 \\
\hline 3 & 5090 & 2783 & 3.00 & 4898 & 2650 \\
\hline 4 & 8876 & 3786 & 4.00 & 8438 & 3540 \\
\hline 5 & 13606 & 4730 & 5.00 & 12784 & 4345 \\
\hline 6 & 19225 & 5619 & 6.00 & 17857 & 5074 \\
\hline 7 & 25682 & 6457 & 7.00 & 23590 & 5733 \\
\hline 8 & 32928 & 7246 & 8.00 & 29919 & 6329 \\
\hline 8.5 & 36832 & 3904 & 9.00 & 36788 & 6869 \\
\hline 9 & 40916 & 4084 & 10.00 & 44146 & 7357 \\
\hline 9.25 & 43024 & 2108 & 11.00 & 51945 & 7799 \\
\hline 9.5 & 45175 & 2151 & 12.00 & 60143 & 8199 \\
\hline 9.75 & 47369 & 2193 & 13.00 & 68704 & 8561 \\
\hline 10 & 49604 & 2235 & 14.00 & 77592 & 8888 \\
\hline & & 49604 & 15.00 & 86776 & 9184 \\
\hline & & & 16.00 & 96228 & 9452 \\
\hline & & & 17.00 & 105922 & 9694 \\
\hline & & & 18.00 & 115836 & 9914 \\
\hline & & & 19.00 & 125948 & 10112 \\
\hline & & & 20.00 & 136240 & 10292 \\
\hline & & & 21.00 & 146695 & 10455 \\
\hline & & & 22.00 & 157296 & 10602 \\
\hline & & & 23.00 & 168031 & 10735 \\
\hline & & & 24.00 & 178886 & 10855 \\
\hline & & & 25.00 & 189850 & 10964 \\
\hline & & & 26.00 & 200913 & 11063 \\
\hline & & & 27.00 & 212065 & 11152 \\
\hline & & & 28.00 & 223297 & 11233 \\
\hline & & & 28.50 & 228941 & 5644 \\
\hline & & & 29.00 & 234603 & 5661 \\
\hline & & & 29.25 & 237440 & 2837 \\
\hline & & & 29.50 & 240281 & 2841 \\
\hline & & & 29.75 & 243126 & 2845 \\
\hline & & & 30.00 & 245974 & 2849 \\
\hline
\end{tabular}




\section{8 - As hipóteses do Programa Computacional}

O uso da formulação de elementos finitos, e a conseqüente automatização do cálculo, são essenciais para a análise de problemas oriundos da aplicação de esforços externos assimétricos à casca cilíndrica de silos e de reservatórios.

Esses esforços provocam um comportamento de difícil tratamento, onde o equacionamento via cálculo diferencial é quase não manuseável. Para grandes sistemas então, a melhor possibilidade para uma análise das equações diferenciais é via simulação numérica.

A intenção é mostrar o tipo de elemento finito utilizado, descrevendo as suas capacidades e o equacionamento que está embutido no cálculo numérico. No entanto, expor toda a teoria envolvida, inclusive a formulação do método dos elementos finitos, obviamente, não faz parte deste trabalho.

O programa computacional utilizado é a versão universitária 5.2 do ANSYS (1995). O elemento finito utilizado é chamado SHELL 63 - Elastic Shell, ou seja, um elemento de casca elástico. $\mathrm{O}$ elemento tem capacidade para operar em regime de membrana, em regime de flexão, ou em ambos. Tem seis graus de liberdade em cada nó: translações nas direções $x$, $\mathrm{y}, \mathrm{z}$ e rotações em torno dos eixos $\mathrm{x}, \mathrm{y}, \mathrm{z}$.

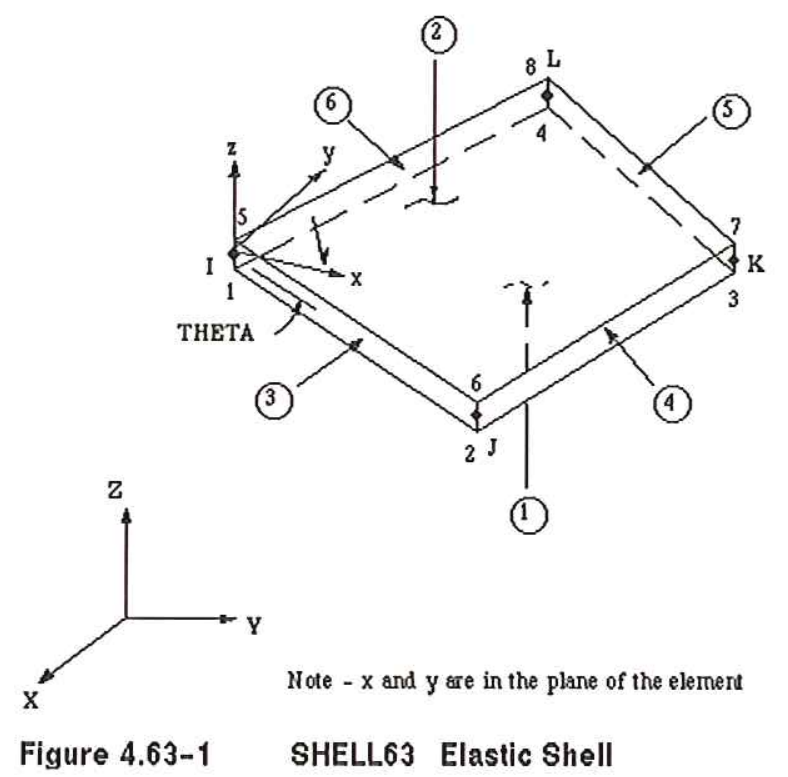

Figura 44 - O elemento finito utilizado. 
O sistema de orientação padrão do elemento tem o eixo $x$ ao longo da linha $\mathbf{i j}$, o eixo $\mathbf{y}$ perpendicular ao eixo $\mathbf{x}$ e o eixo $\mathbf{z}$ definido pela regra da mão-direita. Este é o sistema adotado neste trabalho para o elemento finito. Salienta-se, no entanto, que o sistema utilizado na análise da casca cilíndrica é o sistema cilíndrico (ver esquema da figura 32).

As solicitações externas podem ser aplicadas aos nós e às superfícies do elemento (indicados por números circulados na figura 45). A espessura do elemento é definida em cada nó e pode ser constante ou variar de nó para nó. Pode-se definir também a distância da superfície média (MID) às superfícies (1) (fundo - BOT) e (2) (topo - TOP - na direção positiva do eixo $\mathbf{z}$ ).

Os resultados estão associados aos nós em forma de deslocamentos (representam o conjunto de resultados chamado solução primária), e ao elemento (representa a chamada solução derivada). Estão ilustrados os momentos sobre a face $x\left(M_{x}\right)$, sobre a face y $\left(M_{y}\right)$ e o momento cruzado ( $\mathrm{M}_{\mathrm{Xy}}$ ). Os momentos são calculados por unidade de comprimento do sistema de coordenadas do elemento. As direções das tensões são paralelas às do sistema de coordenadas do elemento.

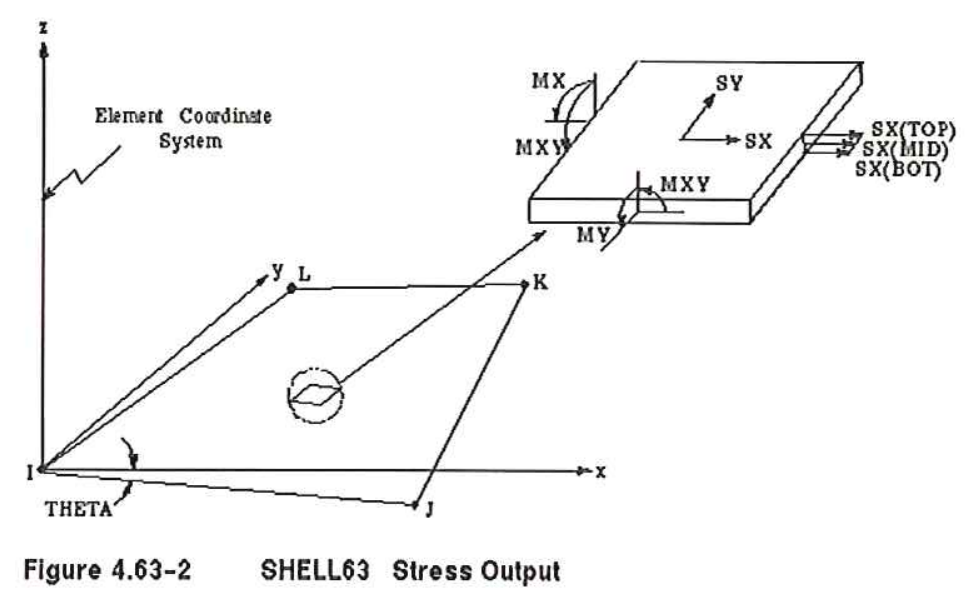

Figura 45 - Soluções no Elemento Finito.

O cálculo dos momentos ( $\mathrm{Mx}, \mathrm{My}, \mathrm{Mxy})$ e das esforços $\left(\mathrm{F}_{\mathrm{X}}, \mathrm{Fy}, \mathrm{F}_{\mathrm{Xy}}\right)$ (por unidade de comprimento do elemento) são computadas a partir das tensões definidas nas faces BOT, MID e TOP (figura 46) com:

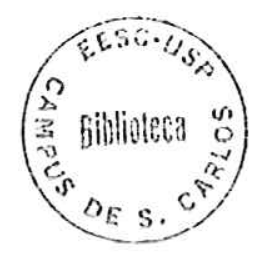




$$
\begin{aligned}
& F_{x}=\frac{e\left(\sigma_{x, \text { top }}+4 \sigma_{x, \text { mid }}+\sigma_{x, b o t}\right)}{6} \\
& F_{y}=\frac{e\left(\sigma_{y, t o p}+4 \sigma_{y, \text { mid }}+\sigma_{y, b o t}\right)}{6} \\
& F_{x y}=\frac{e\left(\sigma_{x y, t o p}+4 \sigma_{x y, \text { mid }}+\sigma_{x y, b o t}\right)}{6} \\
& M_{x}=\frac{e^{2}\left(\sigma_{x, \text { top }}-\sigma_{x, b o t}\right)}{12} \\
& M_{y}=\frac{e^{2}\left(\sigma_{y, t o p}-\sigma_{y, b o t}\right)}{12} \\
& M_{x y}=\frac{e^{2}\left(\sigma_{x y, t o p}-\sigma_{x y, b o t}\right)}{12}
\end{aligned}
$$

A solução nodal de uma análise consiste de a) a solução dos graus de liberdade, tais como deslocamentos, e b) as reações calculadas nos nós restringidos - forças, por exemplo.

As soluções tais como tensões, deformações, etc., são dadas para o centróide do elemento. Os valores são calculados como uma média dos valores nos pontos de integração, e, uma vez calculados nos pontos de integração, são extrapolados para os nós.

Em suma, o elemento SHELL 63 utiliza uma formulação em que o elemento DKT se faz presente na função de forma de deslocamento w.

\section{9 - Esquema da Análise.}

Tendo-se em vista os 2 modelos, cada modelo com 6 casos de estudo, a análise é feita comparando-se os aspectos da distribuição de tensões e dos deslocamentos de cada modelo, com o objetivo de mapear as áreas de concentração de tensões. Com base nesse estudo, é feita uma aplicação de análise de instabilidade para o exemplo de um silo de milho. A principal característica avaliada concerne à distribuição de tensões, principalmente a 
tensão meridional de compressão, ligada à variação de espessura da chapa ao longo da altura quando comparada com uma espessura constante ao longo da altura.

A análise está dividida em duas etapas:

1. Avaliação, para cada modelo, das tensões e dos deslocamentos da estrutura;

2. Comparação, entre os modelos, das regiões de concentração de tensões segundo a relação altura/diâmetro (H/D).

Foi escolhido um volume $\mathrm{V} \approx 800 \mathrm{~m}^{3}$ e as relações $\mathrm{H} / \mathrm{D}$ variando de 1 a 5 de tal forma que:

\begin{tabular}{|c|c|c|c|}
\hline \multicolumn{4}{|c|}{ TABELA 8 - Dimensões dos Modelos } \\
para o Volume de $800 \mathrm{~m}^{3}$. \\
\hline Volume & H/D & D & H \\
\hline 785 & 1 & 10 & 10 \\
\hline 848 & 5 & 6 & 30 \\
\hline
\end{tabular}

Cada modelo também teve uma distribuição de rede semelhante, com três tipos de malhas, indicadas na figura 47 :

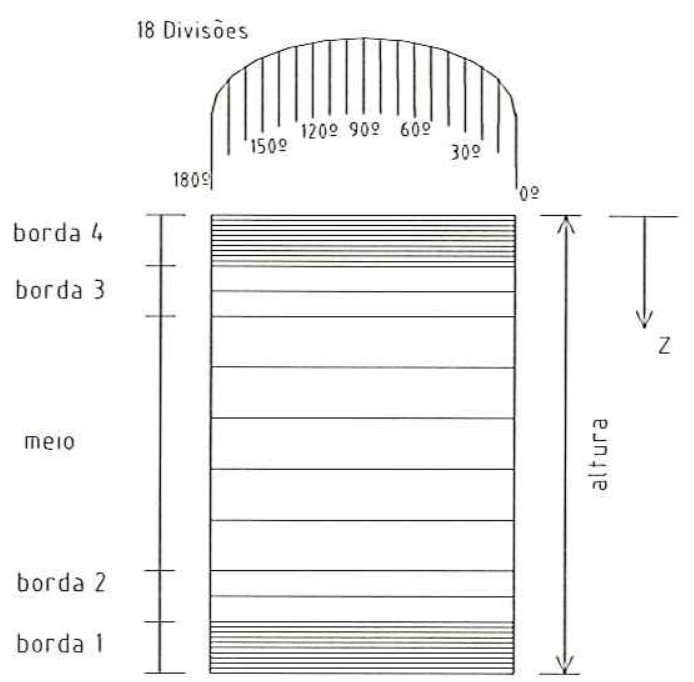

Figura 46 - Malhas e rede de elementos finitos nos modelos.

As bordas 1 e 4 têm o mesmo número de subdivisões, que é igual a 10, em uma faixa de 1 metro de altura, o que significa uma rede com malhas de $0,1 \mathrm{~m}$ de altura e largura igual ao valor do semiperímetro do modelo dividido por 18. As bordas 2 e 3 têm duas 
subdivisões em uma faixa de 1 metro de altura, que resulta em malhas de $0,5 \mathrm{~m}$ de altura $\mathrm{e}$ mesma largura que as malhas das bordas 1 e 4 . A parte do meio tem faixas de 1 metro de altura, o que dá malhas com $1 \mathrm{~m}$ de altura e mesma largura das malhas das bordas. Vale esclarecer que os elementos finitos resultam com as mesmas dimensões das malhas.

\subsection{1 - Avaliação dos esforços, tensões e deslocamentos em cada modelo.}

Cada modelo será avaliado quanto à mudança da espessura das chapas ao longo da altura para os valores dos esforços $\left(\mathrm{M}_{\mathrm{y}}\right.$ - como descrito nos elementos, segundo o esquema da figura 43), das tensões $\left(\sigma_{y}, \sigma_{z}-o\right.$ índice y indica tensão tangencial e o índice $z$ indica tensão meridional, com sinal negativo para compressão), e para os valores dos deslocamentos radiais $\left(U_{\mathrm{X}}\right)$.

O objetivo é mostrar o comportamento da casca para as diferentes espessuras adotadas. Serão mostradas as diferentes possibilidades de variação das espessuras das chapas metálicas e as faixas de extensão de cada espessura (por exemplo, chapas de 9,5 $\mathrm{mm}$ do fundo até a altura $2,0 \mathrm{~m}$, seguidas por outras de espessura $8,0 \mathrm{~mm}$, que vão de $2,0 \mathrm{~m}$ até $5 \mathrm{~m})$.

Deve ser óbvio que com os valores das tensões tangenciais não se determina o valor da espessura da chapa de aço, pois o escoamento da parede do silo não é fator determinante para o cálculo. Os valores das tensões meridionais são os valores pelos quais se deve analisar a possibilidade do fenômeno de flambagem, que está tratado no Capítulo 3 e aplicado ao exemplo do Capítulo 5.

Portanto, não é intenção modelar o comportamento ideal da casca cilíndrica, nem serem determinados valores para as tensões limites de flambagem, e sim estudar as possibilidades de comportamento, observando e descrevendo os valores das tensões e dos esforços na parede do silo ou reservatório.

Os modelos são entendidos com o auxílio de figuras tridimensionais (para o caso de comportamento assimétrico, i.e., estruturas solicitadas pelo vento), que ilustram as tensões, e com gráficos definidos para uma linha meridional (quando for o caso de comportamento axissimétrico, i.e., material ensilado ou hidrostático), que ilustram as tensões e os deslocamentos. 
Logo, para os casos 1 e 2, e os casos 3 e 4, que admitem esforços axissimétricos, as tensões tangencial e meridional, e o deslocamento radial, são apresentados em tabelas e visualizados em gráficos.

Cada figura contém 4 quadros. O primeiro é uma vista interna, da base ao topo, de metade da estrutura cilíndrica, como se o observador estivesse dentro da estrutura. O quadro 2 apresenta uma vista isométrica do lado externo da estrutura. O quadro 3 é uma vista, como se a estrutura estivesse deitada, do lado externo, com o eixo $\mathrm{z}$ na direção horizontal da página, sentido da esquerda para a direita. O quadro 4 apresenta vista externa do lado oposto ao quadro 3 , ou seja, com o eixo $\mathrm{z}$ apontado da direita para a esquerda. 
MODELO 1 - CASOS 1 e 2 - Figura 45 (Caso 1: e = constante, Caso 2 : e = variável).

Tabela 4, e Gráficos 1 e 2.
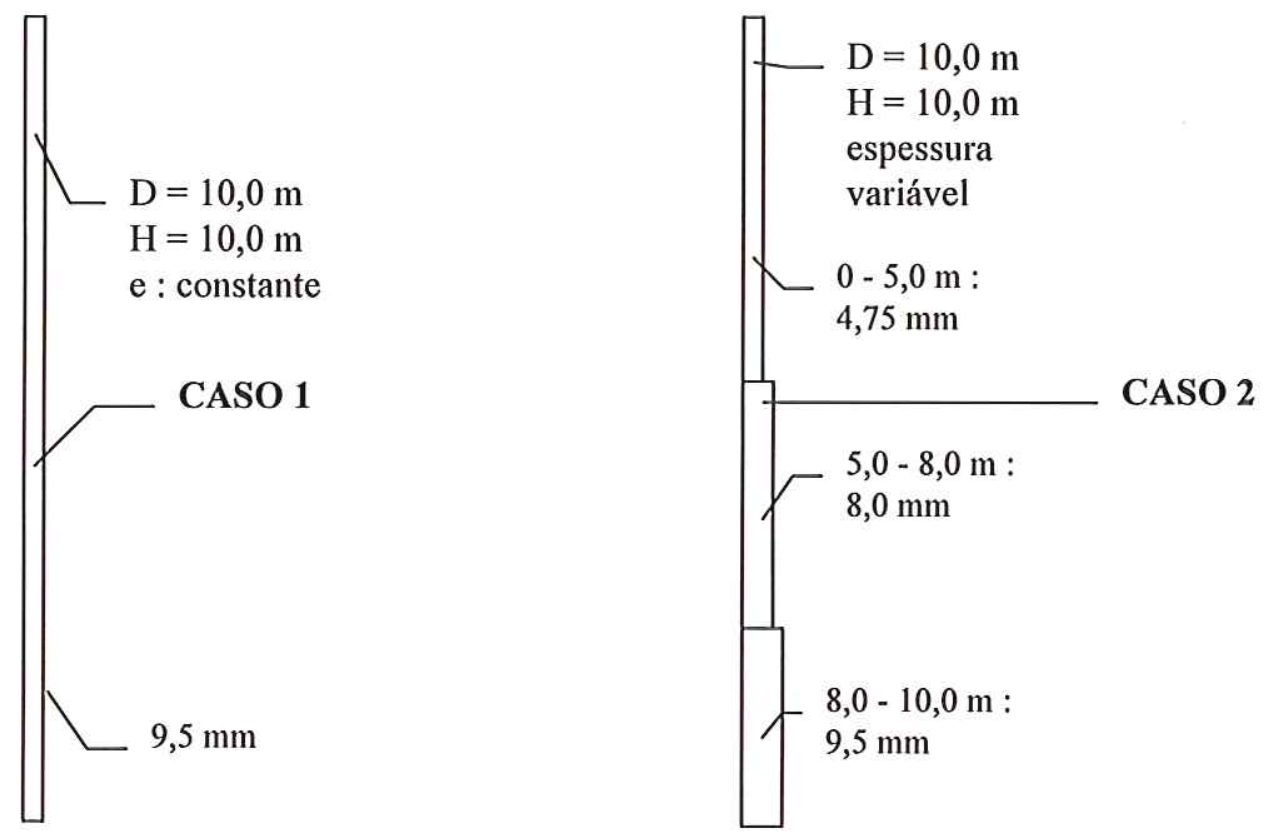

Figura 47 - Espessuras do costado para o Modelo 1 - Casos 1 e 2

No gráfico 1 , o comportamento dos deslocamentos fica evidente para a variação de espessura da parede. E, como se deve esperar, os deslocamentos são maiores no caso 2 .

Analisando-se o gráfico 2, percebe-se que a distribuição de tensões para uma espessura constante é bem graduada de acordo com a solicitação hidrostática aplicada, ou seja, as maiores tensões tangenciais aparecem mais próximas do fundo do reservatório (cota $10 \mathrm{~m}$ do gráfico 2). Quando a espessura varia, é possível obter tensões tangenciais da mesma ordem de grandeza que as do fundo em trechos mais afastados do fundo (cota 0 a $5 \mathrm{~m}$, no gráfico 2). Na cota $8.33 \mathrm{~m}$ ocorre uma mudança no comportamento das tensões tangenciais devido à mudança de espessura do costado.

Se as chapas estão bem posicionadas, ou seja, se elas se encontram a uma altura conveniente, os valores das tensões no encontro das chapas varia suavemente, como acontece na altura $5 \mathrm{~m}$ (tensão $=26 \mathrm{MPa}$ ) e na altura 4,6 (tensão $=23,8 \mathrm{MPa})$. Caso as chapas não estejam bem localizadas, ocorre uma mudança rápida, para uma distância pequena, nos valores das tensões, como acontece na altura $8,3 \mathrm{~m}$ (tensão $=43,3 \mathrm{MPa}$ ) e na altura 7,9 m (tensão = 41,4 MPa). De fato, a mudança mais brusca nos valores das tensões está localizada próximo ao fundo do reservatório, o que indica a espessura e a faixa de altura para essa espessura ( 0 a 2 m) não são adequados. 
MODELO 1

CASO 1 e CASO 2

Deslocamentos e Tensões ao Longo de uma Linha Meridional

\begin{tabular}{|c|c|c|c|c|}
\hline $\mathrm{m}$ & $\begin{array}{c}\text { UX } \\
\text { Caso } 1 \\
\text { m }\end{array}$ & $\begin{array}{c}\text { SY } \\
\text { Caso } 1 \\
\text { N/m2 } \\
\end{array}$ & $\begin{array}{c}\text { UX } \\
\text { Caso } 2 \\
\text { m }\end{array}$ & $\begin{array}{c}\text { SY } \\
\text { Caso } 2 \\
\text { N/m2 }\end{array}$ \\
\hline 0.00 & $-8.14 \mathrm{E}-08$ & -92116 & $-8.77 E-06$ & $1.62 E+06$ \\
\hline 0.21 & 2.55E-05 & $9.94 E+05$ & 3.05E-05 & $1.06 E+06$ \\
\hline 0.42 & 5.39E-05 & $2.17 E+06$ & 1.13E-04 & $1.55 E+06$ \\
\hline 0.63 & 8.07E-05 & $3.26 E+06$ & 2.02E-04 & $2.59 E+06$ \\
\hline 0.83 & 1.07E-04 & $4.33 E+06$ & $2.90 \mathrm{E}-04$ & $3.63 E+06$ \\
\hline 1.04 & 1.33E-04 & $5.40 E+06$ & $3.75 \mathrm{E}-04$ & $4.63 E+06$ \\
\hline 1.25 & $1.60 \mathrm{E}-04$ & $6.50 E+06$ & 4.60E-04 & $5.63 E+06$ \\
\hline 1.46 & 1.87E-04 & $7.60 \mathrm{E}+06$ & 5.44E-04 & $6.64 E+06$ \\
\hline 1.67 & 2.13E-04 & $8.69 E+06$ & $6.25 \mathrm{E}-04$ & $7.60 E+06$ \\
\hline 1.88 & 2.37E-04 & $9.64 E+06$ & 7.00E-04 & $8.49 E+06$ \\
\hline 2.08 & 2.61E-04 & $1.06 \mathrm{E}+07$ & $7.70 E-04$ & $9.43 E+06$ \\
\hline 2.29 & 2.89E-04 & $1.18 \mathrm{E}+07$ & 8.39E-04 & $1.05 E+07$ \\
\hline 2.50 & 3.17E-04 & $1.29 E+07$ & 9.07E-04 & $1.16 \mathrm{E}+07$ \\
\hline 2.71 & $3.45 \mathrm{E}-04$ & $1.41 \mathrm{E}+07$ & $9.75 E-04$ & $1.27 E+07$ \\
\hline 2.92 & 3.73E-04 & $1.52 E+07$ & $1.04 \mathrm{E}-03$ & $1.38 E+07$ \\
\hline 3.13 & 4.00E-04 & $1.63 E+07$ & $1.09 \mathrm{E}-03$ & $1.48 E+07$ \\
\hline 3.33 & 4.26E-04 & $1.74 E+07$ & $1.12 E-03$ & $1.59 E+07$ \\
\hline 3.54 & 4.53E-04 & $1.84 E+07$ & 1.16E-03 & $1.69 E+07$ \\
\hline 3.75 & 4.79E-04 & $1.95 \mathrm{E}+07$ & 1.19E-03 & $1.79 E+07$ \\
\hline 3.96 & $5.05 E-04$ & $2.06 E+07$ & 1.22E-03 & $1.90 E+07$ \\
\hline 4.17 & 5.32E-04 & $2.17 E+07$ & 1.24E-03 & $2.04 E+07$ \\
\hline 4.38 & $5.58 \mathrm{E}-04$ & $2.28 E+07$ & $1.25 \mathrm{E}-03$ & $2.21 E+07$ \\
\hline 4.58 & 5.85E-04 & $2.38 E+07$ & 1.27E-03 & $2.37 E+07$ \\
\hline 4.79 & $6.12 \mathrm{E}-04$ & $2.49 E+07$ & $1.28 \mathrm{E}-03$ & $2.53 E+07$ \\
\hline 5.00 & 6.38E-04 & $2.60 \mathrm{E}+07$ & $1.30 \mathrm{E}-03$ & $2.69 E+07$ \\
\hline 5.21 & $6.65 \mathrm{E}-04$ & $2.71 \mathrm{E}+07$ & $1.30 \mathrm{E}-03$ & $2.88 E+07$ \\
\hline 5.42 & 6.92E-04 & $2.82 E+07$ & 1.31E-03 & $3.07 E+07$ \\
\hline 5.63 & 7.19E-04 & $2.93 E+07$ & 1.31E-03 & $3.27 E+07$ \\
\hline 5.83 & 7.45E-04 & $3.04 E+07$ & 1.31E-03 & $3.46 \mathrm{E}+07$ \\
\hline 6.04 & 7.72E-04 & $3.14 E+07$ & 1.31E-03 & $3.64 \mathrm{E}+07$ \\
\hline 6.25 & 7.98E-04 & $3.25 E+07$ & 1.31E-03 & $3.79 E+07$ \\
\hline 6.46 & 8.25E-04 & $3.36 \mathrm{E}+07$ & 1.30E-03 & $3.93 E+07$ \\
\hline 6.67 & 8.51E-04 & $3.46 E+07$ & $1.29 \mathrm{E}-03$ & $4.07 E+07$ \\
\hline 6.88 & 8.77E-04 & $3.57 E+07$ & 1.29E-03 & $4.21 E+07$ \\
\hline 7.08 & $9.04 \mathrm{E}-04$ & $3.68 E+07$ & $1.28 \mathrm{E}-03$ & $4.33 E+07$ \\
\hline 7.29 & 9.32E-04 & $3.80 E+07$ & 1.27E-03 & $4.40 E+07$ \\
\hline 7.50 & 9.61E-04 & $3.91 \mathrm{E}+07$ & 1.27E-03 & $4.47 E+07$ \\
\hline 7.71 & $9.89 \mathrm{E}-04$ & $4.03 E+07$ & $1.26 \mathrm{E}-03$ & $4.55 \mathrm{E}+07$ \\
\hline 7.92 & $1.02 \mathrm{E}-03$ & $4.14 E+07$ & $1.26 \mathrm{E}-03$ & $4.62 E+07$ \\
\hline 8.13 & $1.04 \mathrm{E}-03$ & $4.24 E+07$ & 1.19E-03 & $4.49 E+07$ \\
\hline 8.33 & 1.07E-03 & $4.33 E+07$ & 1.12E-03 & $4.34 \mathrm{E}+07$ \\
\hline 8.54 & 1.09E-03 & $4.44 E+07$ & 1.13E-03 & $4.37 E+07$ \\
\hline 8.75 & 1.12E-03 & $4.55 E+07$ & 1.15E-03 & $4.51 E+07$ \\
\hline 8.96 & 1.14E-03 & $4.65 E+07$ & 1.16E-03 & $4.63 E+07$ \\
\hline 9.17 & 1.18E-03 & $4.77 E+07$ & 1.19E-03 & $4.79 E+07$ \\
\hline 9.38 & $1.24 \mathrm{E}-03$ & $5.08 E+07$ & $1.25 \mathrm{E}-03$ & $5.01 E+07$ \\
\hline 9.58 & 1.25E-03 & $5.42 E+07$ & $1.25 \mathrm{E}-03$ & $4.74 \mathrm{E}+07$ \\
\hline 9.79 & $8.17 \mathrm{E}-04$ & $3.85 \mathrm{E}+07$ & 8.16E-04 & $2.78 \mathrm{E}+07$ \\
\hline 10.00 & $-2.03 E-18$ & $-2.49 E+07$ & $-2.03 E-18$ & $2.46 E+07$ \\
\hline
\end{tabular}

Tabela 9 - Deslocamentos e Tensões - Modelo 1 - Casos 1 e 2. 
GRÁFICO 1 - Deslocamentos Radiais - MODELO 1 - Casos 1 e 2

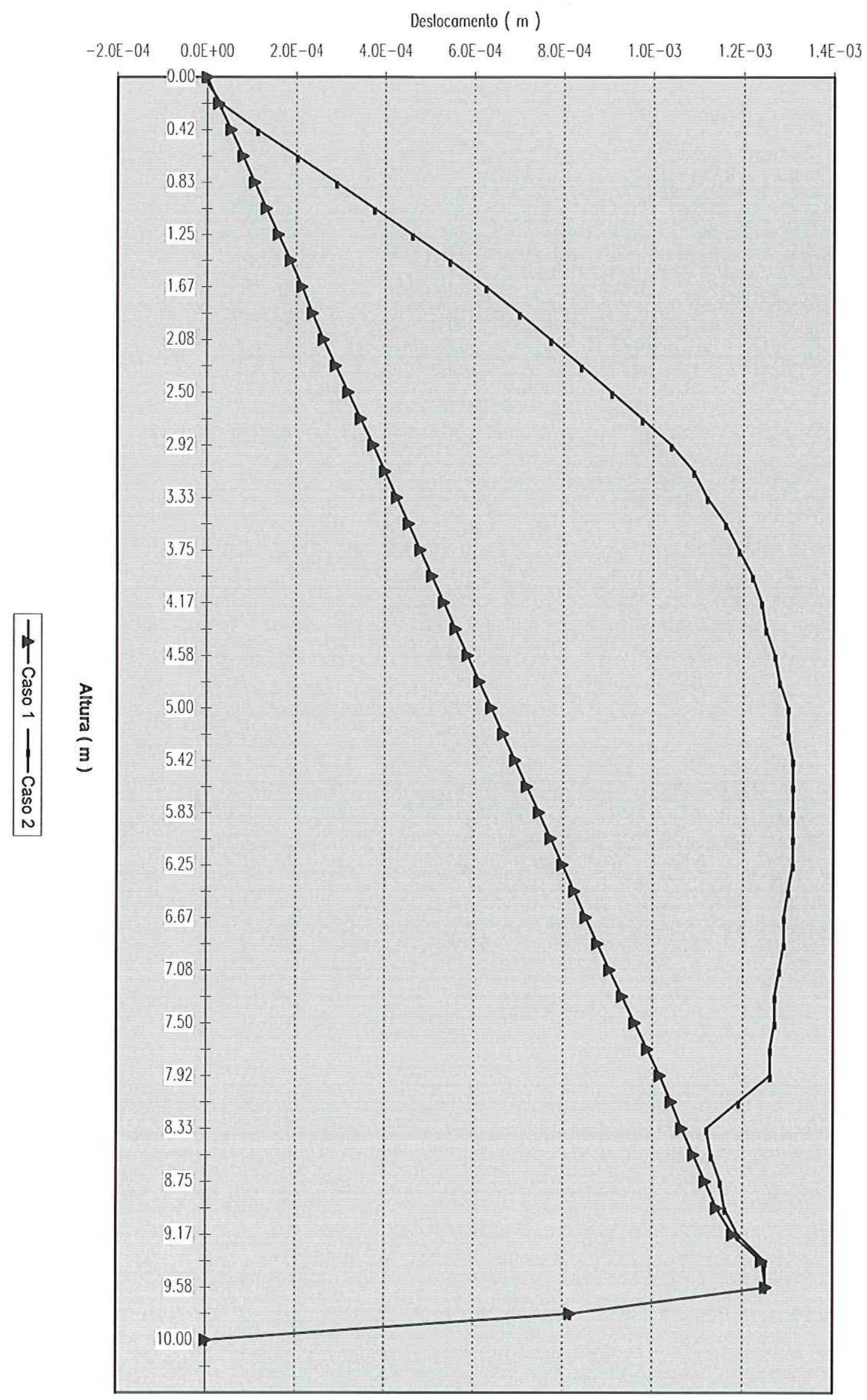


GRÁFICO 2 - Tensões Tangenciais - MODELO 1 - Casos 1 e 2

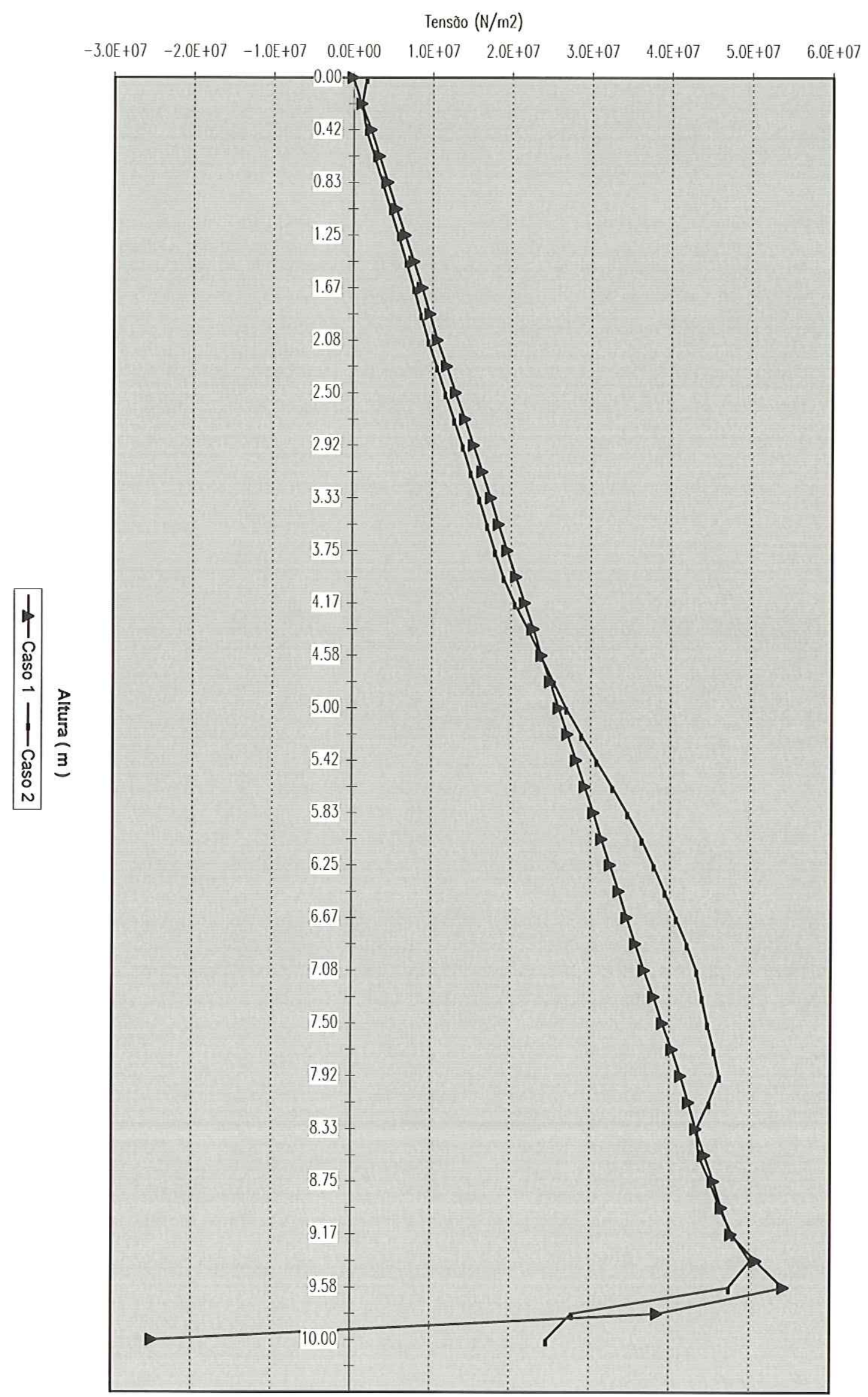


MODELO 1 - CASOS 3 e 4, Figura 45 (Caso 3: e = constante, Caso 4 : e = variável).

Tabela 5, e Gráficos 3 e 4.
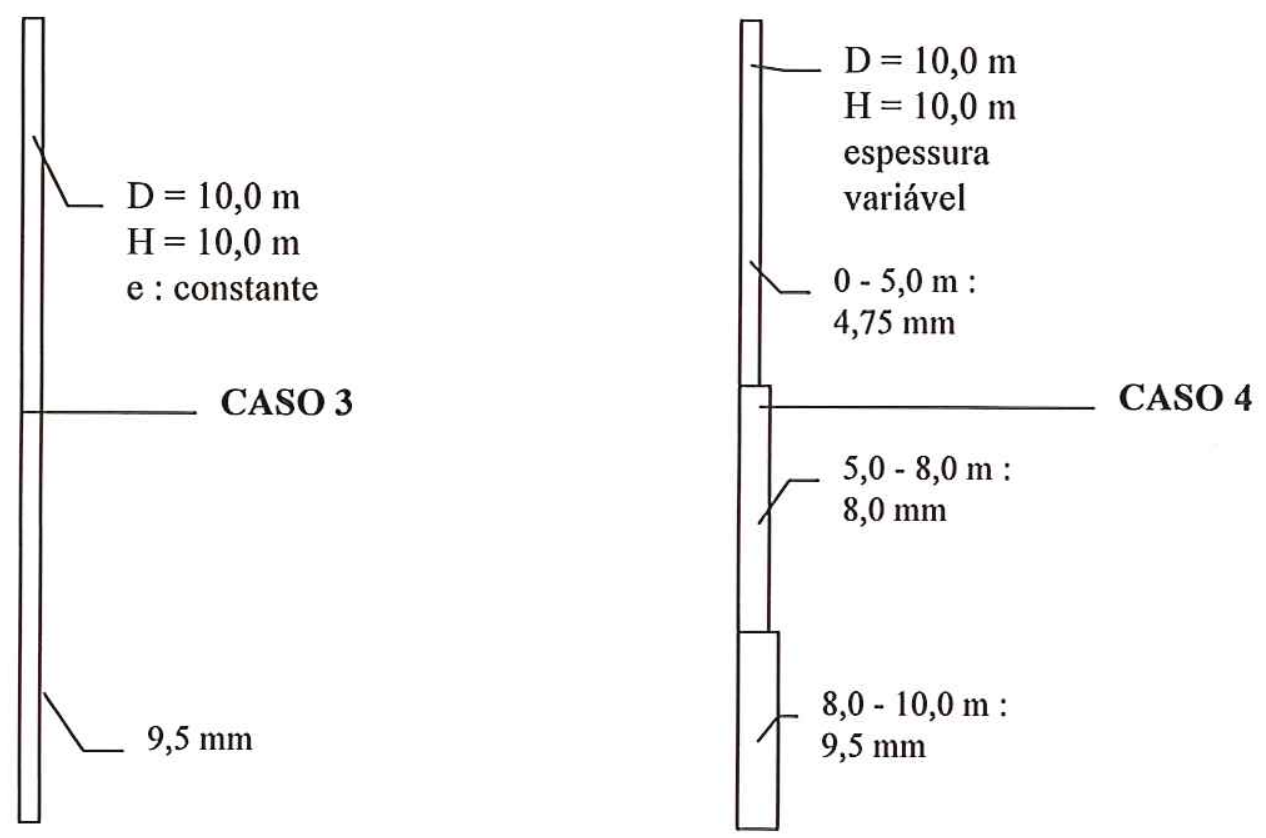

Figura 48 - Espessuras do costado para o Modelo 1 - Casos 3 e 4

Os deslocamentos radiais, no gráfico 3, para o caso de material ensilado, apresenta maior variação de deslocamentos que o caso hidrostático; a curva correspondente ao caso 3 é praticamente linear no trecho central, com perturbações nas regiões próximas às bordas; a curva correspondente ao caso 4 tem um comportamento tortuoso, reflexo da atuação do atrito do material ensilado com a parede do silo.

O gráfico 4 reflete as condições dos deslocamentos, tanto para o caso 3 quanto para o caso 4, apresentado configurações semelhantes às dos deslocamentos radiais.

O comportamento das tensões meridionais é suave para o caso de espessura constante (Caso 3), com um aumento de valores exponencial. Para a curva correspondente ao caso 4, com parede de espessura variável, ocorrem mudanças de inclinação da curva par os pontos de variação de espessura da chapa. Essas mudanças de espessura, para este caso, não chegam a mudar de modo considerável o comportamento da curva do caso 4 , pois a curva não apresenta inclinações fortes.

- NOTA: A inclinação de uma curva deve ser entendida como a inclinação da reta tangente ao ponto da curva, como definido em cálculo diferencial. 
MODELO 1

CASO 3 e CASO 4

Deslocamentos e Tensőes ao Longo de uma Linha Meridional.

\begin{tabular}{|c|c|c|c|c|c|c|}
\hline $\mathrm{m}$ & $\begin{array}{c}\text { UX } \\
\text { Caso } 3 \\
\text { m }\end{array}$ & $\begin{array}{c}\text { UX } \\
\text { Caso } 4 \\
\text { m }\end{array}$ & $\begin{array}{c}\text { SY } \\
\text { Caso } 3 \\
\text { N/m2 }\end{array}$ & $\begin{array}{c}\text { SY } \\
\text { Caso } 4 \\
\text { N/m2 }\end{array}$ & $\begin{array}{c}\text { SZ } \\
\text { Caso } 3 \\
\mathrm{~N} / \mathrm{m} 2\end{array}$ & $\begin{array}{c}\text { SZ } \\
\text { Caso } 4 \\
\text { N/m2 }\end{array}$ \\
\hline 0.00 & 2.33E-06 & 1.82E-06 & $-1.52 E+05$ & $-2.35 E+05$ & -1335.5 & $-2.33 E+05$ \\
\hline 0.21 & 5.32E-05 & $1.21 \mathrm{E}-04$ & $1.97 \mathrm{E}+06$ & $4.50 E+06$ & -2971 & $-3.09 E+05$ \\
\hline 0.42 & $6.08 \mathrm{E}-05$ & $1.24 \mathrm{E}-04$ & $2.34 \mathrm{E}+06$ & $4.58 \mathrm{E}+06$ & -3857.5 & $-3.07 E+05$ \\
\hline 0.63 & 5.60E-05 & 1.19E-04 & $2.20 \mathrm{E}+06$ & $4.30 E+06$ & -4689.7 & $-3.02 E+05$ \\
\hline 0.83 & $6.10 \mathrm{E}-05$ & $1.28 E-04$ & $2.47 \mathrm{E}+06$ & $4.62 E+06$ & -5936.8 & $-2.93 E+05$ \\
\hline 1.04 & 8.37E-05 & 1.89E-04 & $3.44 \mathrm{E}+06$ & $7.02 E+06$ & -43795 & $-3.54 E+05$ \\
\hline 1.25 & 1.05E-04 & $2.40 \mathrm{E}-04$ & $4.36 E+06$ & $9.02 E+06$ & -71406 & $-3.97 E+05$ \\
\hline 1.46 & 1.02E-04 & 2.32E-04 & $4.30 \mathrm{E}+06$ & $8.65 E+06$ & $-1.07 E+05$ & $-4.52 E+05$ \\
\hline 1.67 & 9.99E-05 & 2.25E-04 & $4.29 E+06$ & $8.31 \mathrm{E}+06$ & $-1.44 E+05$ & $-5.10 E+05$ \\
\hline 1.88 & 1.12E-04 & 2.60E-04 & 4.87E+06 & $9.69 E+06$ & $-1.93 E+05$ & $-5.88 E+05$ \\
\hline 2.08 & $1.28 \mathrm{E}-04$ & 3.03E-04 & $5.59 \mathrm{E}+06$ & $1.14 E+07$ & $-2.46 E+05$ & $-6.77 E+05$ \\
\hline 2.29 & 1.36E-04 & $3.17 \mathrm{E}-04$ & $5.95 \mathrm{E}+06$ & $1.20 \mathrm{E}+07$ & $-2.92 E+05$ & $-7.64 E+05$ \\
\hline 2.50 & 1.43E-04 & $3.31 \mathrm{E}-04$ & $6.32 \mathrm{E}+06$ & $1.26 \mathrm{E}+07$ & $-3.39 E+05$ & $-8.50 E+05$ \\
\hline 2.71 & 1.50E-04 & $3.45 \mathrm{E}-04$ & $6.69 \mathrm{E}+06$ & $1.31 \mathrm{E}+07$ & $-3.85 E+05$ & $-9.37 E+05$ \\
\hline 2.92 & $1.58 \mathrm{E}-04$ & 3.59E-04 & $7.05 \mathrm{E}+06$ & $1.37 E+07$ & $-4.32 E+05$ & $-1.02 E+06$ \\
\hline 3.13 & 1.66E-04 & $3.81 \mathrm{E}-04$ & $7.43 E+06$ & $1.47 \mathrm{E}+07$ & $-4.96 E+05$ & $-1.15 E+06$ \\
\hline 3.33 & 1.75E-04 & 4.10E-04 & $7.82 E+06$ & $1.58 \mathrm{E}+07$ & $-5.71 E+05$ & $-1.30 E+06$ \\
\hline 3.54 & 1.83E-04 & 4.39E-04 & $8.21 \mathrm{E}+06$ & $1.70 E+07$ & $-6.46 E+05$ & $-1.45 E+06$ \\
\hline 3.75 & 1.92E-04 & $4.68 \mathrm{E}-04$ & $8.60 \mathrm{E}+06$ & $1.82 E+07$ & $-7.21 E+05$ & $-1.60 E+06$ \\
\hline 3.96 & 2.01E-04 & 4.96E-04 & $8.99 E+06$ & $1.94 E+07$ & $-7.96 E+05$ & $-1.75 E+06$ \\
\hline 4.17 & 2.10E-04 & 4.86E-04 & $9.35 E+06$ & $1.91 E+07$ & $-8.85 E+05$ & $-1.82 E+06$ \\
\hline 4.38 & 2.19E-04 & 4.67E-04 & $9.71 \mathrm{E}+06$ & $1.83 E+07$ & $-9.78 E+05$ & $-1.88 E+06$ \\
\hline 4.58 & $2.28 \mathrm{E}-04$ & 4.47E-04 & $1.01 \mathrm{E}+07$ & $1.76 \mathrm{E}+07$ & $-1.07 E+06$ & $-1.93 E+06$ \\
\hline 4.79 & 2.37E-04 & 4.27E-04 & $1.04 \mathrm{E}+07$ & $1.68 E+07$ & $-1.16 E+06$ & $-1.99 E+06$ \\
\hline 5.00 & $2.47 \mathrm{E}-04$ & $4.07 \mathrm{E}-04$ & $1.08 \mathrm{E}+07$ & $1.61 \mathrm{E}+07$ & $-1.26 E+06$ & $-2.04 \mathrm{E}+06$ \\
\hline 5.21 & $2.56 E-04$ & $3.95 E-04$ & $1.11 \mathrm{E}+07$ & $1.56 \mathrm{E}+07$ & $-1.36 E+06$ & $-2.08 E+06$ \\
\hline 5.42 & 2.66E-04 & $3.82 E-04$ & 1.15E+07 & $1.51 \mathrm{E}+07$ & $-1.47 E+06$ & $-2.13 E+06$ \\
\hline 5.63 & 2.76E-04 & $3.70 \mathrm{E}-04$ & $1.18 \mathrm{E}+07$ & $1.46 \mathrm{E}+07$ & $-1.58 E+06$ & $-2.17 \mathrm{E}+06$ \\
\hline 5.83 & 2.86E-04 & 3.57E-04 & $1.21 \mathrm{E}+07$ & $1.42 \mathrm{E}+07$ & $-1.69 E+06$ & $-2.21 E+06$ \\
\hline 6.04 & 2.96E-04 & 3.51E-04 & $1.25 E+07$ & $1.39 E+07$ & $-1.81 E+06$ & $-2.27 E+06$ \\
\hline 6.25 & 3.06E-04 & 3.69E-04 & $1.28 \mathrm{E}+07$ & $1.46 \mathrm{E}+07$ & $-1.93 E+06$ & $-2.42 E+06$ \\
\hline 6.46 & 3.17E-04 & 3.87E-04 & $1.31 \mathrm{E}+07$ & $1.53 E+07$ & $-2.06 E+06$ & $-2.58 E+06$ \\
\hline 6.67 & $3.27 \mathrm{E}-04$ & 4.05E-04 & $1.35 E+07$ & $1.60 \mathrm{E}+07$ & $-2.19 E+06$ & $-2.73 E+06$ \\
\hline 6.88 & $3.38 \mathrm{E}-04$ & 4.23E-04 & $1.38 \mathrm{E}+07$ & $1.67 \mathrm{E}+07$ & $-2.32 E+06$ & $-2.88 E+06$ \\
\hline 7.08 & $3.48 \mathrm{E}-04$ & 4.35E-04 & $1.41 \mathrm{E}+07$ & $1.71 \mathrm{E}+07$ & $-2.45 E+06$ & $-3.01 E+06$ \\
\hline 7.29 & 3.57E-04 & 4.37E-04 & $1.44 \mathrm{E}+07$ & $1.72 \mathrm{E}+07$ & $-2.60 E+06$ & $-3.12 E+06$ \\
\hline 7.50 & $3.67 \mathrm{E}-04$ & $4.39 \mathrm{E}-04$ & $1.47 \mathrm{E}+07$ & $1.72 \mathrm{E}+07$ & $-2.74 \mathrm{E}+06$ & $-3.22 E+06$ \\
\hline 7.71 & 3.76E-04 & $4.41 \mathrm{E}-04$ & $1.49 \mathrm{E}+07$ & $1.72 \mathrm{E}+07$ & $-2.89 E+06$ & $-3.32 E+06$ \\
\hline 7.92 & 3.86E-04 & 4.43E-04 & $1.52 E+07$ & $1.73 E+07$ & $-3.04 E+06$ & $-3.43 E+06$ \\
\hline 8.13 & 4.02E-04 & 4.31E-04 & $1.57 \mathrm{E}+07$ & 1.67E+07 & $-3.28 E+06$ & $-3.52 E+06$ \\
\hline 8.33 & 4.16E-04 & 4.17E-04 & $1.62 \mathrm{E}+07$ & $1.61 \mathrm{E}+07$ & $-3.53 E+06$ & $-3.63 E+06$ \\
\hline 8.54 & 4.18E-04 & $4.18 E-04$ & $1.62 E+07$ & $1.60 E+07$ & $-3.70 E+06$ & $-3.80 E+06$ \\
\hline 8.75 & 4.19E-04 & 4.21E-04 & $1.61 \mathrm{E}+07$ & $1.61 \mathrm{E}+07$ & $-3.86 E+06$ & $-3.96 E+06$ \\
\hline 8.96 & 4.36E-04 & 4.37E-04 & 1.67E+07 & 1.67E+07 & $-4.04 E+06$ & $-4.14 E+06$ \\
\hline 9.17 & $4.58 \mathrm{E}-04$ & 4.59E-04 & $1.74 \mathrm{E}+07$ & $1.74 \mathrm{E}+07$ & $-4.28 E+06$ & $-4.38 E+06$ \\
\hline 9.38 & 4.79E-04 & $4.80 \mathrm{E}-04$ & $1.82 E+07$ & $1.82 \mathrm{E}+07$ & $-4.47 E+06$ & $-4.57 E+06$ \\
\hline 9.58 & 4.74E-04 & 4.74E-04 & $1.79 E+07$ & $1.79 \mathrm{E}+07$ & $-4.70 E+06$ & $-4.80 E+06$ \\
\hline 9.79 & 3.05E-04 & 3.06E-04 & $1.09 \mathrm{E}+07$ & $1.09 E+07$ & $-4.94 E+06$ & $-5.03 E+06$ \\
\hline 10.00 & $-7.54 \mathrm{E}-19$ & $-7.55 E-19$ & $-1.56 E+06$ & $-1.58 E+06$ & $-5.18 E+06$ & $-5.27 E+06$ \\
\hline
\end{tabular}

Tabela 10 - Deslocamentos e Tensões - Modelo1 - Casos 3 e 4 
GRÁFICO 3 - Deslocamentos Radiais - MODELO 1 - Casos 3 e 4

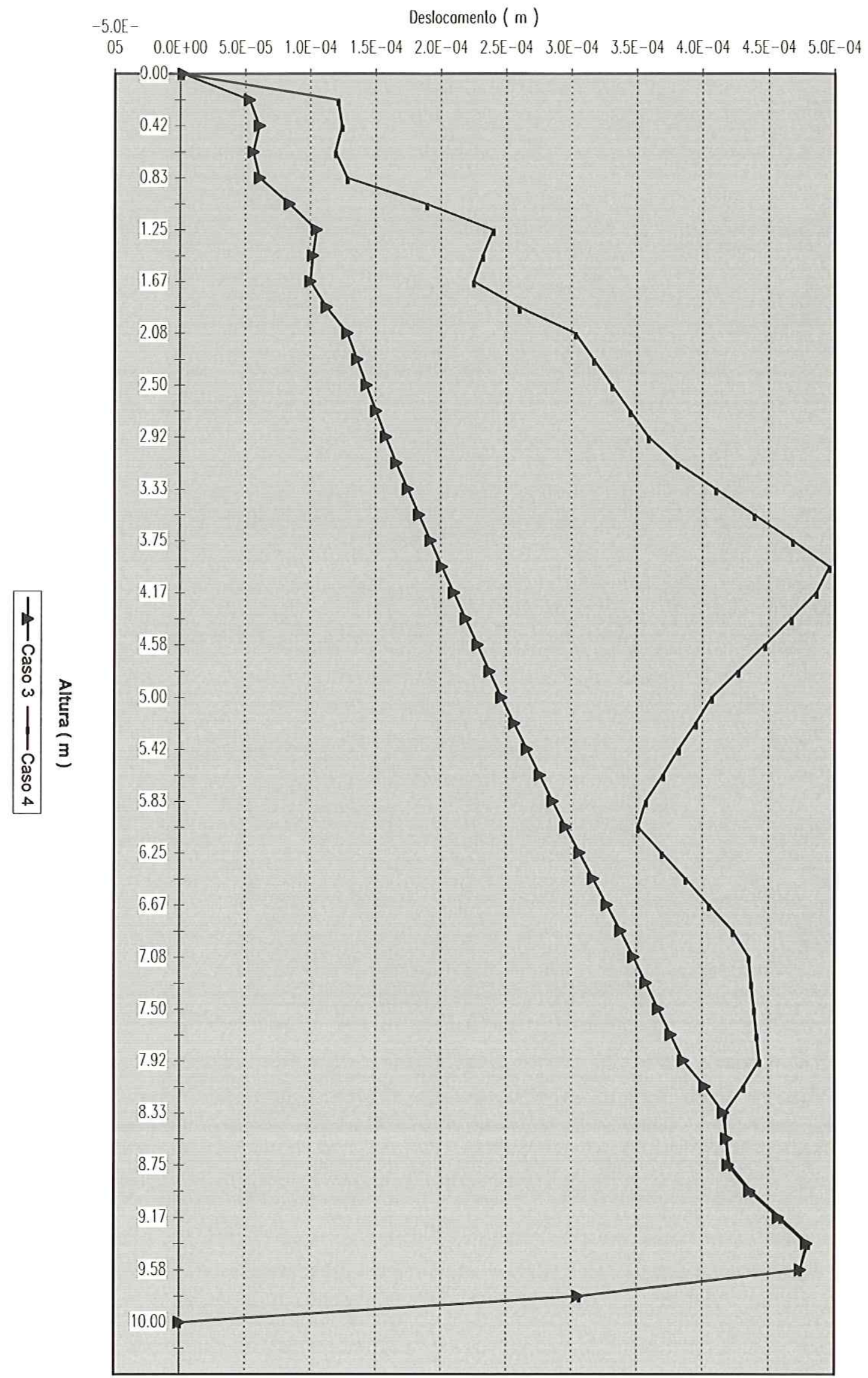


GRÁFICO 4 - Tensões Tangenciais - MODELO 1 - Casos 3 e 4

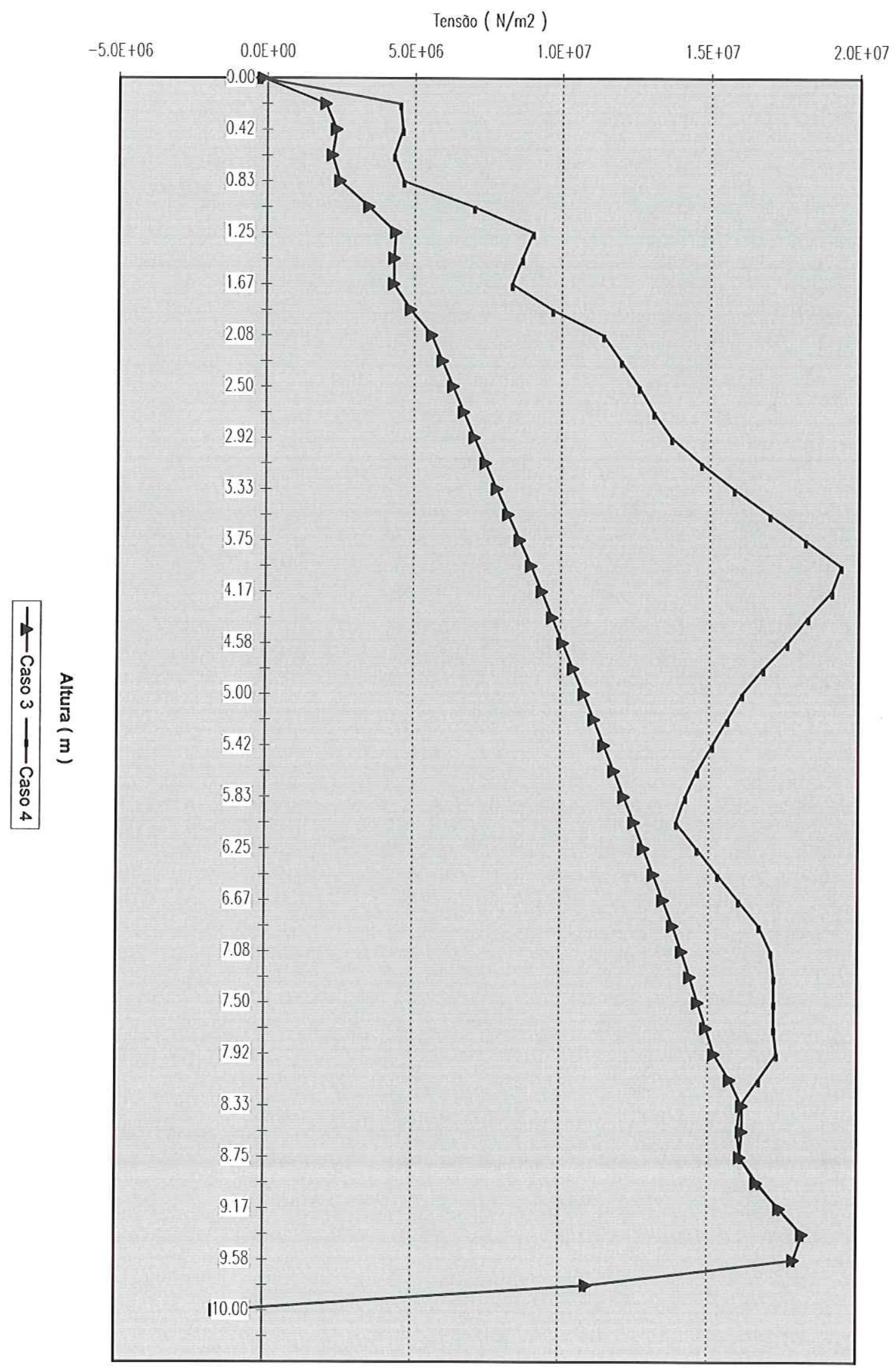


GRÁFICO 5 - Tensões Meridionais - MODELO 1 - Casos 3 e 4

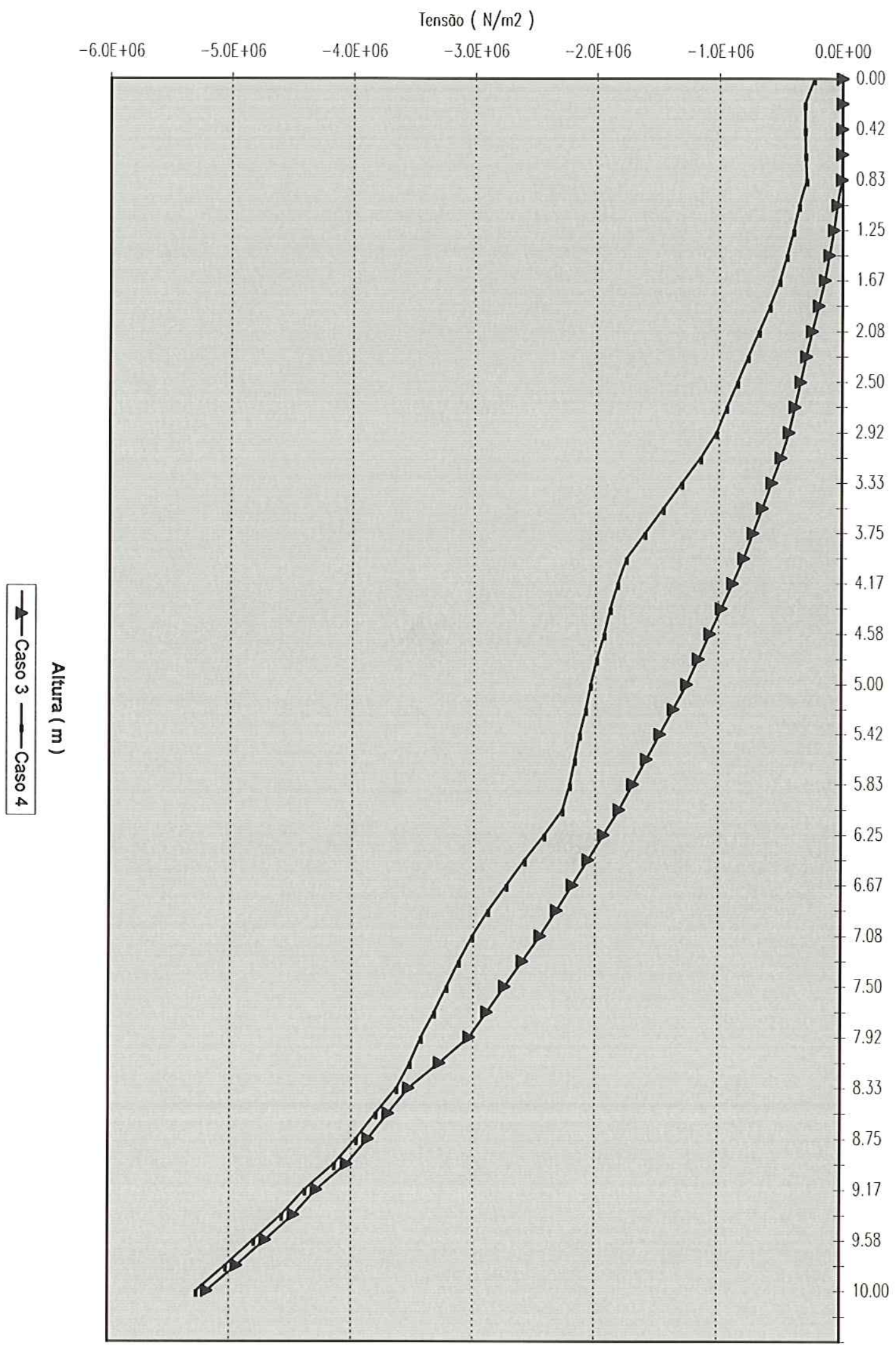


MODELO 1 : CASOS 5 e 6 - Figuras 49-50 (Caso 5) - Tensões em Pa.
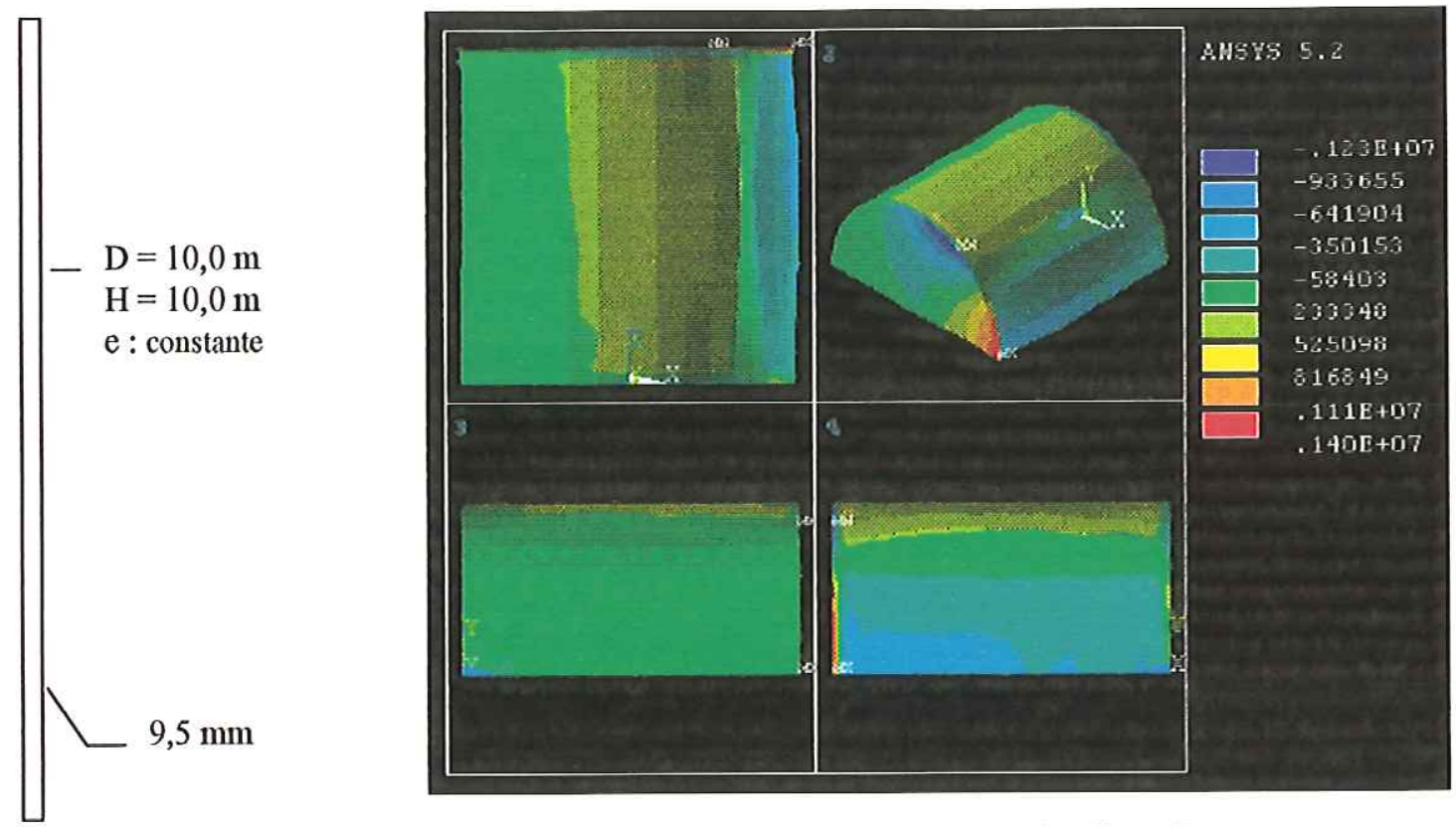

Figura 49 - Tensões tangenciais $\sigma_{y}-$ Modelo 1 - Caso 5
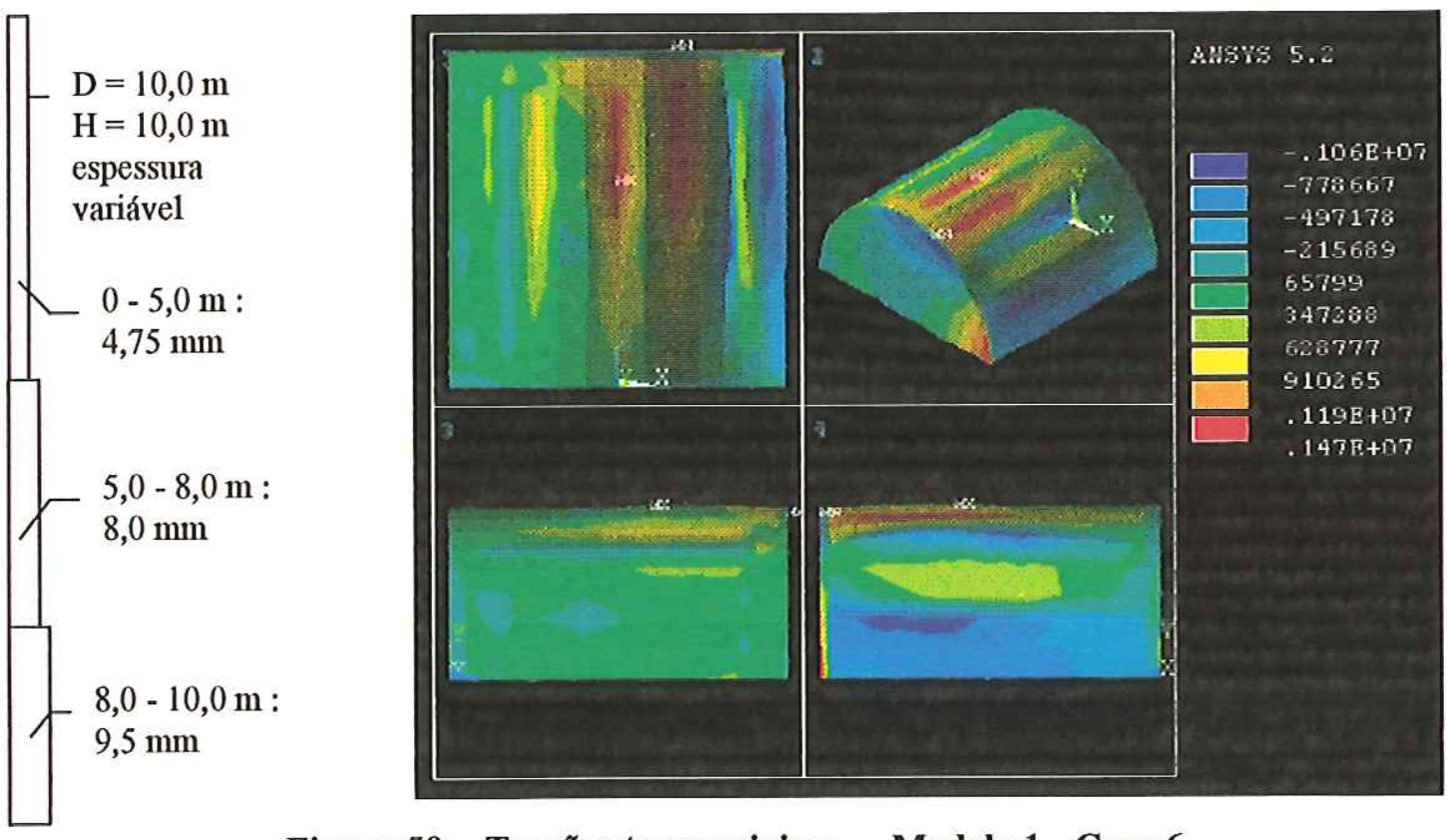

Figura 50 - Tensões tangenciais $\sigma_{y}$ - Modelo 1 - Caso 6.

Nas figuras 49 e 50 estão mostradas as tensões tangenciais para a solicitação do vento. As maiores tensões estão localizadas próximas ao topo, com duas regiões distintas em vemelho e em azul. Isto corresponde à atuação, na região em azul, de pressão positiva do vento (ou sobrepressões ); a região em vermelho corresponde ao efeito de suç̧ão do vento. Dependendo da intensidade de atuação das forças do vento, pode ocorrer o fenômeno de ovalização da seção transversal da casca. Este ponto da análise fundamenta-se em observações feitas em Gaylord \& Gaylord (1985). 
MODELO 1 : CASOS 5 e 6 - Figuras 51-52 (Caso 6)
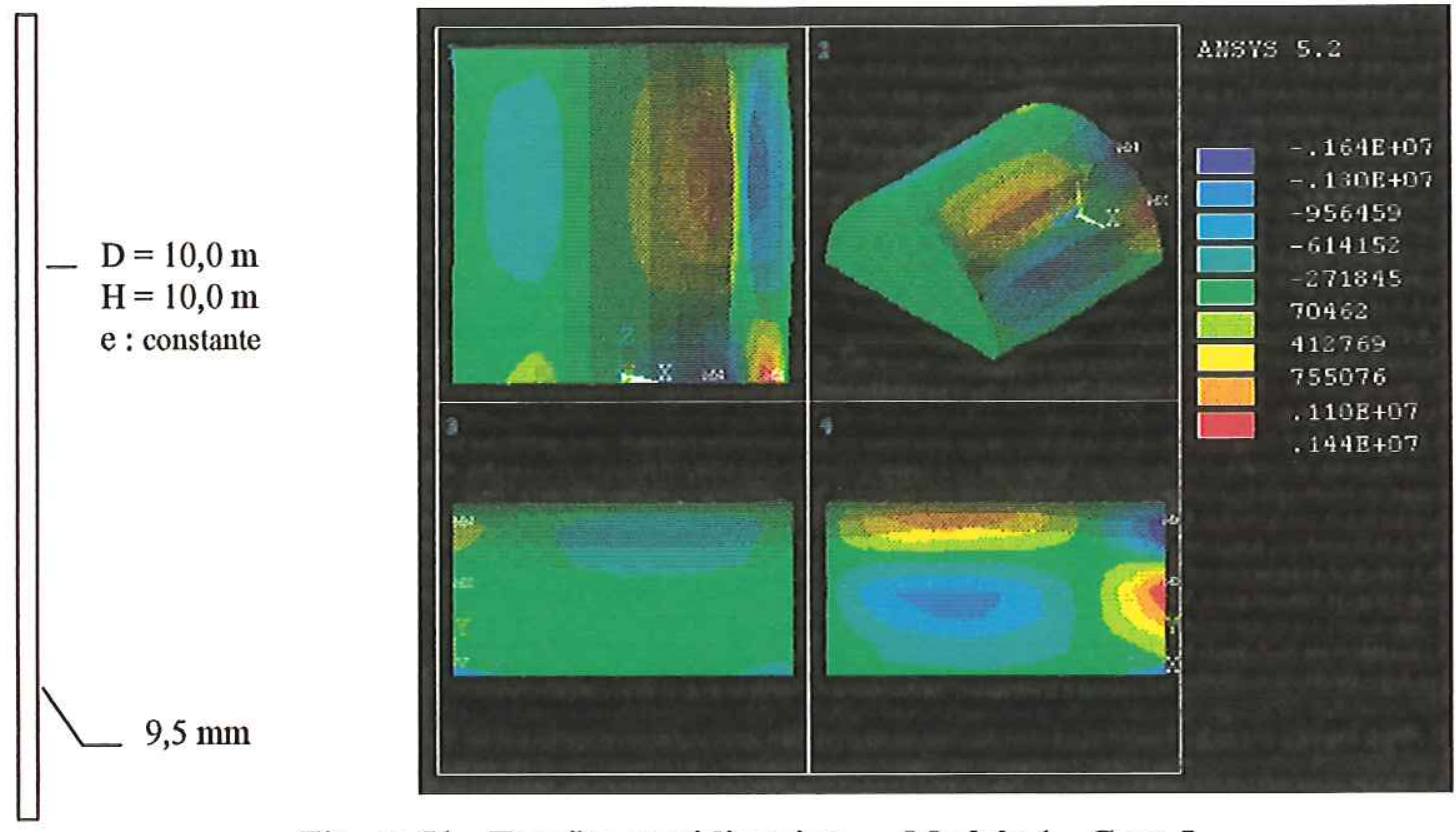

Figura 51 - Tensões meridionais $\sigma_{Z}-$ Modelo 1 - Caso 5
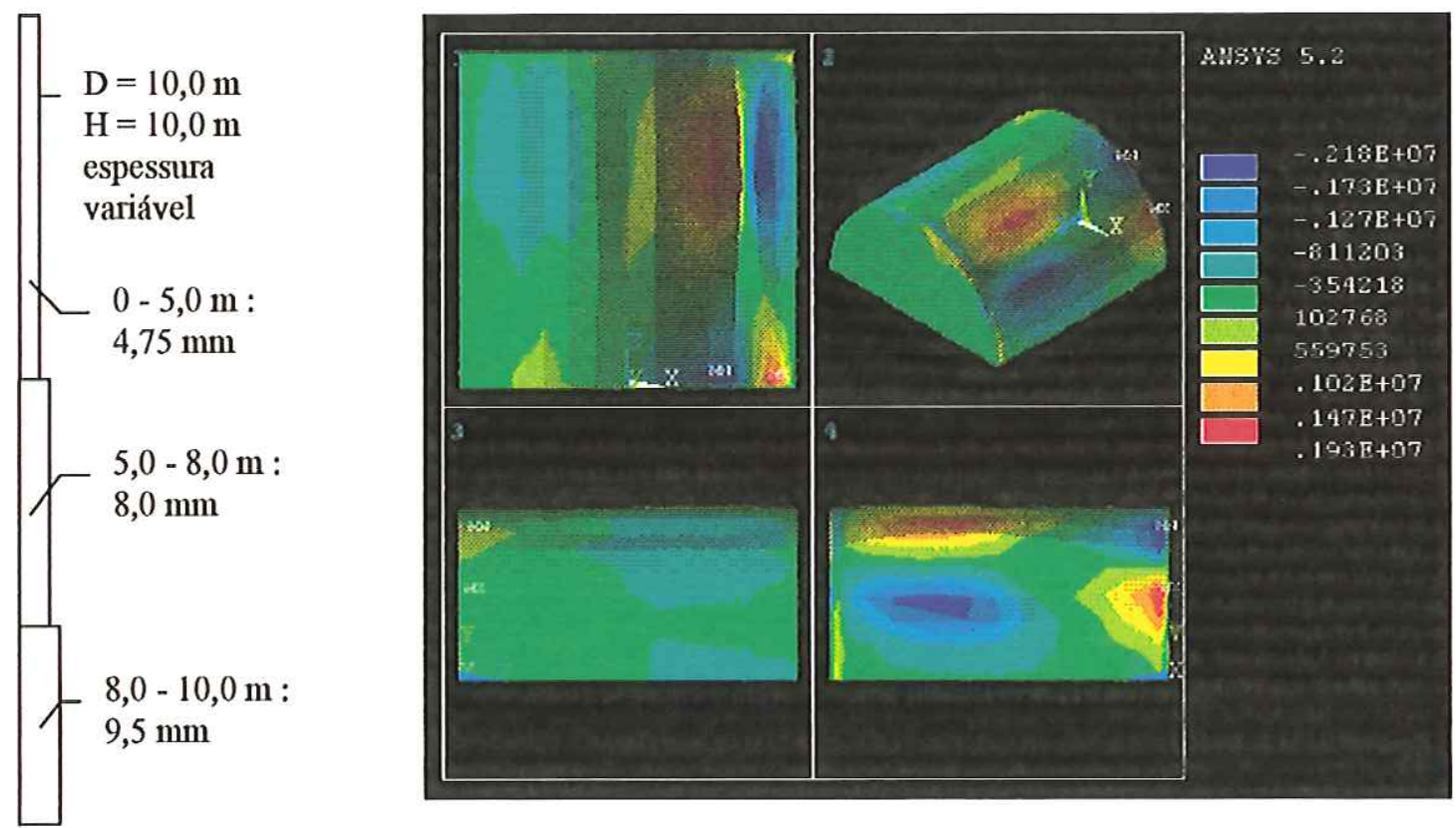

Figura 52 - Tensões meridionais $\sigma_{\mathrm{Z}}-$ Modelo 1 - Caso 6.

As figuras 51 e 52 apresentam regiões em azul, ou seja, maiores tensões de compressão, em duas regiões distintas: uma próxima ao topo, e outra junto à base. A região próxima ao topo indica cuidado em relação à atuação do vento a barlavento, na região de sobrepressão do vento. A região em azul próxima à base, alerta para um possível cuidado em 
relação à compressão, no entanto é de menor importância em relação à região próxima à tampa porque geralmente a base apresenta espessura maior.

A região em vermelho próxima à base, na região a barlavento, apresenta os maiores valores de tração meridional. É nesta região que se devem anotar os valores para o cálculo da ancoragem da estrutura ao vento. A outra região em vermelho, mais próxima da tampa, se vista em par com a região próxima à base, na realidade mostra como a estrutura está sendo solicitada: ocorre um efeito de arrancamento (na estrutura) em relação ao plano da base.

A região a sotavento não apresenta maiores tensões que a região a barlavento. Aliás, isto não significa muito, pois o vento pode atuar vindo de qualquer direção, o que indica ser necessário considerar a estrutura para a situação mais desfavorável em todo o perímetro.

Comparando-se as regiões de tensões para o caso da espessura constante e o caso da espessura variável, nota-se um aumento dos valores para a região de menor espessura (figura 50, na faixa de 6 a $10 \mathrm{~m}$ de altura). A localização das regiões de tensão, entretanto, não varia significativamente. 
MODELO 5 - CASOS 1 e 2 - Figura 53 (Caso $1: \mathrm{e}=$ constante, Caso 2 : e = variável).
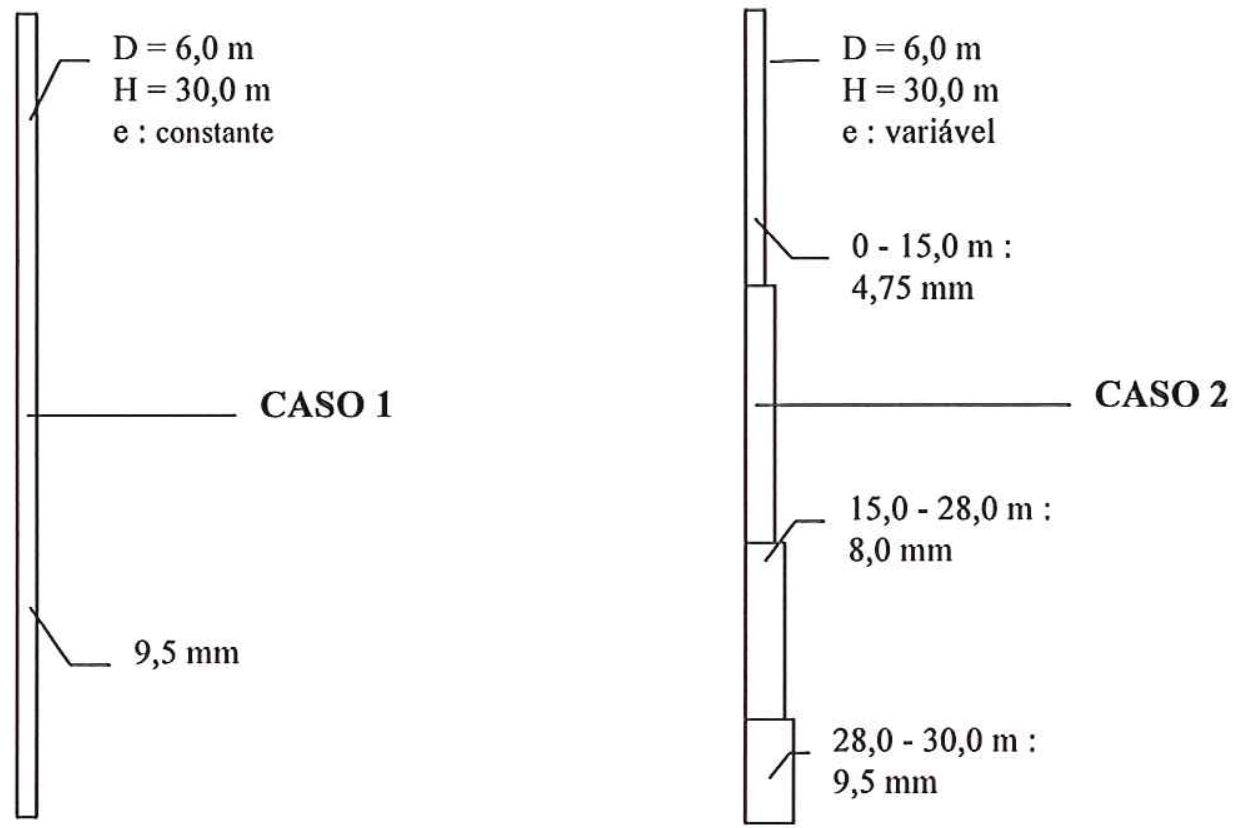

Figura 53 - Espessuras do Costado para o Modelo 5 - Casos 1 e 2.

Os deslocamentos radiais para o Caso $\mathbf{1}$, no gráfico 6 , seguem um padrão linear devido à solicitação hidrostática, como nos outros 4 modelos. Para o Caso 2, no gráfico 7, também nada de novo ocorre: há picos de deslocamentos onde existem mudanças de espessuras. 
MODELO 5

CASO 1 e CASO 2

Deslocamentos e Tensőes ao Longo de uma Linha Meridional

\begin{tabular}{|c|c|c|c|c|}
\hline$S$ & $\begin{array}{c}\text { UX } \\
\text { Caso } 1 \\
\text { m }\end{array}$ & $\begin{array}{c}\text { UX } \\
\text { Caso } 2 \\
\text { m }\end{array}$ & $\begin{array}{c}\text { SY } \\
\text { Caso } 1 \\
\mathrm{~N} / \mathrm{m} 2\end{array}$ & $\begin{array}{c}\text { Sy } \\
\text { Caso } 2 \\
\text { N/m2 }\end{array}$ \\
\hline 0.00 & $4.55 \mathrm{E}-06$ & $1.88 \mathrm{E}-05$ & $3.08 \mathrm{E}+05$ & 2131.7 \\
\hline 0.62 & 3.33E-05 & $7.58 \mathrm{E}-05$ & $2.26 \mathrm{E}+06$ & $3.90 E+06$ \\
\hline 1.25 & $6.20 \mathrm{E}-05$ & 1.33E-04 & $4.20 \mathrm{E}+06$ & $7.81 \mathrm{E}+06$ \\
\hline 1.87 & 8.88E-05 & $1.88 \mathrm{E}-04$ & $6.02 E+06$ & $1.16 \mathrm{E}+07$ \\
\hline 2.49 & 1.19E-04 & $2.45 \mathrm{E}-04$ & $8.06 \mathrm{E}+06$ & $1.55 \mathrm{E}+07$ \\
\hline 3.11 & $1.48 \mathrm{E}-04$ & 3.05E-04 & $1.01 \mathrm{E}+07$ & $1.96 E+07$ \\
\hline 3.74 & 1.77E-04 & 3.61E-04 & $1.20 \mathrm{E}+07$ & $2.34 \mathrm{E}+07$ \\
\hline 4.36 & 2.05E-04 & $4.18 \mathrm{E}-04$ & $1.39 \mathrm{E}+07$ & $2.73 E+07$ \\
\hline 4.98 & $2.34 \mathrm{E}-04$ & 4.75E-04 & $1.59 \mathrm{E}+07$ & $3.12 E+07$ \\
\hline 5.61 & $2.63 \mathrm{E}-04$ & 5.31E-04 & $1.78 \mathrm{E}+07$ & $3.51 \mathrm{E}+07$ \\
\hline 6.23 & 2.91E-04 & $5.89 \mathrm{E}-04$ & $1.98 \mathrm{E}+07$ & $3.90 E+07$ \\
\hline 6.85 & $3.20 \mathrm{E}-04$ & $6.48 \mathrm{E}-04$ & 2.17E+07 & $4.31 E+07$ \\
\hline 7.48 & 3.49E-04 & 6.99E-04 & 2.36E+07 & $4.66 \mathrm{E}+07$ \\
\hline 8.10 & 3.77E-04 & 7.56E-04 & $2.56 \mathrm{E}+07$ & $5.05 E+07$ \\
\hline 8.72 & 4.06E-04 & $8.40 \mathrm{E}-04$ & $2.75 \mathrm{E}+07$ & $5.62 E+07$ \\
\hline 9.34 & 4.35E-04 & 8.47E-04 & $2.95 \mathrm{E}+07$ & $5.67 E+07$ \\
\hline 9.97 & $4.63 E-04$ & $8.02 \mathrm{E}-04$ & $3.14 \mathrm{E}+07$ & $5.37 \mathrm{E}+07$ \\
\hline 10.59 & 4.92E-04 & $7.68 \mathrm{E}-04$ & $3.34 \mathrm{E}+07$ & $5.14 \mathrm{E}+07$ \\
\hline 11.21 & $5.21 \mathrm{E}-04$ & 7.72E-04 & $3.53 \mathrm{E}+07$ & $5.18 \mathrm{E}+07$ \\
\hline 11.84 & 5.49E-04 & 8.36E-04 & $3.73 \mathrm{E}+07$ & $5.61 E+07$ \\
\hline 12.46 & $5.78 \mathrm{E}-04$ & 8.79E-04 & $3.92 E+07$ & $5.90 E+07$ \\
\hline 13.08 & $6.07 \mathrm{E}-04$ & $9.18 \mathrm{E}-04$ & $4.12 E+07$ & $6.17 \mathrm{E}+07$ \\
\hline 13.70 & 6.36E-04 & $9.62 E-04$ & $4.31 \mathrm{E}+07$ & $6.47 E+07$ \\
\hline 14.33 & $6.64 \mathrm{E}-04$ & $1.01 \mathrm{E}-03$ & $4.50 \mathrm{E}+07$ & $6.77 \mathrm{E}+07$ \\
\hline 14.95 & $6.93 \mathrm{E}-04$ & $1.05 \mathrm{E}-03$ & $4.70 \mathrm{E}+07$ & $7.06 \mathrm{E}+07$ \\
\hline 15.57 & $7.22 \mathrm{E}-04$ & $1.09 \mathrm{E}-03$ & $4.89 \mathrm{E}+07$ & $7.35 \mathrm{E}+07$ \\
\hline 16.20 & 7.50E-04 & 1.13E-03 & $5.09 \mathrm{E}+07$ & $7.65 E+07$ \\
\hline 16.82 & 7.79E-04 & 1.18E-03 & $5.28 \mathrm{E}+07$ & $7.96 \mathrm{E}+07$ \\
\hline 17.44 & 8.08E-04 & 1.22E-03 & $5.48 \mathrm{E}+07$ & $8.21 \mathrm{E}+07$ \\
\hline 18.07 & 8.36E-04 & $1.26 \mathrm{E}-03$ & $5.67 \mathrm{E}+07$ & $8.50 E+07$ \\
\hline 18.69 & 8.65E-04 & $1.34 \mathrm{E}-03$ & $5.87 \mathrm{E}+07$ & $9.03 E+07$ \\
\hline 19.31 & 8.94E-04 & $1.31 \mathrm{E}-03$ & $6.06 \mathrm{E}+07$ & $8.89 E+07$ \\
\hline 19.93 & $9.22 \mathrm{E}-04$ & $1.23 \mathrm{E}-03$ & $6.25 \mathrm{E}+07$ & $8.30 E+07$ \\
\hline 20.56 & 9.51E-04 & 1.16E-03 & $6.45 \mathrm{E}+07$ & $7.83 E+07$ \\
\hline 21.18 & $9.80 \mathrm{E}-04$ & $1.14 \mathrm{E}-03$ & $6.64 E+07$ & $7.72 E+07$ \\
\hline 21.80 & 1.01E-03 & $1.20 \mathrm{E}-03$ & $6.84 \mathrm{E}+07$ & $8.13 E+07$ \\
\hline 22.43 & $1.04 \mathrm{E}-03$ & $1.24 \mathrm{E}-03$ & $7.03 E+07$ & $8.36 \mathrm{E}+07$ \\
\hline 23.05 & 1.07E-03 & $1.26 \mathrm{E}-03$ & $7.23 \mathrm{E}+07$ & $8.56 \mathrm{E}+07$ \\
\hline 23.67 & 1.09E-03 & 1.30E-03 & $7.42 \mathrm{E}+07$ & $8.80 E+07$ \\
\hline 24.29 & 1.12E-03 & 1.33E-03 & $7.62 \mathrm{E}+07$ & $9.04 E+07$ \\
\hline 24.92 & 1.15E-03 & 1.37E-03 & $7.81 \mathrm{E}+07$ & $9.28 \mathrm{E}+07$ \\
\hline 25.54 & $1.18 \mathrm{E}-03$ & $1.40 \mathrm{E}-03$ & $8.01 \mathrm{E}+07$ & $9.48 E+07$ \\
\hline 26.16 & $1.21 \mathrm{E}-03$ & $1.44 \mathrm{E}-03$ & $8.20 E+07$ & $9.74 E+07$ \\
\hline 26.79 & $1.24 \mathrm{E}-03$ & $1.48 \mathrm{E}-03$ & $8.39 E+07$ & $1.01 E+08$ \\
\hline 27.41 & 1.27E-03 & $1.48 \mathrm{E}-03$ & $8.59 \mathrm{E}+07$ & $1.00 E+08$ \\
\hline 28.03 & 1.30E-03 & 1.37E-03 & $8.79 E+07$ & $9.31 E+07$ \\
\hline 28.65 & 1.32E-03 & 1.33E-03 & $8.98 \mathrm{E}+07$ & $8.99 E+07$ \\
\hline 29.28 & 1.36E-03 & 1.36E-03 & $9.24 E+07$ & $9.24 E+07$ \\
\hline 29.90 & $1.78 \mathrm{E}-16$ & 0 & -1139.4 & -4926.4 \\
\hline
\end{tabular}

Tabela 11 - Deslocamentos e Tensões - Modelo 5 - Casos 1 e 2 


\section{GRÁFICO 6 - Deslocamentos Radiais- MODELO 5 - Casos 1 e 2}

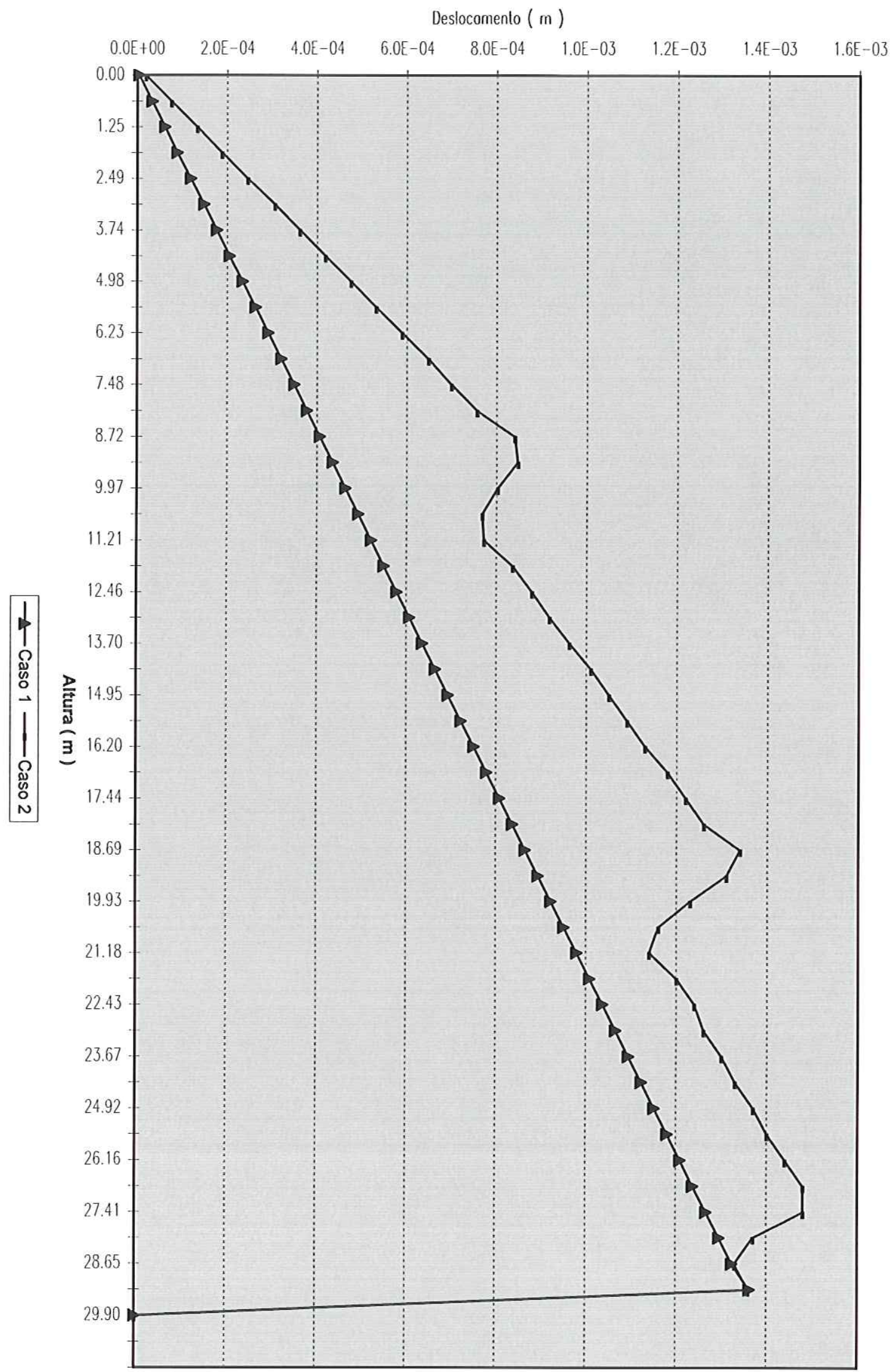


GRÁFICO 7 - Tensões Tangenciais - MODELO 5 - Casos 1 e 2

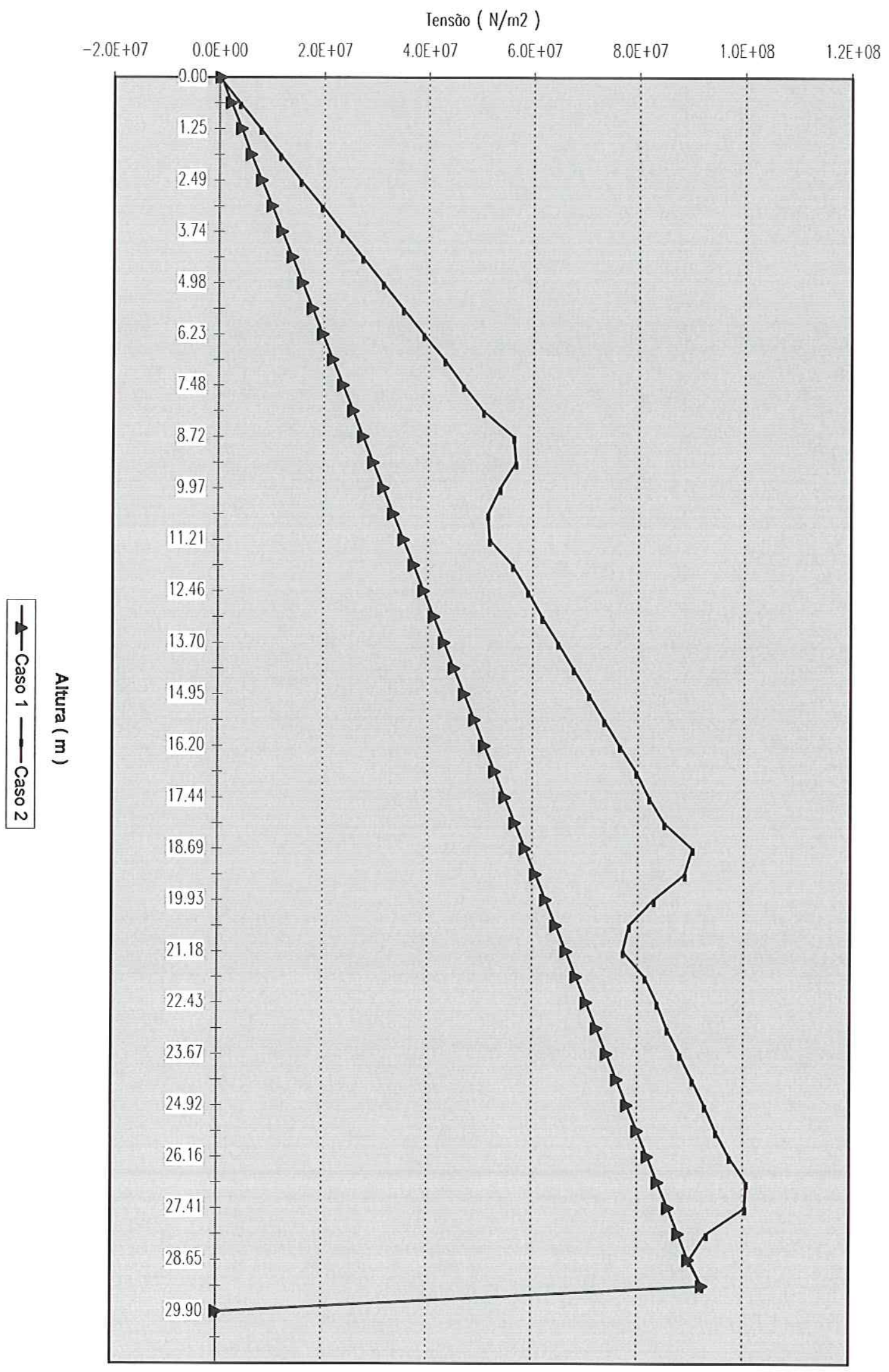


MODELO 5 - CASOS 1 e 2 - Figura 54 (Caso $3: \mathrm{e}=$ constante, Caso $4: \mathrm{e}=$ variável).
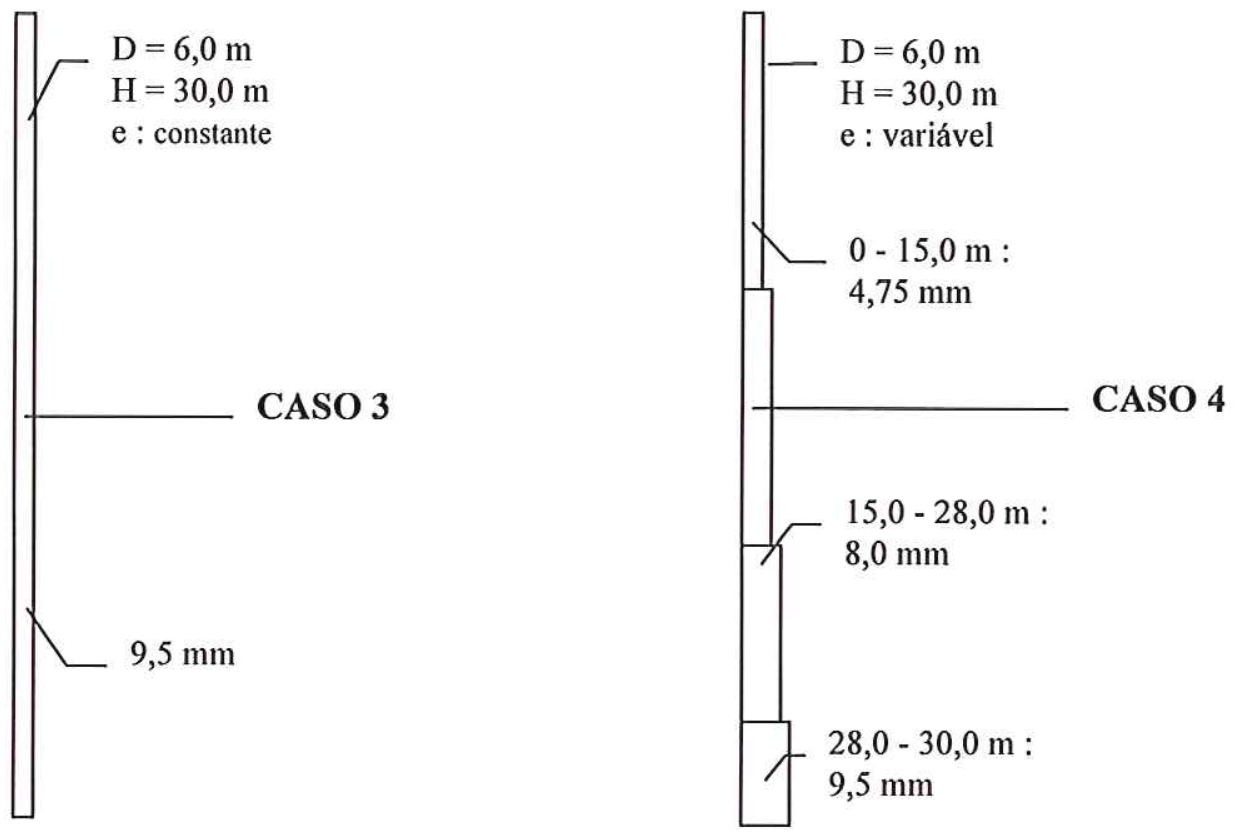

Figura 54 - Espessuras do Costado para o Modelo 5 - Casos 3 e 4.

Os deslocamentos radiais para o Caso 3, no gráfico 8, apresenta um comportamento suave, variando de valores praticamente nulos no topo, crescentes ao longo da altura, e novamente nulos no fundo da estrutura.

Os deslocamentos radiais para o Caso 4 , no gráfico 8 , apresenta um comportamento irregular, com picos de deslocamento às cotas $18,7 \mathrm{~m}, 11,20 \mathrm{~m} \mathrm{e} 8,7 \mathrm{~m}$.

As tensões tangenciais para os Casos 3 e 4, no gráfico 9, apresentam comportamentos correspondentes aos respectivos deslocamentos radiais. Um fato que não ocorreu aos outros modelos é a distribuição das tensões tangenciais para o Caso 4, da cota $7,0 \mathrm{~m}$ à cota $28,0 \mathrm{~m}$

As tensões meridionais para o Caso 3 , no gráfico 10 , apresenta valores crescentes, em uma curva suave, desde o topo até o fundo da estrutura, onde apresenta o valor máximo de compressão de membrana. Para o Caso 4 , também no gráfico 10 , ocorrem três locais de mudança de comportamento: 1 , à cota $8,72 \mathrm{~m} ; 2$, à cota $18,69 \mathrm{~m} ; 3$, à cota $27,0 \mathrm{~m}$. 
MODELO 5

CASO 3 e CASO 4

Deslocamentos e Tensões ao Longo de uma Linha Meridional

\begin{tabular}{|c|c|c|c|c|c|c|}
\hline $\bar{S}$ & $\begin{array}{c}\text { UX } \\
\text { Caso } 3 \\
\text { m }\end{array}$ & $\begin{array}{c}\text { UX } \\
\text { Caso } 4 \\
\text { m }\end{array}$ & $\begin{array}{c}\text { SY } \\
\text { Caso } 3 \\
\text { N/m2 }\end{array}$ & $\begin{array}{c}\text { SY } \\
\text { Caso } 4 \\
\text { N/m2 }\end{array}$ & $\begin{array}{c}\text { SZ } \\
\text { Caso } 3 \\
\mathrm{~N} / \mathrm{m} 2\end{array}$ & $\begin{array}{c}\text { SZ } \\
\text { Caso } 4 \\
\text { N/m2 }\end{array}$ \\
\hline 0.00 & $2.26 \mathrm{E}-05$ & 1.76E-05 & $1.54 E+06$ & 36896 & 0 & -27.379 \\
\hline 0.62 & $3.28 \mathrm{E}-05$ & 8.34E-05 & $2.22 E+06$ & $4.52 E+06$ & -17774 & -31814 \\
\hline 1.25 & $6.60 \mathrm{E}-05$ & $1.51 \mathrm{E}-04$ & $4.45 E+06$ & $9.14 E+06$ & -75361 & $-1.24 E+05$ \\
\hline 1.87 & 7.93E-05 & $1.59 E-04$ & $5.32 E+06$ & $9.62 E+06$ & $-1.80 E+05$ & $-3.23 E+05$ \\
\hline 2.49 & 9.57E-05 & 2.03E-04 & $6.40 E+06$ & $1.25 E+07$ & $-2.98 E+05$ & $-5.65 E+05$ \\
\hline 3.11 & $1.11 \mathrm{E}-04$ & $2.32 \mathrm{E}-04$ & $7.39 E+06$ & $1.45 \mathrm{E}+07$ & $-4.46 E+05$ & $-8.37 E+05$ \\
\hline 3.74 & 1.27E-04 & $2.64 \mathrm{E}-04$ & $8.43 E+06$ & $1.66 \mathrm{E}+07$ & $-6.49 E+05$ & $-1.24 E+06$ \\
\hline 4.36 & $1.42 \mathrm{E}-04$ & $2.94 \mathrm{E}-04$ & $9.37 E+06$ & $1.85 E+07$ & $-8.93 E+05$ & $-1.72 E+06$ \\
\hline 4.98 & $1.56 \mathrm{E}-04$ & $3.22 \mathrm{E}-04$ & $1.03 E+07$ & $2.03 E+07$ & $-1.16 \mathrm{E}+06$ & $-2.24 \mathrm{E}+06$ \\
\hline 5.61 & $1.70 \mathrm{E}-04$ & $3.48 \mathrm{E}-04$ & $1.11 \mathrm{E}+07$ & $2.19 \mathrm{E}+07$ & $-1.47 E+06$ & $-2.86 E+06$ \\
\hline 6.23 & 1.83E-04 & $3.74 \mathrm{E}-04$ & $1.19 \mathrm{E}+07$ & $2.35 \mathrm{E}+07$ & $-1.80 E+06$ & $-3.52 E+06$ \\
\hline 6.85 & 1.95E-04 & $4.00 \mathrm{E}-04$ & $1.26 E+07$ & $2.51 \mathrm{E}+07$ & $-2.15 E+06$ & $-4.23 E+06$ \\
\hline 7.48 & 2.07E-04 & $4.20 \mathrm{E}-04$ & $1.33 E+07$ & $2.63 E+07$ & $-2.55 E+06$ & $-5.01 E+06$ \\
\hline 8.10 & $2.18 \mathrm{E}-04$ & 4.43E-04 & $1.39 \mathrm{E}+07$ & $2.76 \mathrm{E}+07$ & $-2.95 E+06$ & $-5.81 E+06$ \\
\hline 8.72 & 2.29E-04 & 4.79E-04 & $1.45 E+07$ & $2.98 \mathrm{E}+07$ & $-3.39 E+06$ & $-6.68 E+06$ \\
\hline 9.34 & $2.39 \mathrm{E}-04$ & $4.72 \mathrm{E}-04$ & $1.51 \mathrm{E}+07$ & $2.92 \mathrm{E}+07$ & $-3.84 E+06$ & $-7.16 E+06$ \\
\hline 9.97 & $2.50 \mathrm{E}-04$ & 4.37E-04 & $1.56 \mathrm{E}+07$ & $2.68 \mathrm{E}+07$ & $-4.31 E+06$ & $-7.38 E+06$ \\
\hline 10.59 & $2.59 \mathrm{E}-04$ & $4.09 E-04$ & $1.61 \mathrm{E}+07$ & $2.49 \mathrm{E}+07$ & $-4.81 E+06$ & $-7.54 E+06$ \\
\hline 11.21 & 2.68E-04 & 4.02E-04 & $1.66 \mathrm{E}+07$ & $2.43 E+07$ & $-5.32 E+06$ & $-7.95 E+06$ \\
\hline 11.84 & 2.77E-04 & $4.26 \mathrm{E}-04$ & $1.71 E+07$ & $2.58 \mathrm{E}+07$ & $-5.84 E+06$ & $-8.75 E+06$ \\
\hline 12.46 & $2.86 \mathrm{E}-04$ & 4.39E-04 & $1.75 E+07$ & $2.64 E+07$ & $-6.39 E+06$ & $-9.57 E+06$ \\
\hline 13.08 & $2.94 \mathrm{E}-04$ & 4.49E-04 & $1.79 E+07$ & $2.68 \mathrm{E}+07$ & $-6.95 E+06$ & $-1.04 E+07$ \\
\hline 13.70 & $3.02 E-04$ & 4.61E-04 & $1.82 E+07$ & $2.74 \mathrm{E}+07$ & $-7.52 E+06$ & $-1.13 E+07$ \\
\hline 14.33 & $3.10 \mathrm{E}-04$ & 4.73E-04 & $1.86 E+07$ & $2.80 E+07$ & $-8.11 E+06$ & $-1.22 E+07$ \\
\hline 14.95 & $3.17 \mathrm{E}-04$ & $4.83 \mathrm{E}-04$ & $1.89 \mathrm{E}+07$ & $2.85 \mathrm{E}+07$ & $-8.70 E+06$ & $-1.31 E+07$ \\
\hline 15.57 & $3.24 \mathrm{E}-04$ & $4.94 \mathrm{E}-04$ & $1.92 E+07$ & $2.89 \mathrm{E}+07$ & $-9.31 E+06$ & $-1.40 E+07$ \\
\hline 16.20 & 3.31E-04 & $5.04 \mathrm{E}-04$ & $1.95 E+07$ & $2.94 E+07$ & $-9.93 E+06$ & $-1.49 E+07$ \\
\hline 16.82 & 3.38E-04 & $5.15 E-04$ & $1.98 E+07$ & $2.99 E+07$ & $-1.06 E+07$ & $-1.59 E+07$ \\
\hline 17.44 & $3.44 \mathrm{E}-04$ & 5.22E-04 & $2.00 E+07$ & $3.01 \mathrm{E}+07$ & $-1.12 E+07$ & $-1.68 E+07$ \\
\hline 18.07 & $3.51 \mathrm{E}-04$ & 5.31E-04 & $2.03 E+07$ & $3.04 \mathrm{E}+07$ & $-1.18 E+07$ & $-1.78 E+07$ \\
\hline 18.69 & $3.57 \mathrm{E}-04$ & $5.54 \mathrm{E}-04$ & $2.05 E+07$ & $3.17 E+07$ & $-1.25 E+07$ & $-1.88 E+07$ \\
\hline 19.31 & $3.63 E-04$ & $5.37 \mathrm{E}-04$ & $2.07 E+07$ & $3.05 E+07$ & $-1.32 E+07$ & $-1.89 E+07$ \\
\hline 19.93 & 3.69E-04 & $4.93 E-04$ & $2.09 E+07$ & $2.77 \mathrm{E}+07$ & $-1.38 E+07$ & $-1.85 E+07$ \\
\hline 20.56 & $3.75 \mathrm{E}-04$ & $4.58 \mathrm{E}-04$ & $2.11 \mathrm{E}+07$ & $2.55 \mathrm{E}+07$ & $-1.45 E+07$ & $-1.80 E+07$ \\
\hline 21.18 & 3.80E-04 & $4.44 \mathrm{E}-04$ & 2.13E+07 & $2.46 \mathrm{E}+07$ & $-1.52 E+07$ & $-1.80 E+07$ \\
\hline 21.80 & 3.86E-04 & 4.62E-04 & $2.14 E+07$ & $2.55 E+07$ & $-1.59 E+07$ & $-1.88 E+07$ \\
\hline 22.43 & 3.91E-04 & $4.68 \mathrm{E}-04$ & $2.16 \mathrm{E}+07$ & $2.57 E+07$ & $-1.66 E+07$ & $-1.97 E+07$ \\
\hline 23.05 & 3.96E-04 & 4.71E-04 & $2.17 E+07$ & $2.57 E+07$ & $-1.73 E+07$ & $-2.05 E+07$ \\
\hline 23.67 & 4.01E-04 & $4.78 \mathrm{E}-04$ & $2.19 \mathrm{E}+07$ & $2.59 E+07$ & $-1.80 E+07$ & $-2.14 E+07$ \\
\hline 24.29 & 4.06E-04 & 4.84E-04 & $2.20 E+07$ & $2.61 \mathrm{E}+07$ & $-1.87 E+07$ & $-2.22 E+07$ \\
\hline 24.92 & 4.11E-04 & $4.90 \mathrm{E}-04$ & $2.21 \mathrm{E}+07$ & $2.63 E+07$ & $-1.94 E+07$ & $-2.31 E+07$ \\
\hline 25.54 & 4.16E-04 & 4.94E-04 & $2.22 \mathrm{E}+07$ & $2.63 E+07$ & $-2.01 E+07$ & $-2.39 E+07$ \\
\hline 26.16 & 4.21E-04 & 5.01E-04 & $2.23 E+07$ & $2.65 E+07$ & $-2.09 E+07$ & $-2.48 E+07$ \\
\hline 26.79 & 4.26E-04 & 5.11E-04 & $2.24 \mathrm{E}+07$ & $2.70 E+07$ & $-2.16 E+07$ & $-2.57 E+07$ \\
\hline 27.41 & 4.30E-04 & 5.03E-04 & $2.25 E+07$ & $2.65 E+07$ & $-2.23 E+07$ & $-2.54 E+07$ \\
\hline 28.03 & 4.35E-04 & 4.62E-04 & $2.26 \mathrm{E}+07$ & $2.41 E+07$ & $-2.32 E+07$ & $-2.43 E+07$ \\
\hline 28.65 & 4.40E-04 & 4.41E-04 & $2.26 \mathrm{E}+07$ & $2.27 E+07$ & $-2.41 E+07$ & $-2.41 E+07$ \\
\hline 29.28 & 4.49E-04 & 4.49E-04 & $2.30 E+07$ & $2.30 E+07$ & $-2.50 E+07$ & $-2.50 E+07$ \\
\hline 29.90 & $5.81 \mathrm{E}-17$ & 0 & $-7.62 E+06$ & $-7.62 E+06$ & $-2.56 E+07$ & $-2.56 E+07$ \\
\hline
\end{tabular}




\section{GRÁFICO 8 - Deslocamentos Radiais- MODELO 5 - Casos 3 e 4}

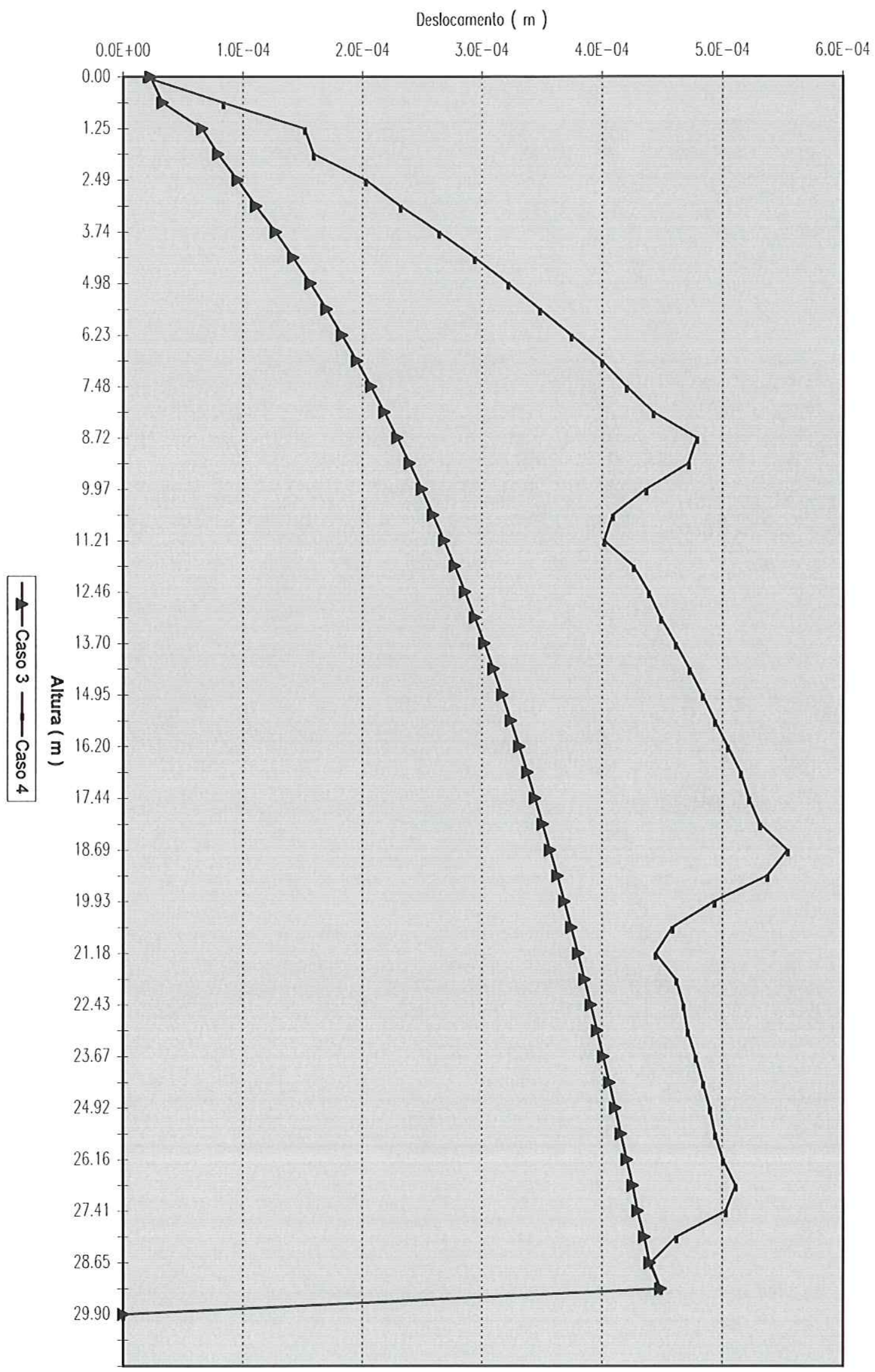


GRÁFICO 9 - Tensões Tangenciais - MODELO 5 - Casos 3 e 4

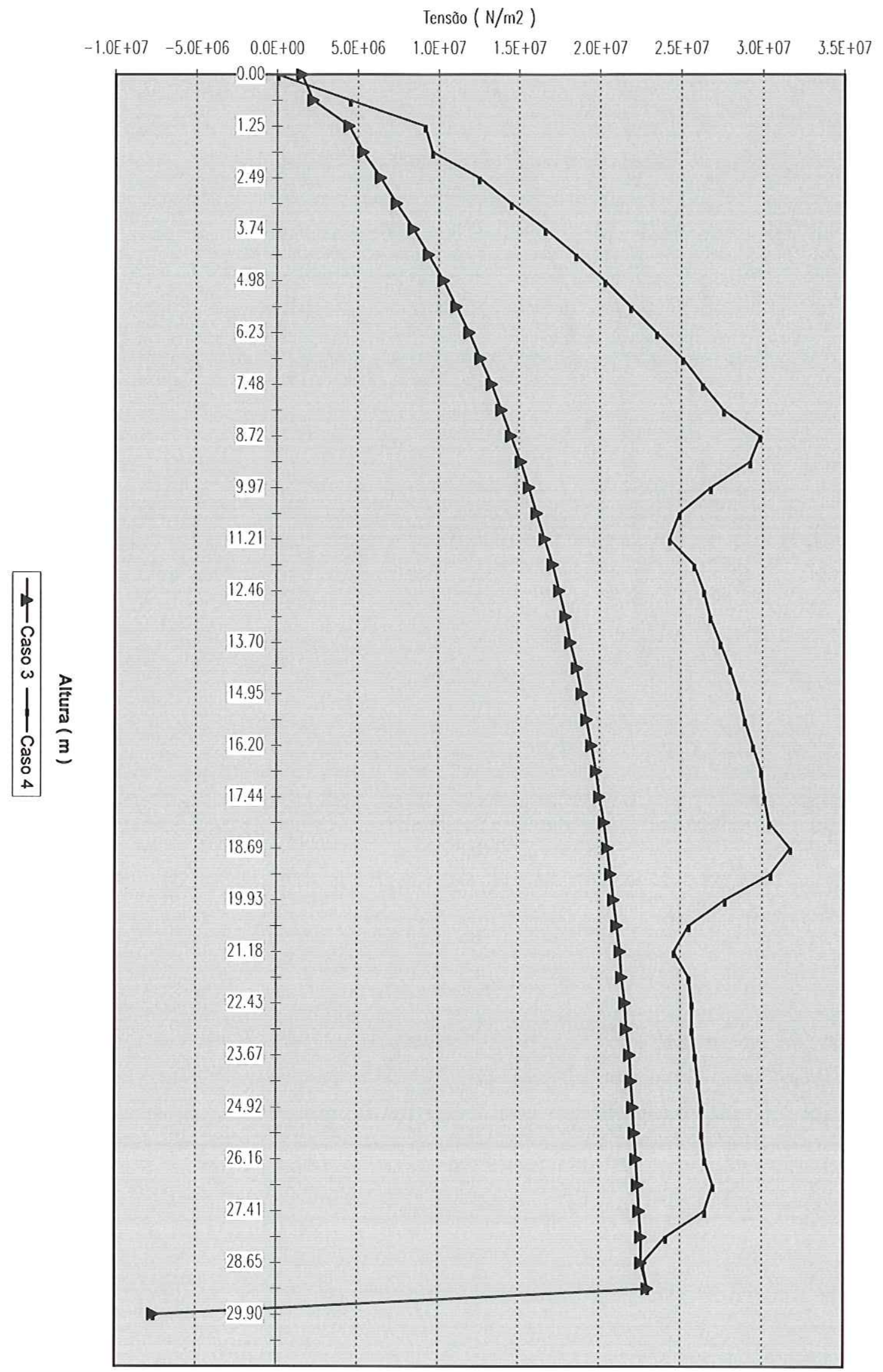




\section{GRÁFICO 10 - Tensões Meridionais - MODELO 5 - Casos 3 e 4}

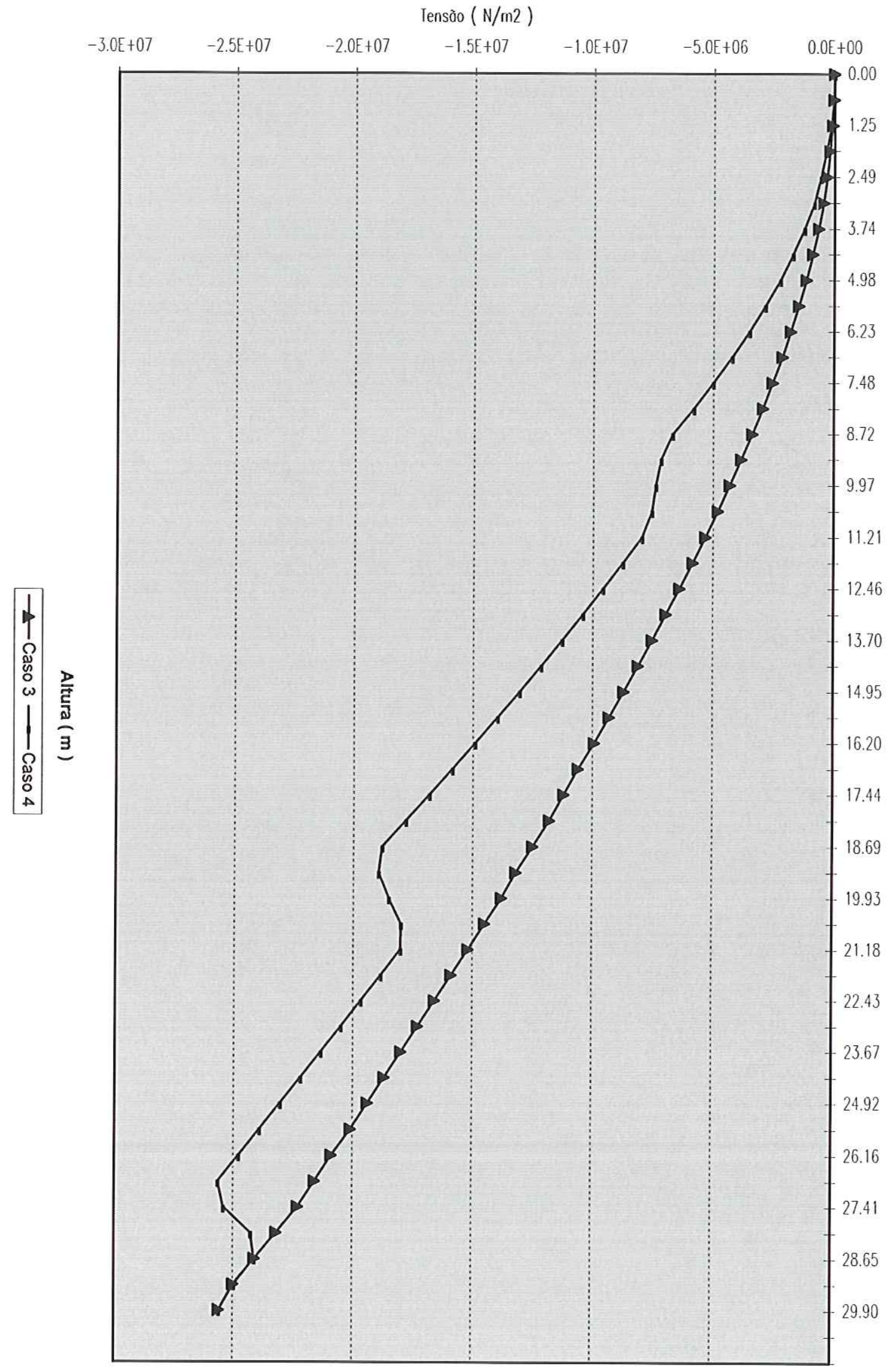


MODELO 5 - CASOS 5 e 6 -Fig 55 (Caso 5) e Fig 56(Caso 6) Tensões tangenciais em Pa
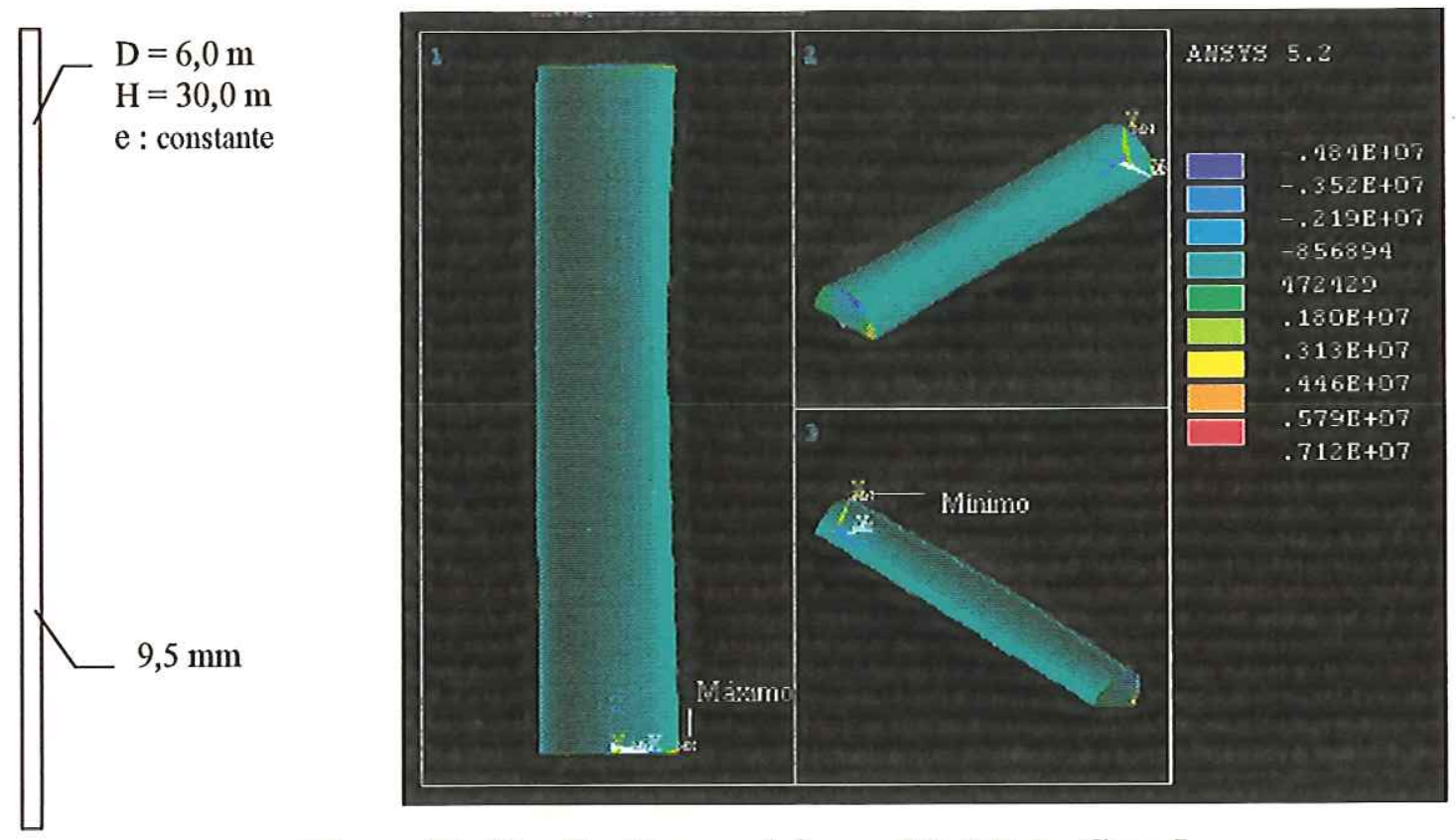

Figura 55 - Tensões Tangenciais $\sigma_{y}$ - Modelo 5 - Caso 5
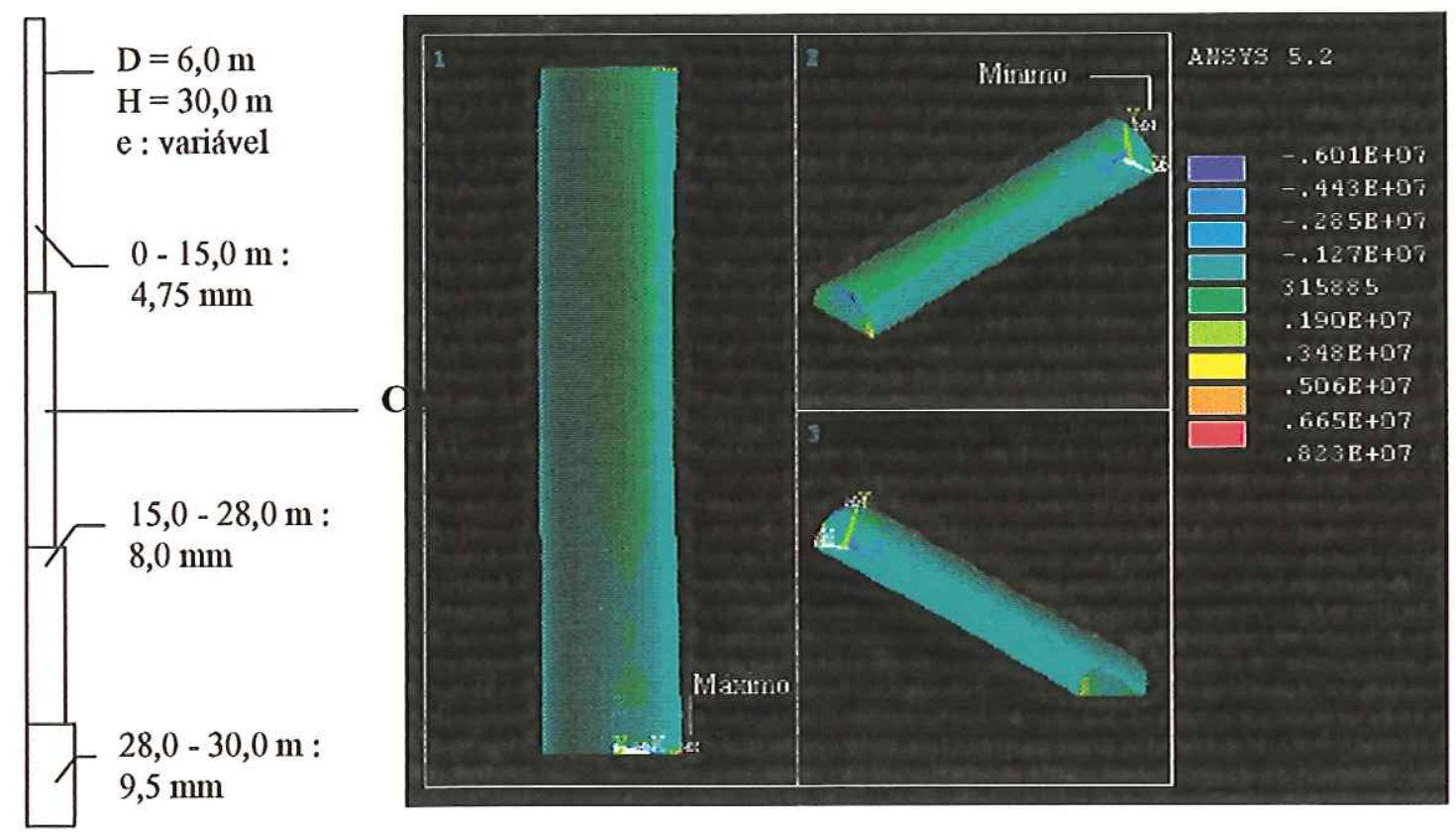

Figura 56 - Tensões Tangenciais $\sigma_{y}$ - Modelo 5 - Caso 6

As tensões tangenciais no Caso 5, figura 55, apresentam uma distribuição onde não se destacam regiões de concentração de tensões, mas é possível distinguir que as maiores tensões estão localizadas próximo ao topo. As tensões tangenciais para oCaso 6, figura 56, têm uma distribuição mais predominante em verde-claro ( tensões de sinal positivo, ou de tração) logo abaixo da região em azul ( na tampa ). 
MODELO 5 - Fig. 57 (Caso 5) e Fig. 58 (Caso 6) Tensões meridionais em Pa
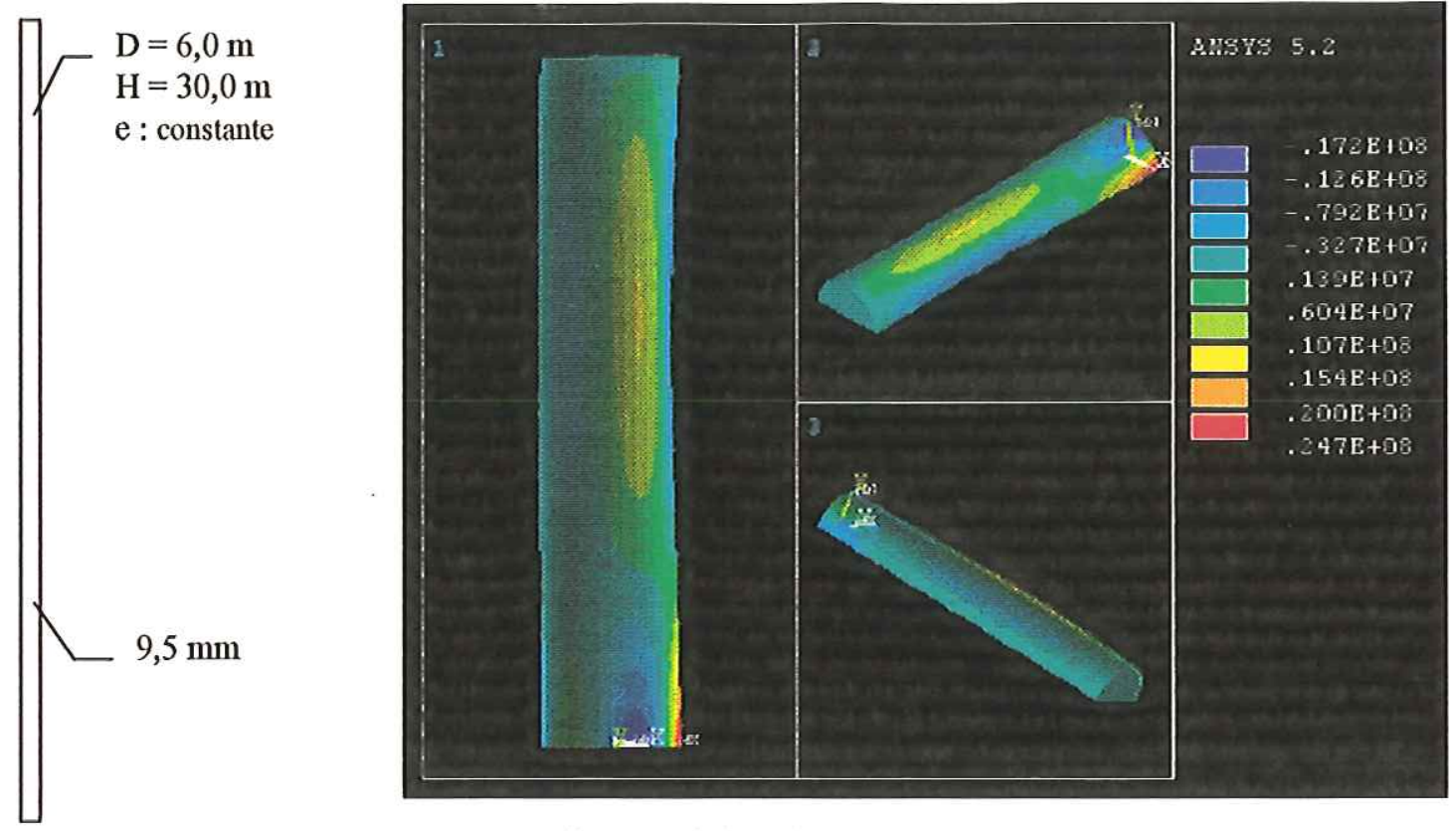

Figura 57- Tensões Meridionais $\sigma_{\mathrm{Z}}$ - Modelo 5 - Caso 5
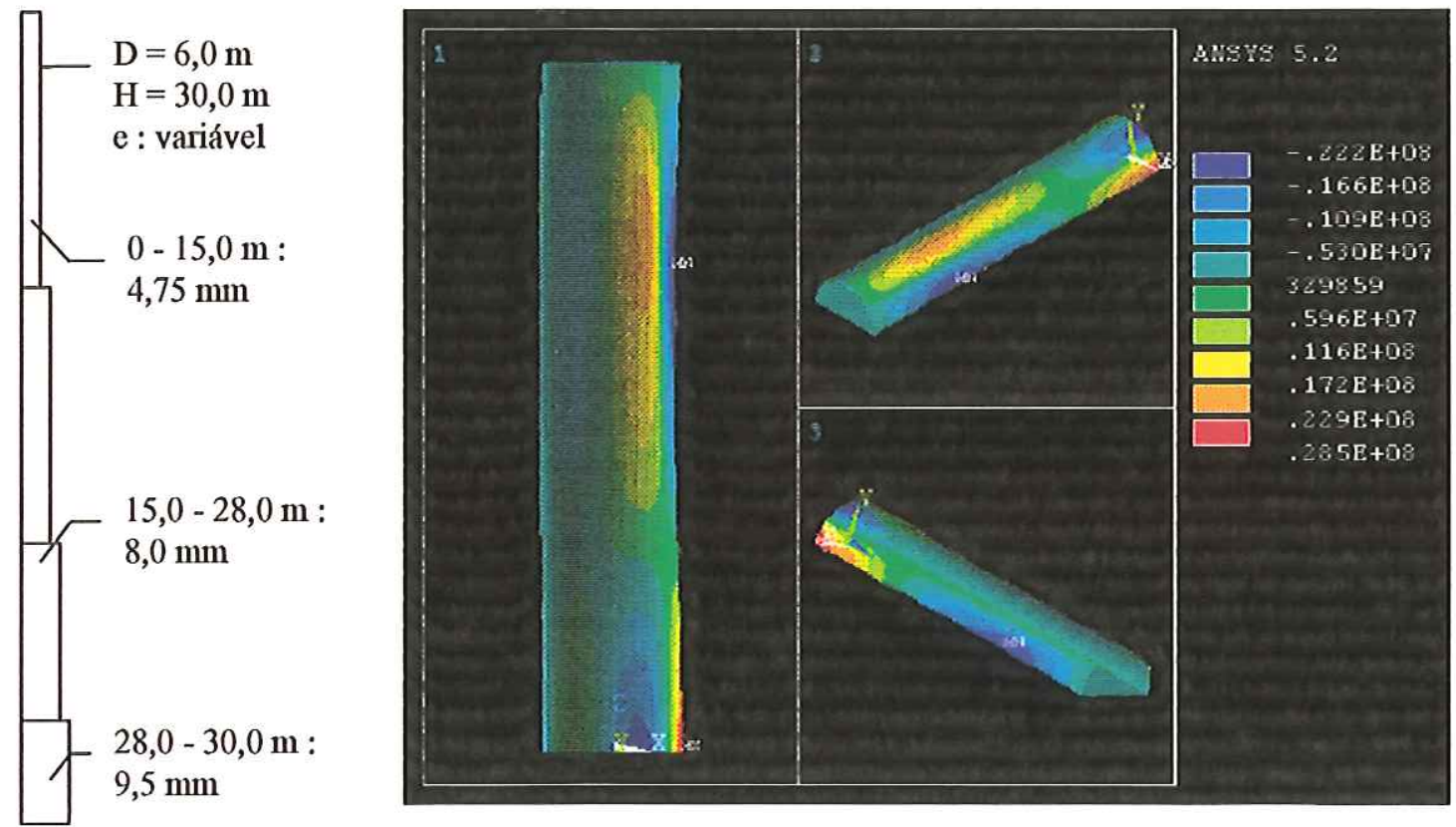

Figura 58 - Tensões Meridionais $\sigma_{\mathrm{Z}}-$ Modelo 5 - Caso 6

As tensões meridionais para o Caso 5, figura 57, têm valores de compressão elevados nas duas regiões em azul, devendo-se avaliar as duas partes para o fenômeno de flambagem. Já as tensões meridionais de tração são mais pronunciadas junto à base. 
Para o Caso 6, figura 58, as tensões de compressão têm os maiores valores nas duas regiões em azul, devendo se avaliar o fenômeno de flambagem em ambas. As tensões de tração se destacam nas regiões em tons alaranjados próximas às extremidades da estrutura.

Quando comparados entre si, os Casos 5 e 6 mostram que as tensões de compressão do Caso 6 têm seus valores aumentados significativamente, em relação aos valores do Caso 5 , para as regiões próximas às extremidades da estrutura.

\subsection{2 - Comparação das Regiões de tensão entre os Modelos.}

O objetivo é avaliar as regiões de concentração das tensões tangenciais e das tensões meridionais, comparando-se os casos 3 e 4 (solicitação do material sólido) e os casos 5 e 6 (vento) dos 5 modelos simulados.

A abordagem consiste em distinguir e mostrar:

1. O posicionamento das regiões de concentração de tensões em cada caso $(3,4)$ e $(5,6)$;

2. O tipo de tensão (meridional, tangencial, compressão ou tração) associado à região;

3. Onde ocorrem os picos de tensão;

4. Os maiores valores.

Para as tensões tangenciais, nos casos 3 e 4, ocorrem:

- Em todos os modelos as tensões são crescentes no sentido do topo para a base do silo, onde atingem os maiores valores absolutos;

- Os picos de tensões ocorrem quando há variação de espessura das chapas metálicas;

- próximo ao fundo do silo, sendo a espessura igual para todos os modelos, a variação das tensões segue um mesmo comportamento;

- A distribuição é axissimétrica, já que a saída do material ensilado é centrada no fundo da estrutura;

- Predominam as tensões de tração do topo até próximo à base, onde podem ocorrer tensões de compressão tangencial.

Para as tensões meridionais nos casos de material ensilado ( $\operatorname{casos} 3$ e 4 ):

- Apenas ocorrem tensões de compressão;

- As tensões de compressão são crescentes (em valores absolutos) no sentido do topo para o fundo do silo; 
- Os picos de tensão de compressão ocorrem onde há encontro de chapas metálicas de espessuras diferentes.

Para a solicitação do vento (casos 5 e 6), as tensões tangenciais são:

- Quanto maior a relação altura/diâmetro (H/D), menores ficam as regiões de concentração de tensões tangenciais;

- Quanto maior a relação diâmetro/espessura, também são maiores os valores das tensões tangenciais;

- As regiões de concentração de tensão localizam-se na metade superior da estrutura cilíndrica;

- As regiões de tração estão localizadas entre as áreas do silo a barlavento e a sotavento, na direção perpendicular à ação do vento;

- Os maiores valores das tensões ocorrem a barlavento;

Comparando as tensões meridionais para a solicitação de vento (casos 5 e 6):

- As duas regiões que mais concentram tensões de compressão localizam-se: 1) a barlavento, próximo ao topo e a $40^{\circ}$ (medidos a partir da direção do vento); 2) próxima à base. Ocorre ainda uma outra região de compressão a sotavento, com menores valores que as tensões a barlavento.

- Quanto maior a relação altura/diâmetro (H/D), maiores são os valores das tensões localizados junto à base da estrutura. 


\section{CAPÍTULO 5 - Caso Exemplo: Silo de Grãos.}

O exemplo é para um reservatório cilíndrico de fundo plano, apoiado diretamente ao nível do solo, de volume aproximado $1615 \mathrm{~m}^{3}$ (Altura $\mathrm{H}=17 \mathrm{~m}$, diâmetro $\mathrm{D}=11 \mathrm{~m}$ ).

\section{Roteiro}

a) Determinação das pressões que atuam no silo:

- a .1) Tampa : pressão do vento

- a .2) Parede: pressão devida ao material armazenado pressão do vento

- a .3) Fundo: pressão devida ao material armazenado.

Normas técnicas utilizadas:

- NBR6123 - Forças devidas ao vento em edificações

- DIN1055-6 - Hipóteses para ações de projeto para construções, ações em silos (Lastannahmen für Bauten, Lasten in Silozellen).

- DIN 18914 - Silos cilíndricos elevados de paredes delgadas em aço. Düsseldorf, set 1985

b) Determinação das espessuras das chapas.

- b.1) Tampa

- $\quad$ .2) Parede

- 1) Combinação das ações

- 2) Determinação das espessuras das chapas

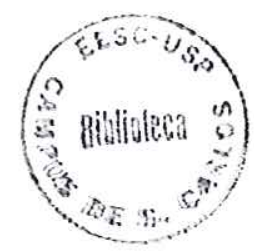


a) Determinação das pressões que atuam no silo.

a.1 - Determinação das pressões que atuam na tampa.

- Pressão do vento em tampa cônica

Os coeficiente de pressão externa $\mathrm{C}_{\text {pe }}$ são distribuídos em faixas (coeficientes extraídos de Briassoulis ( 1986)):

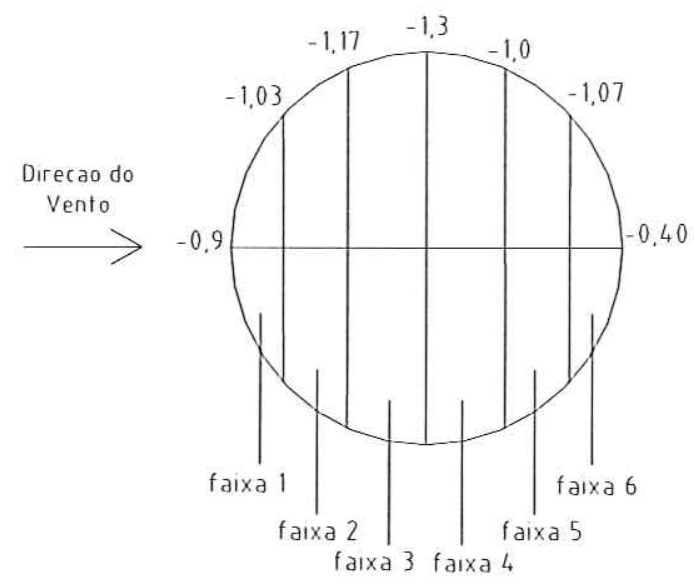

Figura 59 - Faixas de Coeficientes de Pressão em Cobertura Cônica

Velocidade básica adotada: $\quad \mathrm{V}_{0}=40 \mathrm{~m} / \mathrm{s}$

$$
\begin{array}{rlr}
\text { fatores adotados: } & \mathrm{S}_{1}=1,0 & \text { (fator topográfico) } \\
& \mathrm{S}_{2}=0,94 .(\mathrm{z} / 10)^{0,105}=0,94 .(17 / 10)^{0,105} \\
& \mathrm{~S}_{2}=0,99 \quad & \text { (terreno com pequenos obstáculos) } \\
\mathrm{S}_{3}=0,95 & \text { (Baixo fator de ocupação humana) }
\end{array}
$$

$\Delta \mathrm{p}$ em Newtons $/ \mathrm{m}^{2}$ (componente estática de pressão do vento que atua perpendicularmente sobre um elemento de área)

$$
\begin{aligned}
& \Delta \mathrm{p}=\mathrm{C}_{\mathrm{pe}} \cdot \mathrm{q} \cdot \mathrm{dA} \\
& \mathrm{q}=0,613 \cdot \mathrm{v}_{\mathrm{k}}{ }^{2}\left(\mathrm{~N} / \mathrm{m}^{2}\right) \\
& \mathrm{V}_{\mathrm{k}}=\mathrm{S}_{1} \cdot \mathrm{S}_{2} \cdot \mathrm{S}_{3} \cdot \mathrm{V}_{0}=1,0.0,99 \cdot 0,95 \cdot 40 \\
& \mathrm{~V}_{\mathrm{k}}=37,62 \mathrm{~m} / \mathrm{s} \\
& \text { Portanto, } \quad q=867,56 \mathrm{~N} / \mathrm{m}^{2}
\end{aligned}
$$




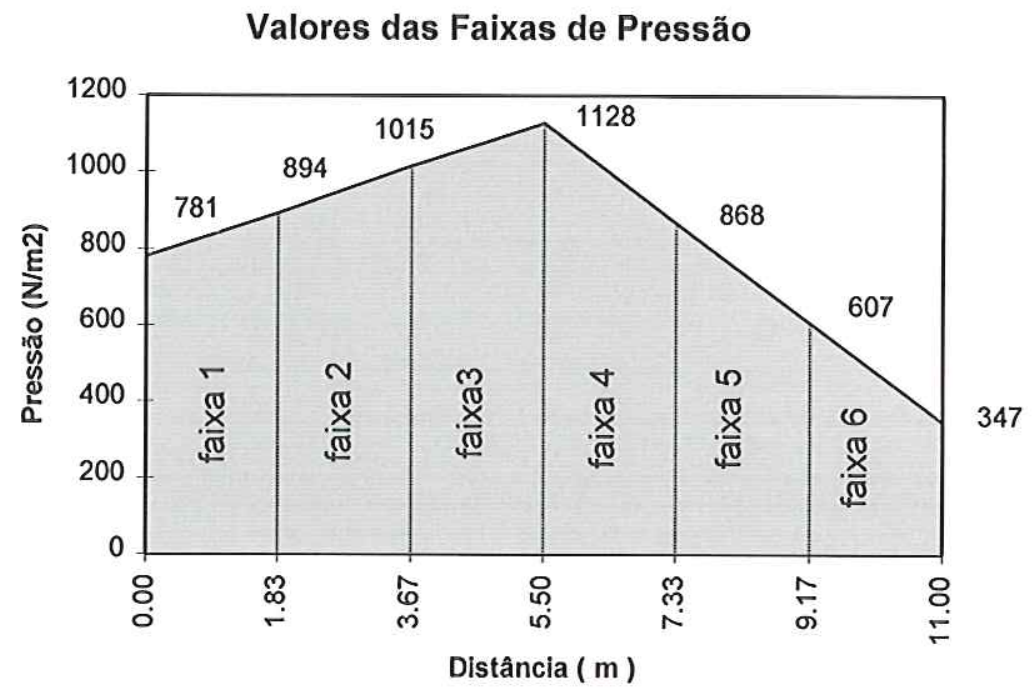

Figura 60 - Valores das Pressões na cobertura do silo exemplo

$\mathrm{O}$ cálculo das áreas da cobertura para as faixas 1 a 6 está indicado no Apêndice - Cálculo das Áreas de Fatias da Superficie Cônica. Os resultados são:

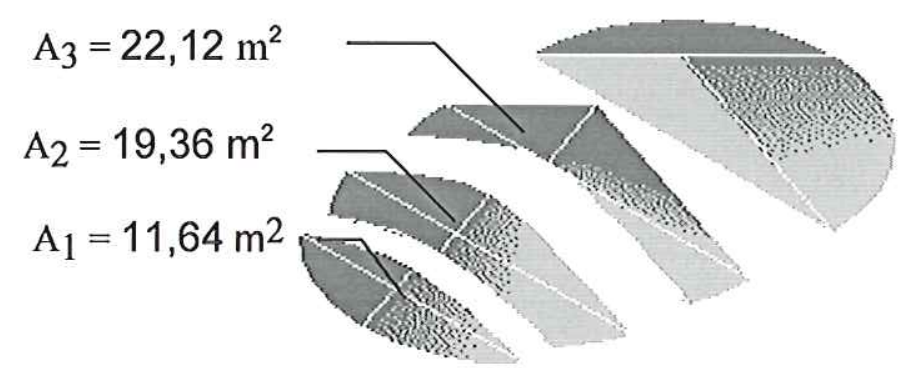

Figura 61 - Áreas das Faixas de Pressão.

Com os valores da pressão do vento e as áreas onde atua o vento, pode-se calcular os esforços que a cobertura transmite ao costado do silo: 


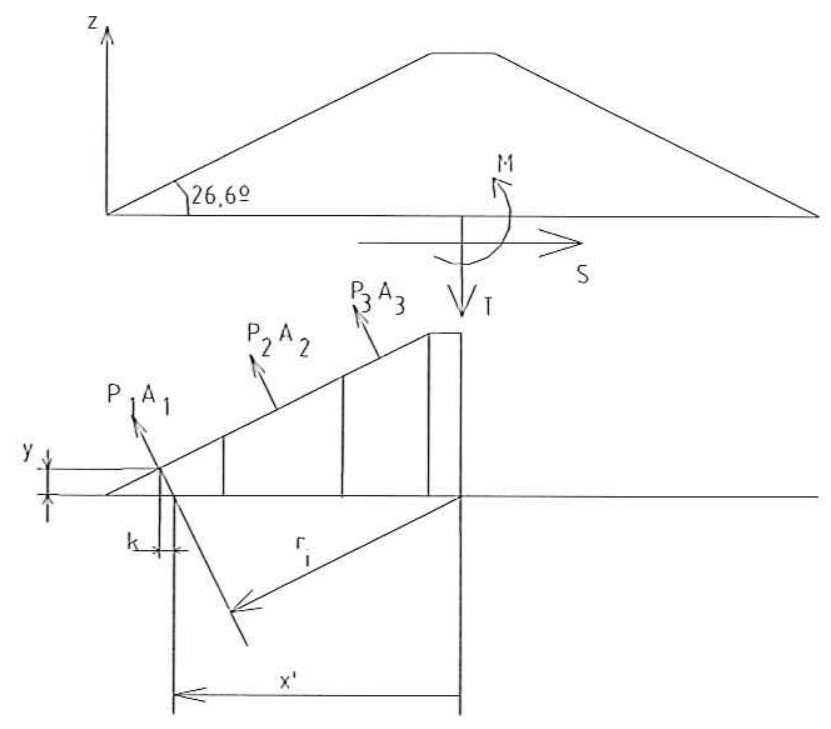

Figura 62 - Esforços transmitidos pela tampa ao costado do Silo.

\section{Equilíbrio:}

\section{Direção Z:}

$$
-T+\left(\sum_{1}^{6} P_{i} A_{i}\right) \cdot \cos 26,6^{\circ}=0 \quad T=83.914 N
$$

\section{Direção X:}

$$
\begin{aligned}
& S+\left(-P_{1} A_{1}-P_{2} A_{2}-P_{3} A_{3}\right) \cdot \operatorname{sen}\left(26,6^{\circ}\right)+\left(P_{4} A_{4}+5_{5} A_{5}+P_{6} A_{6}\right) \cdot \operatorname{sen}\left(26,6^{\circ}\right)=0 \\
& S=8.963 N
\end{aligned}
$$

Momento em torno do eixo central y:

$$
\begin{aligned}
& M+P_{1} A_{1} r_{1}+P_{2} A_{2} r_{2}+P_{3} A_{3} r_{3}+P_{4} A_{4} r_{4}+P_{5} A_{5} r_{5}+P_{5} A_{6} r_{6}=0 \quad r_{i}=x^{\prime} \cos 26,6^{\circ} \\
& M=23.804 \mathrm{~N} \cdot \mathrm{m}
\end{aligned}
$$

a .2) Determinação das Pressões que atuam na parede do silo

a . 2.1) Pressão devida ao vento

Extraindo os coeficientes da Tabela 1 (pág. 20), monta-se a Tabela 13 - Pressões do Vento ao longo da altura e do perímetro: 
Tabela 13 - Pressões do Vento ao Longo da Altura e do Perímetro

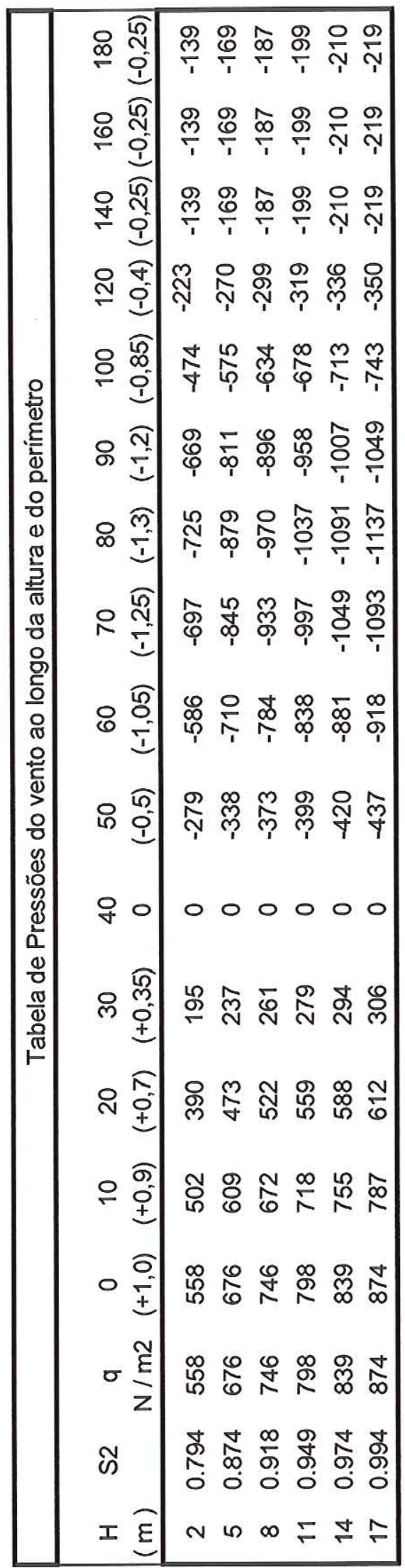

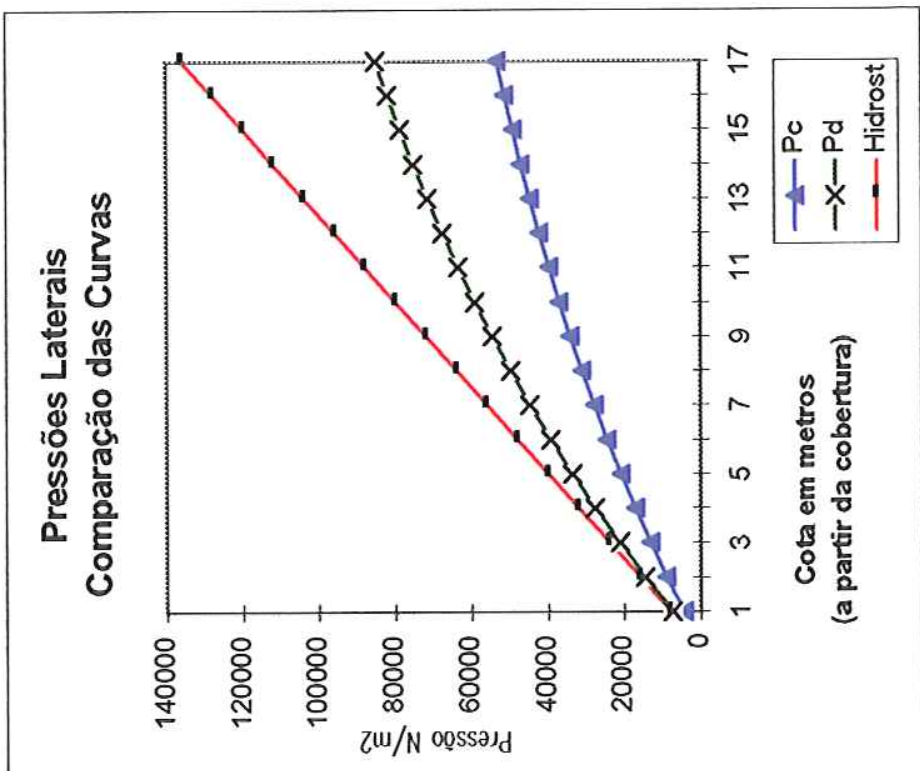

1

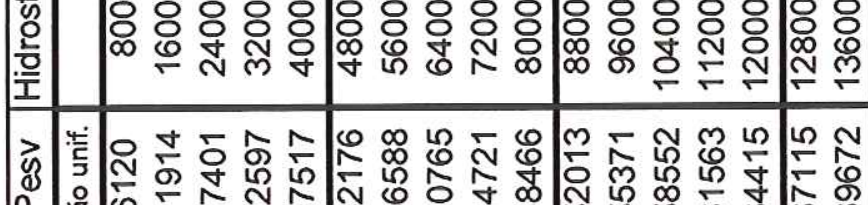
Q

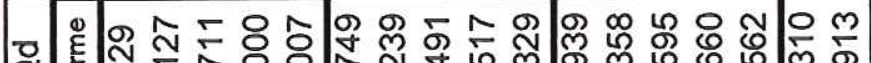
O

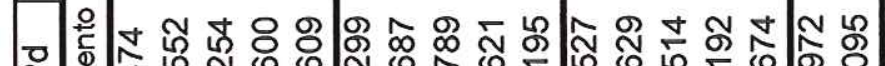

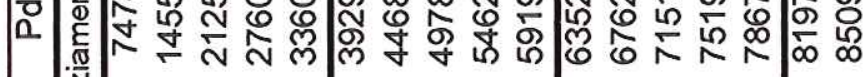
윰

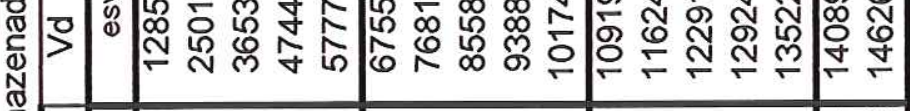

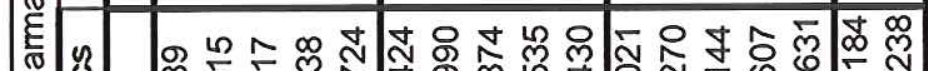

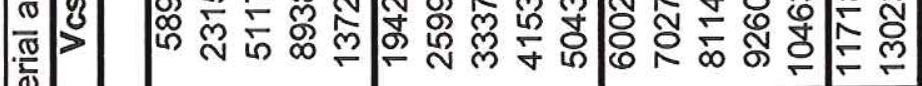

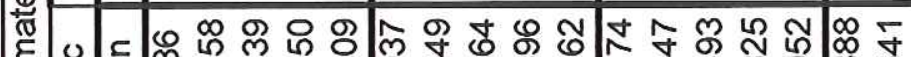
定 疍

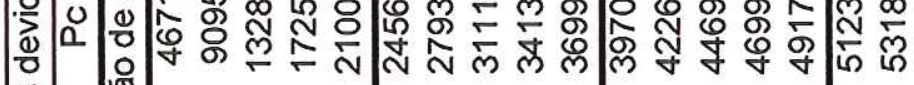
造

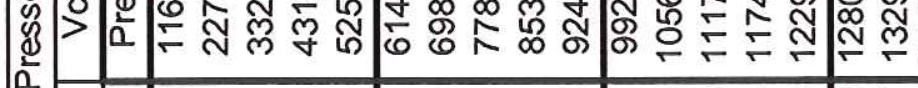

\begin{tabular}{|c|c|c|c|}
\hline & 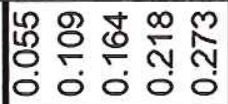 & 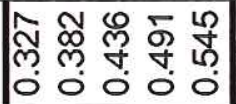 & 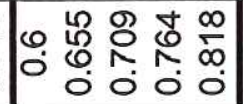 \\
\hline$\varepsilon$ & $m+\infty$ & on $\infty$ 음 & $E \cong \stackrel{M}{ }=\underline{L}$ \\
\hline
\end{tabular}


a .2.2) Pressão Hidrostática

A pressão hidrostática $\mathrm{p}$, em função da profundidade $\mathrm{z}, \mathrm{p}(\mathrm{z})$, é calculada pela relação:

$$
p=\gamma . z \quad p=10000 * 17,0 \quad p=170.000 N / m^{2}
$$

a .2.3) Pressão devida ao material armazenado - calculada com a DIN 1055-6 (1986)

- Material: milho

- $\gamma=8000 \mathrm{~N} / \mathrm{m}^{3} \quad$ peso específico

- $\phi=28^{\circ} \quad$ ângulo de atrito entre os grãos

- $\mu^{\prime}=0,25 \quad$ coeficiente de atrito entre os grãos e a parede

- $\mu=0,53 \quad \mu=\operatorname{tg} \phi$

\begin{tabular}{|c|c|c|}
\hline Perímetro $\mathrm{U}$ & $\begin{array}{c}\text { Área da } \\
\text { Seção }\end{array}$ & Raio hidráulico da célula cilíndrica \\
\hline $\begin{array}{c}\mathrm{U}=3,14 . \mathrm{D} \\
\mathrm{U}=34,56 \mathrm{~m}\end{array}$ & $\mathrm{~S}=95,03 \mathrm{~m}^{2}$ & $\begin{array}{c}\mathrm{S} / \mathrm{U}=2,75 \mathrm{~m} \\
\text { (raio hidráulico da célula cilíndrica) }\end{array}$ \\
\hline
\end{tabular}

Para o caso de esvaziamento não-uniforme: $\mathrm{p}_{\mathrm{c}}=\mathrm{C}_{\mathrm{m}}$. (Pressão estática de Janssen)

São também considerados os impactos devido ao possível desmoronamento de cúpulas. Para esses casos, os coeficientes adotados são:

\begin{tabular}{|c|c|c|c|c|c|c|}
\hline $\begin{array}{c}\mathrm{C}=\mathrm{C}_{\mathrm{h}} \mathrm{C}_{\mathrm{e}} \mathrm{C}_{\mathrm{r}} \\
\mathrm{C}_{\mathrm{g}}\end{array}$ & $\begin{array}{c}\mathrm{C}_{\mathrm{h}}= \\
0,13 * \mathrm{H} / \mathrm{D}+.87\end{array}$ & $\mathrm{C}_{\mathrm{e}}$ & $\mathrm{C}_{\mathrm{r}}$ & $\mathrm{C}_{\mathrm{g}}$ & $\begin{array}{c}\mathrm{C}_{\mathrm{m}}= \\
1+1,3 \mathrm{C} \sqrt{17 / 11}\end{array}$ & $\begin{array}{c}\mathrm{C}_{\mathrm{S}}= \\
1+0,5 / \sqrt{5,5}\end{array}$ \\
\hline 0,048 & 1,07 & 1,0 & 0,05 & 0,9 & 1,08 & 1,21 \\
\hline
\end{tabular}

a . 3 - Determinação das pressões que atuam no fundo do silo (segundo a DIN1055-6)

$$
\mathrm{q}_{\mathrm{f}}=1,5 . \mathrm{q}_{\mathrm{c}}<8 * 17=136.000 \mathrm{~N} / \mathrm{m}^{2} \quad \mathrm{qf}_{\mathrm{f}}=1,5 * 88.641 \quad \mathrm{q}_{\mathrm{f}}=132.962 \mathrm{~N} / \mathrm{m}^{2}
$$


b) Determinação das Espessuras das Chapas

b.1 ) Tampa (Calculada segundo Gaylord \&Gaylord (1984), verificada de acordo com a NBR8800 (1986) ).

Tipo Cônica: inclinação de 1:2 $\left(26,6^{\circ}\right)$

suportada por vigas radiais apoiadas em dois anéis - um anel sobre o silo, diâmetro $11 \mathrm{~m}$, chamado anel de tração, o outro no topo do cone, diâmetro $1 \mathrm{~m}$, chamado de anel de compressão.

A ações são enumeradas para as combinações:

1. Ação do vento - já calculada no item a .1

2. Acidental (homem sobre a tampa), na posição mais desfavorável, meio do vão: $1.000 \mathrm{~N}$

3. Sobrecarga: $250 \mathrm{~N} / \mathrm{m}^{2}$

4. Permanente : peso próprio, estimado em $440 \mathrm{~N} / \mathrm{m}^{2}$

$\begin{array}{ccc}150 \mathrm{~N} / \mathrm{m}^{2} & 290 \mathrm{~N} / \mathrm{m}^{2} & 440 \mathrm{~N} / \mathrm{m}^{2} \\ \text { vigas } & \text { chapa de } 3,75 \mathrm{~mm} & \text { ação permanente }\end{array}$

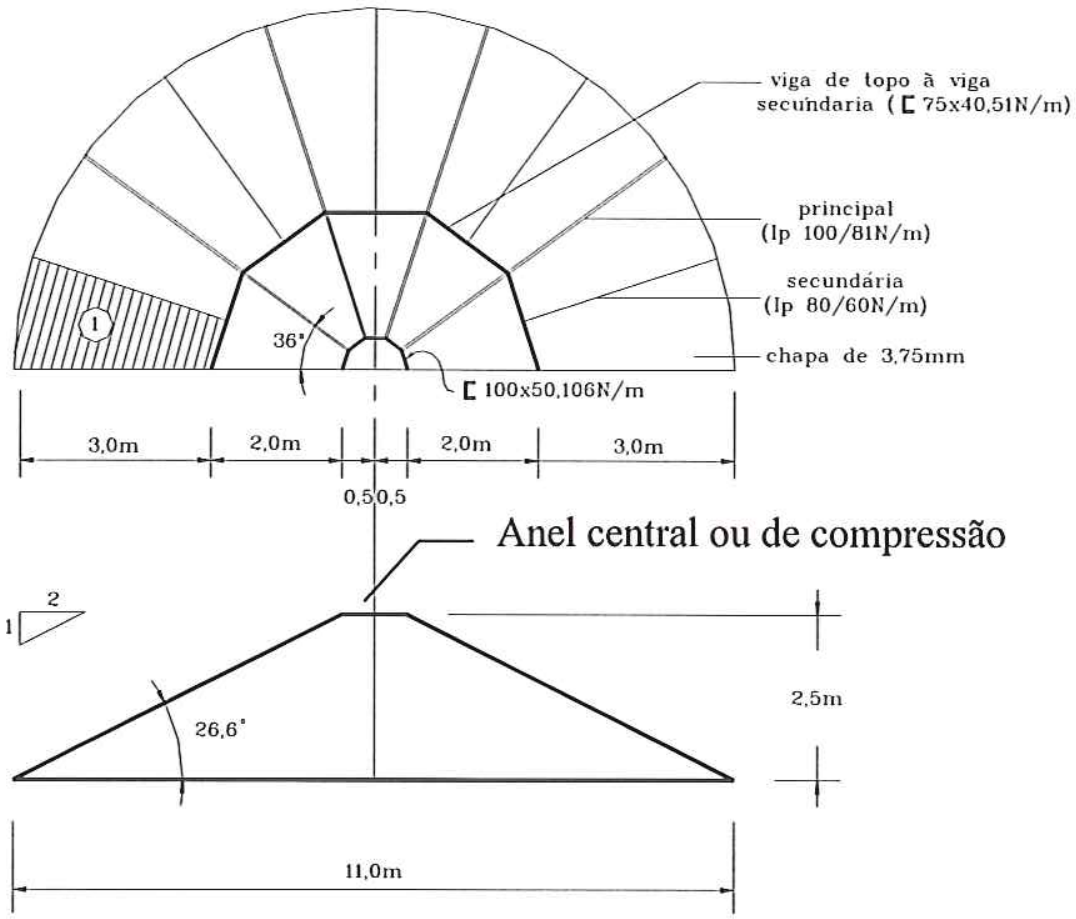

Figura 63 - Esquema da Cobertura Cônica 
No esquema da cobertura cônica são adotadas 10 vigas radiais principais ( $5 \mathrm{~m}$ ), 10 vigas radiais secundárias $(3 \mathrm{~m})$, de tal modo que no perímetro do topo do silo o espaçamento entre as vigas principais é $1,73 \mathrm{~m}$.

As vigas principais são consideradas apoiadas no anel central (anel de compressão).

O esquema de solicitações básicas sendo definido como:

\begin{tabular}{|c|c|}
\hline Número & descrição \\
\hline 1 & Permanente \\
\hline 2 & Sobrecarga \\
\hline 3 & Vento \\
\hline 4 & Acidental (concentrada) \\
\hline
\end{tabular}

Combinação de Ações:

mais desfavorável ( sem o efeito de sucção do vento)

$\mathrm{C}_{1}=1,3 \times 440+15 \times 250=947 \mathrm{~N} / \mathrm{m}^{2}$

considerando a sucção do vento, em seu valor mais desfavorável (faixa 4 da figura 75):

$\mathrm{C}_{2}=1,0 \times 440+1,4 \mathrm{x}(1128)+250=-890 \mathrm{~N} / \mathrm{m}^{2}$

As placas são analisadas de acordo com Gaylord \& Gaylord (1984) ( tabela 6-6 , pág.195) (também no apêndice), considerando um estado limite de utilização definido no anexo C da NBR8800 (1986).

\section{Placa 1}

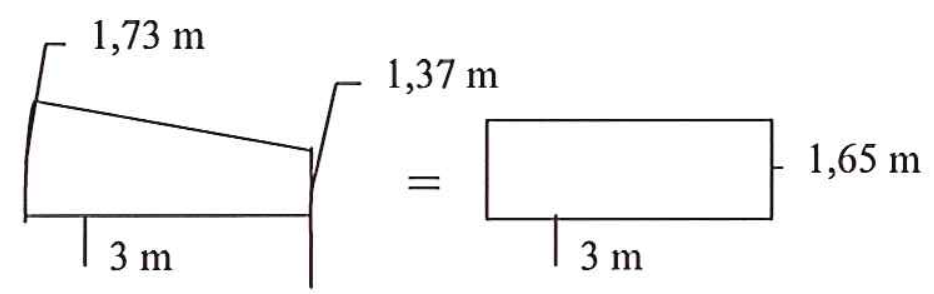

$\mathrm{b}=3,00 \mathrm{~m}, \mathrm{p}=250 \mathrm{~N} / \mathrm{m}^{2}, \mathrm{E}=205.000 \mathrm{Mpa}, \mathrm{t}=0,00375 \mathrm{~m}$

$$
\sqrt{\frac{p}{E}}\left(\frac{b}{t}\right)^{2}=\sqrt{\frac{250}{205 e 9}} \cdot 800^{2}=22,34 \quad \frac{b}{t}=\frac{3}{0,00375}=800
$$


Esse valor 22,34 implica, pela tabela $6-6$, na relação flecha $\delta$ no meio do maior vão (no caso $3 \mathrm{~m}$ ) para a espessura da placa $t: \quad \frac{\delta}{t}=2,74$ $\delta=0,00375 * 2,74 \cong 10 \mathrm{~mm}<17 \mathrm{~mm} \quad$ O.k., satisfaz a NBR8800 $\frac{3}{.00375} \sqrt{\frac{f}{205000}}=11 \Rightarrow \sqrt{\frac{f}{E}}=0,01375 \Rightarrow f \cong 38,76 \mathrm{Mpa}<250 \mathrm{Mpa}$ OK

$\underline{\text { Vigas Secundárias }}$

comprimento em projeção: $a=5,5-2,5 x \cos 18^{\circ}, a=3,12 \mathrm{~m}$.

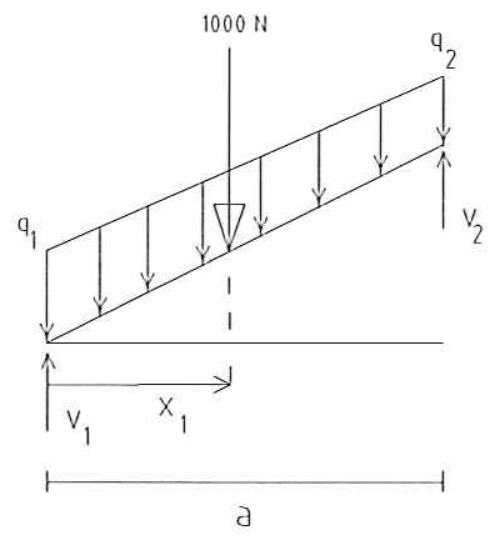

Figura 64 - Equilíbrio da Viga Secundária

$$
\begin{array}{ll}
q_{1}=690 \frac{11 \pi}{20} \cong 1192+100=1292 \mathrm{~N} / \mathrm{m} & V_{1}=\frac{3,122}{6}(2 * 1292+642)+\frac{1000}{2} \therefore V_{1} \cong 2180 \mathrm{~N} \\
q_{2}=690 \frac{5 \pi}{20} \cong 542+100=642 \mathrm{~N} / \mathrm{m} & V_{2}=\frac{3,122}{6}(1292+2 * 642)+\frac{1000}{2} \therefore V_{2} \cong 1840 \mathrm{~N}
\end{array}
$$

$\mathrm{O}$ momento atinge valor máximo em $\mathrm{x}_{1}$ :

$\frac{x_{1}}{a}=\frac{1292-\sqrt{\left(1292^{2}+1292 * 642+642^{2}\right) / 3}}{1292-642} \therefore \frac{x_{1}}{a}=0,472 \mathrm{~m}$

$M=\frac{3,122^{2}}{6} .0,492\left[2 * 1292+642-3 * 1292 * 0,492+(1292-642) 0,472^{2}\right]$

$M=1541+\underbrace{500 * \frac{3,122}{0,476}} \cong 2285 \mathrm{~N} . \mathrm{m}$

$\downarrow$ Referente à força de $1000 \mathrm{~N}$

Referente a carga distribuída 
Perfil escolhido : I 80 ( Manual Brasileiro de Estruturas Metálicas (1986) Vol. II, pág. 28 ) (ver também no Anexo 5)

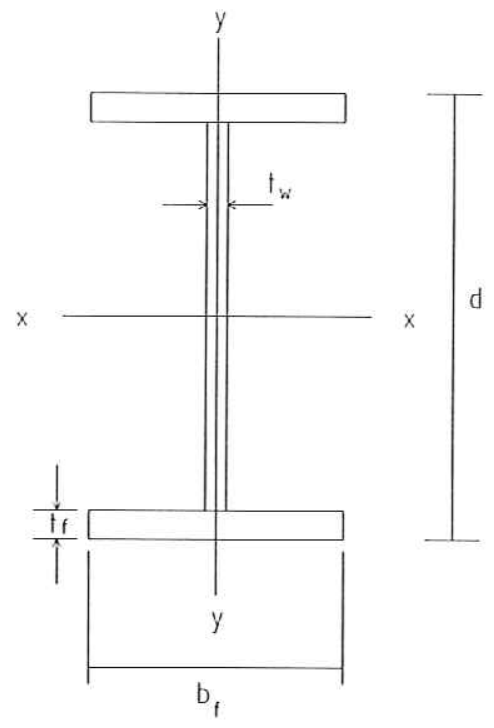

Figura 65 - Características do Perfil I adotado

\begin{tabular}{|c|c|c|c|c|}
\hline $\mathrm{d}=80 \mathrm{~mm}$ & $\mathrm{~b}_{\mathrm{f}}=46 \mathrm{~mm}$ & $\mathrm{t}_{\mathrm{W}}=3,8 \mathrm{~mm}$ & $\mathrm{t}_{\mathrm{f}}=5,2 \mathrm{~mm}$ & $\mathrm{p}=60 \mathrm{~N} / \mathrm{m}$ \\
\hline $\mathrm{A}=7,64 \mathrm{~cm}^{2}$ & $\mathrm{~W}_{\mathrm{X}}=20 \mathrm{~cm}^{3}$ & $\mathrm{I}_{\mathrm{X}}=80 \mathrm{~cm}^{4}$ & $\mathrm{r}_{\mathrm{y}}=1,05 \mathrm{~cm}$ & $\mathrm{I}_{\mathrm{t}}=0,70 \mathrm{~cm}^{4}$ \\
\hline
\end{tabular}

Classificação da Viga

$$
\frac{d}{t_{w}}=\frac{80}{3,8}=21<\lambda_{r}=5,6 \sqrt{\frac{E}{f_{y}}}=160 \quad \text { viga não-esbelta }
$$

Flambagem Local da Mesa - FLM

$$
\lambda=\frac{b}{t}=\frac{b_{f}}{2 t_{f}}=\frac{46}{2 \cdot 5,2}=4,42<\lambda_{p}=0,38 \sqrt{\frac{E}{f_{y}}} \cong 11
$$

Como $\lambda<\lambda_{\mathrm{p}}$, então $\mathrm{M}_{\mathrm{n}}=\mathrm{M}_{\mathrm{pl}}$

Flambagem Local da Alma - FLA

$$
\lambda=\frac{d}{t_{w}}=\frac{80}{3,8}=21 \quad \lambda_{p}=3,5 \sqrt{\frac{E}{f_{y}}} \cong 100
$$

Como $\lambda<\lambda_{\mathrm{p}}$

$M=M_{p l}$ 
$\underline{\text { Vigas de Topo às Vigas Secundárias }}$

Vão $=2 * 3 * \operatorname{sen} 18^{\circ}=1,85 \mathrm{~m}$

Peso da viga de topo

$$
\mathrm{V}_{2}=1840 \mathrm{~N}
$$

$47 \mathrm{~N}$

$1887 \mathrm{~N}$

Características do perfil adotado:

$$
\left[75 \times 40, P=51 \mathrm{~N} / \mathrm{m}, \mathrm{t}=4,76 \mathrm{~mm}, \mathrm{~W}_{\mathrm{x}}=21,1 \mathrm{~cm}^{3}, \mathrm{r}_{\mathrm{y}}=1,20 \mathrm{~cm}\right.
$$

\section{$\underline{\text { Vigas Principais }}$}

Inicialmente, é adotado um peso de $150 \mathrm{~N} / \mathrm{m}$ para a viga principal.

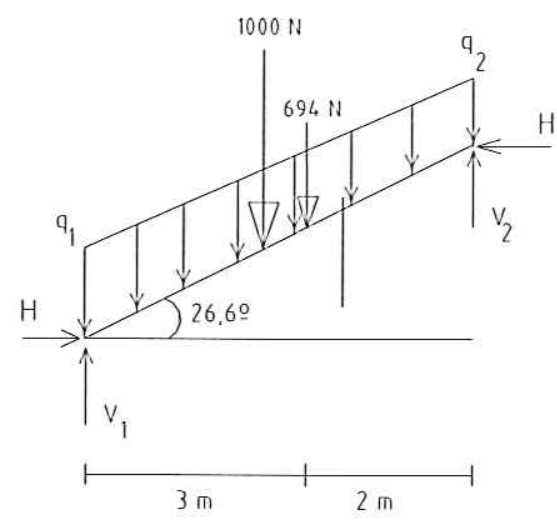

Figura 66 - Equilíbrio da Viga Principal

$$
\begin{aligned}
& q_{1}=1192+150=1342 \mathrm{~N} / m \quad q_{2}=690 \frac{2 \pi}{150}+150 \Rightarrow q_{2}=584 \mathrm{~N} / \mathrm{m} \\
& V_{1}=\frac{5}{2}(1342+584)+500+278 \Rightarrow V_{1}=5037 \mathrm{~N} \\
& V_{1}=\frac{5}{2}(1342+584)+500+416 \Rightarrow V_{1} \cong 5176 \mathrm{~N} \\
& H \operatorname{tg} \phi=\frac{5}{6}(1342+2 * 584)+500+416 \Rightarrow H \cong 6007 \mathrm{~N} \\
& \quad \text { O momento a } 3 \mathrm{~m}, \mathrm{x}_{1} / \mathrm{a}=3 / 5=0,6: \\
& M=2958 \mathrm{~N} \cdot \mathrm{m}
\end{aligned}
$$


Perfil Escolhido: Ip 100

\begin{tabular}{|c|c|c|c|c|}
\hline $\mathrm{d}=100 \mathrm{~mm}$ & $\mathrm{~b}_{\mathrm{f}}=55 \mathrm{~mm}$ & $\mathrm{t}_{\mathrm{W}}=4,1 \mathrm{~mm}$ & $\mathrm{t}_{\mathrm{f}}=5,7 \mathrm{~mm}$ & $\mathrm{p}=81 \mathrm{~N} / \mathrm{m}$ \\
\hline $\mathrm{A}=10,3 \mathrm{~cm}^{2}$ & $\mathrm{~W}_{\mathrm{X}}=34,3 \mathrm{~cm}^{3}$ & $\mathrm{I}_{\mathrm{X}}=171 \mathrm{~cm}^{4}$ & $\mathrm{r}_{\mathrm{y}}=1,24 \mathrm{~cm}$ & $\mathrm{I}_{\mathrm{t}}=1,21 \mathrm{~cm}^{4}$ \\
\hline
\end{tabular}

$\frac{d}{t_{w}}=\frac{100}{4,1}=24,4<\lambda_{r}=5,6 \sqrt{\frac{E}{f_{y}}}=160 \quad$ viga não-esbelta

Flambagem Lateral da Mesa - FLM

$$
\lambda=\frac{b}{t}=\frac{b_{f}}{2 t_{f}}=\frac{55}{2 * 5,7}=4,82<\lambda_{p}=0,38 \sqrt{\frac{E}{f_{y}}} \cong 11
$$

Como $\lambda<\lambda_{\mathrm{p}}$, então $\mathrm{M}_{\mathrm{n}}=\mathrm{M}_{\mathrm{pl}}$

Flambagem Local da Alma - FLA

$$
\lambda=\frac{d}{t_{w}}=\frac{100}{4,1}=24,4 \quad \lambda_{p}=3,5 \sqrt{\frac{E}{f_{y}}} \cong 100
$$

Como $\lambda<\lambda_{\mathrm{p}}$

$M=M_{p l}$

\section{Verificação Global das Vigas Principais}

Flambagem Lateral com Torção - FLT

$$
\begin{aligned}
& \lambda=\frac{l_{b}}{r_{y}}=\frac{312,2}{1,24} \cong 252 \lambda_{p}=1,75 \sqrt{\frac{E}{f_{y}}} \cong 50 \Rightarrow \lambda>\lambda_{p} \\
& \lambda_{r}=\frac{0,707 \beta_{1} C_{b}}{M_{r}} \sqrt{1+\sqrt{1+\frac{4 \beta_{2}}{C_{b}^{2} \beta_{1}^{2}} M_{r}^{2}}} \cong 315 \\
& \beta_{1}=\pi \sqrt{G E} \sqrt{I_{1} A} \cong 1420.10^{3} N . m \\
& \beta_{2}=6,145 \frac{A\left(d-t_{f}\right)^{2}}{I_{t}} \cong 4856 \quad C_{b}=1,0 \\
& M_{r}=W_{x}\left(f_{y}-f_{r}\right)=34,3(250-115) \therefore M_{r}=4630 \mathrm{~N} . \mathrm{m}
\end{aligned}
$$




$$
\begin{aligned}
& \lambda_{r} \cong 315>\lambda \quad \lambda_{p}=50<\underset{252}{\lambda_{2}<315=\lambda_{r}} \\
& M_{n}=M_{p l}-\left(M_{p l}-M_{r}\right) \frac{\lambda-\lambda_{p}}{\lambda_{r}-\lambda_{p}} \quad M_{p l}=34,3 * 250=8575 \mathrm{~N} . m \quad M_{p l}=M_{y} \\
& M_{n} \cong 5568 N . m . \\
& \phi_{b} M_{n}=0,9.5568=5011 N . m \quad M_{d} \cong 5011 N . m \\
& \delta\left(x_{1}=3,0 m\right)=\frac{864 * 3}{24 E 171.10^{-8}}\left(5^{3}-2 * 5 * 3^{2}+3^{3}\right) \\
& \delta=0,0191 m \cong 19 m m \Rightarrow \frac{19}{4470} \cong \frac{1}{235}
\end{aligned}
$$

\section{Anel de Compressão}

$\mathrm{O}$ anel de compressão está sujeito às solicitações de 10 vigas principais igualmente distribuídas no perímetro. A figura 48 ilustra o esquema de forças atuantes no anel e a força de compressão a que a seção está submetida:

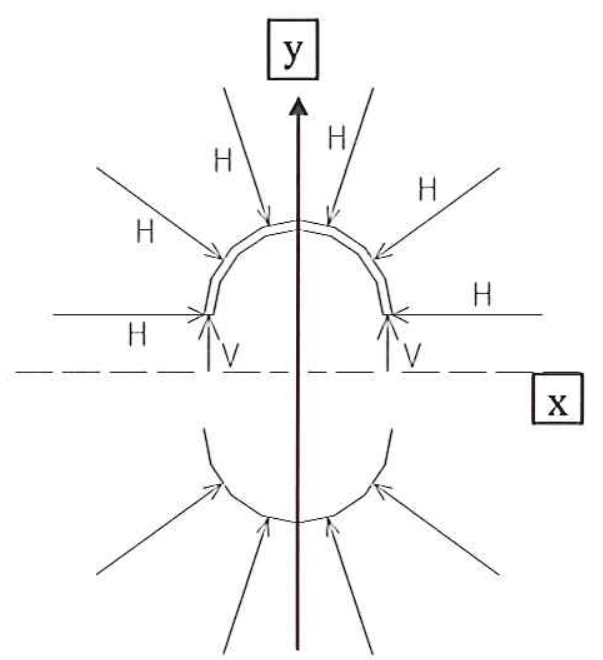

Figura 67 - Equilíbrio do Anel Central

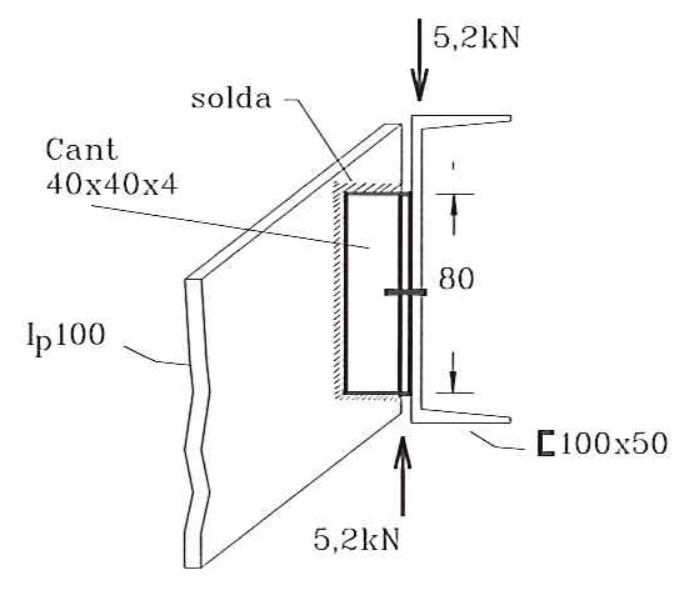

Figura 68 - Esquema do Anel Central 


\begin{tabular}{|l|l|}
\hline O.K. (simetria) & $2 V-2 H \operatorname{sen} 36^{\circ}-2 H \operatorname{sen} 72^{\circ}=0$ \\
& $V=H\left(\operatorname{sen} 36^{\circ}+\operatorname{sen} 72^{\circ}\right)$ \\
& $V \cong 9244 N$ \\
\hline
\end{tabular}

Admitindo um perfil IP-100 - de acordo com a Tabela 3 do Anexo 1 - que tem área transversal $A=13,5 \mathrm{~cm}^{2}$ :

$$
\frac{V}{A}=\frac{9244 N}{0,00135 m^{2}} \cong 6,85 M P a \quad \text { O.K. }
$$




\section{Anel Tracionado}

As forças radiais que atuam no anel tracionado são as mesmas que atuam no anel central. Logo, as forças que atuam no anel tracionado têm a mesma resultante $\mathbf{V}$ (figura 82). $\mathrm{O}$ anel tracionado é admitido com a seção indicada na figura 84 :

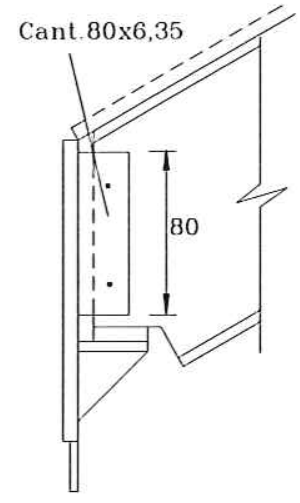

a) Ligação no anel

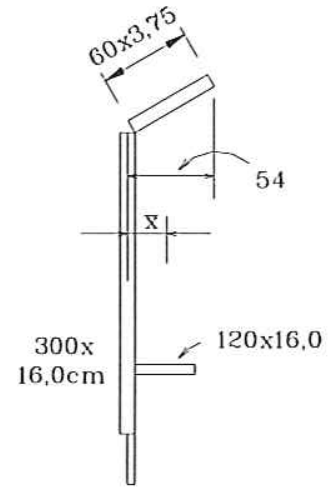

b) Detalhe do anel

Figura 69 - Seção transversal do anel tracionado.

A área da seção do anel tracionado é $\mathrm{A}=69,45 \mathrm{~cm}^{2}$, que, para $\mathrm{V}=9244 \mathrm{~N}$ resulta numa tensão igual a $0,133 \mathrm{kN} / \mathrm{cm}^{2}$

$\underline{\text { Cálculo dos Parafusos de ligação da: }}$

- Viga principal ao anel central (figura 83):

2 parafusos ASTM A307 1/2" (figura 85)

solicitação: (esforço cortante $=5,2 \mathrm{KN}) \quad$ resistência: $13,25 \mathrm{KN}$

- Viga secundária à viga de topo (o detalhe para a chapa de ligação é semelhante ao da figura 85 , a menos da altura de $80 \mathrm{~mm}$, que deve ser $60 \mathrm{~mm}$, para se adequar ao IP 80):

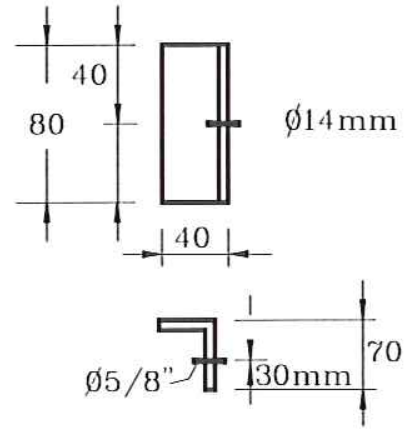

Figura 70 - Detalhe da chapa para a viga principal

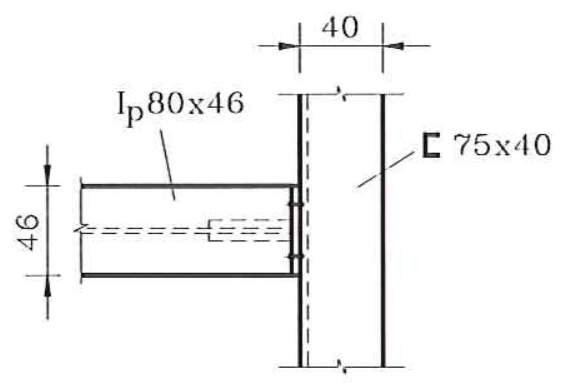

Figura 71 - Detalhe da ligação da viga de topo à viga secundária 
b.2 ) Determinação das espessuras das chapas da parede cilíndrica

b.2.1 ) Combinação das Ações.

$\mathrm{C}_{1}=1,0$ p.p. $+1,4 \cdot$ vento

peso-próprio (р.p.) : (peso da tampa, por metro de perímetro ) + peso das chapas por metro linear do perímetro ao longo da altura.

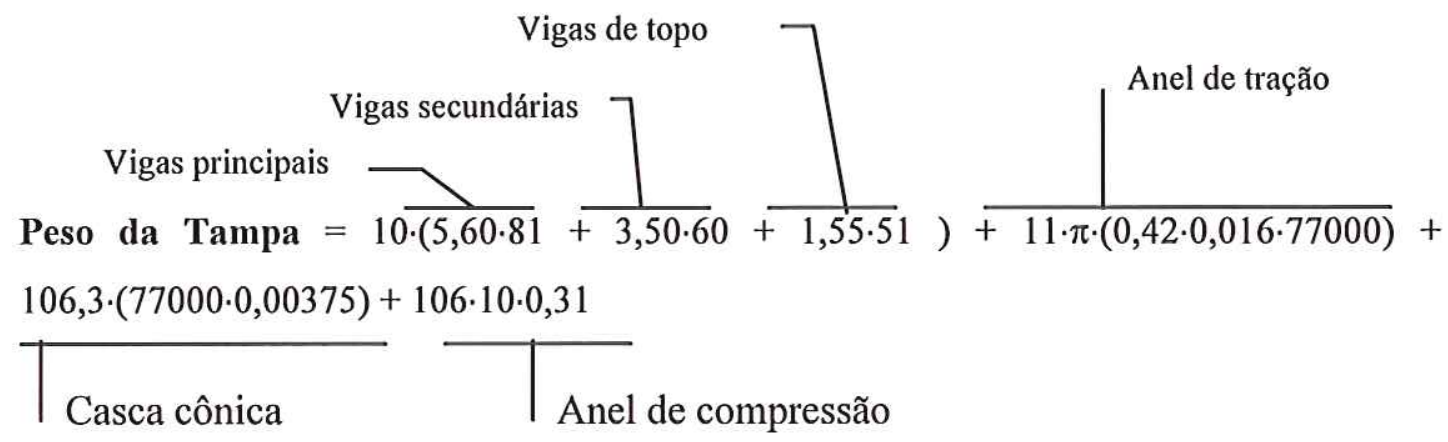

\begin{tabular}{|c|c|}
\hline Peso da Tampa $=7.427+17.881+30.684+329$ & Peso da Tampa $=56.321 \mathrm{~N}$ \\
\hline Perímetro $=11 \mathrm{~m} * \mathrm{p}=34,56 \mathrm{~m}$ & $\frac{\text { Peso.da.tampa }}{\text { perimetro }} \cong 1630 \mathrm{~N} / \mathrm{m}$ \\
\hline
\end{tabular}




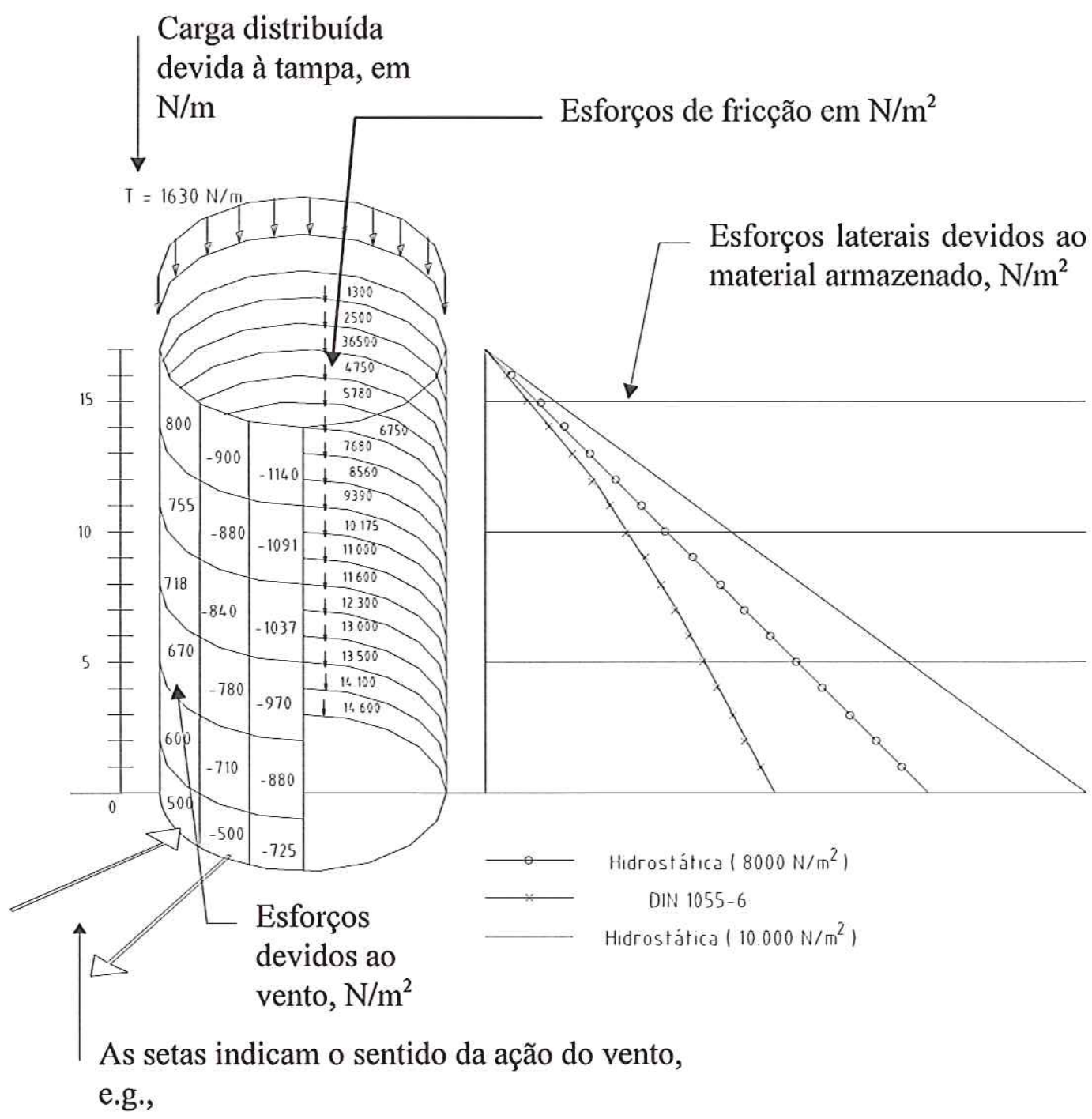

Figura 72 - Esquema de aplicação das forças no costado

O cálculo numérico, via elementos finitos, é determinado utilizando-se o programa ANSYSC. Os resultados numéricos estão indicados em figuras que indicam as áreas de mesma tensão meridional, nos casos de solicitação para o vento e para o material ensilado. Ao final deste capítulo, estão anexados os resultados de deslocamentos e tensões meridionais e tangenciais, ao longo de uma linha meridional, para o caso do material ensilado, devido à axissimetria do modelo. 
b.2.2 ) Esquema das Malhas e da Rede de Elementos Finitos para o Silo Exemplo

Considerando-se a ação do vento e a geometria axissimétrica da estrutura, foi adotado um modelo simétrico, formado por metade da casca cilíndrica do silo exemplo.

Além disso, esse modelo foi dividido em 10 áreas de interesse para a análise da estrutura, e foi fixada uma origem global. A figura 88 ilustra o modelo, cuja base foi considerada com restrições aos deslocamentos e aos giros nas direções x, y e z. As figuras posteriores e mostram a distribuição dos elementos e dos nós no modelo.

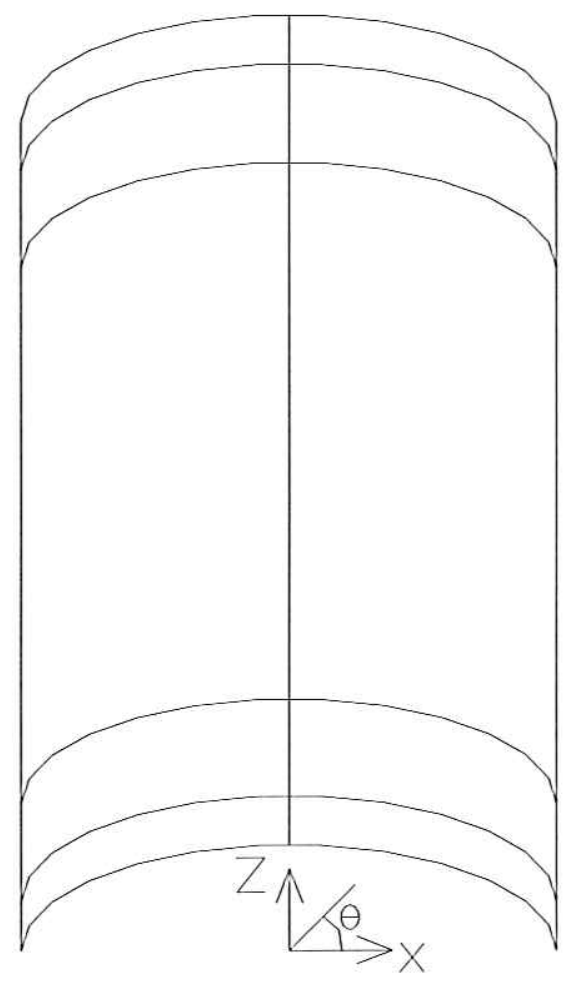

\begin{tabular}{|c|c|}
\hline А́геа 10 & А́геа 1 \\
\hline А́геа 9 & А́геа 2 \\
\hline Área 8 & Área 3 \\
\hline Área 7 & Área 4 \\
\hline Агеа 6 & Area 5 \\
\hline
\end{tabular}

Figura 73- Definição das Áreas do Silo-exemplo. 


\begin{tabular}{|l|l|l|l|l|l|l|l|l|}
\hline 882 & & 880 & & & & $\leftarrow$ & & 874 \\
\hline 873 & & & 870 & & & & & 865 \\
\hline 864 & & & & 860 & & & & 856 \\
\hline 855 & & & & & 850 & & & 847 \\
\hline 846 & 845 & & & & & 840 & & 838 \\
\hline 837 & & 835 & & & & & 830 & 829 \\
\hline 828 & & & 825 & & & & & 820 \\
\hline 819 & & & & 815 & & & & 811 \\
\hline 810 & & & & & 805 & & & 802 \\
\hline 801 & 800 & & & & & 795 & & 793 \\
\hline
\end{tabular}

\begin{tabular}{|l|l|l|l|l|l|l|l|l|}
\hline 531 & 530 & & & $\leftarrow$ & & 525 & & 523 \\
\hline 540 & & & & & 535 & & & 532 \\
\hline 549 & & & & 545 & & & & 541 \\
\hline 558 & & & 555 & & & & & 550 \\
\hline 567 & & 565 & & & & & 560 & 559 \\
\hline 576 & 575 & & & & & 570 & & 568 \\
\hline 585 & & & & & 580 & & & 577 \\
\hline 594 & & & & 590 & & & & 586 \\
\hline 603 & & & 600 & & & & & 595 \\
\hline 612 & & 610 & & & & & & 604 \\
\hline
\end{tabular}

\begin{tabular}{|l|l|l|l|l|l|l|l|l|}
\hline 324 & & & & 320 & & $\leftarrow$ & & 316 \\
\hline 315 & & & & & 310 & & & 307 \\
\hline 306 & 305 & & & & & 300 & & 298 \\
\hline 297 & & 295 & & & & & 290 & 289 \\
\hline
\end{tabular}

\begin{tabular}{|l|l|l|l|l|l|l|l|l|}
\hline 189 & & & & 185 & & $\leftarrow$ & & 181 \\
\hline 198 & & & 195 & & & & & 190 \\
\hline 207 & & 205 & & & & & 200 & 199 \\
\hline 216 & 215 & & & & & 210 & & 208 \\
\hline
\end{tabular}

\begin{tabular}{|l|l|l|l|l|l|l|l|l|}
\hline 522 & & 520 & & & $\leftarrow$ & & 515 & 514 \\
\hline 513 & & & 510 & & & & & 505 \\
\hline 504 & & & & 500 & & & & 496 \\
\hline 405 & & & & & 490 & & & 487 \\
\hline 485 & 485 & & & & & 480 & & 478 \\
\hline 477 & & 475 & & & & & 470 & 469 \\
\hline 468 & & & 465 & & & & & 460 \\
\hline 459 & & & & 455 & & & & 451 \\
\hline 450 & & & & & 445 & & & 442 \\
\hline 441 & 440 & & & & & 435 & & 433 \\
\hline 432 & & 430 & & & & & 425 & 424 \\
\hline
\end{tabular}

\begin{tabular}{|l|l|l|l|l|l|l|l|l|}
\hline 333 & & & 330 & & $\leftarrow$ & & & 325 \\
\hline 342 & & 340 & & & & & 335 & 334 \\
\hline 351 & 350 & & & & & 345 & & 343 \\
\hline 360 & & & & & 355 & & & 352 \\
\hline 369 & & & & 365 & & & & 361 \\
\hline 378 & & & 375 & & & & & 370 \\
\hline 387 & & 385 & & & & & 380 & 379 \\
\hline 396 & 395 & & & & & 390 & & 388 \\
\hline 405 & & & & & 400 & & & 397 \\
\hline 414 & & & & 410 & & & & 406 \\
\hline 423 & & & 420 & & & & & 415 \\
\hline
\end{tabular}

\begin{tabular}{|l|l|l|l|l|l|l|l|l|}
\hline 288 & & & 285 & & & $\leftarrow$ & & 280 \\
\hline 279 & & & & 275 & & & & 271 \\
\hline 270 & & & & & 265 & & & 262 \\
\hline 261 & 260 & & & & & 255 & & 253 \\
\hline
\end{tabular}

\begin{tabular}{|l|l|l|l|l|l|l|l|l|}
\hline 225 & & & $\leftarrow$ & & 220 & & & 217 \\
\hline 234 & & & & 230 & & & & 226 \\
\hline 243 & & & 240 & & & & & 235 \\
\hline 252 & & 250 & & & & & & 244 \\
\hline
\end{tabular}

\begin{tabular}{|l|l|l|l|l|l|l|l|l|}
\hline 792 & & 790 & & $\leftarrow$ & & & 785 & 784 \\
\hline 783 & & & 780 & & & & & 775 \\
\hline 774 & & & & 770 & & & & 766 \\
\hline 765 & & & & & 760 & & & 757 \\
\hline 756 & 755 & & & & & 750 & & 748 \\
\hline 747 & & 745 & & & & & 740 & 39 \\
\hline 738 & & & 735 & & & & & 730 \\
\hline 729 & & & & 725 & & & & 721 \\
\hline 720 & & & & & 715 & & & 712 \\
\hline 711 & 710 & & & & & 705 & & 703 \\
\hline
\end{tabular}

\begin{tabular}{|l|l|l|l|l|l|l|l|l|}
\hline 621 & 620 & & $\leftarrow$ & & & 615 & & 613 \\
\hline 630 & & & & & 625 & & & 622 \\
\hline 639 & & & & 635 & & & & 631 \\
\hline 648 & & & 645 & & & & & 640 \\
\hline 657 & & 655 & & & & & 650 & 649 \\
\hline 666 & 665 & & & & & 660 & & 658 \\
\hline 675 & & & & & 670 & & & 667 \\
\hline 684 & & & & 680 & & & & 676 \\
\hline 693 & & & 690 & & & & & 685 \\
\hline 702 & & 700 & & & & & 695 & 694 \\
\hline
\end{tabular}

Figura 74 - Mapa dos Elementos Finitos na Casca do Silo Exemplo. 

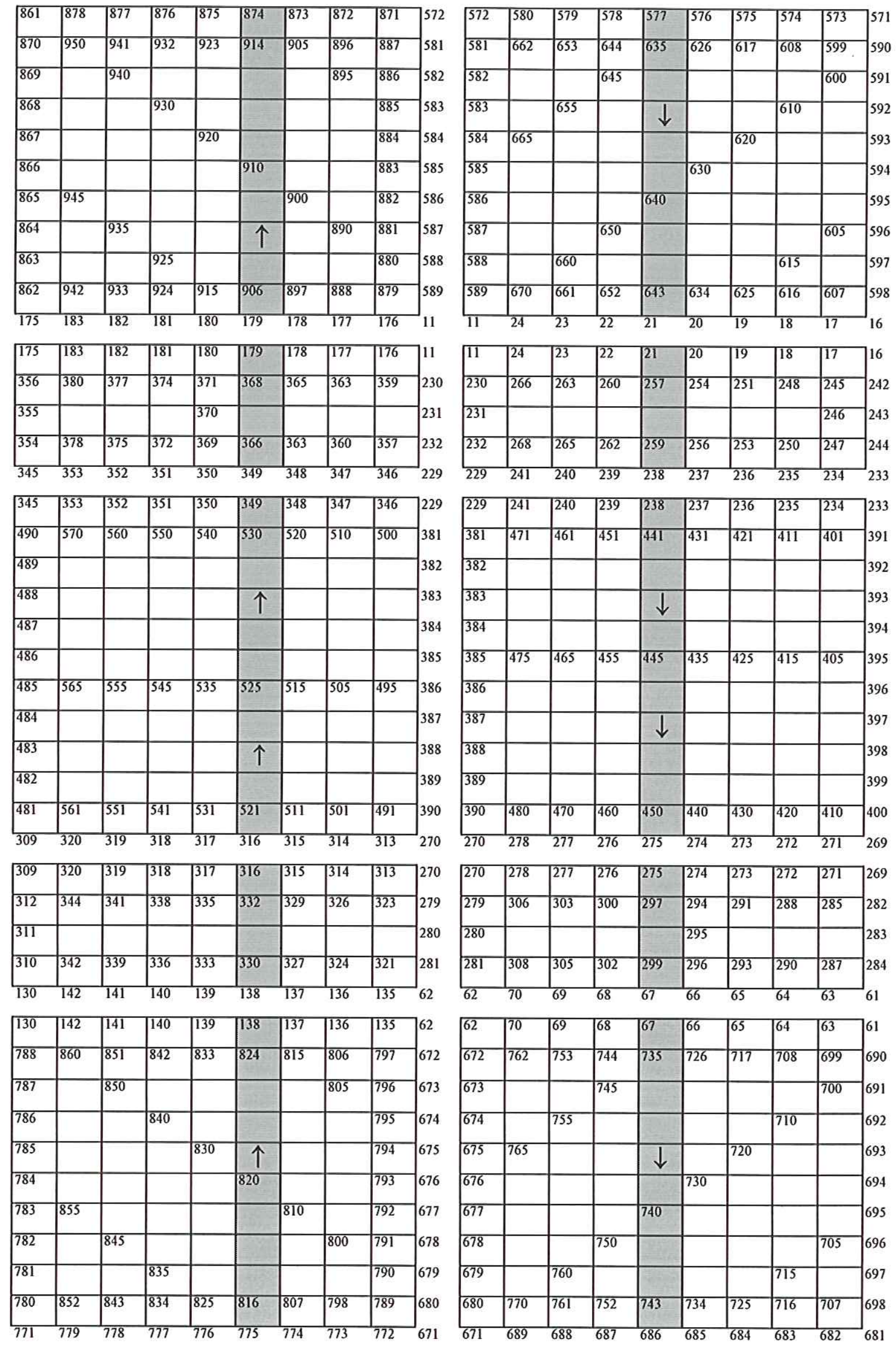

Figura 75 - Mapa dos Nós dos Elementos Finitos na Casca do Silo Exemplo 


\section{b.2.3) Verificações à Flambagem}

Para essa configuração de elementos finitos, foram ensaiados no ANSYS cinco variações de espessura ( em milímetros ) e escolhida a quinta variação :

\begin{tabular}{|c|c|c|c|c|c|c|c|}
\hline Faixas & $1^{\mathrm{a}}$ & $2^{\mathrm{a}}$ & Faixas & $3^{\mathrm{a}}$ & $4^{\mathrm{a}}$ & Faixas & $5^{\mathrm{a}}$ \\
\hline $17-16 \mathrm{~m}$ & 6,3 & 6,3 & $16-17 \mathrm{~m}$ & 6,3 & 4,75 & - & - \\
\hline $16-14 \mathrm{~m}$ & 6,3 & 6,3 & $14-16 \mathrm{~m}$ & 6,3 & 4,75 & $7-17$ & 4,75 \\
\hline $3-14 \mathrm{~m}$ & 6,3 & 4,75 & $3-14 \mathrm{~m}$ & 4,75 & 4,75 & $4-7 \mathrm{~m}$ & 6,3 \\
\hline & - & - & $2-3 \mathrm{~m}$ & 8,0 & 8,0 & $2-4 \mathrm{~m}$ & 8,0 \\
\hline $1-3 \mathrm{~m}$ & 9,5 & 8,0 & $1-2 \mathrm{~m}$ & 9,5 & 9,5 & $1-2 \mathrm{~m}$ & 9,5 \\
\hline $0-1 \mathrm{~m}$ & 12,5 & 9,5 & $0-1 \mathrm{~m}$ & 12,5 & 12,5 & $0-1 \mathrm{~m}$ & 16,0 \\
\hline \multicolumn{7}{|c|}{ TABELA 14 - Variações de espessura testadas para o silo-exemplo } \\
\hline
\end{tabular}

O critério para a avaliação das 5 variações testadas é o de flambagem. São verificadas a flambagem para o silo vazio e sob a ação do (verificação de flexão + compressão), para o silo cheio com milho (verificação de compressão + pressão interna). Além disso, uma verificação prévia, apenas para a expressão clássica de cilindro axialmente comprimido também foi efetuada (eq. 46). O resultado superestima o valor da carga crítica.

- Verificação de Flexão + compressão (solicitação do vento - silo vazio)

O peso-próprio da tampa foi computado para se obter $\sigma_{0}$, que é a tensão uniforme de compressão devida à força axial de projeto .

Dos resultados do ANSYS, é obtido o valor da tensão máxima de compressão meridional, $\sigma_{b}=9,73 \mathrm{MPa}$, sendo analisada as espessuras de chapa onde atua essa tensão ( $4,75 \mathrm{~mm}, 6,3 \mathrm{~mm}$ e $12,5 \mathrm{~mm}$, para as regiões em azul na figura ). Foi escolhida essa figura porque são as tensões máximas atuantes no elemento (na face interna da casca - $\mathrm{S}_{\mathrm{Z}}$ botton)

Para uma tensão de compressão $\sigma_{\mathrm{b}}=-7,9 \mathrm{MPa}$, os resultados são:

\begin{tabular}{|c|c|c|c|c|}
\hline $\begin{array}{c}\text { Espessuras } \\
\mathrm{m}\end{array}$ & $\begin{array}{c}\text { Tensão Crítica } \\
\text { (MPa) }\end{array}$ & Alfa & $\begin{array}{c}\text { Tensão Obtida } \\
(\mathrm{MPa})\end{array}$ & \\
\hline 0,00475 & 107 & 0,827 & 66,42 & $<107$ \\
\hline 0,00630 & 142 & 0,901 & 96,02 & $<142$ \\
\hline 0,00800 & 180 & 0,973 & & \\
\hline 0,00950 & 214 & 1,029 & & \\
\hline 0,01600 & 242 & 1,130 & 161 & 242 \\
\hline
\end{tabular}

TABELA 15 - Verificação de flambagem para flexão + compressão 
- Verificação de compressão + pressão interna

Para esta verificação precisa-se determinar a tensão de membrana de projeto, $\sigma_{\mathrm{Zd}}$, a pressão interna estática $\mathrm{p}_{\mathrm{c}}$ (de Janssen) correspondente a $\sigma_{\mathrm{Zd}}$, e dispor da tensão de escoamento do aço, do raio e da espessura na seção. Pondo em forma de um roteiro:

1. Dado $\sigma_{\mathrm{zd}}$

2. Calcula-se a tensão tangencial, $\sigma_{\theta}=-p_{c} \frac{R}{e}$

3. Calcula-se a tensão efetiva (eq.51) $\sigma_{e f, d}=\sqrt{\sigma_{z d}^{2}+\sigma_{a l}^{2}-\sigma_{a l} \sigma_{z d}}$

4. Acha-se (eq. 58) o parâmetro $\beta=\frac{\sigma_{z d}}{\sigma_{e f, d}}$

5. Pela eq. 57, calcula-se $\rho=\frac{\sigma_{a l}}{E} \sqrt{\frac{R}{e}}$

6. Determina-se $\alpha_{0}$ (eq. 49 ou eq. 50 , dependendo do valor de R/e)

7. Calcula-se $\alpha_{p}=\alpha_{0}+\left(1-\alpha_{0}\right) \frac{\rho}{\rho+0,007}$

8. Acha-se $\lambda_{p}=\sqrt{\frac{\beta f_{y}}{\alpha_{p} \sigma_{c r}}}$

9. Finalmente, obtém-se $\sigma_{\text {ef,u }}$ com uma das equações 53 ou 54 .

10. Compara-se $\sigma_{\text {ef,d }}<\sigma_{\text {ef,u }}$.

\begin{tabular}{|c|c|c|c|c|c|c|c|c|c|c|}
\hline Espessuras & pc & $(\sigma \mathrm{Z})$ & $\sigma \theta$ & $\sigma e f, d$ & $\beta$ & $\rho$ & $\alpha 0$ & $\alpha p$ & $\lambda p$ & $\sigma e f, u$ \\
\hline \multicolumn{11}{|c|}{ CASO 5} \\
\hline 4,75 & 0.036 & 22 & -41.68 & 56.02 & 0.393 & 0.0069 & 0.2048 & 0.600106 & 1.236 & 78.66 \\
\hline 6,30 & 0.047 & 22 & -41.03 & 55.41 & 0.397 & 0.0059 & 0.2356 & 0.585639 & 1.092 & 122.61 \\
\hline 8,00 & 0.050 & 40 & -34.38 & 64.47 & 0.62 & 0.0044 & 0.2650 & 0.548583 & 1.252 & 73.26 \\
\hline 9,50 & 0.051 & 45 & -29.53 & 65 & 0.692 & 0.0035 & 0.2884 & 0.524068 & 1.242 & 76.74 \\
\hline 16,00 & 0.053 & 59 & -18.22 & 69.91 & 0.844 & 0.0016 & 0.3722 & 0.491802 & 1.234 & 79.38 \\
\hline
\end{tabular}

As figuras e as tabelas de onde são obtidos os valores para o cálculo das tabelas 16 e 17 são fornecidos primeiro para a verificação de flexão + compressão, e depois para o caso de compressão + pressão interna: 


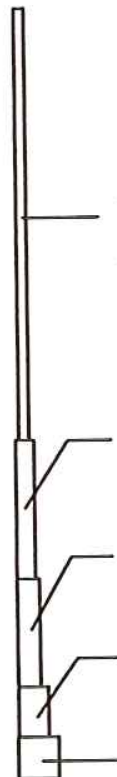

$7,0-17 \mathrm{~m}$ $4,75 \mathrm{~mm}$

$4,0-7,0 \mathrm{~m}$ $6,3 \mathrm{~mm}$

$2,0-4,0 \mathrm{~m}$

$8,0 \mathrm{~mm}$

$1,0-2,0 \mathrm{~m}$ $9,5 \mathrm{~mm}$ $0-1,0 \mathrm{~m}$ $16,0 \mathrm{~mm}$

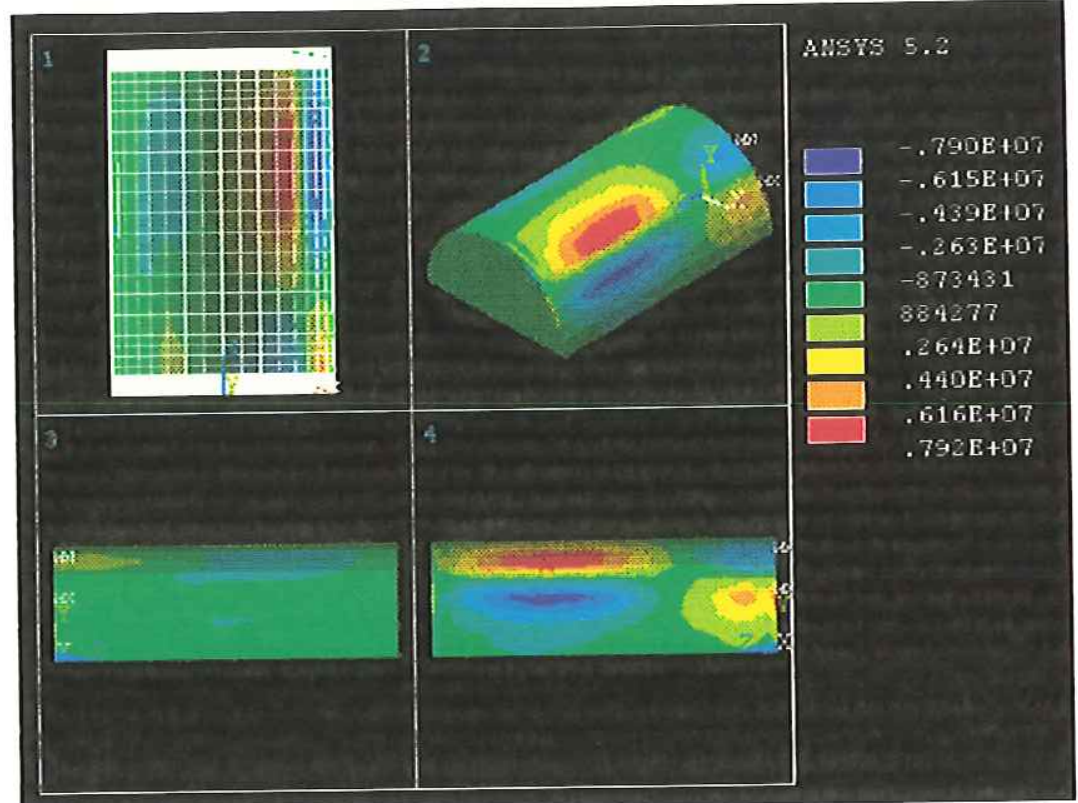

Figura 76 - Tensões meridionais (vento): face interna da casca
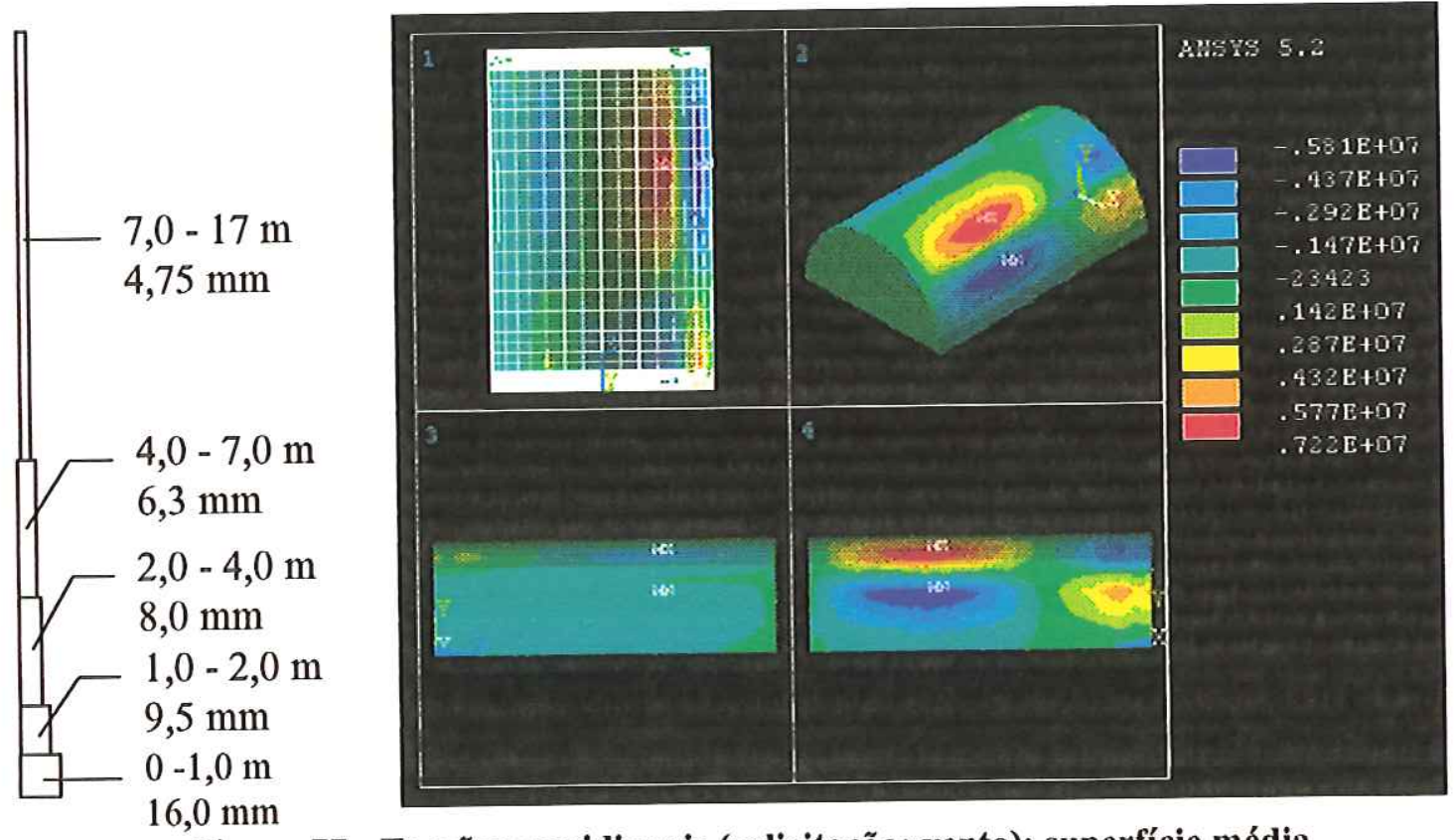

Figura 77 - Tensões meridionais (solicitação: vento): superfície média 


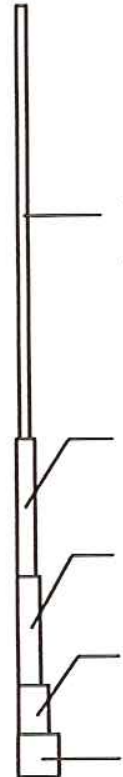

$7,0-17 \mathrm{~m}$ $4,75 \mathrm{~mm}$

$4,0-7,0 \mathrm{~m}$ $6,3 \mathrm{~mm}$

$2,0-4,0 \mathrm{~m}$ $8,0 \mathrm{~mm}$

$1,0-2,0 \mathrm{~m}$ $9,5 \mathrm{~mm}$ $0-1,0 \mathrm{~m}$ $16,0 \mathrm{~mm}$

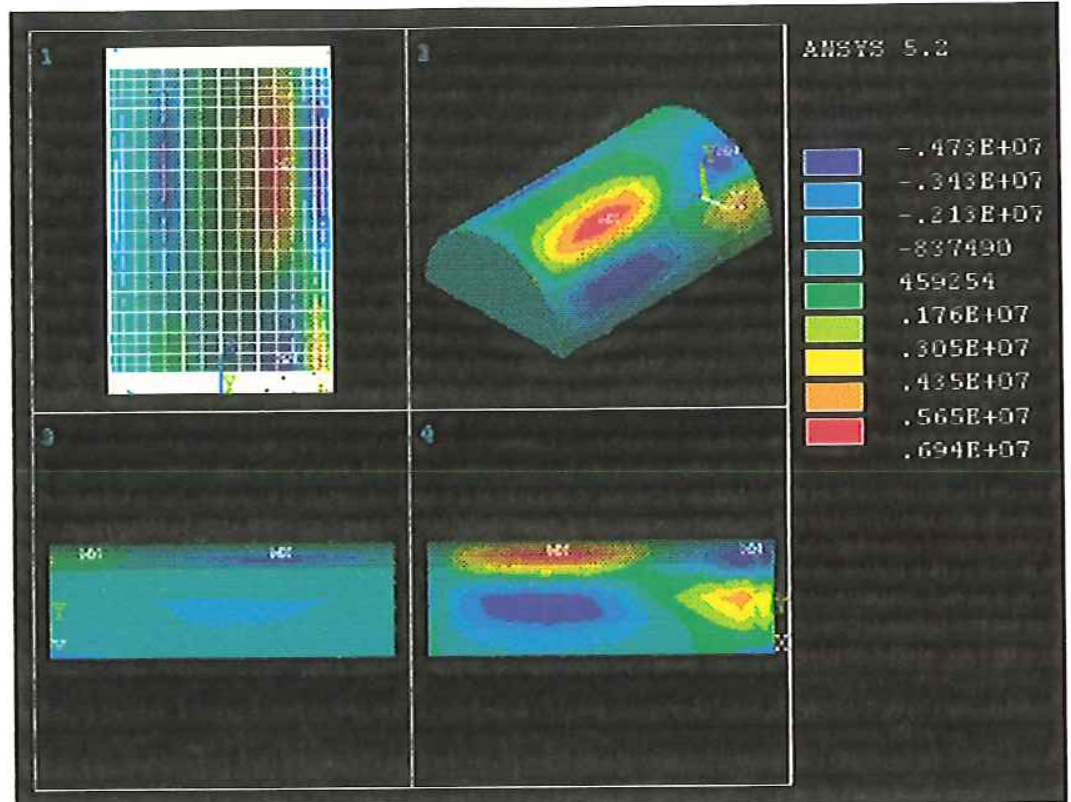

Figura 78 - Tensões meridionais (solicitação: vento): face externa da casca

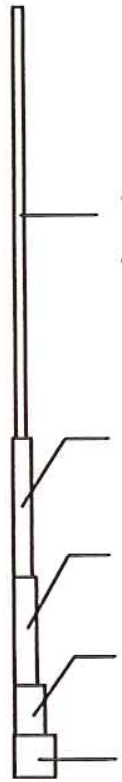

$7,0-17 \mathrm{~m}$ $4,75 \mathrm{~mm}$

$4,0-7,0 \mathrm{~m}$ $6,3 \mathrm{~mm}$ $2,0-4,0 \mathrm{~m}$ $8,0 \mathrm{~mm}$ $1,0-2,0 \mathrm{~m}$ $9,5 \mathrm{~mm}$ $0-1,0 \mathrm{~m}$ $16,0 \mathrm{~mm}$

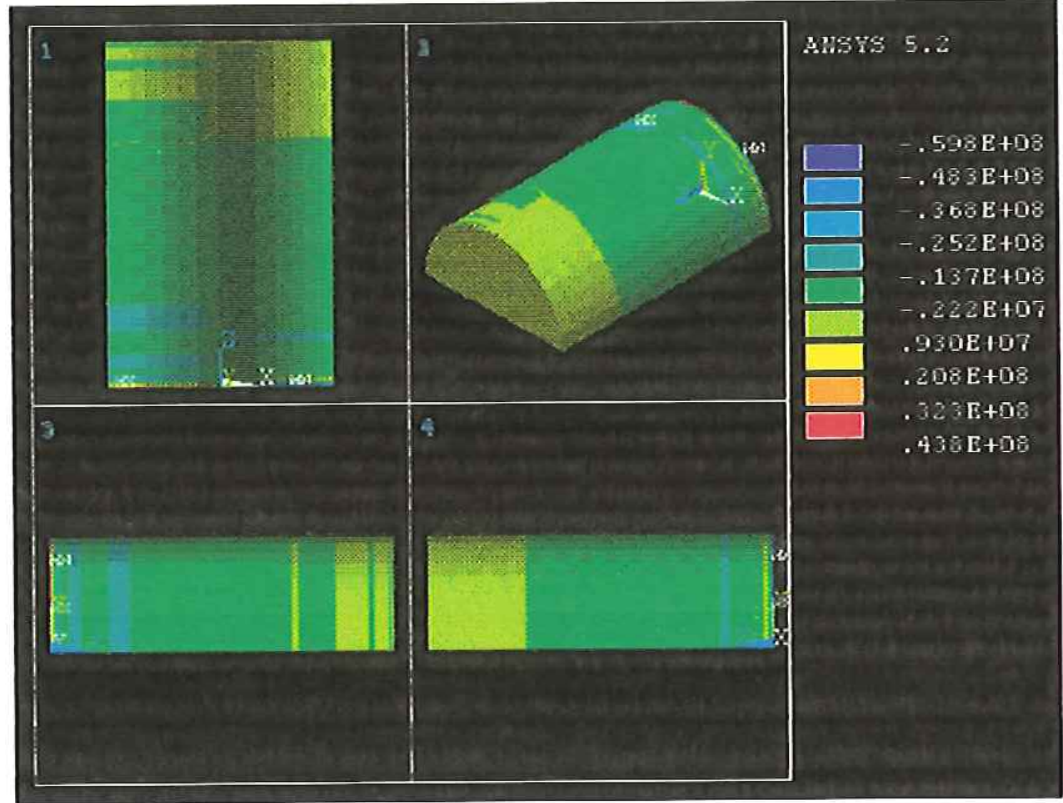
Figura 79 - Tensões meridionais (solicitação: grãos): face interna da casca 

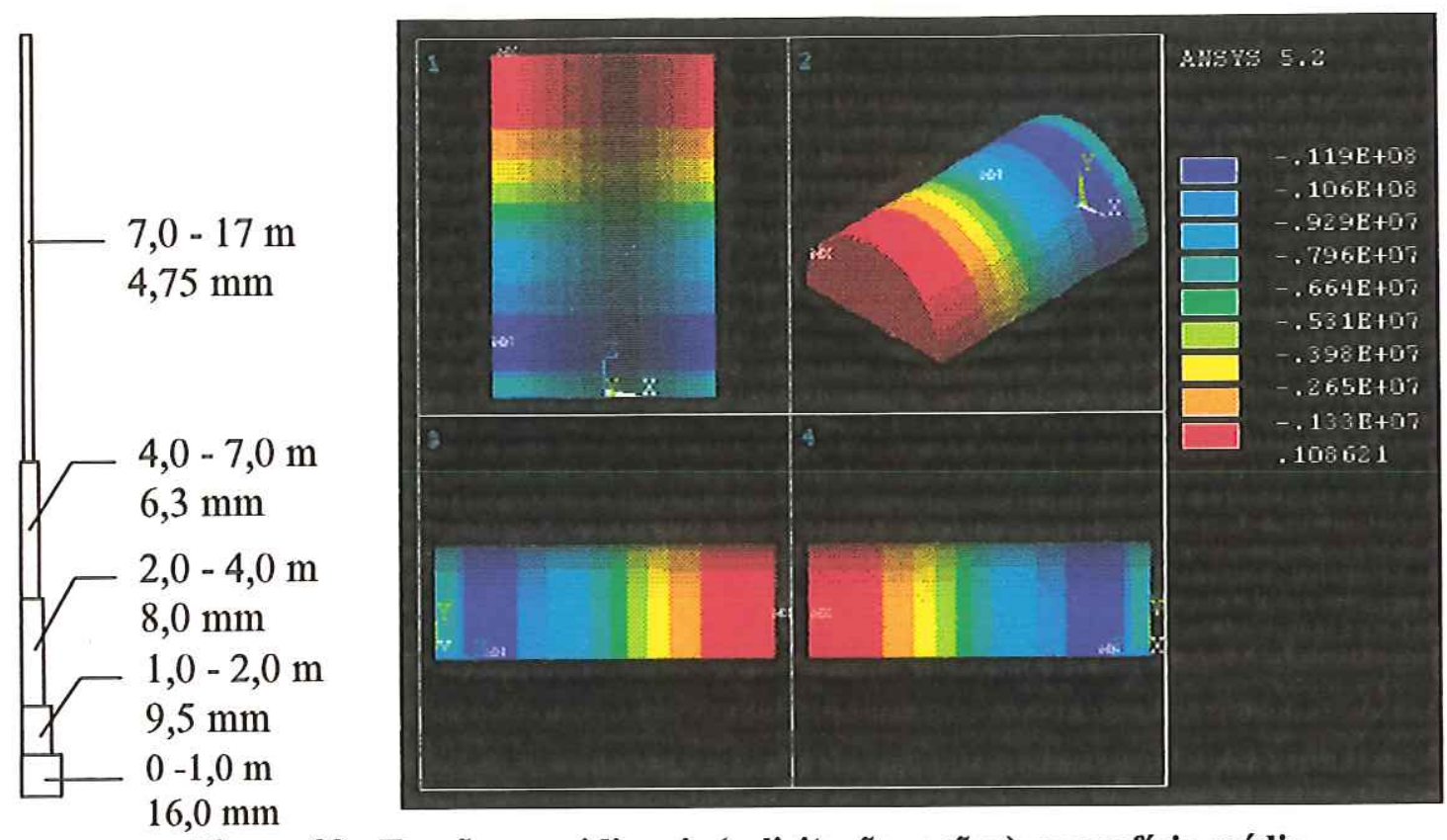

Figura 80 - Tensões meridionais (solicitação: grãos): superfície média

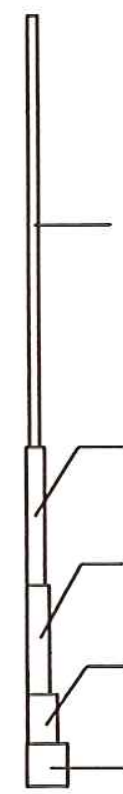

$7,0-17 \mathrm{~m}$ $4,75 \mathrm{~mm}$

$4,0-7,0 \mathrm{~m}$ $6,3 \mathrm{~mm}$ $2,0-4,0 \mathrm{~m}$ $8,0 \mathrm{~mm}$ $1,0-2,0 \mathrm{~m}$ $9,5 \mathrm{~mm}$ $0-1,0 \mathrm{~m}$ $16,0 \mathrm{~mm}$

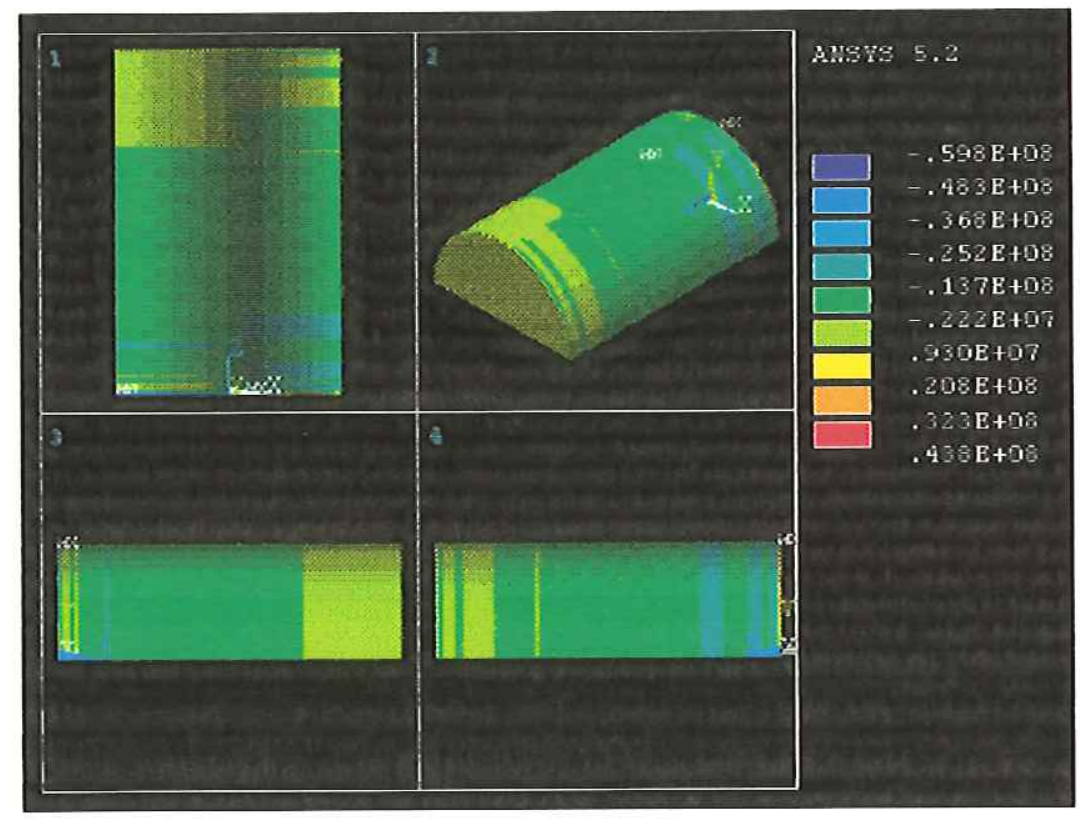

Figura 81 - Tensões meridionais (solicitação: grãos): face externa da casca 
Concluída a verifícação à flambagem do costado do silo de grãos, as espessuras das chapas do costado são:
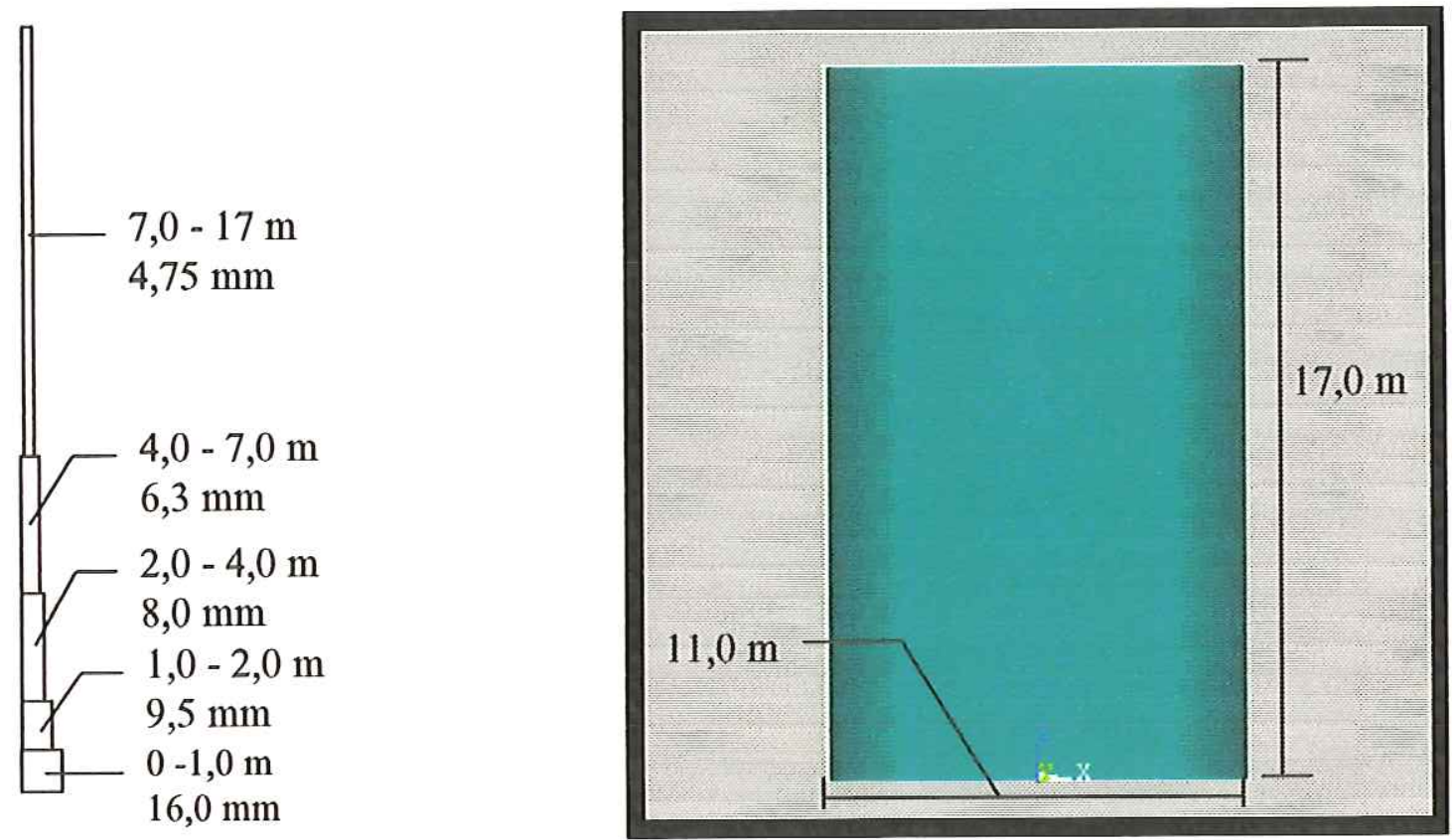

Figura 82 - Espessuras do Costado do Silo Exemplo. 


\section{Capítulo 6 - Conclusões}

O comportamento das estruturas em cascas, de geometria cilíndrica, teve enfoque à aplicação em silos e em reservatórios metálicos de paredes delgadas $(400<\mathrm{r} / \mathrm{e}<500)$. As características que diferenciam um silo de um reservatório foram apresentadas e o fenômeno de flambagem foi analisado para tipos determinados de solicitação da estrutura. As conclusões serão feitas com base nos modelos 1 e $5(\mathrm{H} / \mathrm{D}=1$, e H/D $=5$, respectivamente) da análise numérica, e esclarecidas segundo a teoria apresentada no capítulo 3. Serão comparados os resultados numéricos aos métodos de verificação da norma européia [ECCS (1988)].

Para as estruturas de silos e de reservatórios de relação altura/diâmetro 1 (modelo 1), a flambagem pode ocorrer quando a estrutura está vazia e submetida à ação do vento no modo de flambagem assimétrico, ou seja, quando a solicitação que predomina é a pressão externa do vento. Observe-se a distribuição das tensões tangenciais nas figuras 49 e 50 (página 74), com atenção às regiões de concentração de tensão positiva e de tensão negativa. Esse modo de flambagem pode ocorrer na forma de ovalização da seção transversal da estrutura.

No modelo 5, admitidas também as condições de solicitação do vento e a estrutura vazia, a flambagem pode ocorrer devido à concentração de tensões de compressão de membrana (meridionais).

A figura 83 ilustra os casos do modelo 1 (quadros 1 e 2) e do modelo 5 (quadros 4,5 , 6). No modelo 1, as tensões que podem potencializar a flambagem são as tensões tangenciais, mas não há predomínio dessas tensões sobre as tensões meridionais de membrana. As tensões que predominam no modelo 5 são as tensões meridionais. 


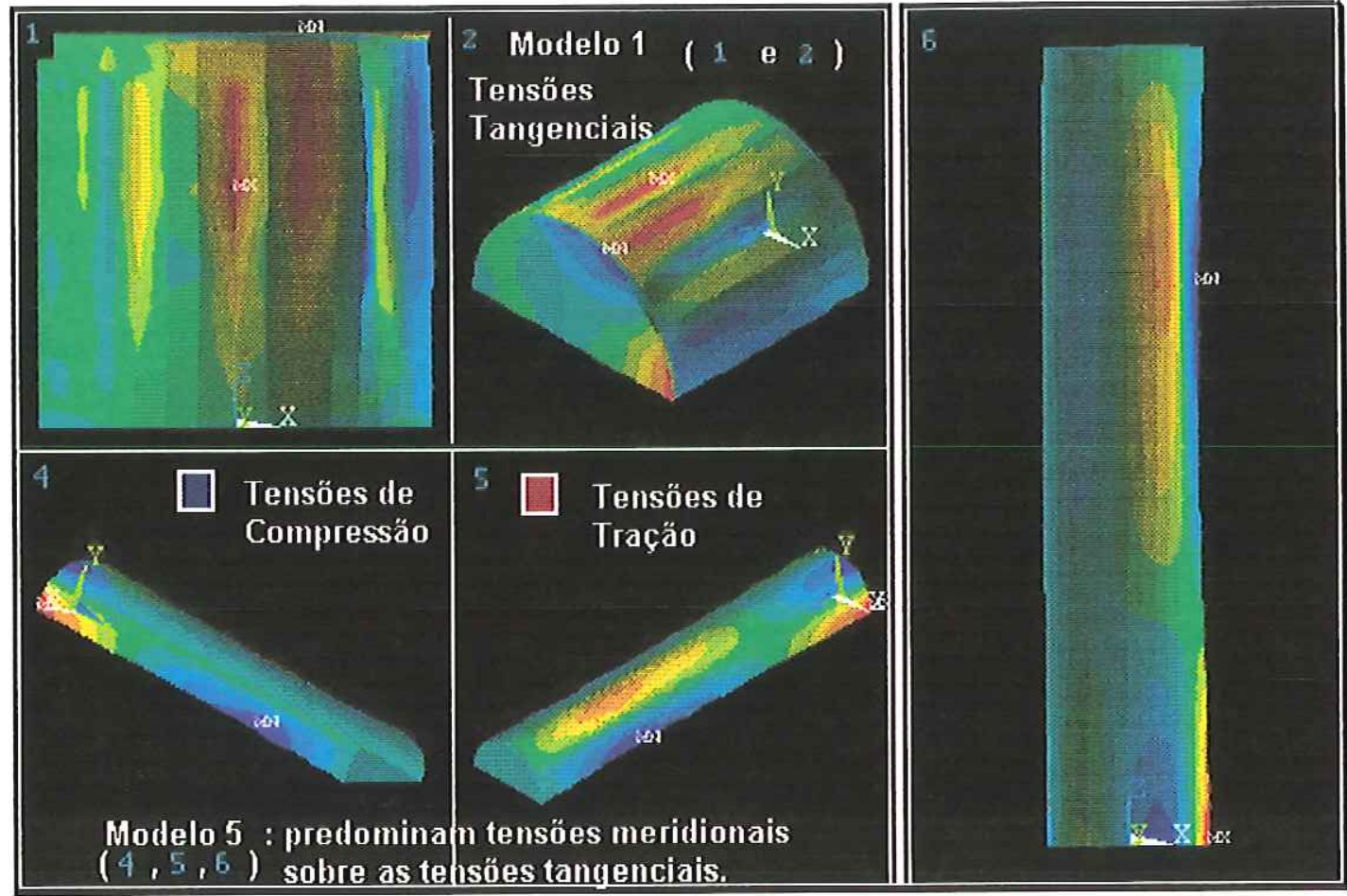

Figura 83 - Regiões de concentração de tensões nos modelos 1 e 5.

Quando a estrutura é um silo e a solicitação predominante deve-se ao material ensilado, o modo de flambagem é o axissimétrico. Os silos mais baixos $(\mathrm{H} / \mathrm{D}=1)$ apresentam menor risco de sofrer flambagem devido à compressão meridional (direção da geratriz) do que os silos de relação $\mathrm{H} / \mathrm{D}=5$, porque o atrito desenvolve-se por unidade de comprimento. Os resultados obtidos para o modelo 1 indicam maior concentração de tensão tangencial em relação à tensão meridional de compressão (Tabela 10, página 70 e gráficos 4 e 5, páginas 72 e 73). Para o modelo 5, as tensões tangenciais não predominam sobre as tensões meridionais (Tabela 12, página 82 e gráficos 9,10 , páginas 84 e 85 ).

Os resultados obtidos com a norma do ECCS (1988) referem-se ao exemplo 6 do capítulo 5. Os valores da análise numérica foram obtidos no ANSYS(C) para as solicitações do material ensilado, não sendo considerada a análise da solicitação do vento.

$\mathrm{Na}$ análise numérica, o silo apresentou valor de flambagem maior que o valor calculado com o ECCS(1988). O cálculo do silo, considerando a ação do material ensilado provocando compressão na direção da geratriz, forneceu resultados da tensão crítica praticamente iguais ao da teoria clássica de flambagem no caso axissimétrico (equação 44). O caso onde é considerada a ação de pressão lateral interna (provocada pelo material ensilado) forneceu uma tensão crítica de flambagem menor que o da equação 44. Os valores 
encontrados utilizando-se o $\operatorname{ECCS(1988)~são~menores~ainda~que~a~simulação~numérica~para~o~}$ caso de compressão axial combinado à pressão interna (em torno de $20 \%$ menores).

Pode-se observar que as discrepâncias na avaliação dos valores críticos da tensão são oriundas de fatores considerados apenas com parâmetros experimentais. A discrepância maior é obtida para o silo com material ensilado, onde a diferença entre o valor clássico da tensão crítica (eq. 44) e os valores numéricos via ANSYSC chegam a 30\%. Para os valores obtidos com o ECCS(1988), a tensão crítica chega a ser $50 \%$ menor que o valor obtido com a expressão clássica de compressão axissimétrica (eq. 44).

Como sugestões a trabalhos futuros, pode-se observar que os casos de estruturas danificadas pelo vento são numerosos e a bibliografia é reduzida para o estudo de estruturas submetidas à ação do vento. Um estudo para se calcular uma estrutura cilíndrica (silo ou reservatório metálico de paredes delgadas) submetida à ação do vento é necessário para se calcular esse tipo de estrutura.

A consideração de imperfeições também pode ser abordada em um estudo. Já existe uma bibliografía mais abrangente que o caso de solicitação de vento, com estudos de parâmetros para serem considerados esses efeitos devidos às imperfeições.

Como observação fínal, deve-se cuidar na simulação numérica de detalhes inerentes ao projeto em si. Não foram trabalhados detalhes de aberturas no costado, nem sistemas de ventilação interna do material ensilado. Os efeitos devidos ao esvaziamento do silo foram considerados nas ações, para um esvaziamento centrado, não sendo considerada uma descarga excêntrica na simulação numérica.; um caso que pode ser estudado. 


\section{REFERÊNCIAS BIBLIOGRÁFICAS}

ABNT - NBR 6120 (1980) Fcargas para o cálculo de estruturas de edificações

ABNT - NBR 6123 (1987) Forças Devidas ao Vento em Edificações. (incorporada a errata n 1 de DEZ./1990 - 84 pág)

ABNT - NBR 7821 (1983) - Tanques soldados para armazenamento de petróleo e derivados.

ABNT - NBR 8681 (1983) - Ações e segurança nas estruturas.

ABNT - NBR 8800 (1986) - Projeto e execução de estruturas de aço de edificios.

ABNT - NBR11162 (1990) - Silos cilíndricos para grãos vegetais.

ABNT - NBR11165 (1990) - Componentes de silos cilíndricos metálicos para grãos vegetais

ANSOURIAN, Peter (1992). "On the Buckling Analysis and Design of Silos and Tanks". Journal of Constructional Steel Research, 23, 21 pág.

AWWA (1967) - American Water Works Asociation: Steel Tanks, Standpipes, Reservoirs and Elevated Tanks - For Water Storage. AWWA D100-67 - AWS D5, 2-67; New York.

BEAUFAIT, Fred W., JOANNIDES, Socrates, A , GERLEIN, Mauricia A .(1967) "Analysis of Shell Walls of Circular Tanks under Axissummetrical Pressure".

BILLINGTON, David P (1965). Thin Shell Concrete Structures. McGraw-Hill, USA.

BULL, John W. (1990) Finite Element Applications to Thin-Walled Structures. ElsevierApplied Science, edited by John W. Bull, The Universities Press LTD, Belfast, Northern Ireland.

BRIASSOULIS, D., PECKNOLD, D. A (1986). “Anchorage Requirements for Wind-Loaded Empty Silos", Journal of Structural Engineering, vol 112, n².

BRUSH, D.O., ALMROTH, B. O. (1975) Buckling of Bars, plates and shells. McGraw-Hill Book company, New York

BUSHNELL, David . (1989) "Computerized Buckling analysis of shells. Kluwer Academic Publishers, Dordrecht, The Netherlands.

CROLL, J.G.A., WALKER, A. C. (1972) Elements of Structural Stability. The Macmillan Press, Great Britain.

DIN $1055-6$ (1986), Lastnnahmen für Bautenm Lasten in Silozellen. Fassung September .

DIN 18914, (1985) Thin walled cylindrical steel tower silos. 
EGGWERTZ, Sigge, SAMUELSON, Lars A. (1991) "Design of Shell Structures with Opening Subjected to Buckling". Journal of Construction Steel Research, 18.

ESSLINGER, M., AHMED, S. R., SCHROEDER, H. H. (1971) "Stationäre Windbelastung Offner und Geschlossener Kreyszylinder Silos, Der Stahlbau, Dec.

FORTES FILHO, Jorge.(1985) Uma Introdução ao Estudo dos Silos. Dissertação de mestrado na Escola de Engenharia de São Carlos/USP.

FARKAS, Jósef, JARMÁI, Károly.(1996) "Fabrication Cost Calculation and Optimum Design of Welded Steel Silos". Welding in the World - Le Soudage dans Le Monde. Vol 37, No 5 , Elsevier Science Ltd, Great Britain, 1996.

GAYLORD, Edwin Henry, GAYLORD, Charles N (1984). Design of Steel Bins for Storage of Bulk Solids. New Jersey, USA, William J. Hall (editor).

GAYLORD, Edwin Henry, GAYLORD, Charles N.(1977) "Granular Material Pressures in Bins", Winter Meeting American Society of Agricultural Engineers. ( Paper nº77-4503 ASAE).

GHALI, A .(1979) Circular Storage Tanks and Silos. London, 1st edition, Spon Limited.

GODOY, Luis A .(1996) Thin-Walled Structures with Structural Imperfections: Analysis and Behavior, Pergamon

GRAVINA, Pedro B. J.(1957) Teoria e Cálculo das Cascas. Cascas de Revolução. São Paulo.

GREINER, R., DERLER, P.(1995) "Effect of Imperfections on Wind Loaded Cylindrical Shells". Thin-Walled Structures.

HUTCHINSON, John. (1965) "Axial Buckling of Pressurized Imperfect Cylindrical Shells". AIAA Journal, vol 3, $n^{\circ} 8$.

KNEBEL, K., SCHWEIZERHOF, K (1995). "Buckling of Cylindrical Shells Containing Granular Solids". Thin-Walled Structures, 23, 1995.

KNOEDEL, Peter, UMMENHOFER, Thomas, SCHULZ, Ulrich (1995). "On the Modelling of Different Types of Imperfections in Silo Shells". Thin-Walled Structures, 23.

KWADE, Arno, SCHULZE, Dietmar, SCHWEDES, Jörg.(1994) "Auslegung von Silos: Unmittelbare Messung des Horizontallastverjhältnisses". Beton und Stahlbetonbau 89 , Heft 3 - Ernst \& Sohn

LAMBERT, F. W., The Theory and Practical Design of Bunkers. The British Constructional Stellwork Association LTD, Hancock House, 87, Vincent Square, London, SW1

MACDONALD, P.A , HOLMES, J. D., KWOK, K.C.S (1990). "Wind Loads on circular storage bins, silos and tanks. Part III. Fluctuating and Peak Pressure Distributions".

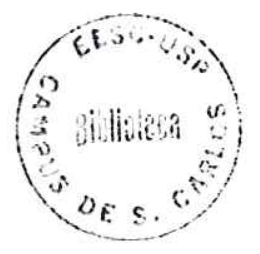


Journal of Wind Engineering and Structural Aerodynamics. 34 319-337. Elsevier Science Publishers B.V., Amsterdam.

MADERSPACH, V., GAUNT, J.T., SWORD, J. II. (1973)“Buckling of Cylindrical Shells due to Wind Loading". Der Stahlbau 9, 269-277.

MANUAL BRASILEIRO PARA CÁLCULO DE ESTRUTURAS METÁLICAS (1986), Vol II, Brasília/DF, MIC/STI.

PIROK, J.N., WOZNIAK, R.S.( 1989) "Steel Tanks". McGraw-Hill Book Company.

ROTTER, J.M., JUMIKIS, P.T., FLEMING, S.P. (1989) "Experiments on the Buckling of Thinwalled Model Silo Structures". Journal Of Construction Steel Research 13

TIMOSHENKO, Stephen P., GERE, James M.(1961) Theory of Elastic Stability. Tokyo, McGrawHill Kogakusha, 2nd ed.

TIMOSHENKO, Stephen P. (1940) Theory of Plates and Shells. McGraw-Hill Book Company, Inc, New York.

TRAHAIR, N.S. (1985) "Structural Design Criteria for Steel Bins and Silos". International Journal of Bulk Solids Storage in Silos. Vol $01, \mathrm{n}^{\circ} 2$.

TRAHAIR, N.S.et al. (1983) "Structural Design of Steel Bins for Bulk Solids". Australian Institute of Steel Construction, Nov. 


\section{ANEXOS}

ANEXO 1 - Valores de Cálculo para Materiais Ensilados ( Tabela 1 da DIN1055-6 (1986)).

ANEXO 2 - Tabelas para Cálculo de Placas - Teoria de Grandes Deflexões.

ANEXO 3 - Isopletas da Velocidade Básica $V_{0}(\mathrm{~m} / \mathrm{s})$ ( Tabela 1 da NBR 6123 (1987) ).

ANEXO 4 - Cálculo das Áreas de Fatias da Superfície Cônica.

ANEXO 5 - Tabelas de Perfis do Manual Brasileiro para Cálculo de Estruturas Metálicas.

ANEXO 6 - Demonstração das Fórmulas dos Regimes de Membrana e de Flexão. 


\section{ANEXO 1}

Valores de Cálculo para Materiais Ensilados

( Tabela 1 da DIN1055-6 (1986) ) 


\begin{tabular}{|c|c|c|c|c|c|c|c|c|c|c|c|c|c|}
\hline 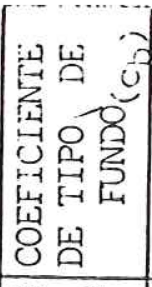 & $\underset{r-1}{N}$ & $\hat{r}$ & $\underset{r}{n}$ & ${ }_{r-1}^{m}$ & $\stackrel{\sim}{r-1}$ & $\tilde{r}$ & $\tilde{\tau}$ & $\begin{array}{l}? \\
\therefore i\end{array}$ & $\because$ & $r$ & $\tilde{r}$ & $\ddot{m}$ & $\rightarrow$ \\
\hline 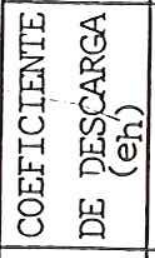 & $\begin{array}{l}0 \\
\text { in } \\
\text {-1 }\end{array}$ & $\begin{array}{l}0 \\
\text { I } \\
\text {-1 }\end{array}$ & $\begin{array}{r}\stackrel{\circ}{m} \\
\text {-1 }\end{array}$ & $\stackrel{\circ}{r}$ & $\stackrel{\circ}{\stackrel{\circ}{r}}$ & $\begin{array}{r}\stackrel{0}{m} \\
\text { r }\end{array}$ & $\underset{\sim}{\stackrel{O}{N}}$ & $\begin{array}{r}\stackrel{\circ}{m} \\
-1\end{array}$ & $\begin{array}{l}0 \\
= \\
r-1\end{array}$ & $\begin{array}{l}\circ \\
\text { m } \\
\text { ri }\end{array}$ & $\begin{array}{l}0 \\
\pm \\
-1\end{array}$ & $\underset{r-1}{\stackrel{O}{\sim}}$ & $\underset{r}{\stackrel{0}{\sim}}$ \\
\hline $\begin{array}{ll}\mu & \\
A & m \\
\mu & \beth^{m} \\
\alpha & \end{array}$ & $\stackrel{\sim}{\sim}$ & $\underset{0}{\sim}$ & $\stackrel{\sim}{\sim}$ & $\stackrel{\sim}{\sim}$ & $\begin{array}{l}\text { 음 } \\
\text { in } \\
0\end{array}$ & $\begin{array}{l}\text { 음 } \\
\text { in }\end{array}$ & $\begin{array}{l}\text { O } \\
\text { in } \\
0\end{array}$ & $\begin{array}{l}\text { O } \\
\text { م } \\
\text { م }\end{array}$ & 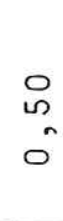 & $\stackrel{\circ}{\text { m }}$ & $\begin{array}{l}n \\
\text { J } \\
0 \\
0\end{array}$ & $\begin{array}{l}0 \\
\text { 品 } \\
0 \\
0\end{array}$ & $\begin{array}{l}n \\
\text { J } \\
0\end{array}$ \\
\hline $\begin{array}{ll}0 & \\
1 & 2 \\
0 & 2\end{array}$ & $\begin{array}{l}0 \\
\text { o } \\
0 \\
0\end{array}$ & $\begin{array}{l}0 \\
\text { J } \\
0 \\
0\end{array}$ & $\begin{array}{l}n \\
m \\
0\end{array}$ & $\stackrel{n}{m}$ & in & $\begin{array}{l}\circ \\
0 \\
0 \\
0\end{array}$ & $\begin{array}{l}\text { o } \\
\text { in } \\
\text { on }\end{array}$ & $\begin{array}{l}0 \\
\text { I } \\
0 \\
0\end{array}$ & $\begin{array}{l}\text { in } \\
\text { in } \\
0\end{array}$ & $\begin{array}{l}0 \\
\text { I } \\
0 \\
0\end{array}$ & $\begin{array}{l}\text { 음 } \\
\text { on }\end{array}$ & $\begin{array}{l}0 \\
\text { I } \\
0 \\
0\end{array}$ & \begin{tabular}{l}
$n$ \\
\multirow{J}{*}{} \\
0
\end{tabular} \\
\hline $\begin{array}{ll}H & \\
\alpha & -1 \\
H & Z^{-1} \\
4 & \end{array}$ & $\begin{array}{l}\circ \\
0 \\
0 \\
0\end{array}$ & $\begin{array}{l}0 \\
0 \\
0 \\
0\end{array}$ & $\begin{array}{l}0 \\
\text { in } \\
0\end{array}$ & $\begin{array}{l}0 \\
\text { 으 } \\
0\end{array}$ & $\begin{array}{l}n \\
n \\
0 \\
0\end{array}$ & $\stackrel{n}{n}$ & $\begin{array}{l}\circ \\
0 \\
0 \\
0\end{array}$ & $\begin{array}{l}\text { ㅇ } \\
\text { nn } \\
0\end{array}$ & $\begin{array}{l}n \\
0 \\
0 \\
0\end{array}$ & $\begin{array}{l}\text { n } \\
\text { in } \\
0\end{array}$ & $\begin{array}{l}0 \\
0 \\
0 \\
0\end{array}$ & $\begin{array}{l}\circ \\
\text { in } \\
0 \\
0\end{array}$ & 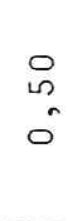 \\
\hline $\begin{array}{r}\theta \\
E \\
0 \\
<0 \\
1 \\
-1\end{array}$ & \begin{tabular}{l}
$\infty$ \\
\multirow{J}{*}{} \\
0
\end{tabular} & \begin{tabular}{l}
$\infty$ \\
\multirow{J}{*}{} \\
0
\end{tabular} & $\begin{array}{l}0 \\
\text { nn } \\
0\end{array}$ & $\begin{array}{l}0 \\
\text { m } \\
0\end{array}$ & กั & $\begin{array}{l}\text { न1 } \\
\exists \\
0\end{array}$ & \begin{tabular}{l}
$\infty$ \\
\multirow{J}{*}{} \\
0
\end{tabular} & $\begin{array}{l}\text { 음 } \\
\text { on }\end{array}$ & \begin{tabular}{l}
0 \\
\multirow{J}{0}{} \\
0 \\
0
\end{tabular} & $\begin{array}{l}m \\
\text { in } \\
0 \\
0\end{array}$ & $\begin{array}{l}\infty \\
\pm \\
0 \\
0\end{array}$ & $\begin{array}{l}n \\
\text { n } \\
0\end{array}$ & مn \\
\hline 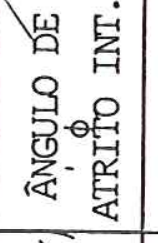 & $\stackrel{-1}{m}$ & $\mathrm{~m}^{-1}$ & $\stackrel{\bullet}{\sim}$ & $\tilde{\sim}$ & $\stackrel{\sim}{\sim}$ & $\stackrel{\omega}{m}$ & $\stackrel{\vec{m}}{m}$ & $\stackrel{\circ}{m}$ & $\stackrel{m}{m}$ & $\stackrel{\infty}{\sim}$ & $\vec{m}$ & $\grave{\sim}$ & $\lesssim$ \\
\hline 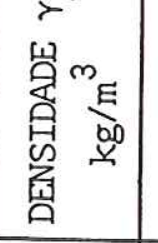 & $\begin{array}{l}\circ \\
\text { 으 }\end{array}$ & $\begin{array}{l}\circ \\
\circ \\
\infty\end{array}$ & $\begin{array}{l}\circ \\
\circ \\
\infty\end{array}$ & 음 & $\begin{array}{l}0 \\
\vec{a} \\
\text { वे }\end{array}$ & $\begin{array}{l}\circ \\
0 \\
0 \\
\text { ஸे } \\
-1\end{array}$ & $\begin{array}{l}\circ \\
\circ \\
\infty \\
-1\end{array}$ & $\begin{array}{l}\text { ㅇ } \\
\text { m } \\
\text { r- }\end{array}$ & $\begin{array}{l}\text { ○ } \\
\infty \\
\infty \\
-1 \\
-1\end{array}$ & $\begin{array}{l}0 \\
0 \\
0 \\
-1 \\
-1\end{array}$ & $\begin{array}{l}\circ \\
\circ \\
0\end{array}$ & 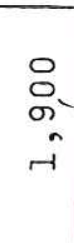 & $\begin{array}{l}\stackrel{\circ}{\circ} \\
\stackrel{-}{n} \\
-1\end{array}$ \\
\hline 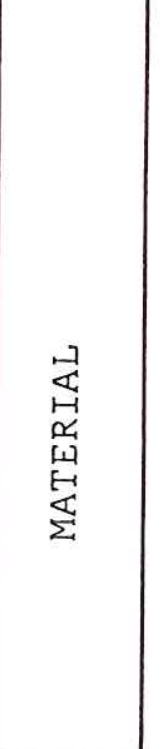 & 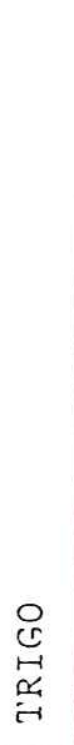 & 옥 & 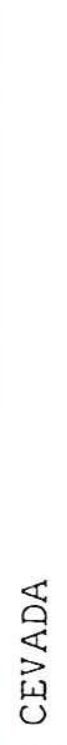 & 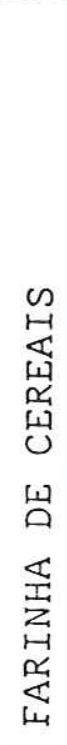 & 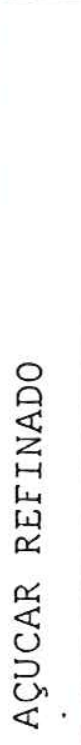 & 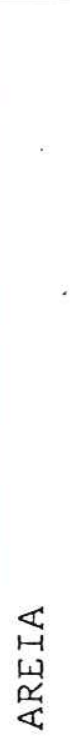 & 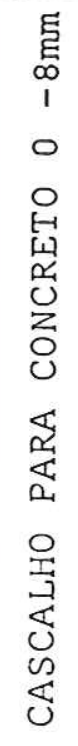 & 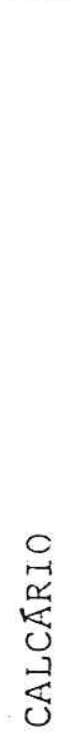 & 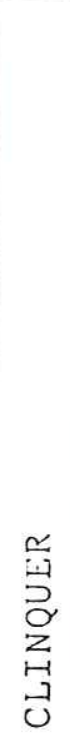 & 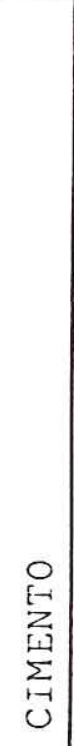 & 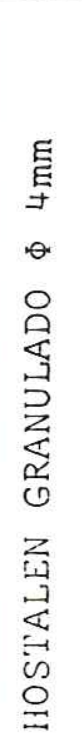 & 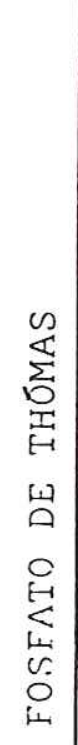 & 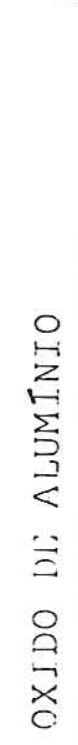 \\
\hline
\end{tabular}




\section{Schüttgutlasten}

\subsection{Einflußgrößen}

\subsubsection{Schüttguteigenschaften}

Die Rechenwerte für die Eigenschaften körniger und staubförmiger Schüttgüter sind in Tabelle 1 angegeben. Sie dürfen vereinfachend über die Siloguttiefe $z$ konstant angenommen werden.

Tabelle 1. Rechenwerte für Schüttgüter

\begin{tabular}{|c|c|c|c|c|c|c|c|}
\hline Schüttgut & $\begin{array}{l}\text { Wichte } \gamma \\
\mathrm{kN} / \mathrm{m}^{3}\end{array}$ & $\begin{array}{l}\text { Horizontal- } \\
\text { lastver- } \\
\text { hältnis } \\
\quad \lambda\end{array}$ & $\begin{array}{l}\text { Wandre } \\
\qquad \mu_{1}\end{array}$ & ungsbe & $\begin{array}{l}\text { werte } \\
\mu_{3}\end{array}$ & $\begin{array}{l}\text { Entlee- } \\
\text { rungs- } \\
\text { faktor } \\
\mathrm{e}_{\mathrm{h}}\end{array}$ & $\begin{array}{l}\text { Schütt- } \\
\text { gutbei- } \\
\text { wert } \\
\beta_{G}\end{array}$ \\
\hline Weizen & 9,0 & 0,60 & 0,60 & 0,40 & 0,25 & 1,4 & 0,5 \\
\hline Ma is & 8,0 & 0,60 & 0,60 & 0,40 & 0,25 & 1,6 & 0,9 \\
\hline Braugerste & 8,0 & 0,65 & 0,50 & 0,35 & 0,25 & 1,4 & 0,5 \\
\hline Getreidemeh 1 & 7,0 & 0,40 & 0,50 & 0,35 & 0,25 & 1,4 & 0,6 \\
\hline Weißzucker & 9,5 & 0,60 & 0,55 & 0,50 & 0,45 & 1,2 & 0,4 \\
\hline $\begin{array}{l}\text { Quarzsand } \\
\text { gebrochen }\end{array}$ & 16,0 & 0,50 & 0,60 & 0,50 & 0,40 & 1,4 & 0,4 \\
\hline Betonkies & 18,0 & 0,60 & 0,60 & 0,50 & 0,40 & 1,3 & 0,4 \\
\hline Kalksteinmehl & 13,0 & 0,65 & 0,55 & 0,50 & 0,40 & 1,2 & 0,5 \\
\hline Zementkl inker & 18,0 & 0,50 & 0,60 & 0,55 & 0,45 & 1,2 & 0,7 \\
\hline Zement & 16,0 & 0,65 & 0,50 & 0,45 & 0,40 & 1,2 & 0,5 \\
\hline Aluminiumoxid & 12,0 & 0,65 & 0,50 & 0,45 & 0,40 & 1,2 & 0,5 \\
\hline Thomasphosphat & 22,0 & 0,65 & 0,55 & 0,50 & 0,40 & 1,3 & 0,5 \\
\hline
\end{tabular}

\subsubsection{Form und Größe des Zellenquerschnitts}

Die Form und Größe des Zellenquerschnittes wird im allgemeinen durch das Verhältnis von Fläche zu Umfang $A / u$ berücksichtigt.

Für den Sonderfall der Spaltzelle gilt $A / u=b / 2$ mit $b$ nach Bild 2. Für den Sonderfall der Zwickelzelle gilt $A / U=d / 4$ mit $d$ nach Bild 3 . 


\section{ANEXO 2}

TABELAS PARA

\section{CÁLCULO DE PLACAS TEORIA DE GRANDES DEFLEXÕES}

Extraídas de Gaylord\&Gaylord (1984) 
TABLE 6-5. UNIFORMLY LOADED RECTANGULAR PLATES WITH SIMPLY SUPPORTEO EOGES: LARGE.DEFLECTION THEORY: $A B \geqslant 2$

\begin{tabular}{|c|c|c|c|c|}
\hline$\frac{b}{t} \vee i \frac{f}{E}$ & $\frac{p E}{f^{2}}$ & $\sqrt{\frac{p}{E}}\left(\frac{b}{t}\right)^{2}$ & $\frac{f_{g}}{f}$ & $\frac{8}{1}$ \\
\hline 1.5 & 0.702 & 1.89 & 0.159 & 0.363 \\
\hline 1.75 & 0.594 & 2.36 & 0.199 & 0.472 \\
\hline 2 & 0.535 & 2.93 & 0.239 & 0.591 \\
\hline 2.25 & 0.504 & 3.59 & 0.279 & 0.713 \\
\hline 2.5 & 0.488 & 4.37 & 0.315 & 0.848 \\
\hline 2.75 & 0.479 & 5.23 & 0.352 & 0.980 \\
\hline 3 & 0.475 & 6.20 & 0.386 & 1.12 \\
\hline 3.5 & 0.469 & 8.39 & 0.446 & 1.40 \\
\hline 4 & 0.462 & 10.9 & 0.497 & 1.70 \\
\hline 4.5 & 0.455 & 13.7 & 0.542 & 1.99 \\
\hline 5 & 0.444 & 16.7 & 0.580 & 2.28 \\
\hline 5.5 & 0.432 & 19.9 & 0.613 & 2.56 \\
\hline 6 & 0.419 & 23.3 & 0.641 & 2.35 \\
\hline 7 & 0.393 & 30.7 & 0.685 & 3.44 \\
\hline 8 & 0.368 & 38.8 & 0.719 & 4.02 \\
\hline 9 & 0.342 & 47.4 & 11.748 & 4.59 \\
\hline 10 & 0.323 & 56.8 & 0.770 & 5.19 \\
\hline 11 & 0.303 & 66.6 & 0.789 & $5.7 ?$ \\
\hline 12 & 0.286 & 77.0 & 0.814 & 6.36 \\
\hline 13 & 0.271 & 88.0 & 0.818 & 6.99 \\
\hline 14 & 0.257 & 99.4 & 0.830 & 7.55 \\
\hline 15 & 0.244 & 111.1 & 0.841 & 8.13 \\
\hline 16 & 0.232 & 123.3 & 0.850 & 8.71 \\
\hline 17 & 0.222 & 136.2 & 0.857 & 9.32 \\
\hline 18 & 0.212 & 149.2 & 0.865 & 9.90 \\
\hline
\end{tabular}

Chep. 6 Design of Structurat Components

TABLE 6-6. UNIFORMLY LOADED RECTANGULAR PLATES WITH MOMENT.RESISTANT EDGES: LARGE.DEFLECTION THEORY: $a \beta>1.5$

\begin{tabular}{|c|c|c|c|c|}
\hline$\frac{b}{t} \vee \frac{f}{E}$ & $\frac{p E}{f^{2}}$ & $\frac{\bar{n}}{\ddot{n}}\left(\begin{array}{l}b \\
t\end{array}\right)^{2}$ & $\frac{f}{f}$ & $\stackrel{s}{\dddot{t}}$ \\
\hline 1.50 & 0.880 & 2.11 & $0 .(1) 9$ & 0.116 \\
\hline 1.75 & 0.645 & 2.46 & 0.025 & 0.169 \\
\hline 2 & 0.497 & 2.82 & 0.032 & 0.223 \\
\hline 2.25 & 0.393 & 3.17 & 0.040 & 0.273 \\
\hline 2.5 & 0.321 & 3.54 & 0.047 & 0.329 \\
\hline 2.75 & 0.268 & 3.92 & 0.055 & 0.393 \\
\hline 3 & 0.229 & 4.31 & 0.062 & 0.458 \\
\hline 3.5 & 0.175 & 5.12 & 0.078 & 0.588 \\
\hline 4 & 0.141 & 6.01 & 0.090 & 0.737 \\
\hline 4.5 & 0.118 & 6.96 & 0.102 & 0.882 \\
\hline 5 & 0.101 & 7.95 & 0.112 & 1.02 \\
\hline 5.5 & 0.0890 & 9.02 & 0.120 & 1.17 \\
\hline 6 & 0.0794 & 10.1 & 0.127 & 1.21 \\
\hline 7 & 0.0655 & 12.5 & 0.137 & 1.59 \\
\hline 8 & 0.0558 & 15.1 & 0.144 & 1.88 \\
\hline 9 & 0.0486 & 17.9 & 0.147 & 2.18 \\
\hline 10 & 0.0431 & 20.8 & 0.150 & 2.47 \\
\hline 11 & 0.0386 & 23.8 & 0.154 & 2.74 \\
\hline 12 & 0.0350 & 26.9 & 0.156 & 3.01 \\
\hline 13 & 0.0320 & 30.2 & 0.157 & 3.29 \\
\hline 14 & 0.0295 & 33.7 & 0.158 & 3.59 \\
\hline 15 & 0.0274 & 37.2 & 0.159 & 3.87 \\
\hline 16 & 0.0254 & 40.8 & 0.159 & 4.15 \\
\hline 17 & 0.0239 & 44.7 & 0.159 & 4.45 \\
\hline 18 & 0.0224 & 48.5 & $0.16^{\circ}$ & 4.72 \\
\hline
\end{tabular}


ANEXO 3

Isopletas da Velocidade Básica $\mathrm{V}_{0}(\mathrm{~m} / \mathrm{s})$

( Tabela 1 da NBR 6123 (1987) ) 


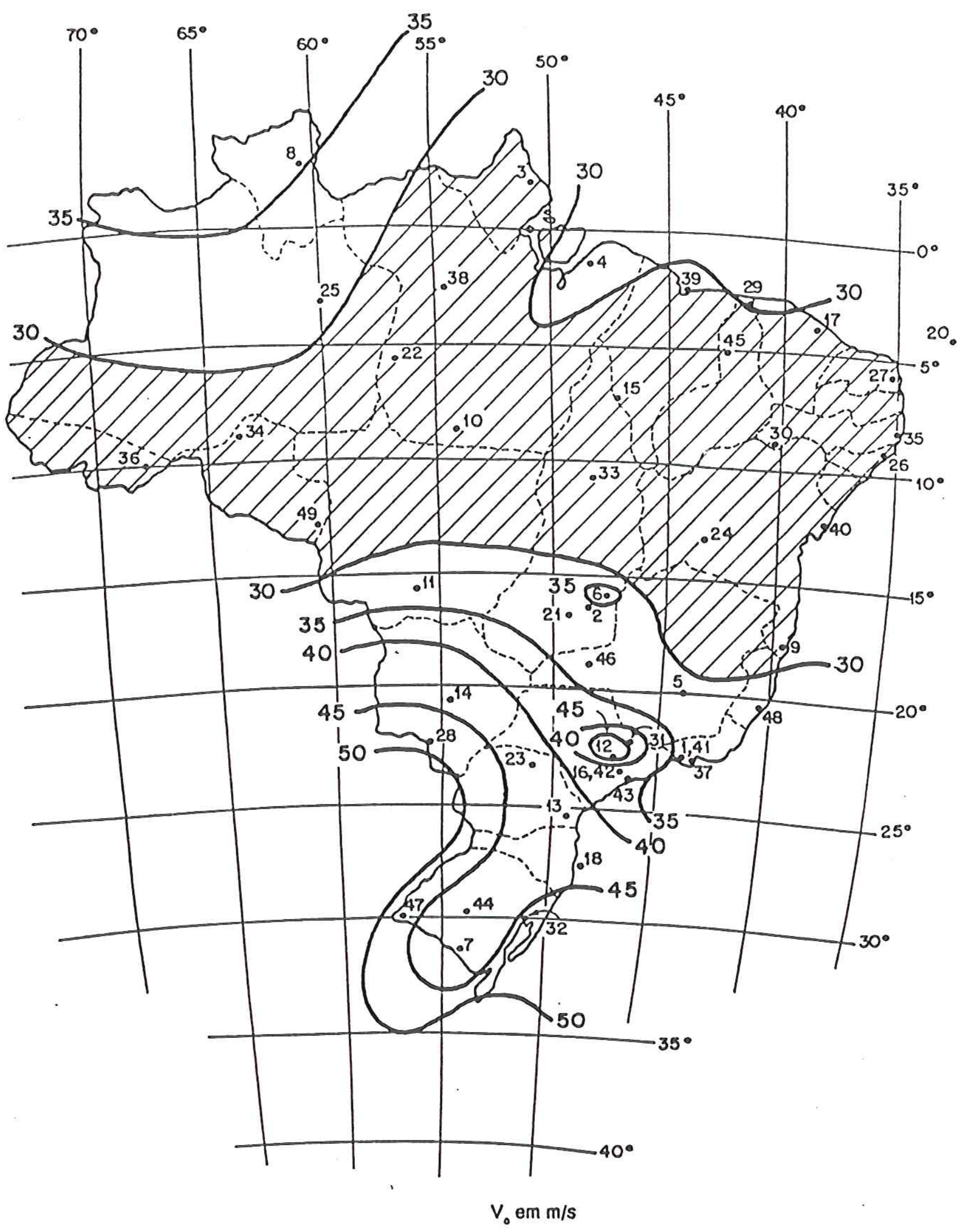

$V_{0}$ : máxima velocidado média medida sobre 3s, que pode ser excedida em média uma vez em 50 anos, a $10 \mathrm{~m}$ sobre o nivel do terreno em lugar aberto e plano 
ANEXO 4 


\section{Cálculo das Áreas de Fatias da Superfície Cônica}

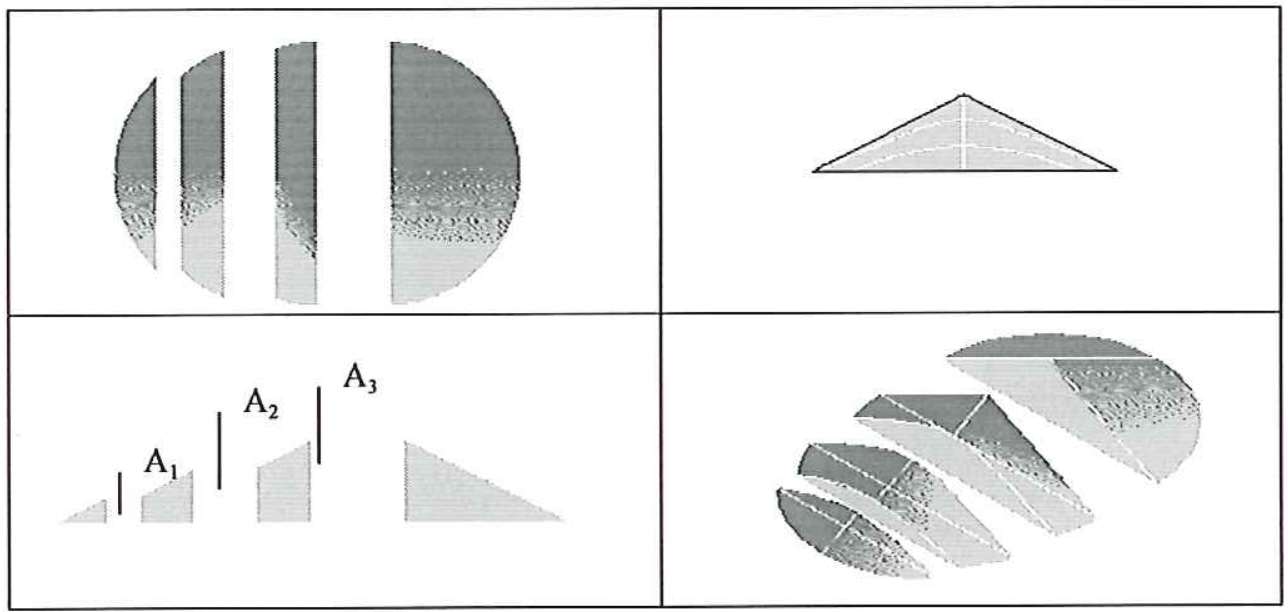

Figura 1 - Vistas de um Cone Fatiado

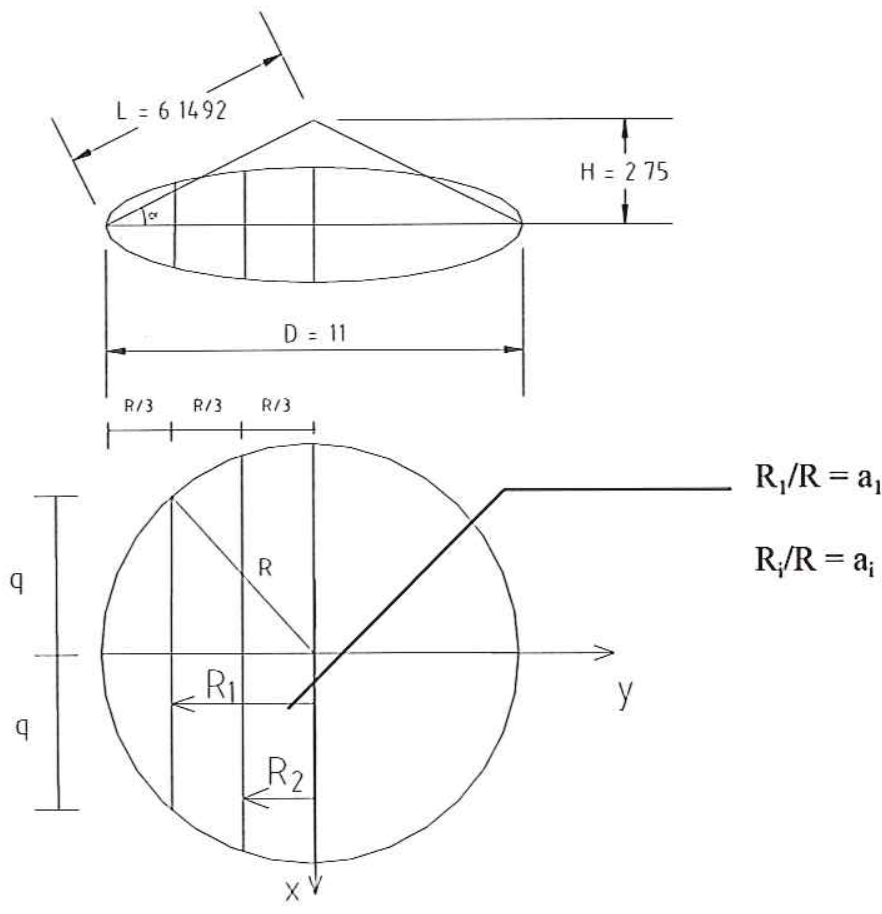

Figura 2 - Dimensões e Nomenclatura

$\mathrm{O}$ objetivo é calcular as áreas $\mathrm{A}_{1}, \mathrm{~A}_{2}, \mathrm{~A}_{3}$ indicadas na figura 1 . As áreas são superfícies cuja projeção R é uma curva diferenciável (um segmento de círculo), logo aplicase o cálculo diferencial com a fórmula extraída de SWOKOWSKI, Earl William. (Cálculo com Geometria Analítica, vol. 2, Mc Graw-Hill do Brasil, São Paulo, 1983) :

$A=\iint_{R} \sqrt{\left(f_{x}(x, y)\right)^{2}+\left(f_{y}(x, y)\right)^{2}+1} d A$ 
Onde $f_{x}$ é a derivada parcial em relação a $x$ e $f_{y}$ é a derivada parcial em relação a y. A função $f$ da superfície do cone é dada em função de $x$ e de $y$, tal que:

$f(x, y)=z \Rightarrow(2 z-5,5)^{2}=x^{2}+y^{2}$

$z=\frac{\sqrt{x^{2}+y^{2}}+5,5}{2}$

$f_{x}(x, y)=\frac{1}{2} \frac{x}{\sqrt{x^{2}+y^{2}}}$

$f_{y}(x, y)=\frac{1}{2} \frac{y}{\sqrt{x^{2}+y^{2}}}$

Aplicando a equação 1 :

$A=\iint_{R}^{\sqrt{5}} \frac{\sqrt{2}}{2} d x d y$

Os limites de integração serão aplicados para a dedução de uma fórmula geral para o cálculo de uma área de forma semelhante à área $A_{1}$. Para tanto, são definidos os intervalos:

$-\sqrt{R^{2}-y^{2}} \leq x \leq 0 \quad R_{i} \leq y \leq R$

Substituindo os intervalos de x e de y na equação 2: $\quad \frac{A}{2}=\frac{\sqrt{5}}{2} \int_{R_{i}}^{R} \int_{-\sqrt{x^{2}-y^{2}}}^{0} d x d y$

$\left.\frac{A}{2}=\frac{\sqrt{5}}{2} \int_{R_{i}}^{R} 0-\left(-\sqrt{R^{2}-y^{2}}\right)\right) d y \Rightarrow A=\frac{\sqrt{5}}{2}\left(\frac{y}{2} \sqrt{R^{2}-y^{2}}+\left.\frac{R^{2}}{2} \operatorname{arcsen}\left(\frac{y}{R}\right)\right|_{R_{i}} ^{R}\right.$

$A=\sqrt{5} R^{2}\left(\frac{\pi}{2}-\frac{a}{2} \sqrt{1-a^{2}}+\frac{R^{2}}{2} \operatorname{arcsen}(a)\right)$

A equação 3 é a fórmula para o cálculo de áreas semelhantes à área $A_{1}$ da figura 1 .

Para as dimensões indicadas na figura 2, as áreas resultam:

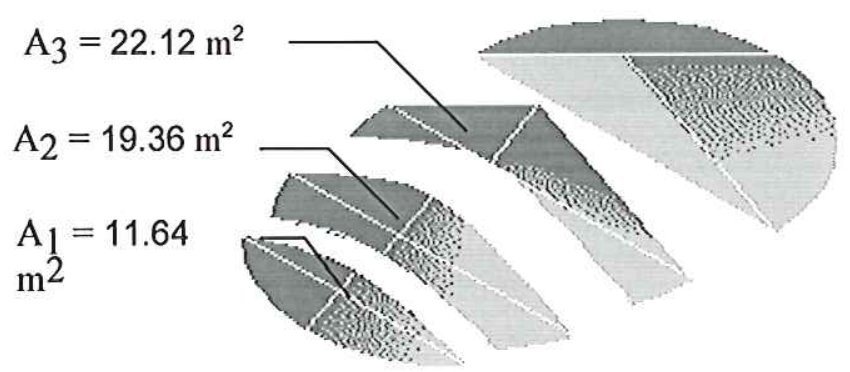

Figura 3 - Áreas da superfície cônica 


\title{
ANEXO 5
}

TABELAS DE PERFIS DO

\author{
MANUAL BRASILEIRO \\ PARA CÁLCULO DE \\ ESTRUTURAS METÁLICAS
}

VOLUME II

1986

MINISTÉRIO DA INDÚSTRIA E DO COMÉRCIO

SECRETARIA DE TECNOLOGIA INDUSTRIAL 


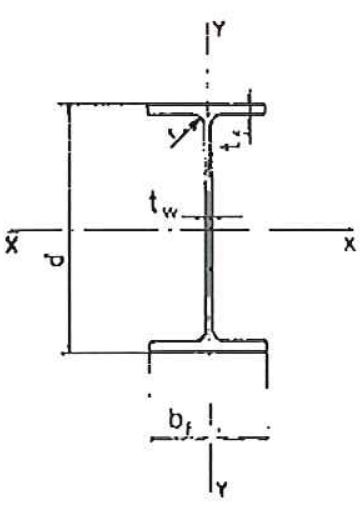

PERFIS I DE ABAS PARALLLLAS,

DE AC (), LAMINADOS

CONF, NBR 6009/80

PROPRILDADES PARA PROJETO

\begin{tabular}{|c|c|c|c|c|c|c|c|c|c|c|c|c|c|}
\hline \multirow{4}{*}{$\begin{array}{c}\text { DESIG. } \\
\text { IP }\end{array}$} & \multirow{2}{*}{\multicolumn{5}{|c|}{ DIMENSŐES EM mm }} & \multirow{4}{*}{$\begin{array}{l}\mathrm{A} \\
\mathrm{cm}^{2}\end{array}$} & \multirow{4}{*}{$\frac{\mathrm{P}}{\mathrm{Kg} / \mathrm{m}}$} & \multicolumn{6}{|c|}{ PROPRIEDADES ELÁSTICAAS } \\
\hline & & & & & & & & \multicolumn{3}{|c|}{ EIXO X - X } & \multicolumn{3}{|c|}{ EIXO Y - Y } \\
\hline & \multirow[b]{2}{*}{ d } & \multirow[b]{2}{*}{$b_{f}$} & \multirow[b]{2}{*}{$t_{w}$} & \multirow[b]{2}{*}{$t_{f} f$} & \multirow{2}{*}{$\mathbf{r}$} & & & $\mathrm{I}_{\mathrm{X}}$ & $\mathrm{w}_{\mathrm{x}}$ & $\mathbf{r}_{\mathbf{X}}$ & $\mathrm{I}_{\mathrm{y}}$ & $W_{y}$ & $\mathrm{ry}_{\mathrm{y}}$ \\
\hline & & & & & & & & $\mathrm{cm}^{4}$ & $\mathrm{~cm}^{3}$ & $\mathrm{~cm}$ & $\mathrm{~cm}^{4}$ & $\mathrm{~cm}^{3}$ & $\mathrm{~cm}$ \\
\hline 80 & 80 & 46 & 3,8 & 5,2 & 5 & 7,64 & 6,0 & 80 & 20,0 & 3,24 & 8,49 & 3,69 & 1,05 \\
\hline 100 & 100 & 55 & 4,1 & 5,7 & 7 & 10,3 & 8,1 & 171 & 34,2 & 4,07 & 15,9 & 5,79 & 1,24 \\
\hline 120 & 120 & 64 & 4,4 & 6,3 & 7 & 13,2 & 10,4 & 318 & 53,0 & 4,90 & 27,7 & 8,65 & 1,45 \\
\hline 140 & 140 & 73 & 4,7 & 6,9 & 7 & 16,4 & 12,9 & 541 & 77,3 & 5,74 & 44,9 & 12,3 & 1,65 \\
\hline 160 & 160 & 82 & 5,0 & 7,4 & 9 & 20,1 & 15,8 & 869 & 109 & 6,58 & 68,3 & 16,7 & 1,84 \\
\hline 180 & 180 & 91 & 5,3 & 8,0 & 9 & 23,9 & 18,8 & 1320 & 146 & 7,42 & 101 & 22,2 & 2,05 \\
\hline 200 & 200 & 100 & 5,6 & 8,5 & 12 & 28,5 & 22,4 & 1940 & 194 & 8,26 & 142 & 28,5 & 2,24 \\
\hline 220 & 220 & 110 & 5,9 & 9,2 & 12 & 33,4 & 26,2 & 2770 & 252 & 9,11 & 205 & 37,3 & 2,48 \\
\hline 240 & 240 & 120 & 6,2 & 9,8 & 15 & 39,1 & 30,7 & 3890 & 324 & 9,97 & 284 & 47,3 & 2,69 \\
\hline 270 & 270 & 135 & 6,6 & 10,2 & 15 & 45,9 & 36,1 & 5790 & 429 & 11,2 & 420 & 62,2 & 3,02 \\
\hline 300 & 300 & 150 & 7,1 & 10,7 & 15 & 53,8 & 42,2 & 8360 & 557 & 12,5 & 604 & 80,5 & 3,35 \\
\hline 330 & 330 & 160 & 7,5 & 11,5 & 18 & 62,6 & 49,1 & 11770 & 713 & 13,7 & 788 & 98,5 & 3,55 \\
\hline 360 & 360 & 170 & 8,0 & 12,7 & 18 & 72,7 & 57,1 & 16270 & 904 & 15,0 & 1040 & 123 & 3,79 \\
\hline 400 & 400 & 180 & 8,6 & 13,5 & 21 & 84,5 & 66,3 & 23130 & 3160 & 16,5 & 1320 & 146 & 3,95 \\
\hline 450 & 450 & 190 & 9,4 & 14,6 & 21 & 98,8 & 77,6 & 33740 & 1500 & 18,5 & 1680 & 176 & 4,12 \\
\hline 500 & 500 & 200 & 10,2 & 16,0 & 21 & 116 & 90,7 & 48200 & 1930 & 20,4 & 2140 & 214 & 4,31 \\
\hline 550 & 550 & 210 & 13,1 & 17,2 & 24 & 134 & 106 & 67120 & 2440 & 22,3 & 2670 & 254 & 4,45 \\
\hline 600 & 600 & 220 & 12,0 & 19,0 & 24 & 156 & 122 & 92080 & 3070 & 24,3 & 3390 & 308 & 4,46 \\
\hline
\end{tabular}




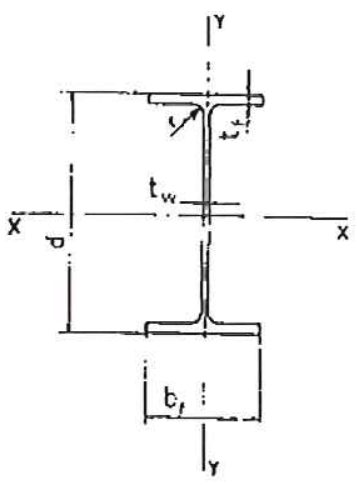

PEREIS I DF, ABAS PARALELAS, DF. AÇO, LAMINADOS

CONF. NBR 6009/80

PROPRIEDADES PARA PROJLTO

(continuação)

OBS: $I_{T}=$ MOMFNTO DE INERRCIA $\Lambda$ TORÇ $\bar{O}$

$\mathrm{C}_{\mathrm{w}}=$ CONSTANIII: DE IIMPLNAMINTTO

$\beta_{1}=$ PARÂMI:TRO DF TORÇĀO (INIF:ORME (VFR NRR 8800/86)

$\beta_{2}=$ PARÃMLTRO) DE TORÇĀO NĀO UNIIOORML: (VFR NBR 8800/86)

$E=$ MOEULOTIFELASTICIDADF. DO AÇO $=206.000 \mathrm{MPa}$

$\mathrm{G}=$ MÓDUI.O DE IEIASIICIDADE TRANSVERSAL DO AÇO $=0,385 . \mathrm{E}$

\begin{tabular}{|c|c|c|c|c|c|c|c|c|c|}
\hline \multirow{3}{*}{$\begin{array}{c}\text { DESIG. } \\
\text { IP }\end{array}$} & $P$ & \multicolumn{2}{|c|}{$\begin{array}{l}\text { MODUL. RESIST. } \\
\text { PLÄSTICOS } \\
\end{array}$} & \multirow{3}{*}{$\frac{\mathrm{I}_{\mathrm{T}}}{\mathrm{cm}^{4}}$} & \multirow{3}{*}{$\frac{C_{w}}{\mathrm{~cm}^{6}}$} & \multirow{3}{*}{$\frac{\mathrm{r}_{\mathrm{T}}}{\mathrm{cm}}$} & \multirow{3}{*}{$\frac{d / A_{f}}{c^{-1}}$} & \multirow{3}{*}{$\frac{\beta_{1} / W_{x}}{M P_{a}}$} & \multirow{3}{*}{$\begin{array}{r}\beta_{2} \\
-\end{array}$} \\
\hline & \multirow{2}{*}{$\mathrm{Kg} / \mathrm{m}$} & $\mathrm{Z}_{\mathrm{x}}$ & $\mathrm{z}_{\mathrm{y}}$ & & & & & & \\
\hline & & $\mathrm{cm}^{3}$ & $\mathrm{~cm}^{3}$ & & & & & & \\
\hline 80 & 6.0 & 23,2 & 5,75 & 0,70 & 118 & 1,22 & 3,34 & 46431 & 3914 \\
\hline 100 & 8,1 & 39.4 & 8.99 & 1,21 & 351 & 1,45 & 3,19 & 41451 & 4851 \\
\hline 120 & 10,4 & 60,8 & 13,4 & 1,74 & 890 & 1,69 & 2,98 & 36310 & 6285 \\
\hline 140 & 12,9 & 88.4 & 19,0 & 2,45 & 1980 & 1,93 & 2,78 & 32928 & 7600 \\
\hline 160 & 15,8 & 124 & 25,7 & 3,62 & 3960 & 2,15 & 2,64 & 31425 & 8286 \\
\hline 180 & 18,8 & 166 & 34,2 & 4,81 & 7430 & 2,39 & 2,47 & 29489 & 9421 \\
\hline 200 & 22,4 & 220 & 43,9 & 7,01 & 12990 & 2,59 & 2,33 & 29257 & 9555 \\
\hline 220 & 26,2 & 286 & 57,4 & 9,10 & 22670 & 2,87 & 2,17 & 27780 & 10453 \\
\hline 240 & 30,7 & 366 & 72,6 & 12,9 & 37390 & 3,11 & 2,04 & 27834 & 10294 \\
\hline 270 & 36,1 & 484 & 95,6 & 16,0 & 70580 & 3,51 & 1,96 & 25366 & 12409 \\
\hline 300 & 42,2 & 628 & 124 & 20,2 & 125900 & 3,90 & 1,87 & 23766 & 14286 \\
\hline 330 & 49,1 & 804 & 151 & 28,3 & 199100 & 4,13 & 1,79 & 23705 & 14381 \\
\hline 360 & 57,1 & 1020 & 189 & 37,5 & 313600 & 4,40 & 1,67 & 23193 & 14986 \\
\hline 400 & 66,3 & 1310 & 225 & 51,3 & 490000 & 4,63 & 1,64 & 22792 & 15769 \\
\hline 450 & 77.6 & 1700 & 273 & 67,2 & 791000 & 4,87 & 1,62 & 21813 & 17862 \\
\hline 500 & 90,7 & 2200 & 332 & 89,6 & 1249000 & 5,10 & 1,56 & 21211 & 19436 \\
\hline 550 & 106 & 2780 & 395 & 124 & 1884000 & 5,34 & 1,52 & 21214 & 19660 \\
\hline 600 & 122 & 3520 & 480 & 166 & 2846000 & 5,60 & 1,43 & 21049 & 20330 \\
\hline
\end{tabular}


ANEXO 5

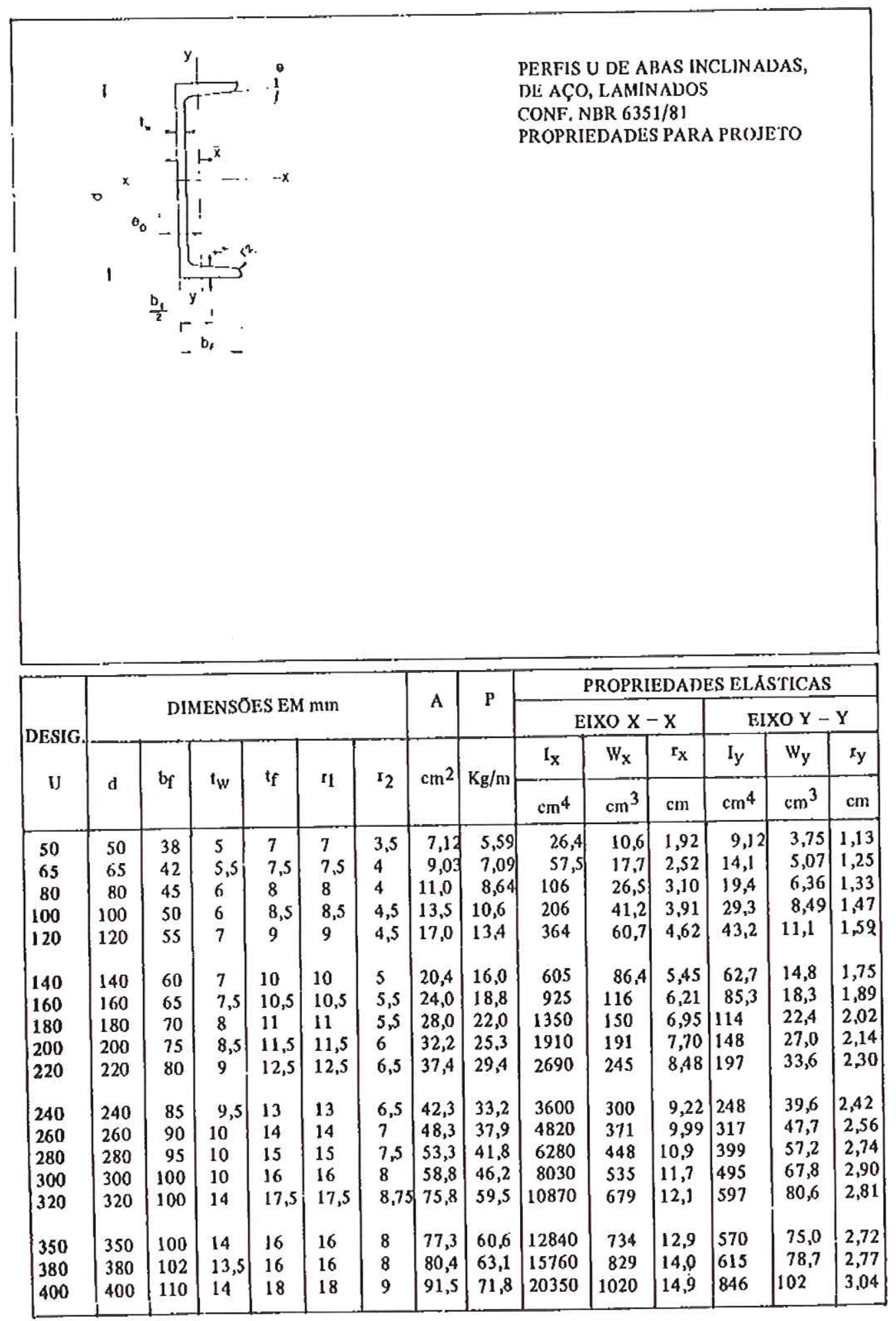




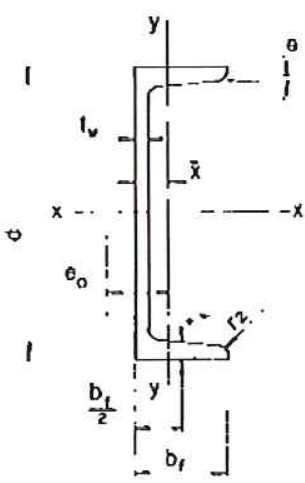

PERIIS U DF: ABAS INCLINADAS. DE: AÇO. LAMINADOS CONF. NBR 6351/81 PROPRIEDAULS PARA PROJETO (continuação)

$\theta=8 \%$ para $d \leqslant 300$ $\theta=5 \%$ para d $>300$

OBS: $\quad \mathbf{l}_{\mathrm{T}}=$ MOMLNTO DE INERCIA $\AA$ TORÇĀO

$\mathrm{C}_{\mathrm{W}}=$ CONSTANTL DF FMPENAMLNTO

$\beta_{1}=$ PARÃMETRO DE TORÇÀO UNIFORME (CONF. NBR 8800/86)

$\beta_{2}{ }^{\circ}=$ PARÃMETRO DE TORÇĀO NÃO UNIFORME (CONF. NBR 8800!86)

$\bar{x}$ - DISI'ANCIA DO CG A FACE EXTERNA DA ALMA

$\varepsilon_{0}$ - DISTANCIA DO CG AO CENTRO DE CIZALHAMENTO

\begin{tabular}{|c|c|c|c|c|c|c|c|c|c|c|}
\hline \multirow[t]{3}{*}{ DESIG. } & \multirow{3}{*}{$\mathrm{P}$} & \multicolumn{2}{|c|}{$\begin{array}{c}\text { MODULO RESIS- } \\
\text { SISTENTE PLAS- } \\
\text { TICO }\end{array}$} & \multirow{3}{*}{$\frac{\bar{x}}{c m}$} & \multirow{3}{*}{$\frac{\mathrm{e}_{\mathrm{o}}}{\mathrm{cm}}$} & \multirow{3}{*}{$\frac{\mathrm{I}_{\mathrm{r}}}{\mathrm{cm}^{4}}$} & \multirow{3}{*}{$\frac{C_{W}}{\mathrm{~cm}^{6}}$} & \multirow{3}{*}{$\frac{\mathrm{d} / \mathrm{A}_{\mathrm{f}}}{\mathrm{cns}-\mathrm{l}}$} & \multirow{3}{*}{$\frac{\beta_{1} / \mathrm{W}_{\mathrm{x}}}{\mathrm{MPa}}$} & \multirow{3}{*}{$\frac{\beta_{2}}{-}$} \\
\hline & & $z_{x}$ & $z_{y}$ & & & & & & & \\
\hline & & $\mathrm{cm}^{3}$ & $\mathrm{~cm}^{3}$ & & & & & & & \\
\hline 50 & 5,59 & - & - & 1,37 & 2,47 & 1,12 & 27,8 & 1,88 & 106977 & 753 \\
\hline 65 & 7,09 & 23,4 & 5,1 & 1,42 & 2,60 & 1,61 & 77,3 & 2,06 & 86503 & 1188 \\
\hline 80 & 8,64 & 31,8 & 16,6 & 1,45 & 2,67 & 2,16 & 168 & 2,22 & 73862 & 1692 \\
\hline 100 & 10,6 & 49,0 & 22,3 & 1,55 & 2,93 & 2,81 & 414 & 2,35 & 60030 & 2578 \\
\hline 120 & 13,4 & 72,6 & 30,2 & 1,60 & 3,03 & 4,15 & 900 & 2,42 & 55566 & 3235 \\
\hline 140 & 16,0 & 102,8 & 40,0 & 1,75 & 3,37 & 5,68 & 1800 & 2,33 & 50029 & 3890 \\
\hline 160 & 18,8 & 137,6 & 50.2 & 1,84 & 3,56 & 7,39 & 3260 & 2,34 & 46102 & 4652 \\
\hline 180 & 22,0 & 179,2 & 62.4 & 1,92 & 3,75 & 9,55 & 5570 & 2,34 & 43776 & 5367 \\
\hline 200 & 25,3 & 228 & 72,6 & 2,01 & 3,94 & 11,9 & 9070 & 2,32 & 41154 & 6162 \\
\hline 220 & 29,4 & 292 & 94,2 & 2,14 & 4,20 & 16,0 & 14600 & 2,20 & 40094 & 6450 \\
\hline 240 & 33,2 & 358 & 112 & 2,23 & 4,39 & 19, & 22100 & 2,17 & 38639 & 7091 \\
\hline 260 & 37,9 & 442 & 136 & 2,36 & 4,66 & 25,5 & 33300 & 2,06 & 37985 & 7346 \\
\hline 280 & 41,8 & 532 & 160 & 2,53 & 5,02 & 31,0 & 48500 & 2,87 & 36434 & 7338 \\
\hline 300 & 46,2 & 632 & 188 & 2,70 & 5,41 & 37,4 & 69100 & 1,87 & 35198 & 8127 \\
\hline 320 & $\$ 9,5$ & 826 & 215 & 2,60 & 4,82 & 66,7 & 96100 & 1,83 & 42051 & 6664 \\
\hline 350 & 60,6 & 918 & 205 & 2,4 & 4,4 & 61, & & 2,19 & 37628 & 9030 \\
\hline 380 & 63,1 & 1014 & 214 & 2,38 & 4,5 & 59,1 & 146000 & 2,33 & 33390 & 11552 \\
\hline 400 & 71,8 & 1240 & 279 & 2.65 & 5,11 & 81,6 & 221000 & 2,02 & 34017 & 10487 \\
\hline
\end{tabular}


ANEXO 6

Demonstração das Fórmulas dos Regimes de Membrana e de Flexão 


\section{Demonstração das Fórmulas dos Regimes de Membrana e de Flexão}

(considerando-se um carregamento hidrostático)

(Luciano Jorge de Andrade Junior. São Carlos, outubro de 1997.)

\section{1 ) Regime de Membrana $\left(\mathbf{N}_{\theta}\right.$ e $\left.\mathbf{N}_{\mathbf{z}}\right)$}

(ou seja, o fundo do reservatório é considerado livre para se deslocar)

\section{a) Carregamento hidrostático}

$$
\begin{array}{ll}
\mathrm{N}_{\theta}=-\mathrm{p}_{\mathrm{y}} \mathrm{r} & \mathrm{p}_{\mathrm{y}}=-\gamma \mathrm{z}+\gamma \mathrm{H} \therefore \mathrm{p}_{\mathrm{y}}=\gamma(\mathrm{H}-\mathrm{z}) \\
\mathrm{N}_{\theta}=-\gamma(\mathrm{H}-\mathrm{z}) \mathrm{r} & \mathrm{N}_{\mathrm{z}}=0 \\
\mathrm{w}(\mathrm{z})=\gamma(\mathrm{H}-\mathrm{z}) \cdot \frac{\mathrm{r}^{2}}{\mathrm{Ee}} &
\end{array}
$$

\section{2 ) Regime de Flexão $\left(\mathbf{N}_{\theta}, \mathbf{M}_{z}\right.$ e $\left.\mathbf{M}_{\theta}\right)$}

A expressão completa dos deslocamentos é dada por:

$$
\mathrm{w}(\mathrm{z})=\gamma(\mathrm{H}-\mathrm{z}) \cdot \frac{\mathrm{r}^{2}}{\mathrm{Ee}}+\exp (-\beta \mathrm{z})\left[\mathrm{C}_{3} \cos (\beta \mathrm{z})+\mathrm{C}_{4} \operatorname{sen}(\beta \mathrm{z})\right]
$$

\section{1 - Fundo não-engastado.}

Admitindo o fundo não-engastado, as condições de vinculação podem ser adotadas tais que:

$$
\begin{aligned}
& \text { w) } 0=0 \\
& \left.\frac{\mathrm{d}^{2} \mathrm{w}}{\mathrm{dz^{2 }}}\right)_{0}=0 \\
& \text { Condição (1): } \quad 0=\gamma \mathrm{H} \frac{\mathrm{r}^{2}}{\mathrm{Ee}}+\mathrm{C}_{3} \Rightarrow \mathrm{C}_{3}=-\gamma \mathrm{H} \frac{\mathrm{r}^{2}}{\mathrm{Ee}} \\
& \text { Condição (2) : } \quad \frac{\mathrm{dw}}{\mathrm{dz}}=-\gamma \frac{\mathrm{r}^{2}}{\mathrm{Ee}}-\beta \exp (-\beta \mathrm{z})\left[\mathrm{C}_{3} \cos (\beta \mathrm{z})+\mathrm{C}_{4} \operatorname{sen}(\beta \mathrm{z})\right] \\
& +\beta \exp (-\beta \mathrm{z})\left[-\beta \mathrm{C}_{3} \operatorname{sen}(\beta \mathrm{z})+\beta \mathrm{C}_{4} \cos (\beta \mathrm{z})\right]
\end{aligned}
$$




$$
\begin{aligned}
& \frac{\mathrm{d}^{2} \mathrm{w}}{\mathrm{dz}}=\beta^{2} \exp (-\beta \mathrm{z})\left[\mathrm{C}_{3} \cos (\beta \mathrm{z})+\mathrm{C}_{4} \operatorname{sen}(\beta \mathrm{z})\right]-\beta \exp (-\beta \mathrm{z})\left[-\beta \mathrm{C}_{3} \operatorname{sen}(\beta \mathrm{z})+\beta \mathrm{C}_{4} \cos (\beta \mathrm{z})\right] \\
& -\beta \exp (-\beta \mathrm{z})\left[-\beta \mathrm{C}_{3} \operatorname{sen}(\beta \mathrm{z})+\beta \mathrm{C}_{4} \cos (\beta \mathrm{z})\right]+\exp (-\beta \mathrm{z})\left[-\beta^{2} \mathrm{C}_{3} \cos (\beta \mathrm{z})-\beta^{2} \mathrm{C}_{4} \operatorname{sen}(\beta \mathrm{z})\right] \\
& \frac{\mathrm{d}^{2} \mathrm{w}}{\mathrm{dz}^{2}}=\beta^{2} \exp (-\beta \mathrm{z})\left[2 \mathrm{C}_{3} \operatorname{sen}(\beta \mathrm{z})-2 \mathrm{C}_{4} \cos (\beta \mathrm{z})\right] \\
& \left.\frac{\mathrm{d}^{2} \mathrm{w}}{\mathrm{dz}^{2}}\right)_{0}=0 \Rightarrow 0=\mathrm{C}_{3} \operatorname{sen}(\beta \mathrm{z})-\mathrm{C}_{4} \cos (\beta \mathrm{z}) \because \operatorname{sen}(\beta \mathrm{z})_{\mathrm{z}=0}=0 \therefore \mathrm{C}_{4}=0 \\
& \frac{\mathrm{d}^{3} \mathrm{w}}{\mathrm{dz}}=-2 \beta^{3} \exp (-\beta \mathrm{z})\left[\mathrm{C}_{3} \operatorname{sen}(\beta \mathrm{z})-\mathrm{C}_{4} \cos (\beta \mathrm{z})\right]+2 \beta^{2} \exp (-\beta \mathrm{z})\left[\beta \mathrm{C}_{3} \cos (\beta \mathrm{z})+\beta \mathrm{C}_{4} \operatorname{sen}(\beta \mathrm{z})\right] \\
& \frac{\mathrm{d}^{3} \mathrm{w}}{\mathrm{dz}}=2 \beta^{3} \exp (-\beta \mathrm{z})\left\{\mathrm{C}_{3}[\operatorname{sen}(\beta \mathrm{z})+\cos (\beta \mathrm{z})]+\mathrm{C}_{4}[\operatorname{sen}(\beta \mathrm{z})-\cos (\beta \mathrm{z})]\right\}
\end{aligned}
$$

Pelos cálculos efetuados e substituindo-se os valores de $\mathrm{C}_{3}$ e $\mathrm{C}_{4}$, a expressão dos deslocamentos resulta:

$$
\begin{aligned}
& \mathrm{w}(\mathrm{z})=\gamma \frac{\mathrm{r}^{2}}{\mathrm{Ee}}[(\mathrm{H}-\mathrm{z})-\mathrm{H} \cos (\beta \mathrm{z}) \exp (-\beta \mathrm{z})] \\
& \beta^{4}=\frac{3\left(1-v^{2}\right)}{\mathrm{r}^{2} \mathrm{e}^{2}} \\
& \mathrm{D}=\frac{\mathrm{Ee}^{3}}{12\left(1-v^{2}\right)}
\end{aligned}
$$

A partir de w(z), os esforços $\mathrm{N}_{\theta}, \mathrm{M}_{\mathrm{Z}}, \mathrm{M}_{\theta}$ e $\mathrm{Q}_{\mathrm{Z}}$ podem ser calculados:

$$
\begin{aligned}
& \mathrm{N}_{\theta}=-\frac{\mathrm{Ee}}{\mathrm{r}} \mathrm{w} \therefore \mathrm{N}_{\theta}=-\gamma[\mathrm{H}-\mathrm{z}-\mathrm{H} \exp (-\beta \mathrm{z}) \cos (\beta \mathrm{z})] \\
& \mathrm{M}_{\mathrm{z}}(\mathrm{z})=-\mathrm{D}\left[\frac{\mathrm{d}^{2} \mathrm{w}}{\mathrm{dz}^{2}}\right] \therefore \mathrm{M}_{\mathrm{z}}(\mathrm{z})=\frac{\gamma \mathrm{Hr}^{2} \mathrm{e}^{2} \beta^{2}}{6\left(1-v^{2}\right)} \exp (-\beta \mathrm{z}) \operatorname{sen}(\beta \mathrm{z}) \\
& \mathrm{M}_{\theta}=\mathrm{LM}_{\mathrm{z}} \\
& \mathrm{Q}_{\mathrm{z}}=-\mathrm{D} \frac{\mathrm{d}^{3} \mathrm{w}}{\mathrm{dz}^{3}} \therefore \mathrm{Q}_{\mathrm{z}}=\frac{\mathrm{e}^{2} \beta^{3} \exp (-\beta \mathrm{z}) \gamma \mathrm{Hr}^{2}}{6\left(1-v^{2}\right)}[\operatorname{sen}(\beta \mathrm{z})+\cos (\beta \mathrm{z})]
\end{aligned}
$$




\section{2 - Fundo engastado}

Admitindo o fundo engastado, as condições de contorno são:
w) $0=0$

$$
\left.\frac{\mathrm{dw}}{\mathrm{dz}}\right)_{0}=0
$$

Condição (1): $\quad 0=\gamma \frac{\mathrm{Hr}^{2}}{\mathrm{Ee}}+\mathrm{C}_{3} \therefore \mathrm{C}_{3}=-\gamma \frac{\mathrm{Hr}^{2}}{\mathrm{Ee}}$

$\begin{aligned} \text { Condição (2): } & \frac{\mathrm{dw}}{\mathrm{dz}}=-\gamma \frac{\mathrm{r}^{2}}{\mathrm{Ee}}-\beta \exp (-\beta \mathrm{z})\left[\mathrm{C}_{3} \cos (\beta \mathrm{z})+\mathrm{C}_{4} \operatorname{sen}(\beta \mathrm{z})\right] \\ & +\exp (-\beta \mathrm{z})\left[-\beta \mathrm{C}_{3} \operatorname{sen}(\beta \mathrm{z})+\beta \mathrm{C}_{4} \cos (\beta \mathrm{z})\right]\end{aligned}$

$$
\left.\frac{\mathrm{dw}}{\mathrm{dz}}\right)_{0}=0 \Rightarrow 0=-\gamma \frac{\mathrm{r}^{2}}{\mathrm{Ee}}-\beta \mathrm{C}_{3}+\beta \mathrm{C}_{4} \Rightarrow \mathrm{C}_{4}=\gamma \frac{\mathrm{r}^{2}}{\mathrm{Ee}}+\mathrm{C}_{3}
$$

$\mathrm{C}_{4}=\gamma \frac{\mathrm{r}^{2}}{\mathrm{Ee}}\left(\frac{1}{\beta}-\mathrm{H}\right)$

Substituindo-se os valores de $\mathrm{C}_{3}$ e $\mathrm{C}_{4}$ na equação dos deslocamentos, w(z) resulta:

$$
\mathrm{w}(\mathrm{z})=\gamma(\mathrm{H}-\mathrm{z}) \frac{\mathrm{r}^{2}}{\mathrm{Ee}}+\exp (-\beta \mathrm{z})\left[-\gamma \mathrm{H} \frac{\mathrm{r}^{2}}{\mathrm{Ee}} \cos (\beta \mathrm{z})+\gamma \frac{\mathrm{r}^{2}}{\mathrm{Ee}}\left(\frac{1}{\beta}-\mathrm{H}\right) \operatorname{sen}(\beta \mathrm{z})\right]
$$

Os esforços são dados então por:

$$
\begin{aligned}
& \mathrm{N}_{\theta}=-\frac{\mathrm{Ee}}{\mathrm{r}} \mathrm{w} \therefore \\
& \mathrm{N}_{\theta}(\mathrm{z})=\gamma \cdot\left\{\mathrm{H}-\mathrm{z}+\exp (-\beta \mathrm{z})\left[-\mathrm{H} \cos (\beta \mathrm{z})+\left(\frac{1}{\beta}-\mathrm{H}\right) \operatorname{sen}(\beta \mathrm{z})\right]\right\}
\end{aligned}
$$




$$
\begin{aligned}
& M_{z}=-D\left[\frac{d^{2} w}{d z^{2}}\right] \therefore \\
& M_{z}=-\gamma \frac{e^{2} \beta^{2} r^{2}}{6\left(1-v^{2}\right)} \exp (-\beta z)\left[-H \operatorname{sen}(\beta z)-\left(\frac{1}{\beta}-H\right) \cos (\beta z)\right] \\
& M_{\theta}=M_{z} \\
& Q_{z}=-D\left[\frac{d^{3} w}{d z^{3}}\right] \therefore \\
& Q_{z}=-\gamma \frac{e^{2} \beta^{3} r^{2}}{6\left(1-v^{2}\right)} \exp (-\beta z)\left\{-2 H \operatorname{sen}(\beta z)+\frac{1}{\beta}[\operatorname{sen}(\beta z)-\cos (\beta z)]\right\}
\end{aligned}
$$

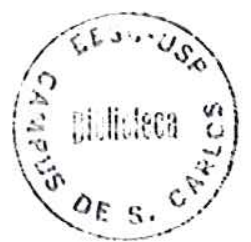

